GA-A21647

UC-712

\title{
INERTIAL CONFINEMENT FUSION TARGET COMPONENT FABRICATION AND TECHNOLOGY DEVELOPMENT SUPPORT
}

\author{
ANNUAL REPORT TO THE \\ U.S. DEPARTMENT OF ENERGY
}

OCTOBER 1, 1992 THROUGH SEPTEMBER 30, 1993

\author{
by \\ PROJECT STAFF \\ David Steinman, Editor
}

\author{
Work supported by \\ U.S. Department of Energy \\ under Contract No. DE-AC03-91SF18601
}

GENERAL ATOMICS PROJECT 3896

DATE PUBLISHED: MARCH 1994

MASTER

$n^{p}$ 


\title{
THE 1993 TARGET FABRICATION AND TARGET TECHNOLOGY DEVELOPMENT TEAM
}

\author{
GENERAL ATOMICS \\ SAN DIEGO, CALIFORNIA
}

Neil Alexander, Jan Ankney, Wes Baugh, Chuck Beal, Don Beighley, Gary Bentley, Bob Bourque, Lloyd Brown, Sharon Considine, Bob Fagaly, Jane Gibson, Kett Gifford, lleese Glatter-Schneir, Steve Grant, Martin Hoppe, Dave Husband, Jim Kaae, Jim Lindgren, Tu Luong, Roy Mangano, Barry McQuillan, Wayne Miller, Paul Parks, Joe Pontelandolfo, John Ruppe, Chuck Schneidmuller, Ken Schultz, Clyde Shearer, John Simpson, Naomi Skolmutch, Rich Stephens, Dave Steinman, Don Wall.

\section{W. J. SCHAFER ASSOCIATES \\ LIVERMORE, CALIFORNIA}

Tom Alberts, Bob Bieri, Don Bittner, Frank Carey, Amy Carter, Andrea Denton, Scott Faulk, Barbara Lane, David Diffenderfer, Illges Faron, Chuck Hendricks, Cynthia Keller, Michael McClellan, Michael Monsler, Brian Motta, Craig Rivers, Jim Sater, Diana SchroenCarey, Keith Shillito.

SOANE TECHNOLOGIES, INC. HAYWARD, CALIFORNIA

Emil Asadulla, Sue Bernard, Lisa Chuong, Eben Lilley, Don Nelson, David Soane, Zoya Soane. 


\section{ABSTRACT}

On December 30, 1990, the U.S. Department of Energy entered into a contract with General Atomics (GA) to be the Inertial Confinement Fusion (ICF) Target Component Fabrication and Technology Development Support contractor. This report documents the technical activities which took place under this contract during the period of October 1, 1992 through September 30, 1993. During this period, GA was assigned 18 tasks in support of the Inertial Confinement Fusion program and its laboratories. These tasks included "Capabilities Activation" and "Capabilities Demonstration" to enable us to begin production of glass and composite polymer capsules. Capsule delivery tasks included "Small Glass Shell Deliveries" and "Composite Polymer Capsules" for Lawrence Livermore National Laboratory (LLNL) and Los Alamos National Laboratory (LANL).

We also were asked to provide direct "Onsite Support" at LLNL and LANL. We continued planning for the transfer of "Micromachining Equipment from Rocky Flats" and established "Target Component Micromachining and Electroplating Facilities" at GA. We fabricated over 1100 films and filters of 11 types for Sandia National Laboratory (SNL) and provided full-time onsite engineering support for target fabrication and characterization.

We initiated development of methods to make targets for the Naval Research Laboratory (NRL). We investigated spherical interferometry, built an automated capsule sorter, and developed an apparatus for calorimetric measurement of fuel fill for LLNL. We assisted LANL in the "Characterization of Opaque b-Layered Targets". We developed deuterated and UV-opaque polymers for use by the University of Rochester's Laboratory for Laser Energetics (UR/LLE) and devised a triple-orifice droplet generator to demonstrate the controlled-mass nature of the microencapsulation process.

We began assembly of equipment for a cryogenic target handling system for UR/LLE that will fill, transport, layer, and characterize targets filled with cryogenic deuterium (DD) or deuterium-tritium (DT) fuel, and insert these cryogenic targets in the OMEGA Upgrade target chamber for laser implosion experiments.

This report summarizes and documents the technical progress made on these tasks. 


$$
\text { = }
$$




\section{TABLE OF CONTENTS}

1. Target Fabrication Program Overview ............................................................... 1-1

1.1. INTRODUCTION ............................................................................... 1-1

1.2. FY 93 TASKS SUMMARY.............................................................. 1-1

1.2.1. GA01 Capabilities Activation.................................................... 1-1

1.2.2. GA02 Capabilities Demonstration.............................................. 1-3

1.2.3. LL01 Onsite Support at LLNL ................................................. 1-4

1.2.4. LL02 Micromachining Equipment from Rocky Flats ................... 1-4

1.2.5. LL03 Small Glass Shell Deliveries ............................................. 1-4

1.2.6. LL04 Composite Polymer Capsules ............................................. 1-5

1.2.7. LL05 Characterization Development ........................................ 1-7

1.2.8. LL06 Micromachined Target Component Facility ........................ 1-8

1.2.9. LL07 Target Component Electroplating Facility ......................... 1-9

1.2.10. LA01 Composite Polymer Capsules ................................................ 1-9

1.2.11. LA02 Onsite Support for LANL................................................. 1-9

1.2.12. LA03 Characterization of Opaque Beta-Layered Targets ............ 1-11

1.2.13. LA04 Microencapsulated Shells ................................................. 1-11

1.2.14. LA06 Small glass Shell Deliveries ............................................ 1-13

1.2.15. NR01 Naval Research Laboratory (NRL) Target Development... 1-13

1.2.16. SL01 Fabrication of Foils and Filters....................................... 1-15

1.2.17. UR01 Omega Upgrade Cryogenic Target System....................... 1-15

1.2.18. UR02 LLE Materials Development ........................................... 1-16

2. CAPABILITIES DEVELOPMENT ………..................................................... 2-1

2.1. SHELL FABRICATION........................................................................ 2-1

2.1.1. Polystyrene Shell Tower.............................................................. 2-1

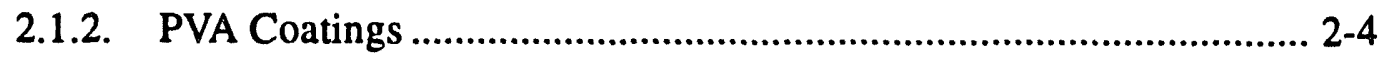

2.1.3. Frit Precursor ....................................................................... 2-5

2.1.4. Glass Shell Tower................................................................. 2-6 


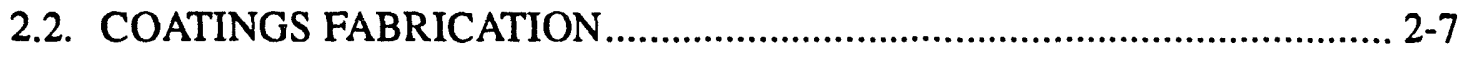

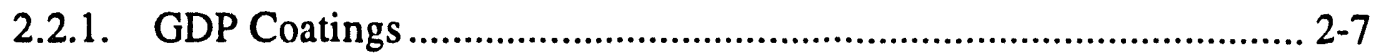

2.2.2. Parylene Coatings .................................................................. 2-12

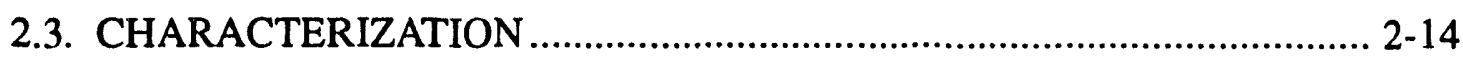

2.3.1. Optical Techniques - PVA Cull ................................................. 2-14

2.3.2. X-Ray Radiography - Image Analysis .......................................... 2-16

2.3.3. Index of Refraction Measurements - In Situ ................................ 2-20

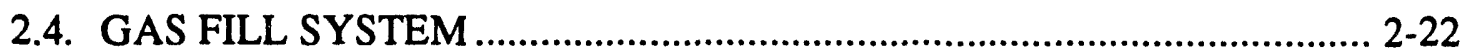

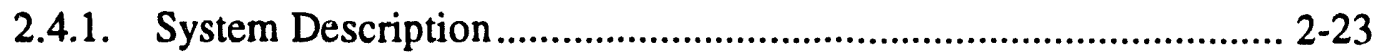

2.4.2. Glass Shell Fill Test ................................................................ 2-25

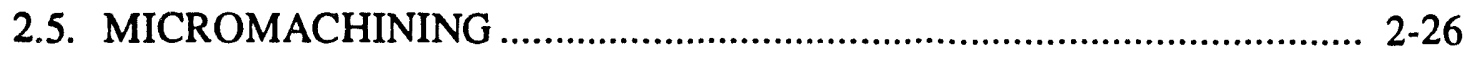

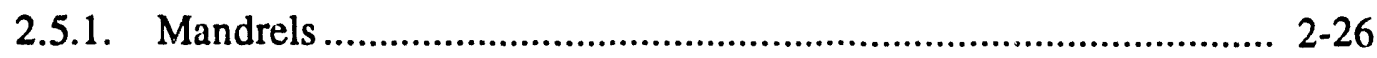

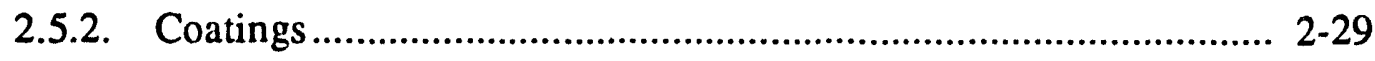

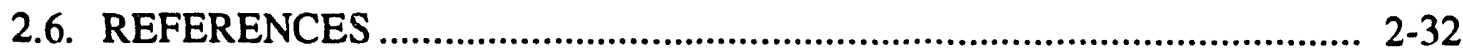

3. LABORATORY RESEARCH SUPPORT ................................................... 3-1

3.11. CRYOGENIC TRITIUM RESEARCH AT LANL ……........................... 3-1

3.1.1. Introduction ............................................................................ 3-1

3.1.2. Experimental Set-Up......................................................... 3-2

3.1.3. DT Solid Layer Experiments ................................................... 3-5

3.1.4. Conclusions................................................................................... 3-11

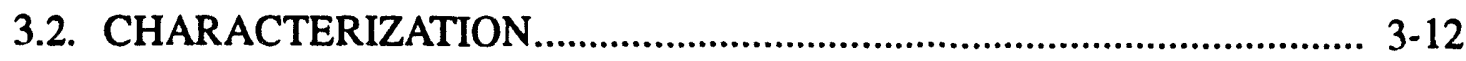

3.2.1. Resonant Ultrasound Spectroscopy ........................................... 3-13

3.2.2. Spatially Resolved Nuclear Magnetic Resonance ......................... 3-16

3.3. CHARACTERIZATION DEVELOPMENT AT LLNL ............................. 3-20

3.3.1. Calorimetric Measurement of Fuel Fill ....................................... 3-21

3.3.2. Spherical Interferometry .......................................................... 3-23

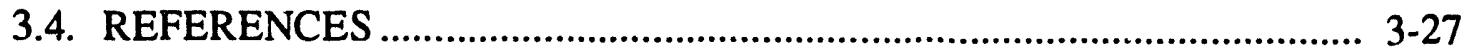

4. MICROENCAPSULATION PROCESS DEVELOPMENT................................. 4-1

4.1. INTRODUCTION ................................................................................ 4-1

4.2. POLYMER SCIENCE .......................................................................... 4-2 


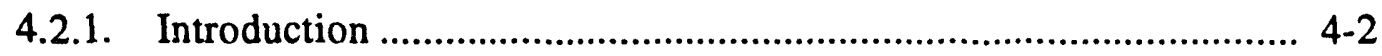

4.2.2. Mass Transfer Modeling ............................................................ 4-2

4.2.3. Polymer Solution Thermodynamics............................................... 4-4

4.3. STATISTICAL PROCESS ANALYSIS................................................... 4-5

4.3.1. Design........................................................................................ 4-6

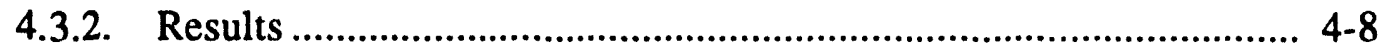

4.3.3. Conclusion .............................................................................. 4-13

4.4. CONTROLLED-MASS SYSTEM DEVELOPMENT ................................ 4-13

4.4.1. Triple-Orifice Droplet Generator ................................................ 4-13

4.4.2. Solidification Column Design..................................................... 4-14

4.4.3. Characterization ........................................................................ 4-15

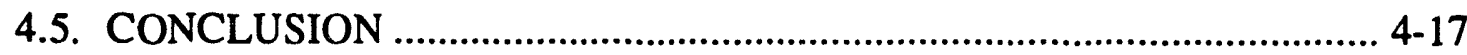

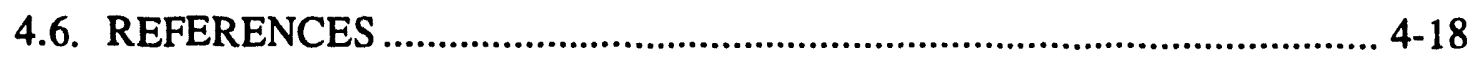

5. NRL TARGET DEVELOPMENT ……............................................................ 5-1

5.1. NRL Target Fabrication Goals................................................................. 5-1

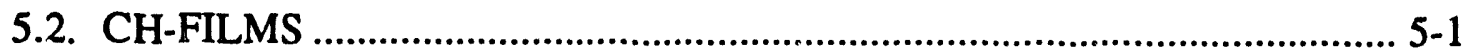

5.2.1. CH-Film Production ................................................................... 5-2

5.2.2. CH-Films Target Mount Design and Production............................. 5-5

5.2.3. Characterization of $\mathrm{CH}$-Films ..................................................... 5-6

5.2.4. Assembly of Prototype Nike CH-Film Targets ............................. 5-11

5.3. DESIGN AND IMPLEMENTATION OF APPARATUS FOR CRYOGENIC LAYER TARGETs....................................................... 5-14

6. FABRICATION OF FILMS AND FILTERS FOR SNL …................................. 6-1

6.1. FABRICATION OF FOILS AND FILTERS................................................ 6-1

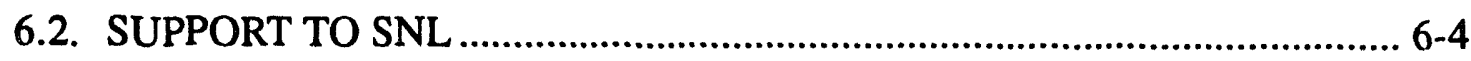

7. OMEGA UPGRADE CRYOGENIC TARGET DELIVERY SYSTEM ................. 7-1

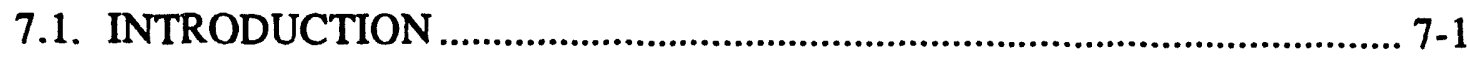

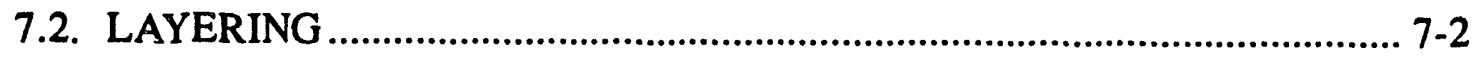

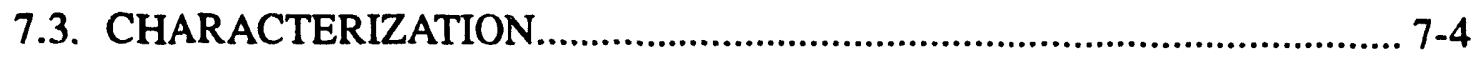




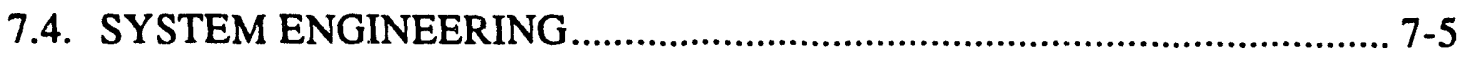

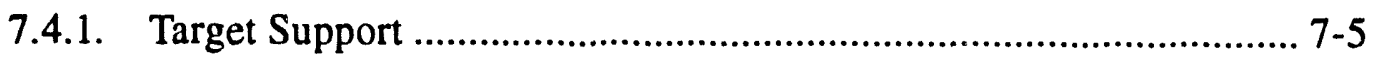

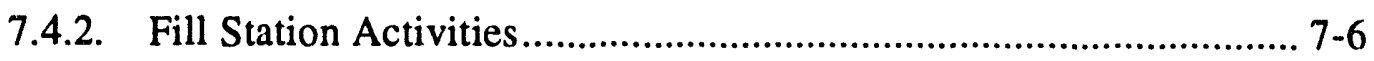

7.4.3. Cryogenic Transport and Storage................................................... 7-7

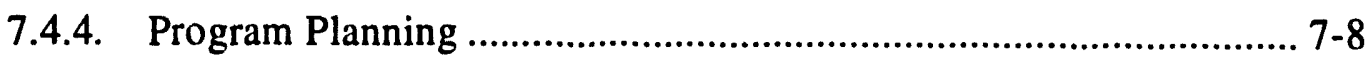

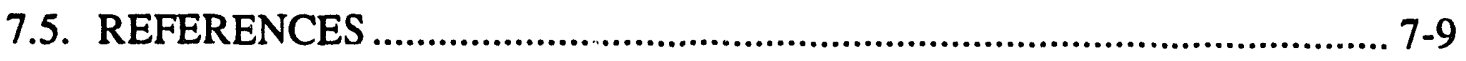

8. LLE MATERIALS DEVELOPMENT ............................................................ 8-1

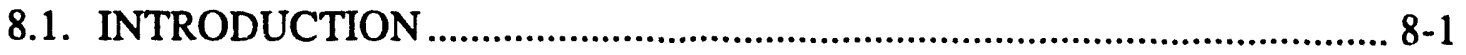

8.2. POLY[PERDEUTERO(TRIMETHYLSILYL)STYRENE] .......................... 8-1

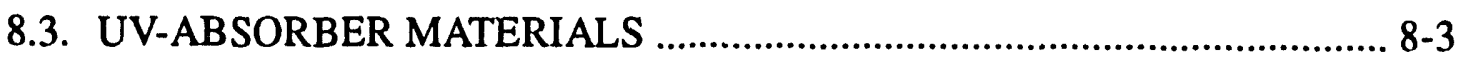

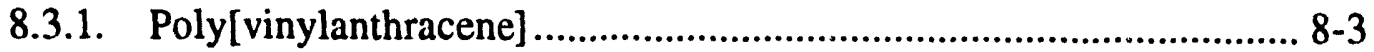

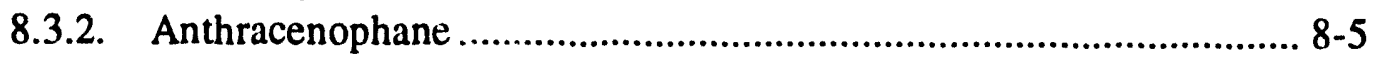

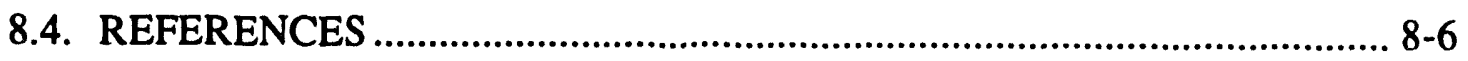

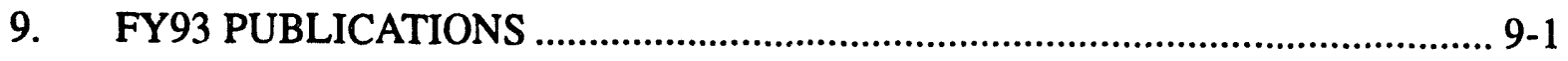

\section{LIST OF FIGURES}

2-1. The piezoelectric droplet generator ........................................................ 2-2

2-2. Volume displacement of the piezoelectric droplet generator ............................ 2-3

2-3. Temperature uniformity of PVA tower as a function of fan configuration ....... 2-5

2-4. Top view of modified PVA tower oven .................................................... 2-6

2-5. SEM Micrograph of the surface of a capsule coated using the sccond

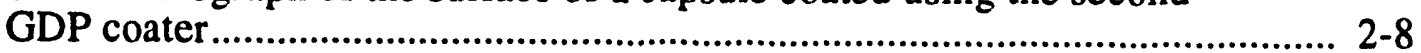

2-6. $\mathrm{H}_{\alpha}$ line emission of GDP coater over 11.5 hours ........................................ 2-9

2-7. SEM micrograph of Germanium-doped $\mathrm{CH}$ coating deposited on a silicon wafer.

2-8. Schematic of the Parylene Coating System ……...................................... 2-12

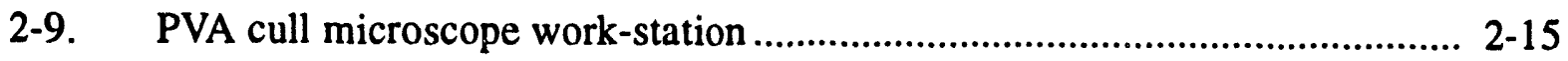

2-10. Image of the same shell under (a) diffuse, (b) collimated, and

(c) dark field illumination 
2-11. Image of shells using white-light interference......................................... 2-17

2-12. PVA cull statistics worksheet .............................................................. 2-18

2-13. X-radiograph image of composite shell using $5 \mathrm{keV}$ radiation...................... 2-19

2-14. Radial film density profile for a shell compared to two simulated curves...... 2-21

2-15. Side view of apparatus used for determining index of refraction of sample material

2-16. White light interference micrographs of objects in index matching solution.

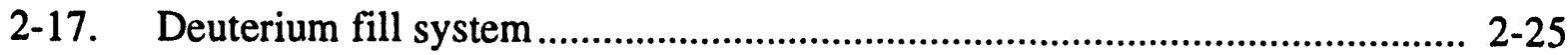

2-18. Drawing of the test mandrel for micromachining capability validation ......... 2-27

2-19. A scanning electron micrograph of a micromachined test mandrel............... 2-28

2-20. A profilometer trace of the barrel surface of a test mandrel......................... 2-29

2-21. A cross-sectional drawing of the specimen rotator....................................... 2-31

3-1. Schematic of high resolution optical DT layering experiment at LANL .......... 3-3

3-2. Graph (a) of image motion for random and synchronized image acquisition, Graph (b) showing scatter in image focus.

3-3. Total cumulative (RMS) scatter in surface roughness for random and synchronized image acquisition

3-4. CCD image of $125 \mu \mathrm{m} \mathrm{DT}$ solid layer inside a $2 \mathrm{~mm}$ diameter copper cell ..... 3-6

3-5. FFT power spectra for $125 \mu \mathrm{m}$ DT solid layer and empty copper cell .............. 3-7

3-6. Comparison of DT surface roughness for 73 and $125 \mu \mathrm{m}$ DT solid layers....... 3-8

3-7. CCD image of $139 \mu \mathrm{m}$ DT solid layer inside $2 \mathrm{~mm}$ diameter copper cell...... 3-10

3-8. Solid surface power spectra for $139 \mu \mathrm{m}$ DT solid layer and empty copper cell

3-9. Time evolution of $139 \mu \mathrm{m}$ DT solid layer surface finish, at three temperatures

3-10. Surface finish of the $139 \mu \mathrm{m}$ DT solid layer at three temperatures 
3-11. RUS pinducers arranged around the target in a tetrahedral configuration..... 3-14

3-12. Analysis of RUS spectra from two separate receivers on same sphere........... 3-15

3-13. Experimental concepts for spatially resolved NMR of beta-layered DT ........ 3-17

3-14. NMR probe and cryostat are shown removed from the magnet .................... 3-18

3-15. Proton 1-D projection image of water contained in five horizontally oriented tubes using the gradient Hahn echo

3-16. Proton 1-D projection image of solidified- $\mathrm{H}_{2}$ and gaseous $-\mathrm{H}_{2}$ above the solid using the gradient Free Induction Decay (FID)

3-17. Measured heat capacity of shell and calorimeter.......................................... 3-23

3-18. Schematic of the interferometric setup.................................................. 3-24

3-19. Power spectral density from the AFM measurements .................................. 3-26

3-20. Power spectral density from the interferometer measurements ..................... 3-27

4-1. Percentage of initial solvent remaining versus time ..................................... 4-4

4-2. Solvent concentration at outer edge of shell as a function of outer radius ....... 4-5

4-3. Modeling results and how it is incorporated with phase equilibrium data........ 4-6

4-4. Data from Thermal Optical Analysis experiments comparing the effect of molecular weight on phase behavior

4-5. Graph showing the three axis solvent system............................................. 4-9

4-6. Graphs indicating the results for mean diameter and vacuole concentration

4-7. Graphs of mean diameter and vacuole density using solubility parameter ratios

4-8. Design of the triple-orifice droplet generator ............................................. 4-14

4-9. Arrangement of the controlled-mass system which improves output ............. 4-16

5-1. Slide holding fixture for producing thin $\mathrm{CH}$-films ..................................... 5-3

5-2. Schematic drawing of the $\mathrm{CH}-$ film target mount............................................ 5-6 
5-3. Photograph of an assembled $\mathrm{CH}$-film target ........................................... 5-7

5-4. Photograph of an interference pattern of a film which has a thickness variation of less than $10 \mathrm{~nm}$ over a $2 \mathrm{~mm}$ region in the center of the film ....... 5-8

5-5. Example of surface morphology obtained from the PTM .............................. 5-9

5-6. A $6 \mathrm{~nm}$ deep scratch on the surface of a $\mathrm{CH}$-film surface ........................... 5-10

5-7. PTM image of silicon chip roughness standard in which a series of grooves have been etched

5-8. Hot wire arrangement for cutting $\mathrm{CH}$-films ........................................... 5-12

5-9. Completed CH-film target in its storage container ...................................... 5-13

6-1. Examples of frames used to support foils requested by SNL ......................... 6-2

6-2. Cathodic sputtering device used to deposit gold on Mylar............................... 6-3

6-3. Different types of foils and filters fabricated for SNL in FY93....................... 6-5

7-1. Liquid layering flow diagram ("decision tree").............................................. 7-3

7-2. The tripod mounting technique for supporting ICF targets ...............................

7-3. The keyhole manipulator used for picking up targets...................................... 7-9

8-1. UV-visable spectrum of poly(vinylanthracene) …........................................ 8-4

\section{LIST OF TABLES}

2-1. GDP Production Coating Run Statistics.................................................... 2-7

2-2. Capabilities of the Parylene Coating System............................................. 2-13

4-1. Predicted and Measured Wall Thickness for Several ControlledMass Runs

6-1. Foils and Filters Fabricated at General Atomics in FY93 …........................... 6-4

8-1. UV-Absorption of Poly(vinylanthracene) ................................................. 8-5 
SECTION 1

TARGET FABRICATION PROGRAM OVERVIEW 


\section{TARGET FABRICATION PROGRAM OVERVIEW}

On December 30, 1990, the U.S. Department of Energy entered into a contract with General Atomics (GA) to be the Inertial Confinement Fusion (ICF) Target Component Fabrication and Technology Development Support contractor. This report documents the technical activities of the period October 1, 1992 through September 30, 1993. GA was assisted by W.J. Schafer Associates (WJSA) and Soane Technologies, Inc. (STI) and we have carried out the ICF Target Fabrication tasks as a fully integrated team effort.

\subsection{INTRODUCTION}

In FY93, the GA/WJSA/STI team achieved production capability in almost all areas of ICF target fabrication.

\subsection{FY93 TASKS SUMMARY}

During FY93, the GA/WJSA/STI team was assigned 18 formal tasks.

\subsubsection{GA01 CAPABILITIES FACILITIES}

The Capability Activation task was completed in the key areas of coatings and characterization so that production and delivery of ICF capsules and components to the national ICF Laboratories could commence.

\subsubsection{COATINGS}

Glow Discharge Polymerization (GDP) Coatings. The GDP coatings task was separated into two subtasks: validation of the second GDP coater assembled at General Atomics and development of diagnostics to aid in production runs of the coaters. In the first subtask, we produced good coatings in the second GDP coater by April, and subsequently demonstrated that this coater is capable of depositing doped coatings by depositing a germanium-doped GDP coating. 
The two diagnostic techniques that we developed and are utilizing in coating production runs are: (1) monitoring of the $\mathrm{H}_{\alpha}$ line emission intensity to record the plasma behavior, and (2) measuring diameters of capsules by optical microscopy while they are still in the coater to rapidly determine when to stop coating runs without exposing the capsules to air.

Parylene Coatings. This task consisted of upgrading the parylene coating system to give it ICF capsule coating capability. In FY92, the basic coater was assembled and demonstrated. In FY93, better temperature control was added to the coater, contamination from quartz wool in the pyrolyzer region was eliminated, and a baffle system was installed between the pyrolyzer and the coating regions to control particulates.

We installed a bounce pan (provided by the University of Rochester) to give us the capability of coating capsules. To keep the capsules from sticking in the bounce pan, a glow-discharge system was introduced into the coater. Finally, we built an in-situ thickness monitor into the coater.

\subsubsection{CHARACTERIZATION}

The objectives of this portion of the Capabilities Development Task were to improve the throughput of existing microscope and profilometer techniques, and to add a suite of new techniques including: $x$-radiography, $x$-ray fluorescence, refractometry, and gas pressure and composition determination.

Improving throughput involved many aspects and continued throughout the year. We added new equipment, trained personnel, and developed procedures. A profilometer and a scanning electron microscope were set up at WJSA and have been in use characterizing parylene coatings and $x$-ray pinhole arrays, respectively. At the GA site, two zoom microscopes were set up to cull shell batches, a second Jenavert interference microscope was added to characterize deliverable shells, and two Nikon microscopes were purchased, but have not yet been delivered. The two Nikons contain specialized optics; one microscope will be used for characterizing micromachined metal surfaces and the other for doing interference microscopy on large shells (up to $4 \mathrm{~mm}$ diameter). In addition, technicians at both WJSA and GA were trained in optical characterization techniques, and WJSA personnel assisted STI in setting up and properly using the microscopes STI bought with its own funds. Finally, characterization techniques were developed to improve the accuracy and speed of shell characterization. 
The x-radiography system was installed, is state licensed, and is operational. A dark room was set up to develop the film, and computer software was developed to analyze radiograph images. The paperwork for purchasing the $\mathrm{x}$-ray fluorescence system was reviewed by the Department of Energy (DOE) and approved in November 1993. The instrument was ordered and will be delivered by early 1994 . The refractometer system is operational. It has been used to verify our index matching fluids and to directly measure the refractive index of films of plastic used for shells.

The gas pressure and composition determination system has been designed and the major components (vacuum and gas chromatography systems) have been put out to bid and will be purchased on capital. A few parts remain to be machined; assembly and installation should be finished in FY94.

\subsubsection{GAO2 CAPABILITIES DEMONSTRATION}

Under task GA02 we completed work initiated in FY92 involving the tower furnace for glass shell production, a gas permeation fill station for glass shells, characterization capabilities, and polymer shell preparation by microencapsulation.

Glass Shells. We completed assembly of the glass shell tower. A quartz process tube was installed with a liner to capture the alkali vapors (mainly sodium) which contributed to premature tube failure. Most of the control system was replaced or upgraded. We tested the system and it performed well at $1200^{\circ} \mathrm{C}$.

Gas Fill Statinn. We completed the assembly of a station that can fill glass shells with up to 400 atmospheres of deuterium at $360^{\circ} \mathrm{C}$ (equivalent to 200 atmospheres at room temperature). We did a test fill on glass shells and determined that the shells had filled with deuterium as expected.

Characterization Capabilities. We submitted shells we had characterized to Lawrence Livermore National Laboratory (LLNL) for their evaluation of our measurements. LLNL validated our measurements.

Microencapsulation. We submitted poiymer shells we had prepared by microencapsulation to the University of Rochester's Laboratory for Laser Energetics (UR/LLE) for their evaluation of shell quality. UR/LLE found our shells had state-of-theart quality. 


\subsubsection{LLO1 ONSITE SUPPORT AT LLNL}

Our onsite team at LLNL emphasized activities in micromachining of target components, assembling target components into complete targets, and characterizing target components to assure their quality for use in Nova ICF experiments. In micromachining, Craig Rivers made hohlraum components, Thompson scattering targets, "gas box" experimental targets, and many auxiliary target components such as shields, back-lighters, and shaped wires. John Ruppe assembled nearly 100 classified hohlraums, and an equal number of unclassified targets of many designs. In characterization, Andrea Denton determined the key properties of targets and target assemblies by a variety of techniques including: radiography with image analysis, interferometry, optical microscopy, and Energy Dispersive X-ray Spectroscopy (EDXS).

\subsubsection{LLO2 MICROMACHINING EQUIPMENT FROM ROCKY FLATS}

We were tasked by DOE, in June 1993, to transfer micromachining equipment to California from the DOE Rocky Flats Plant in Colorado. We prepared site locations at GA and WJSA with the utilities necessary to accept and install the DOE micromachining equipment. The facility modifications required to install the micromachining equipment were completed at WJSA and a compressor system (for the air-bearing lathes) and thermal environmental enclosure (to maintain constant temperature) were procured and received. The actual installations will take place in early FY94. The Phase I disassembly and packaging of the micromachining equipment at Rocky Flats did not begin until late September 1993 due to a delay by EG\&G at Rocky Flats. We expect to continue with the removal of the Phase I micromachining equipment from Rocky Flats with completion in early FY94. Phase II equipment removal has not been planned or scheduled at this time.

\subsubsection{LLO3 SMALL GLASS SHELL DELIVERIES}

The goal of this task was to manufacture and deliver approximately 100 high quality spherical glass shells to meet LLNL's experimental requirements. We needed to complete the shakedown of the glass shell tower and develop glass frit production capability. In the interim, while awaiting completion of the production facilities, we were to sort through available stock to find suitable shells for delivery. 
Glass Shell Process Development. The spray dryer was successfully activated and produced monosized frit for glas? shell production. Unfortunately, the design of the spray dryer was such that the frit became contaminated with metal particles from the air heater. When it became apparent that the product from the spray dryer was unsuitable for shell production, we tried freeze drying frit precursor droplets as an alternative to drying them in heated air. Freeze drying was successful and appears to be a suitable method for production of monosized frit, however, we have yet to drop the material through the glass shell tower, confirming it will make useable shells.

The glass tower came with an eight-year-old programmable controller-based temperature control system. Without extensive upgrades, the system could only be programmed by the factory. When its main power supply failed, we replaced the programmable controller with a number of stand-alone temperature controllers.

Operation with the new controllers was partially successful. The furnaces could be easily controlled, however, using spray dried frit we could only make small thick walled shells in the temperature range of $700^{\circ}$ to $1375^{\circ} \mathrm{C}$. The furnace temperature was limited by the quartz process tube. We replaced the quartz process tube with a set of alumina process tubes to permit operation up to $1550^{\circ} \mathrm{C}$, the limit imposed by the silicon carbide heating elements.

Glass Shell Deliveries. All glass shell deliveries in FY93 were made from stock. The first delivery of 40 shells was made in October 1992 and the second delivery of 60 shells was made in March 1993. Prospective batches were first located in the inventory records and then examined under an optical microscope to verify that the batch had the potential to provide shells with the requisite properties. In general, the shells were heavily weathered and only a fraction of the potential batches were worth processing further. Selected batches were sieved and washed to remove weathering features and to provide clean shells with the required outside diameter. Individual shells were selected to meet the delivery requirements using optical and interference microscopy to measure shell diameter, sphericity, wall thickness, and wall uniformity.

\subsubsection{LLO4 COMPOSITE POLYMER CAPSULES}

The first part of this task was to make production-ready key capsule fabrication and characterization capabilities. The second part was to mesh the individual capabilities into a state-of-the-art production process and deliver high-quality composite polymer capsules. 
Polystyrene Shell Process Improvement. We were production-ready at the beginning of FY93. We examined a piezoelectric droplet-on-demand generator in hopes of blowing shells with smaller standard deviations for diameter and wall thickness. For the initial testing, we examined the mass distribution of the droplets the generator produced. We used tower conditions that would make beads rather than shells, since mass is easier to determine for beads. We found that the bead diameter distribution was multi-modal, generally with three distinct peaks. With low-viscosity fluids such as water, the mass distribution was very tight. With high viscosity fluids such as polymer capsule solutions, the mass distribution was broad due to the droplet generator's unstable operation and need for continuous tweaking. Although this study indicated we should continue using the traditional droplet generator for shell production, with a more stable design, the piezoelectric droplet-ondemand generator may yet prove to be viable.

GDP Coater. We found optimal conditions for depositing high-quality polymer (CH) coatings on polyvinyl alcohol (PVA) coated shells. Typically, about two thirds of the shells put into the GDP coater had excellent surface finish and the desired coating thickness at the end of the run.

PVA Drop Tower. We developed our PVA drop tower to produce high-quality PVAcoated shells. Our yield of good quality coated shells was consistently less than $1 \%$. The major defects in the PVA coatings included: strings of shells stuck together, small debris, and bubbles. We have continually examined and modified our equipment and process control with the result that our yield was improving by the end of FY93.

Dlagnostic Gas Filling of Plastic Shells. We completed the gas fill system in February. The system has four small fill chambers each of which can be isolated from the others. Two of the chambers can each hold two shell-filled capillaries and the other two chambers can hold one capillary each, for a total of six capillaries. We use a $21 \%$ argon in deuterium mixture for fills. The amount of argon entrapped in the PVA-coated shells according to pressure measurements made with the X-Ray Fluorescence (XRF) system at LLNL was nearly always less than our calculations predicted. We are working with LLNL staff to resolve this discrepancy.

Production. We delivered 20 capsules of one type and 31 capsules of another in response to a request for 20 capsules from each of the two types. The capsules were 
characterized by LLNL and found to meet all their requirements. Although the argon content measured was less than expected, the capsules were still useful to LLNL.

\subsubsection{LLO5 CHARACTERIZATION DEVELOPMENT}

LL05 was a multi-part task grouped under the general heading of Characterization Development. As part of this task we developed a calorimetric technique to determine the amount of hydrogen contained in ICF capsules. We investigated the applicability of a spherical interferometry technique to the measurement of surface roughness on ICF capsules. We constructed a cryogenic insert to do liquid layering experiments. We started work on thermal and fluid mechanical modeling of liquid layers. That work will continue into FY94. We also constructed an automatic capsule sorter to help select drop tower polystyrene shells for PVA overcoating.

We had good results using the calorimetric fuel measurement technique and the calorimeter we designed and built for it. We measured the dew point of an $800 \mu \mathrm{m}$ shell having a nominal fill pressure of 50 atmospheres of $D_{2}$. Our measurement gave a fill pressure of 47.4 atmospheres. The standard optical dew point method gave a comparable measurement of 49.3 atmospheres. In FY94, we expect to improve the calorimetric model to better represent the data and to investigate how well this technique works near the critical point. Our investigations indicated that calorimetry becomes more difficult in the critical region due to increased thermal time constants of the hydrogen fluid. It will be useful to know how close to the critical point we can reasonably expect to make a measurement.

Our investigation of the application of spherical interferometry for ICF capsule surface measurement indicated that the technique has a resolution comparable to that of Atomic Force Microscopy (AFM). Our results were not optimal because the LANL WYKO interferometer used for this investigation was not designed to work with millimeter-size, transparent, hollow spheres. The surface maps we generated for transparent capsules contained spurious features due to reflections from inner capsule surfaces. Therefore, after our initial experiment, all further experiments were performed using opaque spheres. Further work remains to be done on a software algorithm to convert raw surface data files into a $4 \pi$ map of the surface.

We began an experiment to determine the physical limits of the thermal gradient liquid layering technique. Our primary goal is to measure the thickness of a liquid layer as a 
function of the temperature gradient placed across a shell. We wish to determine the maximum practical layer thickness one can obtain with this method. This experiment differs from previous measurements in the method used to create the temperature gradient. Previous experiments have used devices such as thermally regulated gas jets aimed at the shell or thermal gradients imposed upon a surrounding helium atmosphere. We use a heater and thermometer assembly placed directly on top of the shell and attach a cold finger to the bottom of the shell. This arrangement is not expected to provide ideal symmetry for liquid layering, but it is simple, gives very good thermal contact to the shell, and allows us to directly measure the temperature gradient across the shell.

Preliminary results have been obtained for our thermal and fluid mechanical modeling effort using a commercial differential equation software package. We made a simple model of heat and mass flow in a $D_{2}$ gas bubble maintained in $D_{2}$ liquid with a predefined thermal gradient. We also modeled the conduction of a multi-layered shell of polystyrene, liquid $D_{2}$, and $D_{2}$ vapor in a helium gas atmosphere. This was also done with a predefined thermal gradient.

We completed the construction of an automatic shell sorter. The shell sorter can sort shells by size, roundness, and wall thickness. Its shortcoming is the speed at which these tasks are accomplished. As currently configured, it can work unattended overnight to recover a few hundred shells from an unsorted batch of several thousand shells. A large percentage of the time needed to do a sort is consumed by computer processing of the digital shell images. We expect to acquire a faster computer by FY95 that can increase the data processing rate by a factor of two to four.

\subsubsection{LLO6 MICROMACHINED TARGET COMPONENT FACILITY}

In the Micromachined Target Component Facility task we procured, installed, and set up a Micromachining Facility at General Atomics. The primary component of this facility is a Precitech Ultra-Precision Diamond Turning Lathe which we received in July, 1993. We then trained personnel in the operation of the micromachining equipment, and once the personnel were trained, we produced a set of precision machined test mandrels to demonstrate the capability of the facility. These mandrels were delivered to LLNL for capability validation at the end of FY93. 


\subsubsection{LLO7 TARGET COMPONENT ELECTROPLATING FACILITY}

In the target component electroplating facility task, we procured and installed equipment for electroplating of LLNL specified components. In doing so, we copied as closely as possible the electroplating equipment at Rocky Flats. We then trained personnel in the operation of the facility and subsequently electroplated a series of specimens to validate the capability of the facility.

\subsubsection{LAO1 COMPOSITE POLYMER CAPSULES}

In June, we delivered 14 capsules in response to an urgent request for 10 capsules with standard specifications. We delivered the capsules within two weeks of receiving the request.

We delivered 36 capsules called "Exacts" for the "Symimage" experiments. The experimenter requested capsules of nearly identical diameters and wall thicknesses. Four capsules were required, one of each combination of Argon/No Argon and Chlorine/No Chlorine. The four capsules had to be within $5 \mu \mathrm{m}$ of each other for inside diameter, within $0.5 \mu \mathrm{m}$ of each other for the polystyrene plus PVA thickness, and within $1 \mu \mathrm{m}$ of each other for the GDP layer thickness. These are very restrictive specifications, about an order of magnitude tighter than usual.

In July, we prepared and characterized nearly 50 batches of chlorinated and nonchlorinated polystyrene shells before we found enough batches that met the tight wall thickness and inner diameter specifications. In August, we coated the shells with PVA. In September, we GDP coated the shells with $\mathrm{CH}$, fully characterized them, and delivered 36 capsules. Matching the four best capsules was done at LLNL; many matches were found.

\subsubsection{LAO2 ONSITE SUPPORT FOR LANL}

This task included the services of two individuals: a scientist onsite at LANL to support cryogenic target development activities, primarily by examining B-layering; and a technician onsite at LLNL to perform final assembly of LANL NOVA targets. Late in FY93, a few miscellaneous activities were added to the task.

Cryogenic Target Development Actlvities. In FY93, John Simpson performed cryogenics experiments at LANL focused on the examination of deuterium-tritium (DT) solid layers 
inside a copper cylinder having an inner-diameter of $2 \mathrm{~mm}$, and on the characterization of these layers for surface roughness. This work included: design and installation of an electronic system used to synchronize Charge-Coupled Device (CCD) image acquisition to cryostat motion; purchase and installation of a new flash illuminator used to minimize exposure times and thermal perturbations of the DT solid layer; installation of a new instrument bus control board and data acquisition software to allow automated image acquisition, including automated DT cell temperature control; and data reduction and analysis of both experiments using new analysis software written by Evan Mapoles of LLNL.

The first experiment produced a solid DT layer $125 \mu \mathrm{m}$ thick and ran for about 46 hours. The final layer had a surface roughness of about $1.8 \mu \mathrm{m}$ root-mean-square (rms). The DT surface finish continued to improve throughout the experiment, showing the process had not yet come to equilibrium. A new nonlinear fitting routine in the analysis software reduced edge finding scatter by a factor of five, thereby improving the resolution of surface finish details.

The final DT layering experiment in FY93 produced a layer $139 \mu \mathrm{m}$ thick and used new electronics to synchronize image acquisition to cryostat motion as well as a new flash unit for the cell illumination system. Jitters in image position and focus were significantly reduced by synchronization, thereby improving our image resolution. The flash unit, which was synchronized to the camera shutter and replaced the Continuous Wave (CW) incandescent light, gave us the ability to shorten image exposures to $2 \mathrm{msec}$ and dramatically reduced the effect of cryostat induced image blurring during the exposure interval. The short illumination intervals also virtually eliminated thermal perturbation effects of the light source on the DT solid layer. For this experiment, we made surface roughness measurements at three temperatures and achieved a surface finish of $1.7 \mu \mathrm{m} \mathrm{rms}$. Data also indicated that we may have had a deuterium rich fuel mixture, instead of the expected 50-50 mix.

Target Assembly Activities. We hired a technician, Kett Gifford, who started in late October. He was trained at LANL and LLNL. He assembled and characterized various classified and unclassified targets, characterized glass shells, and prepared Formvar films and other target components.

Miscellaneous Activitles. Two activities were added to this task in late FY93. In one, Jon Larsen of Cascade Applied Sciences, developed software for operation of the 
Powellscope at LANL. In the other, Dave Diffenderfer of WJSA, performed scanning electron microscopy (SEM) analysis on a 16-hole $x$-ray aperture plate.

\subsubsection{LAO3 CHARACTERIZATION OF OPAQUE BETA-LAYERED TARGETS}

This task was centered on two projects. One is the development of an instrument for examining targets using Resonant Ultrasound Spectroscopy (RUS). The other is the development of 1-dimensional (1-D) spatially resolved Nuclear Magnetic Resonance (NMR) for examining beta-layered targets and the beta-layering process for isotopic symmetry.

The RUS instrument was enhanced in a number of ways and initial testing begun. We replaced the prior ultrasound transducers with new very compact transducers arranged in a tetrahedral configuration. The cryostat was modified to provide electrical isolation to the transducers and their electronics from the cryostat. To eliminate low frequency noise induced by the cryostat vibration, a noise reduction circuit was designed and tested. The data acquisition and control system hardware and software were upgraded. The RUS testing was begun using a stainless steel ball.

The 1D-NMR instrument development started this year. Neil Alexander built prototypes and collected data on hydrogen to gain experience in preparation for examining DT systems. A prototype double resonance (D and T frequencies) NMR probe was successfully built. A proton NMR probe was outfitted with several designs of magnetic field gradient coils that produce the one dimensional spatial resolution. This probe was used to obtain 1-D proton projection images of several samples. These included liquid acetone, water, and gaseous molecular hydrogen together with solid molecular hydrogen. A number of components for the DT probe were purchased, including a continuous flow liquid helium cryostat, temperature controller, sapphire sample cell, and plated Radio frequency (RF) transmission line tubing.

\subsubsection{LAO4 MICROENCAPSULATED SHELLS}

The objective of the Microencapsulated Shells task was to continue the development of techniques for efficient production of multi-millimeter, high quality, polymer shells. Both theoretical and experimental techniques were applied toward this end. We formulated a mass transfer model of the solidification process to better understand the transport phenomena. In addition, we measured phase equilibrium of the mixture of polystyrene, 
water, and methylethyl ketone using thermal optical analysis. We optimized the organic solution process by statistical experimental techniques and improved controlled-mass microencapsulation shell handling methods and system capacity.

The first step toward a better fundamental understanding of microencapsulation was the formulation and implementation of a mass transfer model of the solidification process. We developed the governing equations from first principles and selected appropriate constitutive equations to obtain meaningful results. After formulating the problem, we solved the equations using the Galerkin Finite Element method and completed a model for a single-solvent polystyrene solution.

To optimize the organic solution composition, we applied statistical experimental methods to the complex emulsion method of microencapsulation. Solvents for the studies were methylethyl ketone, 1,2-dichloroethane, benzene, and styrene. The responses we measured were: shell diameter, wall thickness, vacuoles, sphericity, and wall uniformity. After examining the results, it appeared that the optimum composition for any given variable was outside the range of the experiments. Therefore, we recast the results in terms of solubility parameters. Applying solubility parameters to the results allows us to expand our predictive capability to solvents beyond the limited number tested.

We made many improvements to the controlled-mass microencapsulation system. The most important advance was in the actual design of the triple-orifice droplet generator. Previously, assembly was tedious and difficult. Now, with our new design, the whole assembly simply screws together and the precisely machined parts act to align the orifices concentrically. The next improvement was the column configuration. We changed the column shape from a cylinder to a cone. This improved the suspension of shells in the column and increased the yield of any given run. We also increased the capacity of the equipment by assembling a parallel fluid handling system. After completing these improvements, we confirmed the controlled-mass nature of the triple orifice system from product shells. We were able to consistently produce shells of $1 \mathrm{~mm}$ size, $5 \mu \mathrm{m}$ wall thickness, with narrow distribution ( $\pm 0.15 \mathrm{~mm}$ diameter and $\pm 2 \mu \mathrm{m}$ wall). The next step is to improve sphericity and concentricity to target quality. 


\subsubsection{LAO6 SMALL GLASS SHELL DELIVERIES}

The goal of this task was to manufacture and deliver approximately 50 high quality glass shells to meet LANL's target requirements. Before shell manufacturing could begin, we needed to complete the shakedown of the glass shell tower and develop glass frit production capability. In the interim, while awaiting completion of the production facilities, we were to sort through available stock to find suitable shells for delivery.

All glass shell deliveries were made from stock. The first delivery of 21 fully characterized shells and 30 witness shells was made in January 1993, and the second delivery of 20 fully characterized shells and 30 witness shells was made in July 1993. Prospective batches were first located in the inventory records and then examined under an optical microscope to verify that the batch had the potential to provide shells with the requisite properties. In general, the shells were heavily weathered and only a fraction of the potential batches were worth processing further. Selected batches were sieved and washed to remove weathering features and provide a clean cut of shells with the required outside diameter. Individual shells were selected to meet the delivery requirement using an optical and interference microscopy to measure shell diameter, sphericity, wall thickness, and wall uniformity.

The witness shells were selected, sieved and washed in the same manner as the deliverable shells, but were not characterized further. All shells were delivered in egg crates, ready for tritium filling.

\subsubsection{NRO1 NAVAL RESEARCH LABORATORY (NRL) TARGET DEVELOPMENT}

At the beginning of FY93, we selected three methods of producing thin, uniform films of $\mathrm{CH}$ polymer for study and evaluation. These were: parylene coating of flat glass substrates, coating of a glass substrate pulled from a solution of a polymer at a uniform rate, and polystyrene film casting from a solution on a still water surface.

We demonstrated the capability to produce high quality $\mathrm{CH}$ films. The film quality produced in the parylene system was better than the $10 \mathrm{~nm}$ roughness required for the NRL CH-film targets. The parylene system also demonstrated that films of $\mathrm{CH}$ with a doping of chlorine could be produced. Further work on the parylene system is required to demonstrate 
the capability of producing high quality parylene films in the 45 to $100 \mu \mathrm{m}$ thicknesses required for the NRL NIKE targets.

A system was assembled by which clean, glass microscope slides can be pulled at a uniform rate from a solution of polystyrene in suitable solvents to produce films of polystyrene. However, because of the success of the cast film deposition method, the pulled film technique was not pursued beyond the demonstration phase.

We used two techniques in our attempts to attain the demanding NRL surface finish specifications. We began with the historical technique of heating the polymer between two optical flats. However, as we improved the surface finish, the polystyrene film became more difficult to remove from the flats without damaging the film. The obvious answer was to cast the film on a liquid substrate. High purity water proved very successful. We overcame film curvature caused by water evaporation and meniscus effects by casting large ( 6 inch) diameter films and by using high humidity environments. We experimented with various molecular weights of polystyrene and various solvent solutions to enhance production, handling, and target assembly processes. With the use of a clean room, we have been able to cast sets of films meeting the stringent surface and volume specifications for the NIKE CHfilm targets.

In a strongly interactive design effort with NRL staff members, we designed a $\mathrm{CH}$-film target mount which will satisfy the many requirements associated with the NIKE laser system. The mounts were made from a polycarbonate material by using inexpensive, injection molding techniques. Dimensional tolerances of $\pm 25 \mu \mathrm{m}$ were maintained in the injection molding process.

We used optical microscopy, interferometry, and photon tunneling microscopy as the primary techniques for characterization of the polymer films for NIKE targets. Prototype CH-film targets were assembled and delivered to NRL for evaluation by the NIKE program staff. We assembled $\mathrm{CH}$-film targets on a routine basis and are storing them to be used as needed by the NIKE program.

We designed and fabricated much of the apparatus necessary to begin studies on the production of planar layer cryogenic targets for the NIKE laser experiments. Temperature measurement and control systems and a high vacuum pumping station have been purchased and a liquid helium dewar has been ordered. 


\subsubsection{SLO1 FABRICATION OF FOILS AND FILTERS}

Work on this task continued uninterrupted from last year with production of various types of flat films and filters for Sandia National Laboratory (SNL). Altogether more than 1100 films and filters of 11 different general types were fabricated, characterized and delivered under this task in FY93. In addition, an engineer assigned full-time at SNL fabricated another 640 films and filters using equipment at SNL.

\subsubsection{URO1 OMEGA UPGRADE CRYOGENIC TARGET SYSTEM}

During FY93, the GA/WJSA team worked on layering and characterization of cryogenic ICF targets and the system engineering for the OMEGA Upgrade cryogenic target delivery system. We evaluated thermal gradient layering of liquid hydrogenic targets for its suitability for production of OMEGA Upgrade cryogenic targets. This involved support of the experimental layering activities at LLNL and phenomenological modeling of the thermal gradient layering of $D_{2}$.

Two Mach Zehnder interferometry systems were borrowed from the University of Rochester, modified and evaluate for their ability to characterize millimeter-size transparent shells with hydrogenic fur: layers up to $100 \mu \mathrm{m}$ thick.

We developed a Research and Development (R\&D) program plan for determining acceptable cryogenic target layering techniques. The plan addressed issues that need to be resolved for both solid and liquid layering as well as the development of characterization techniques.

System engineering activities included: a design and proof-of-principle testing of the high pressure (1500 atmosphere) cryogenic booster system for the University of Rochester DT fill station, a design for the method for handling (removal and transport from the DT fill station) of cryogenically cooled DT-filled targets, and a design for cryogenic storage of DT and $D_{2}$ filled targets. We achieved pressures of 1600 atmospheres in a custom designed gas intensifier system. We constructed an apparatus that allows us to test vacuum seals of various designs for their ability to be made and broken at cryogenic temperatures. Several prototype target manipulators were built and operated in a test stand.

We assisted the University of Rochester's target support efforts, proposing a tripod target mounting technique and providing analysis of potential vibrational modes. 
We also developed a program plan for designing and constructing the OMEGA Upgrade Cryogenic Target Handling System. This included a Work Breakdown Structure (WBS), a project schedule, and a project cost estimate based on the WBS.

\subsubsection{URO2 LLE MATERIALS DEVELOPMENT}

The LLE Materials Development task had two components: synthesis of poly[perdeutero(trimethylsilyl)styrene], and synthesis of a polymer which is opaque at $350 \mathrm{~nm}$ and transparent at visible wavelengths.

Anthracene was found to be a candidate chromophore for the ultraviolet/visible optical characteristics desired. We sought to replace the phenyl ring of styrene with anthracene, to make vinylanthracene, and to replace the phenyl rings of parylene with anthracene to make $(2,2)(9,10)$ anthracenophane. The UV-visible spectrum of poly(vinyl anthracene) was measured in solution, and found to have the optical characteristics desired. At $350 \mathrm{~nm}$, a $10 \mu \mathrm{m}$ film would have a transmission of $10^{-8.56}$, only $30 \mathrm{ppb}$ of light will be transmitted. Vinyl anthracene was difficult to polymerize to high molecular weight, and shells made from the polymer were mechanically weak. Anthracenophane was synthesized by literature methods. It could be vaporized at $150^{\circ}-200^{\circ} \mathrm{C}$, and cracked to the reactive monomer at $300^{\circ} \mathrm{C}$, to make a deposited poiymer.

The synthesis of perdeuterated trimethylsilylstyrene was attempted from deuterated styrene. The synthesis route sought to make perdeuterated bromostyrene in four to five steps. The yield of product in several steps was lower than expected, and at one step, no product was obtained. However, we identified an impurity and used it to continue toward the product. Some 10-20 grams of perdeuterated trimethylsily styrene was ultimately produced. 
SECTION 2

CAPABILITIES DEVELOPMENT 


\section{CAPABILITIES DEVELOPMENT}

A significant portion of our activities was devoted to developing fabrication and characterization techniques for the production of ICF target components.

\subsection{SHELL FABRICATION}

Thin walled glass and polymer shells are routinely used as fuel containers in ICF implosion targets. Polymer shells, with their lower atomic mass and thus lower susceptibility to Rayleigh-Taylor instabilities, are of primary interest as modern ICF targets. Glass shells formed the basis of early ICF experiments and are still required for laser diagnostics. Both glass and polymer shells are made in drop towers. Whereas glass easily retains deuterium and tritium fuel gases, polystyrene (PS) shells require an additional coating of polyvinyl alcohol to form useful targets.

\subsubsection{POLYSTYRENE SHELL TOWER}

The Capability Activation task was completed in the key areas of coatings and characterization so that production and delivery of ICF capsules and components to the national ICF Laboratories could commence.

The polystyrene shell tower is a 20 foot tall piece of six inch pipe, heated over the top twelve feet. Droplets of polystyrene dissolved in methylene chloride are dropped into the top of the tower. As the droplets fall, the solvent evaporates and blows the polystyrene into hollow shells. The standard means of droplet formation is via Rayleigh breakup of a laminar jet whereby a mechanical transducer induces the desired disturbance frequency on a jet whose flow rate is determined by a syringe pump. In FY93, we developed an alternative droplet generator that uses piezoelectric material.

Under a negative electric field, a piezoelectric material increases in thickness in the direction of the field while decreasing in the dimensions normal to the field so as to maintain constant volume. The piezoelectric droplet generator shown in Fig. 2-1, has electrodes on the 
inside and outside surfaces of the piezoelectric cylinder. If the outside electrode is maintained at ground potential and a negative voltage is applied to the inside electrode, the cylinder wall thickens, its mean diameter remains constant (decreasing the inside diameter), and the cylinder height decreases. The result is a decrease in the internal volume of the cylinder. The change in volume can be calculated from the properties of the piezoelectric material and the geometry of the cylinder.

We characterized our piezoelectric droplet generator to determine its operational characteristics. Figure 2-2 shows the calculated and measured volume displacement of the generator as a function of the imposed voltage. The volume displacement was found by measuring the linear displacement of a liquid meniscus in a capillary tube connected to the generator. The measured volume displacement for a constant voltage was close to the predicted value, however, when we used a $30 \mathrm{~Hz}$ square wave, the displacement was only a quarter of its steady-state value.

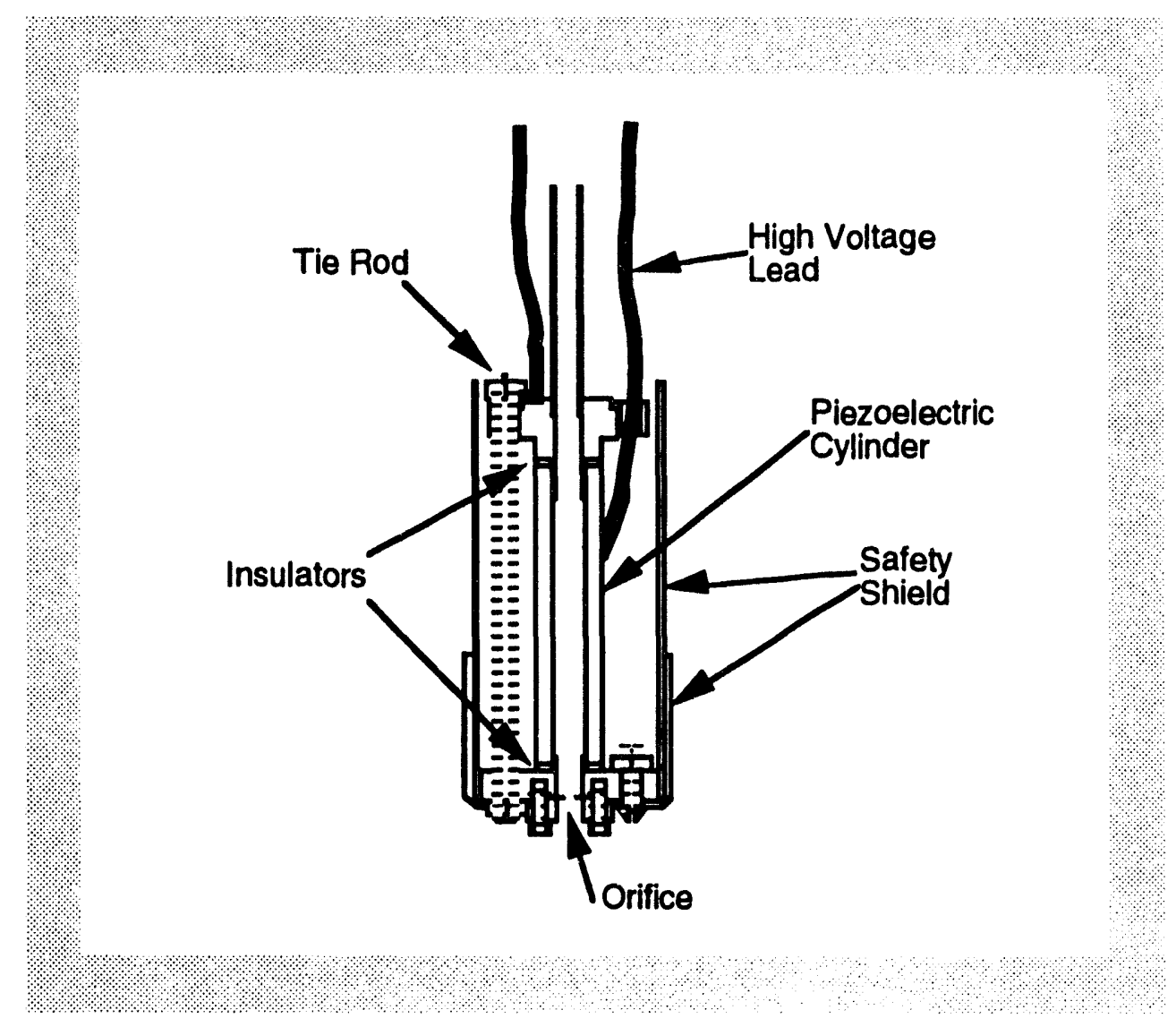

Fig. 2-1. The plezoelectric droplet generator. 


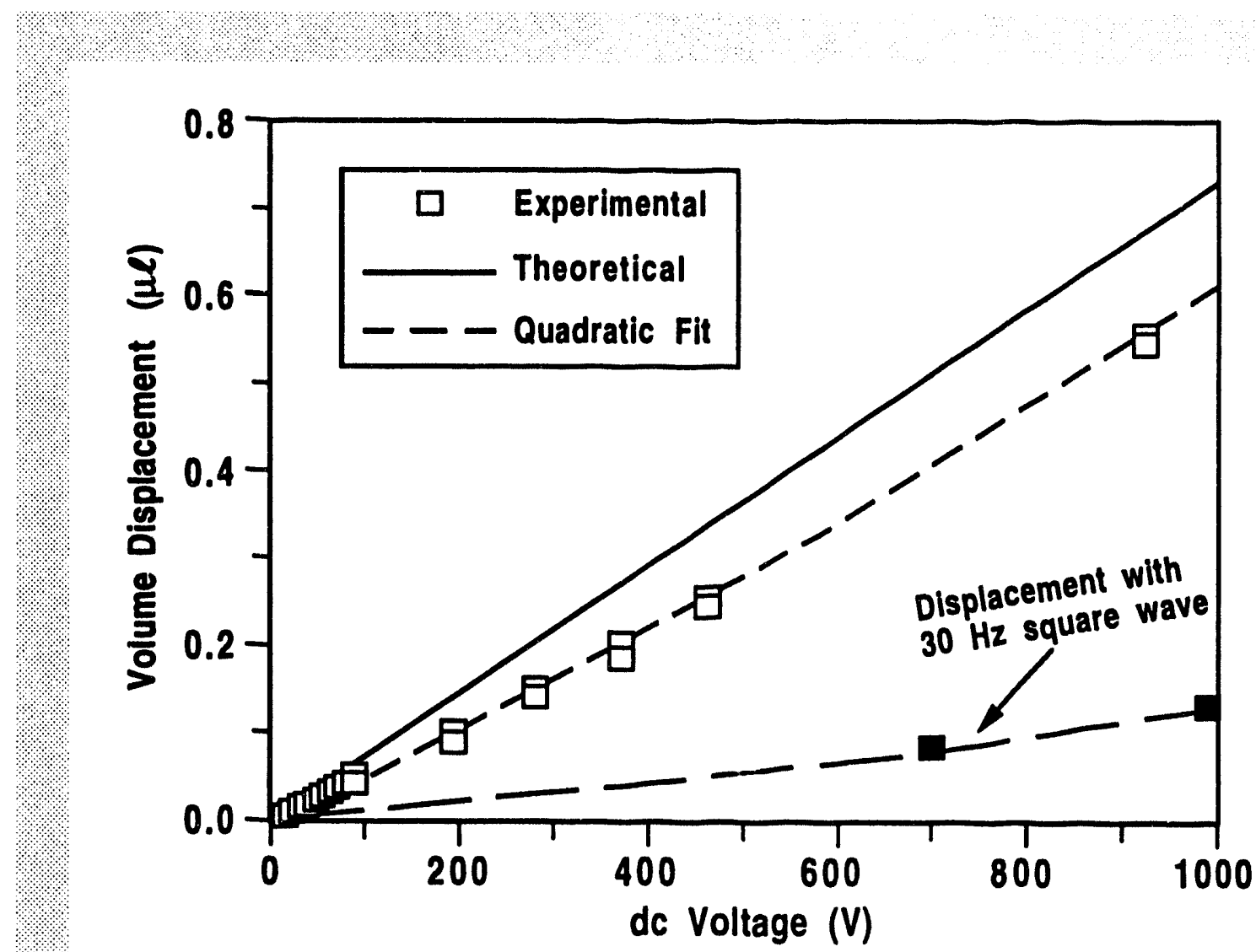

Fig. 2-2. Volume displacement of the piezoelectric droplet generator.

We originally expected that the droplet generator would be capable of operating in a deterministic mode wherein the volume of the droplet would be uniquely fixed by the voltage imposed. The lower than expected dynamic displacement measurements indicated that deterministic operation would be difficult to achieve. The most probable cause of the low dynamic displacement was the presence of a compressible material within the cylinder volume, the most obvious being small gas bubbles. Although every effort was made to remove air bubbles, it was possible that some small air bubbles were still present. A second source of compressible material is the rubber gasket material used to seal the piezoelectric cylinder to the end flanges. A third source is the liquid in the cylinder. Although we normally consider liquids to be incompressible, the compressibility of a liquid is significant when compared to the volume of a droplet. The droplet volume required for shell production is in the range of 0.1 to $0.3 \mu l$, but, a one atmosphere 
pressure change in the droplet generator cylinder results in a volume change of $4.0 \mu \ell$. When the droplet generator was operated at voltages corresponding to those predicted for the deterministic mode, no droplet formation was observed. In order to form droplets, it was necessary to use voltages at least an order of magnitude greater.

Although the piezoelectric droplet generator does not appear to be operable in the deterministic mode, it can operate in a dynamic mode. In this mode, the droplet volume is determined by both the imposed voltage and the pulse width. When operated in this mode, the generator gave erratic results and the voltage required to obtained droplet formation varied widely from run to run. At times the droplet size distribution was unimodal, but more often it was not. The presence of air bubbles was the probable reason for the lack of reproducibility.

For further information, please contact Dr. L. Brown (GA) or Dr. C. Hendricks (WJSA).

\subsubsection{PVA COATINGS}

Polystyrene shells are made impenetrable by gases by coating them with PVA. The polystyrene shells are coated with an aqueous solution of PVA and dropped through a heated tower where the PVA solution dries to form the permeation barrier coating. Any desired diagnostic gas must be inside the shells before the PVA coating is applied.

The PVA coating tower, a 20 foot tall by 18 inch diameter heated tube, was completed and put into service this year. The heating system consists of ten convection ovens, each two feet tall. The ovens are physically, but not thermally, isolated from each other by a sheet metal partition. A fan within each oven section mixes the air and directs it past a heating element to produce a constant air temperature within the section. The air temperature within each zone is monitored by a temperature indicating controller.

One section of the tower was instrumented with thermocouples to permit measurement of the temperature around the circumference of the process tube. Figure 2-3 indicates the temperature of the process tube without the fan running, with the fan operating as originally designed, and after airflow modifications were made to improve temperature uniformity in the oven.

We obtained best temperature uniformity in the oven by adding a second fan to the blower and connecting a duct from the coolest corner of the oven to the blower. A small 
Fig. 2-3. Tomporature uniformity of PVA tower as a function of fan configuration.

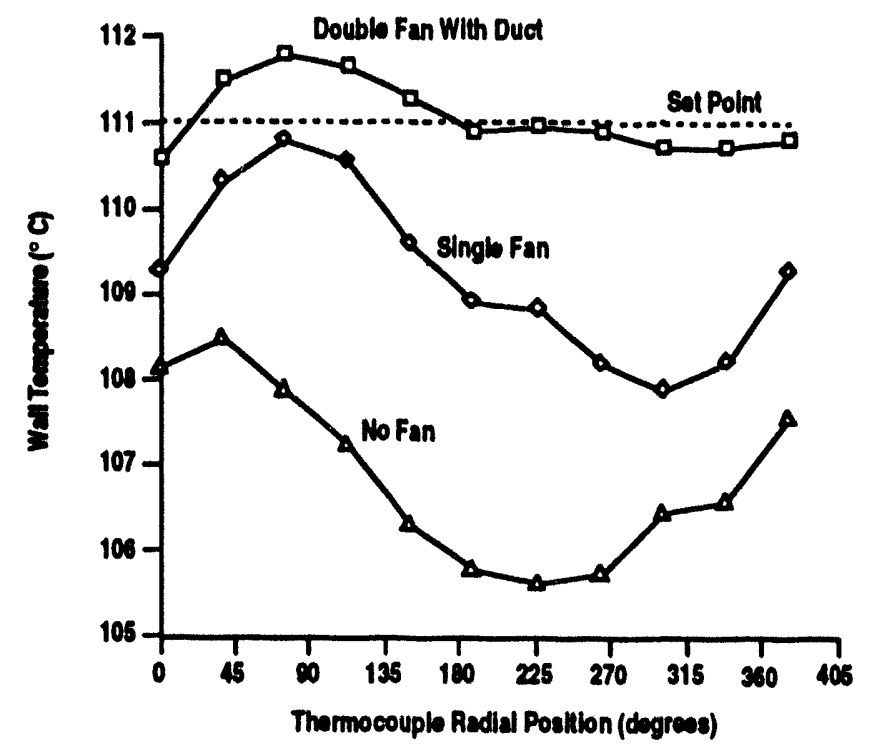

secondary opening in the duct was located in the back corner of the oven. Figure $2-4$ shows the location of the duct within the oven. With the modifications, the circumferential temperature is uniform to better than $\pm 1^{\circ} \mathrm{C}$.

For further information, please contact D. Husband or W. Baugh (GA).

\subsubsection{FRTT PRECURSOR}

We refurbished a spray dryer obtained from LLNL to produce monosize frit particles for the manufacture of glass shells. Several batches of frit were produced using the spray dryer, however, its heating system contaminated the frit with metallic oxide particles. The heating elements of the air heating system sloughed off rust particles which were entrained in the hot air used in the spray dryer. In addition, the frit particles were friable and much of the product was not monosized due to particle breakage.

Freeze drying of droplets of frit precursor solution (produced by a droplet generator) gave better results than spray drying. When we freeze dried droplets in liquid nitrogen, they were transformed into clean, uncontaminated, monosize frit particles. The particles are hygroscopic and must be stored at low humidity. 


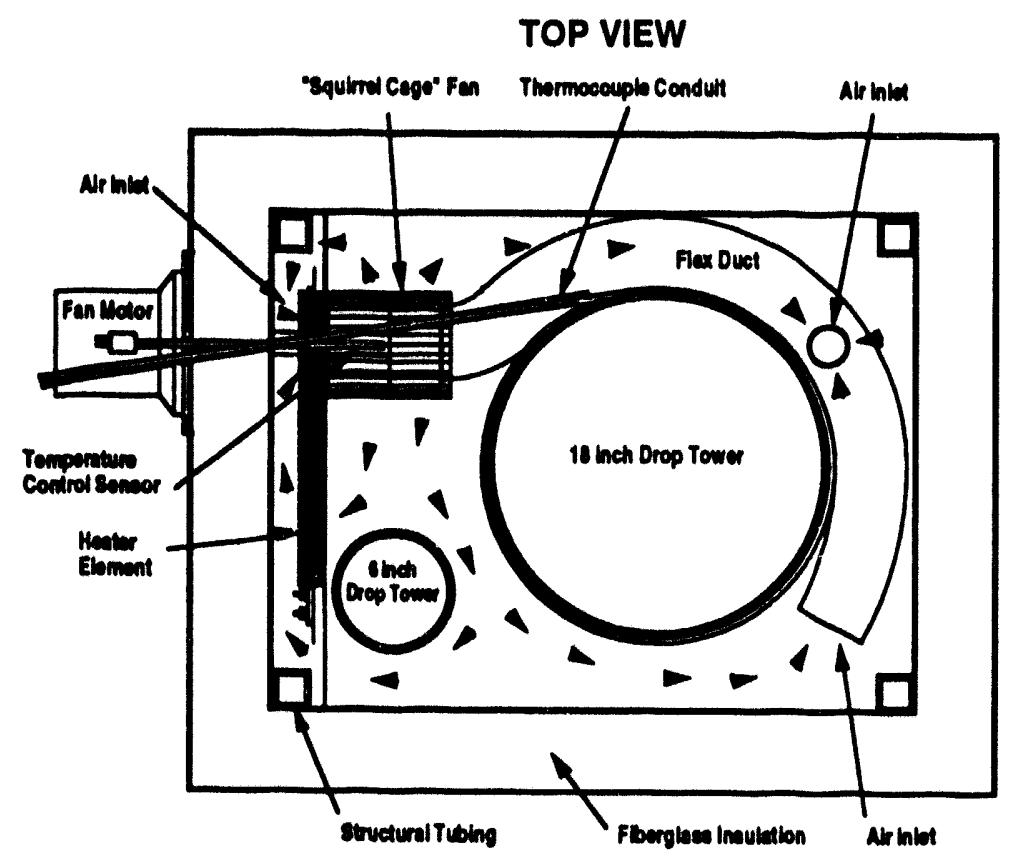

Fig. 2-4. Top viow of modified PVA tower oven.

For further information, please contact Dr. C. Hendricks (WJSA).

\subsubsection{GLASS SHELL TOWER}

The glass shell tower was operated using frit produced by the spray dryer. The quartz process tube limited the operating temperature to $1375^{\circ} \mathrm{C}$. At this temperature, only a few small thick-walled shells were produced.

The quartz process tube was attacked by alkali vaporized from the frit even though the process tube was protected with a sacrificial lining of one foot sections of quartz tubing. The alkali vapors attacked the process tube at each joint in the sacrificial lining and even welded the lining to the process tube in places. While trying to remove the sacrificial lining, we broke the quartz tube. Although only a small fraction of the wall of the quartz tube was damaged, we did not to attempt to repair it because preferred, high-temperature, alumina process tubes had arrived. 
The alumina tubes were not available in the length required for the glass tower. We therefore installed a pair of tubes such that the smaller upper tube slipped into the lower tuhe with an overlap of about one foot. The top tube is suspended from an upper ball joint and the lower tube is supported from a ball joint clamped to the tube near its bottom. The ball joints are stainless steel and rest in conical holes in support plates that are positioned with jack screws to align the process tubes. At the end of FY93, we had configured the glass shell tower with the alumina tubes but had not yet tested it at temperature.

For further information, please contact Dr. L. Brown (GA).

\subsection{COATINGS FABRICATION}

A polymer coating must be applied to the shells to serve as an ablator.

\subsubsection{GDP COATINGS}

Having completed capabilities validation in FY92 using the first GDP coater, assembled at GA with an LLNL helical resonator, we were ready to produce capsules according to customer specifications. We coated capsules for three customer orders in FY93. Each production coating run successfully produced the desired coating within customer specifications for thickness and surface finish. Table 2-1 shows the number of capsules ordered, the required tolerances, the time elapsed between the receipt of the substrates and the

\section{TABLE 2-1}

GDP Phoduction Coating Run Statistics

\begin{tabular}{|c|c|c|c|c|c|c|}
\hline $\begin{array}{c}\text { OrOER. } \\
\text { No. }\end{array}$ & $\begin{array}{l}\text { CAPSULES } \\
\text { ORDERED }\end{array}$ & 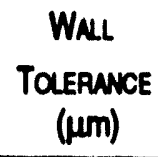 & $\begin{array}{c}W_{\max }-W_{\min } \\
\text { TOLERWNCE } \\
(\mu \mathrm{m})\end{array}$ & $\begin{array}{l}\text { GDP RUN } \\
\text { DuPATION } \\
\text { (DAYS) }\end{array}$ & $\begin{array}{l}\text { CAPSULE } \\
\text { YIELD } \\
(\%)\end{array}$ & $\begin{array}{l}\text { CAPSULES } \\
\text { Delinered }\end{array}$ \\
\hline $1 A$ & 20 & \pm 5 & \pm 5 & 7 & 75 & 20 \\
\hline $1 B$ & 20 & \pm 5 & \pm 5 & 8 & 75 & 31 \\
\hline 2 & 10 & \pm 5 & \pm 5 & 5 & 75 & 14 \\
\hline 3 & 4 & \pm 2 & \pm 0.5 & 9 & 66 & 36 \\
\hline
\end{tabular}


completion of the run, the yield of target quality capsules, and the number of capsules delivered for each GDP production coating run.

\subsubsection{Second GDP Coater}

The objective of this portion of the task was to produce target quality coatings using the second GDP coater assembled at GA. (This coater employs a helical resonator also assembled at GA.) In early April, the second coater produced a good quality $43.5 \mu \mathrm{m}$ thick $\mathrm{CH}$ coating on PVA-coated polystyrene capsules, as shown in Fig. 2-5. However, subsequent runs occasionally produced coatings with an unacceptable number of domes. During the second half of FY93, urgent customer requests for deliverables hampered our ability to conduct validation runs on the second coater. Due to these requests, we had to designate almost all target quality PVA coated shells to production runs at the expense of validation runs on the new coater. PVA coated capsules are needed for validation runs because they behave differently in the coater than uncoated polystyrene capsules [Ref. 2-1]. At the close of FY93, we initiated a series of coating runs which lead to capability validation of the second coater in early FY94.

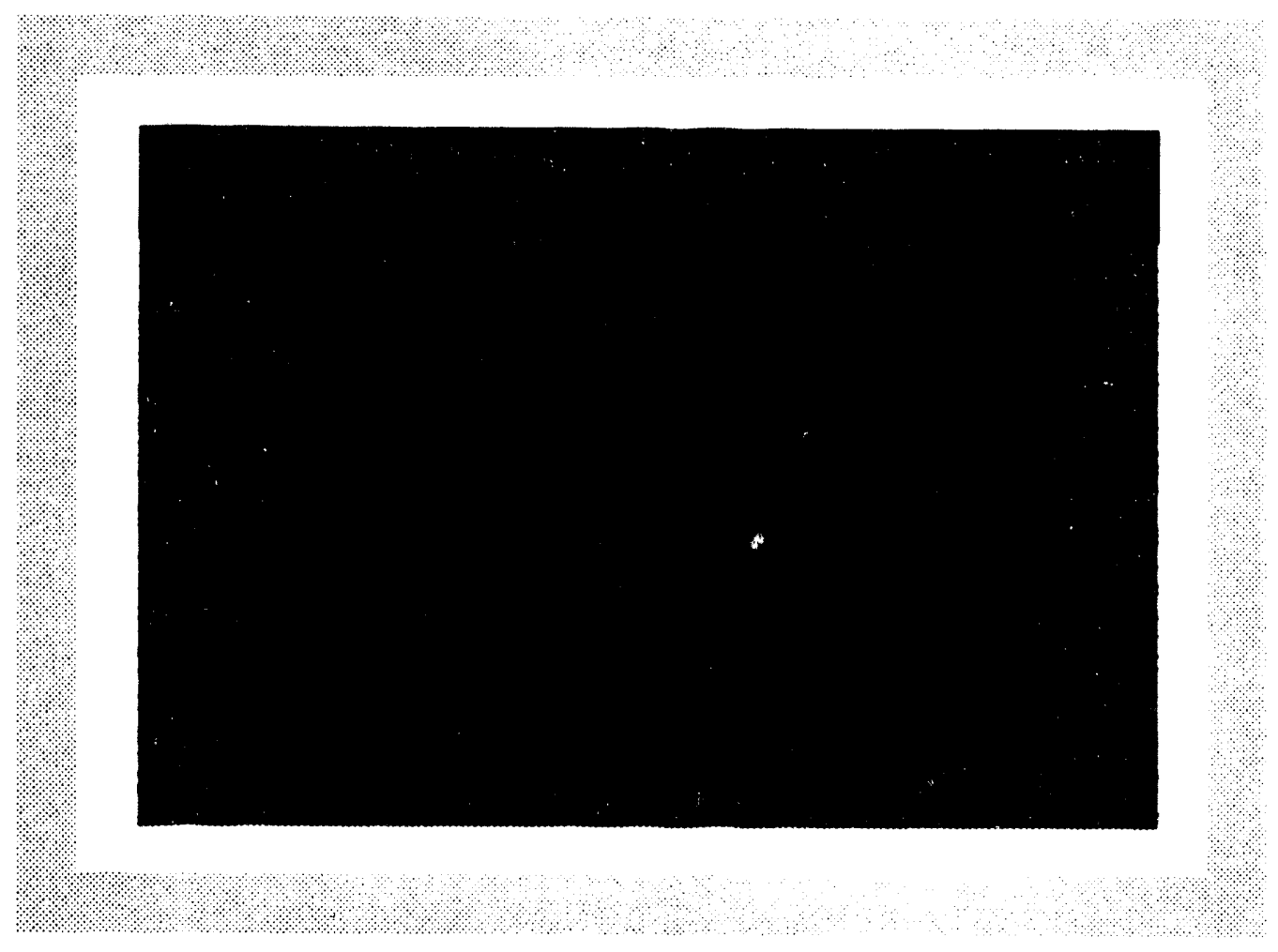

Fig. 2-5. SEM micrograph of the surface of a capsule coated using the second GDP coater 


\subsubsection{GDP Diagnostics}

We developed two diagnostics for our GDP coaters. The fist diagnostic continuously monitors the plasma $\mathrm{H}_{\alpha}$ line emission from the coater. As typical capsule coating runs are several days in duration, a diagnostic that monitors coater operation when no one is present can prove useful. The second diagnostic is an in-situ diameter measuring system that may allow us to determine GDP coating thickness without having to remove shells from the coater and risk contamination.

$H_{\alpha}$ Line Emission. Figure 2-6 shows a trace of an apparent variation in $\mathrm{H}_{\alpha}$ line emission. The monitor detects and records the intensity of light emitted from the plasma tube as a function of time. The high light output recorded at the end of the trace corresponded to a situation where the plasma extended high into the plasma tube, nearer to the detector port. In a run where this condition was persistent, the capsule coating rate was abnormally low and the tube received a thickened coating. This tracing also shows a period of rapid fluctuation in light output preceding the movement of the brightest glowing region into the upper part of the tube. Investigation of this phenomenon may help us understand the operation of the helical resonators.

The operation of a helical resonator is complex. Within the helical resonator coil, the distribution of the magnetic field intensities is a function of frequency. Furthermore, the helical resonators are run near the series resonant frequency. Brusasco and Fergusson have

\section{Relative \\ Intensity}

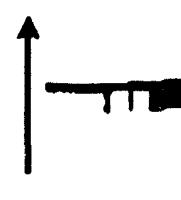

Time (30 min/cm)

Fig. 2-6. $H_{\alpha}$ llne emission of GDP coater over 11.5 hours. 
found that the magnetic field distribution changes rapidly with changes in frequency near this point [Ref. 2-2]. To complicate things even more, the presence of plasma changes the series resonant frequency, as does the position of the RF shield, so that it is difficult to know where one is operating with respect to resonant frequency. At some frequencies near series resonance, the currents in different regions of the resonator coil are nearly equal [Ref. 2-3]. This could give rise to an unstable situation, with the plasma distribution changing in response to small changes in the impedance of circuit elements due to minor changes in the coater environment.

In-Situ Capsule Dlameter Measurement. We have been developing a diagnostic which will measure capsule diameters while the capsules are still in the GDP coater. With this ability, we will be able to stop coating capsules when they have increased in size by the desired amount. This diagnostic consists of a Pulnix camera (Model TM745E) attached to an Optem 6:1 zoom lens. The images this system provides are measured from a monitor using a Techni-Quip digital video caliper (Model T-Q/DMZR-YC). Our goal is to measure coating thickness to within $\pm 5 \mu \mathrm{m}$ using this system.

We determined the accuracy of this system by measuring the diameters of capsules of known size. After incorporating the digital video caliper and using National Institute for Standards and Technology (NIST) traceable spherical calibration standards, we found its accuracy to be better than $1 \%$. Based on this result, we felt that thickness determination by measuring diameter change was feasible and proceeded to determine the accuracy we could achieve when measuring the average coating thickness of a batch of shells.

To make this determination, we measured the diameter of capsules in-situ (in the coater) before and after coating. Since we had no way of labeling capsules within the coater and because the field of view was not large enough to measure all the shells, determining the average coating thickness of the batch posed a problem. Our solution was to use batch sampling to determine the average capsule diameter. The accuracy of a batch average obtained from a sample is dependent on the variability of the sampled population. For this reason, the accuracy of the average batch diameter measurements varied, with the error approaching $5 \%$ in one case. Thus, we decided to use batches sieved to narrow diameter ranges (10 to $20 \mu \mathrm{m}$ sieve cuts). Using this methodology, we were able to measure the batch average diameter to an accuracy of better than $1 \%$. 
We made one batch coating thickness determination this year to test the diameter measurement diagnostic. There was a $9 \%$ difference between the value for the coating thickness we obtained using this diagnostic and the value obtained using the standard tool, interferometry. This was quite reasonable in light of the $1 \%$ error in the before and after diameter measurements from which the coating thickness is calculated.

\subsubsection{Doped Coatings}

We deposited a germanium-doped $\mathrm{CH}$ coating on a silicon wafer and glass slide by adding tetramethyl germanium (the dopant suggested by Steve Letts of LLNL) at a rate of $0.03 \mathrm{sccm}$ to a feedstock mixture of $0.14 \mathrm{sccm}$ trans-2-butene and $10 \mathrm{sccm}$ hydrogen. We used a coating chamber pressure of $75 \mathrm{mTorr}$ and an RF power setting of $10 \mathrm{~W}$. These conditions produced an $18.5 \mu \mathrm{m}$ thick coating in 24 hours.

Although some $2 \mu \mathrm{m}$ domes were found, the background surface finish of the coating was smooth, as can be seen in Fig. 2-7. We determined that the coating was 10 weight \% (2 atomic

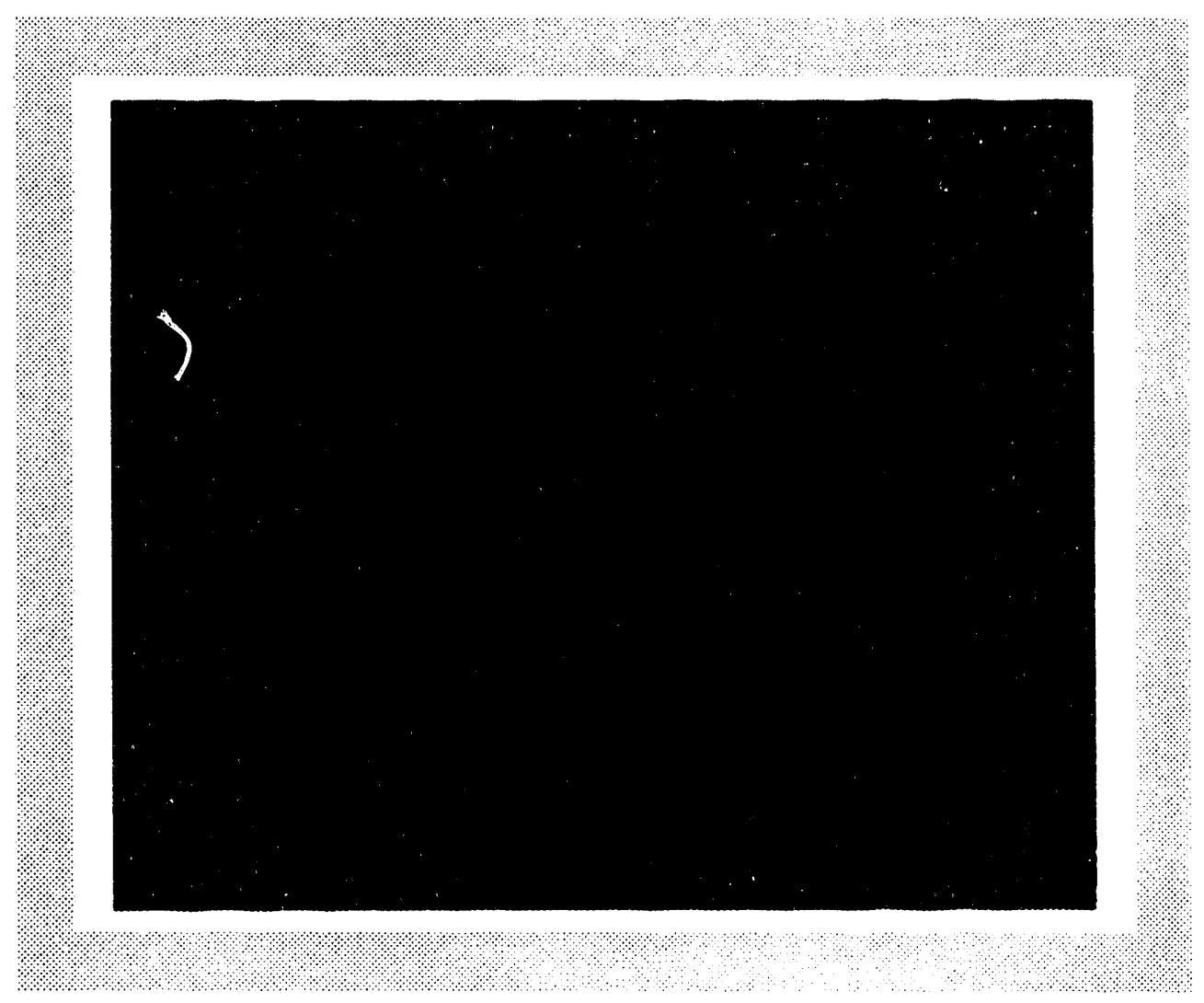

Fig. 2-7. SEM micrograph of germanium-doped $\mathrm{CH}$ coating deposited on a silicon wafer. 
weight \%) germanium by doing energy dispersive analysis of the characteristic $\mathrm{x}$-rays generated in an SEM and comparing the results with those obtained from a germanium sample.

For further information, please contact J. Ankney (GA).

\subsubsection{PARYLENE COATINGS}

In FY92, we assembled a parylene coating system for coating flat substrates [Ref. 2-4]. In FY93, we modified and upgraded the coater to allow us to coat ICF capsules. A schematic of the coater is shown in Fig. 2-8.

The following modifications were made to the basic system:

- For better temperature control of the sublimator, we installed a temperature controller.

- We removed the quartz wool from the pyrolyzer region. It was found to be a source of contamination due to fragments migrating to the coating region.

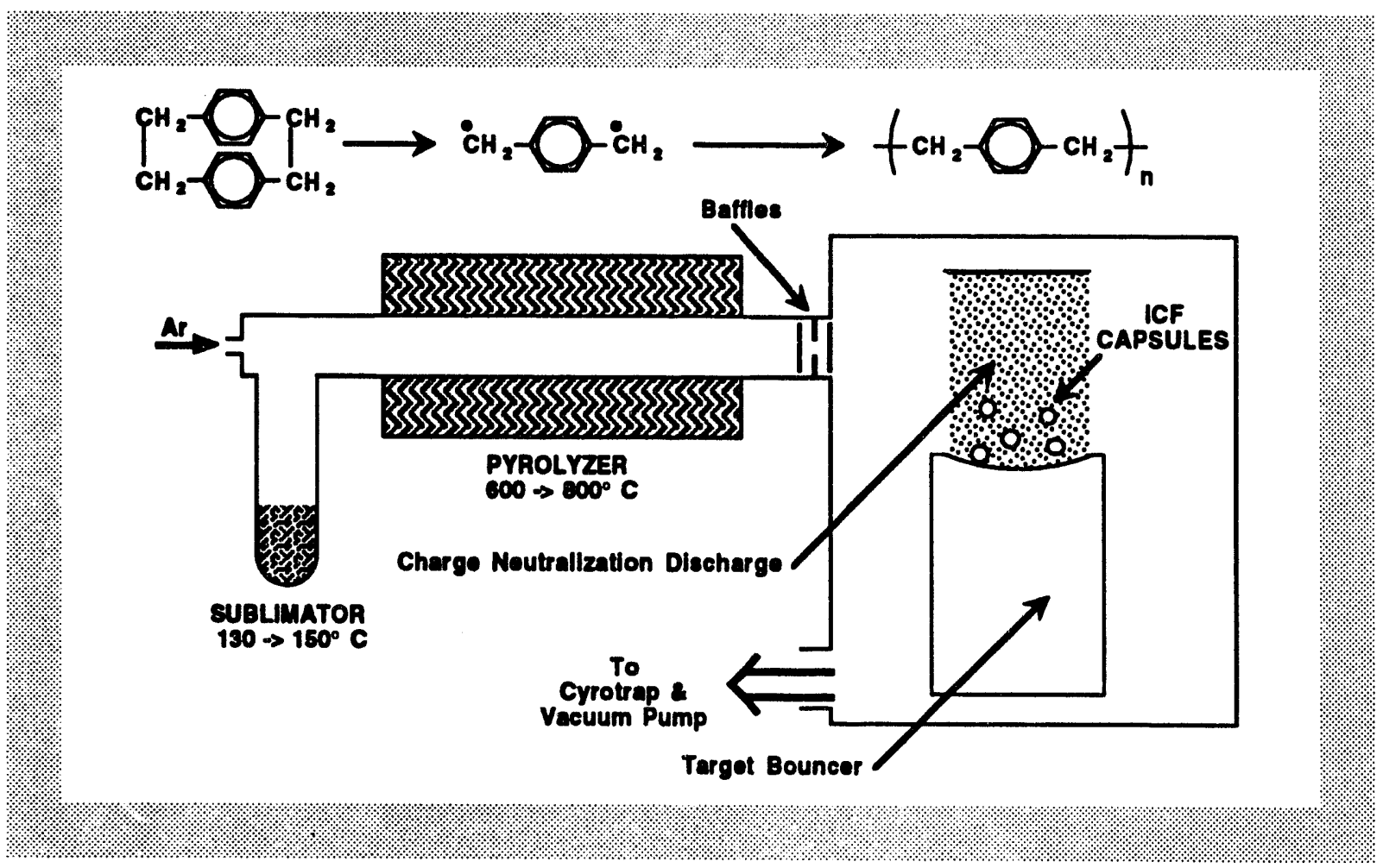

Fig. 2-8. Schematic of the Parylone Coating System. 
- To control particulates and decrease the temperature of the coating material, we installed a baffle system between the pyrolyzer and the coating region.

-We added a system to cool flat substrates or large samples during coating.

In addition to these modifications, we installed a bounce pan system given to us by the University of Rochester's Laboratory for Laser Energetics (UR/LLE). It uses a resonantly driven cylinder to bounce the target shells [Ref. 2-5]. To keep the shells from sticking, a glow discharge is generated over the bounce pan [Ref. 2-6]. The electrodes that create this discharge are designed to localize it in a column, as shown in Fig. 2-6. The discharge is created in argon introduced into this region and by an ac potential of $\sim 500 \mathrm{~Hz}$ with a peak-topeak voltage of $\sim 750$ volts between the plate electrode and the bounce pan.

Coating thickness is monitored in real time using the same method used by the UR/LLE [Ref. 2-7]. Specifically, a laser beam is reflected from a witness plate placed in the coating region onto a detector. As material is deposited on the plate, light reflected from the surface of the material and from the plate alternately interfere with and enhance each other. This allows determination of the thickness of the material deposited.

The capabilities that have been demonstrated using the coating system are summarized in Table 2-2.

For further information, please contact Dr. M. McClellan (WJSA).

\begin{tabular}{|c|c|c|}
\hline \multicolumn{3}{|c|}{$\begin{array}{c}\text { Table 2-2 } \\
\text { Capabumies of the Parkene Contwa System }\end{array}$} \\
\hline Capability & Range & Typical Value \\
\hline Coating rate & $0.5-5 \mu \mathrm{m} / \mathrm{hr}$ & $1 \mu \mathrm{m} / \mathrm{hr}$ \\
\hline Coating thickness & $0.6-40 \mu \mathrm{m}$ & $5 \mu \mathrm{m}$ \\
\hline Coating roughness & $30-2000 \AA$ & $200 \AA$ \\
\hline
\end{tabular}




\subsection{CHARACTERIZATION}

A key part of target fabrication is precise characterization. We have made several improvements in characterization techniques.

\subsubsection{OPTICAL TECHNIQUES - PVA CULL}

The goal of the composite shell production task is to efficiently produce shells with uniform walls of proper dimensions and surface finish. It is generally accepted that the wall uniformity of the final capsule is primarily determined by the uniformity of the polystyrene and PVA layers. Since the GDP coating process is very time consuming, it is important to ensure that only high quality PVA coated mandrels go into the GDP coating chamber.

We have instituted a three-step culling process which minimizes handling of the selected shells, retains statistical information about the defects on the rejected shells, and minimizes labor. It utilizes a zoom microscope, selected for its low distortion lens, suspended on a boom over a catch plate holder which allows complete inspection of 18 inch diameter catch plates (Fig. 2-9). The zoom and focus controls are motorized. The holder is sufficiently high to admit a microscope light base underneath. A CCD camera is mounted on the microscope; the operator views the shells on a monitor or, through a frame grabber, on a computer screen. The catch plate is illuminated at a glancing angle with a focused light beam to illuminate and highlight shells so that the operator can easily find them.

In the first step, each shell is picked off the plate and placed on a freshly cleaned glass block under the microscope. As the shell is set down, the operator examines it at low magnification (10-50X) for a hierarchy of possible defects (dents, bubbles, debris, etc.). If defect(s) are seen, the shell is discarded, and the highest level defect-type is incremented in a statistical table. The catch plate is rotated as its surface is culled of shells to bring new areas into the illuminated region for shell retrieval. This continues until the catch plate is cleaned of shells.

Shells surviving the cursory examination are lined up in rows on the glass block and examined by the zoom microscope at higher magnification $(100 \mathrm{X})$, in transmitted collimated light (not diffuse light). With this configuration, defects only scatter light out of (not into) the microscope's collection angle, and anomalous lenses or scatterers show up with strong contrast. This inspection reveals many of the smaller defects, such as small 


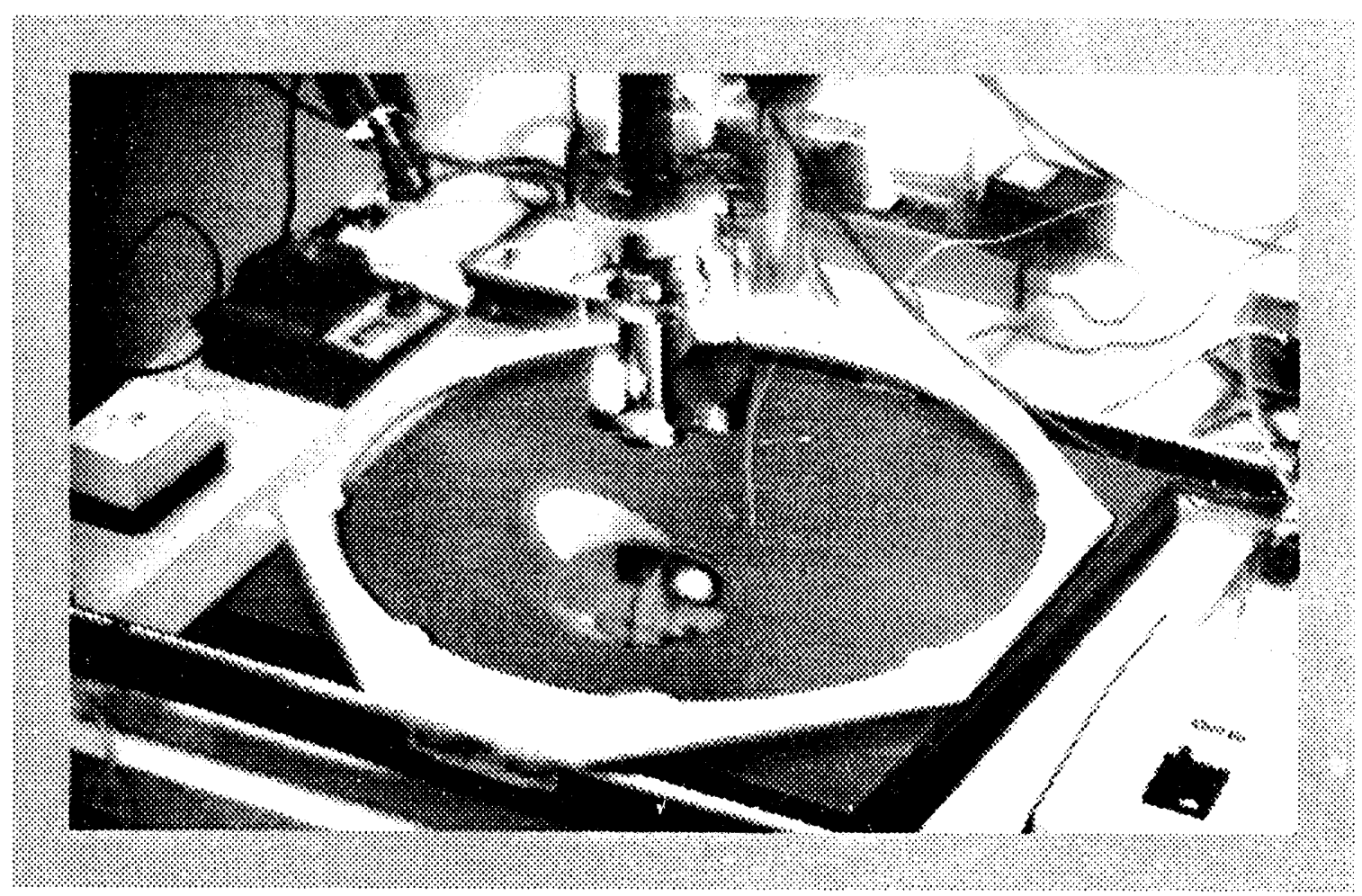

Fig. 2-9. PVA cull microscope work-station. The catch plate, an 18 inch diameter glass disk set in an aluminum frame, can be moved under the microscope to viow all sectlons; it rotates on its Teflon support pads, and slides on the rails. The $200 \mathrm{~m}$ microscope settings are controlled by the joy-stick on the right of the work-station. The sample is Illuminated by a fiber-optic source (in back) reflecting off a rotatable mirror in the base.

debris or PVA "dots" (see Fig. 2-10) that can result in mounds in the GDP coating. Again defective shells are discarded, and the defect-type tabulated. Our experience has been that a shell showing no defects under this examination will produce acceptable GDP coatings. Sub-micron scatterers and very weak lenses may be observed in dark field under the zoom microscope (the collimated beam is directed so that it is not collected by the microscope unless deflected by lenses or scatterers on the shell) but it is more efficient to do that in a third examination. Shells which are found to be essentially defect free are left in place on the glass block to minimize handling and loss of good shells. The rest of the shells are discarded or set aside as witness shells for characterization of their argon content, using XRF spectroscopy, and their surface finish, using the SEM.

In the final culling step, the remaining candidate shells are examined under a Jenavert interference microscope for submicron flaws, and for wall thickness and uniformity. This 
microscope can image smaller scatterers than the zoom microscope. In addition, interference fringes show long range wall thickness fluctuations, visible as fringe offsets or irregularities (see Fig. 2-11) which were not visible in the previous examinations, but which may allow argon or $D_{2}$ to leak through the PVA layer. Acceptable shells are sent on to the GDP coater, and defect information is added to the statistical table for the batch.

The defect information from each of these culling steps is entered into a premade Excel form (see Fig. 2-12). It lists the possible shell defects in an heirarchical order. The worst defects ("ugly" shells) are: shells stuck to the catch plate, strings of shells, mangled shells, large bubbles in the PVA coating, and gross debris. The less obvious defects ("bad" shells) are: dents, small bubbles, small debris, tool marks, and "other" (PVA flow, lenses, dewetting, etc.). This list is growing smaller over time as defects are eliminated. Each defective shell is tabulated only under the observed defect highest in this hierarchy (no matter how many types of defects it has). With this procedure, we know the number of shells examined for any given defect includes: the shells with that defect, shells with a defect lower in the hierarchy, and the good shells. Thus, we can calculate the statistical occurrence of each defect (assuming that they all occur independently), from the number of tabulations of a given defect divided by the number of shells which were examined for that defect.

For further information, please contact Dr. M. Hoppe (GA).

\subsubsection{X-RAY RADIOGRAPHY - IMAGE ANALYSIS}

$\mathrm{X}$-ray radiography is the most direct method of measuring the outer-, inner-, and interlayer dimensions of composite shells. Even in the simplest composite shells, one can see a layer contrast arising from the difference in $x$-ray absorption of the different plastics (PVA, containing oxygen, is substantially more absorbing than polystyrene or a GDP coating), as shown in Fig. 2-13. This layer contrast should create cusps in the radial profile of the film density at each edge. However, photographic grains on the $x$-ray plate add noise to radial profiles which can easily mask the real cusps.

We developed a radial averaging computer routine to eliminate the uncertainty caused by the film grains. The routine finds the center of a circular image, displays a selected segment of the radius in a rectangular plot of radius distance versus angle, and computes the radial intensity profile averaged over all angles. This routine has been added as a menu command to Image, a "freeware" image analysis program from the National Institute of 
GA-A21647 FY93 ICF Annual Report

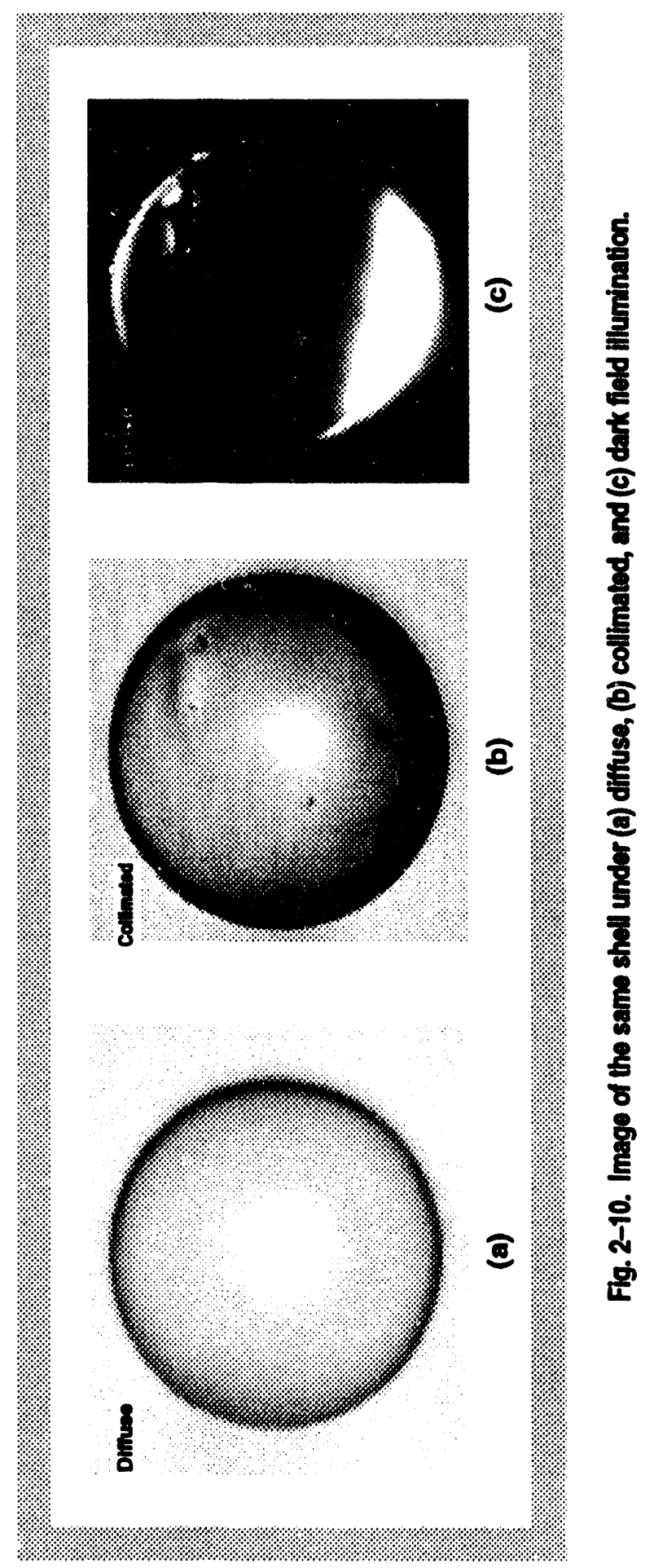

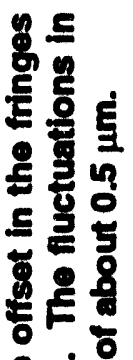

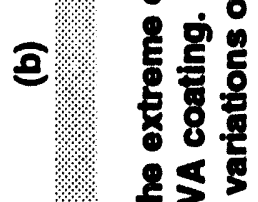

Fล:

8 \&

동호

훙

흥

톨돌

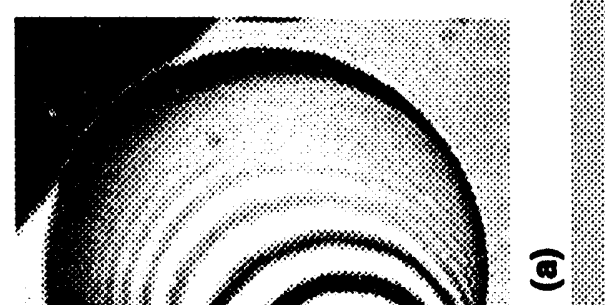

$5 \geq \frac{9}{1}$

올

ร융

용

कु

으롱

혼

ธ옵 능

8으믈

트용

完 


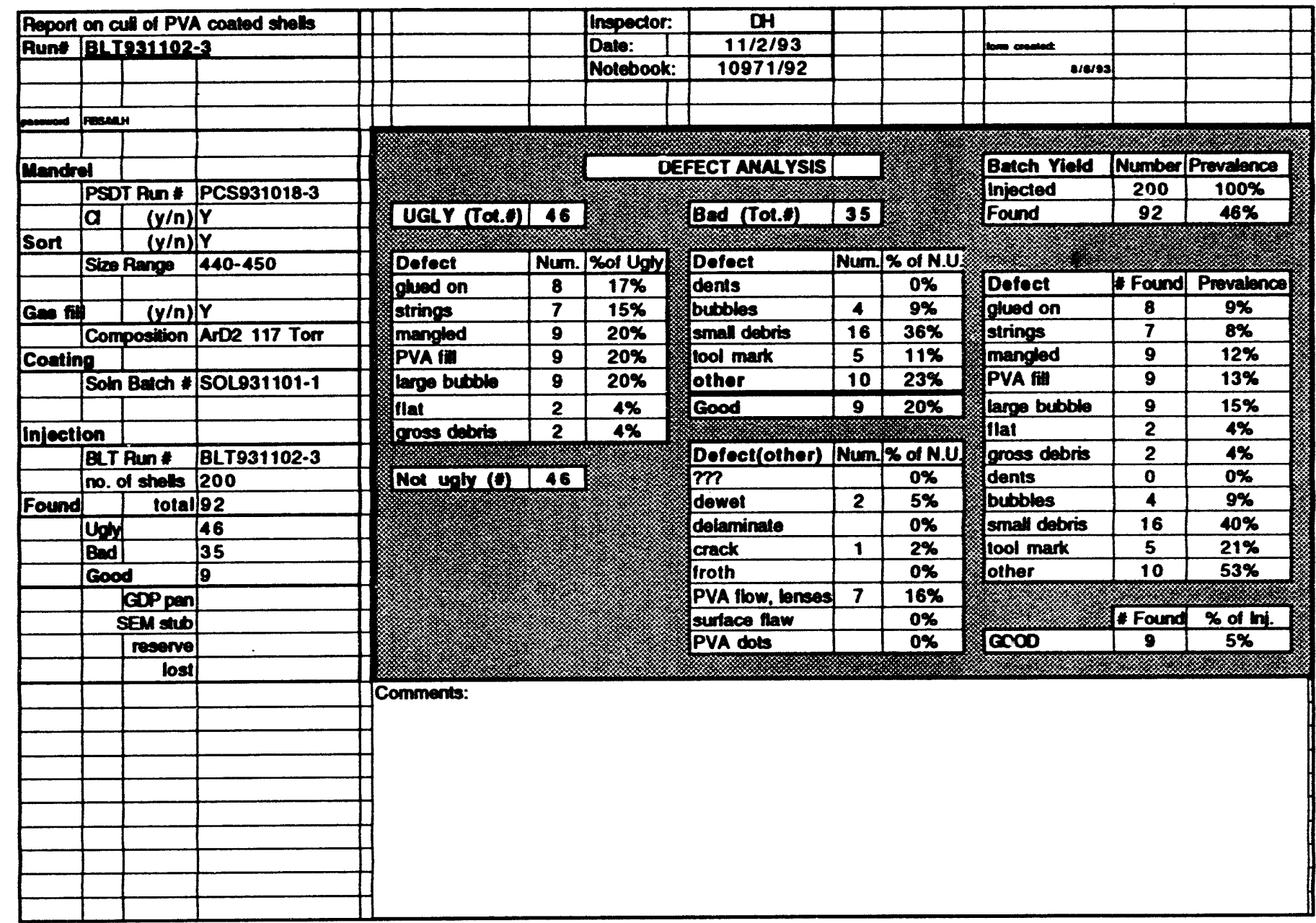

Fig. 2-12 PVA cull statistics worksheet. It shows batch production information on the left side and summarizes defect statistics on the right. 


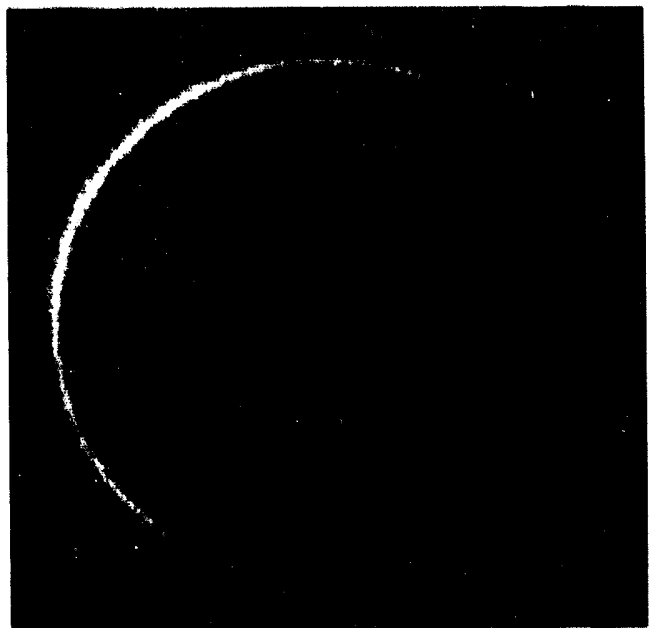

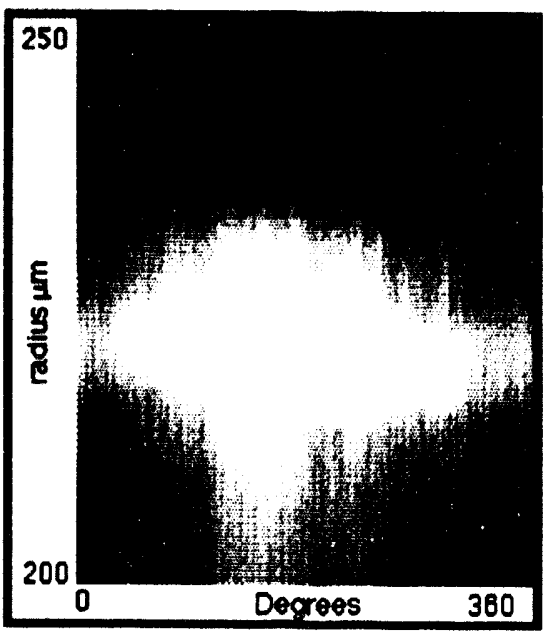

(b) (a)

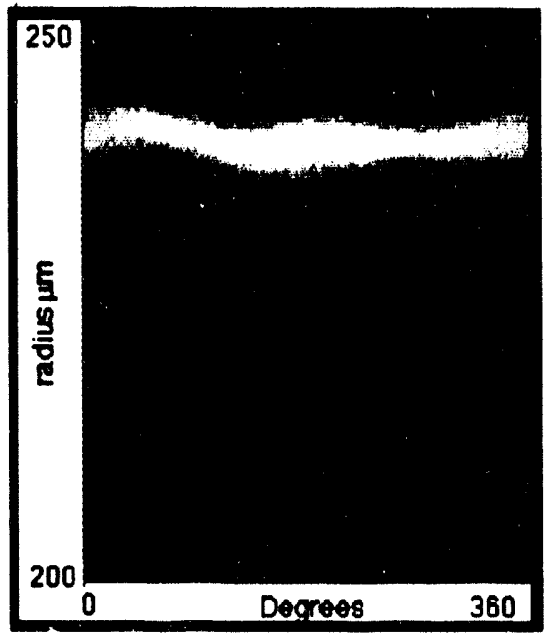

(c)

Fig. 2-13. (a) X-ray radlograph Image of composite sholl using 5 koV radiation; (b) rectangular plot of the edge of the shell shown in (a); (c) radial gradient modification of the plot shown in (b).

Health. We will release the modified Image program for general use after some final polishing and checking.

The intent of the routine is to more accurately determine edge and cusp positions by averaging the radial intensity profile over many radii. The radiograph is imaged under a microscope, and a file created containing the complete image of the shell. The computer file is opened in the revised Image. A user indicates the Region Of Interest (ROI) by 
selecting a rectangle whose edges touch the outside of the shell image and starts the center finding routine. The module calculates the average radial intensity profile for a set of trial centers. The trial centers are changed until the maximum contrast of the radial average profile, indicative of the shell center, is found. This routine finds the center to sub-pixel accuracy.

The next step is to collect a final set of radial intensity information (it did so in the first step, also). The user specifies the range of radial distances, and the radial and angular spacing for which information is to be collected. When the radial average routine is started, the program finds the location of each point on each radius relative to the screen pixels, and calculates its intensity by a weighted averaging of the intensity of the four nearest screen pixels.

A plot is made of each radius range versus the radius angle in a new window [see Fig. 2-13(b)], and the $360^{\circ}$-averaged radial intensity is put into a new data file. The plot of radial intensity versus radial angle may be modified with the other tools in Image to enhance visibility of the edge. The plot in Fig. 2-13(c) is the convolution of Fig. 2-13(b) with a radial gradient plus the original picture. The inside edge and the PVA-GDP interface, which in the model occur at points of steepest slope in the intensity, are visible as the inside edges of the dark and light bands, respectively, produced by the gradient convolution.

The file containing the $360^{\circ}$-averaged radial intensity profile can be inserted into an Excel spreadsheet and compared to model calculations. Figure 2-14 shows the average profile (smooth curves) compared to a model calculation (cusped curves). The experimental curve is smoothed by the noncircularity of the image and by the pixel resolution of the CCD camera [about $2 \mu \mathrm{m}$ in this case as indicated by the rounding at the outside edge of the profile in Fig. 2-14(a)]. The relation between film absorption and plastic thickness is non-linear because of the wide spread in absorption lengths of the x-ray spectrum with which the plastic was irradiated; the better fit took that nonlinearity into account, while the other simulated profile assumed a linear relationship between plastic thickness and film density.

For further information, please contact Dr. R. Stephens (GA).

\subsubsection{INDEX OF REFRACTION MEASUREMENTS - IN SITU}

Thickness measurements by interference microscopy requires knowledge of the index of refraction, $n$, of the layers being measured. $n$ changes significantly with wavelength, increasing 

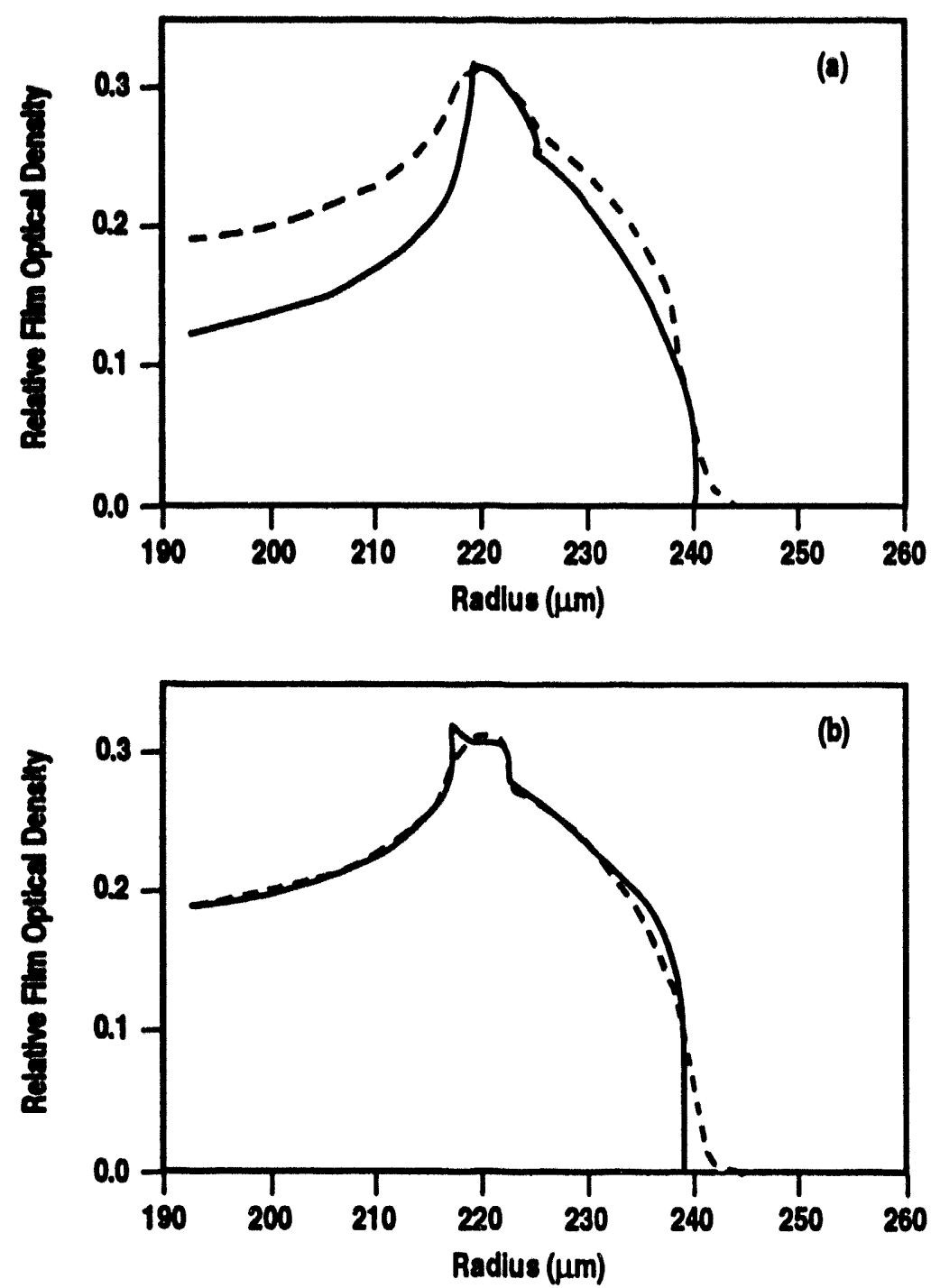

Fig. 2-14. Radial film density profile for the shell shown in Fig. 2-15 compared to two simulated curves. The model in (a) assumes a linear relationship botween plastic thickness and film optical density. The model in (b) assumes a nonlinear relationship.

at an increasingly rapid rate toward the blue end of the spectrum. The proper value of $n$ is a convolution of its spectral dependence with the illumination spectrum. It sometimes depends, in addition, on preparation procedures. For instance, the index of refraction of GDP coatings depends strongly on plasma power, gas composition, etc. As a result, we could not depend on literature values of $\boldsymbol{n}$ for the polymers in our composite shells. 
The procedure we developed to determine shell material $n$ involves matching the shell material with a calibrated index-matching oil. The sample is immersed in the index matching oil, as shown in Fig. 2-15, and an interference microscope compares the optical-path-length through the oil, and through an oil/sample combination. Because $n$ of the oil and the sample are the same, there are no refraction effects, and one can use any sample large enough to see through. Figure 2-16(a) shows a match for a $50 \mu \mathrm{m}$ thick GDP coating on a shell. This match can determine $n$ of the material to $<0.002$. The index of refraction of shell layers less than $10 \mu \mathrm{m}$ thick cannot be measured by this technique due to magnification limitations. To determine $n$ of PVA, we measure PVA witness strips as shown in Fig. 2-16(c).

Getting the accurate match shown above involves a considerable number of trials. A very good approximation can be made by interpolating between the fringe offsets seen using only two index matching fluids. Using an interference microscope set up as shown in Fig. 2-16, we measure the fringe shift, $f_{j}$, of the light passing through the sample versus light passing adjacent to the sample through two index matching fluids having $n_{i}$ of $n_{1}$ and $n_{2}$ respectively. The fringe offsets $\left(f_{1}\right.$ and $f_{2}$ ) in each case are the product of the average wavelength, the sample thickness, and the difference in index of refraction, $\left(n_{\text {mat }}-n_{1}\right)$. Solving for $n_{\text {mex }}$ the sample thickness and average wavelength cancels out, to give:

$$
\mathrm{n}_{\text {mat }}=\frac{\mathrm{f}_{2} \mathrm{n}_{1}-\mathrm{f}_{1} \mathrm{n}_{2}}{\mathrm{f}_{2}-\mathrm{f}_{1}}
$$

The result obtained using this method can be confirmed by placing the sample in index matching fluid of the derived index of refraction.

For further information, please contact D. Steinman (GA).

\subsection{GAS FILL SYSTEM}

We designed and built a system for filling polymer and glass shells with up to 400 atm $(6,000 \mathrm{psi})$ of deuterium at $360^{\circ} \mathrm{C}$. This is equivalent to a 200 atmosphere fill at room temperature.

Target shells are filled with gases through the permeation process. Gas permeates through the shell wall at a rate dependent on wall thickness, the difference in gas pressure between the two sides of the wall, and the permeation constant. In general, the permeation 


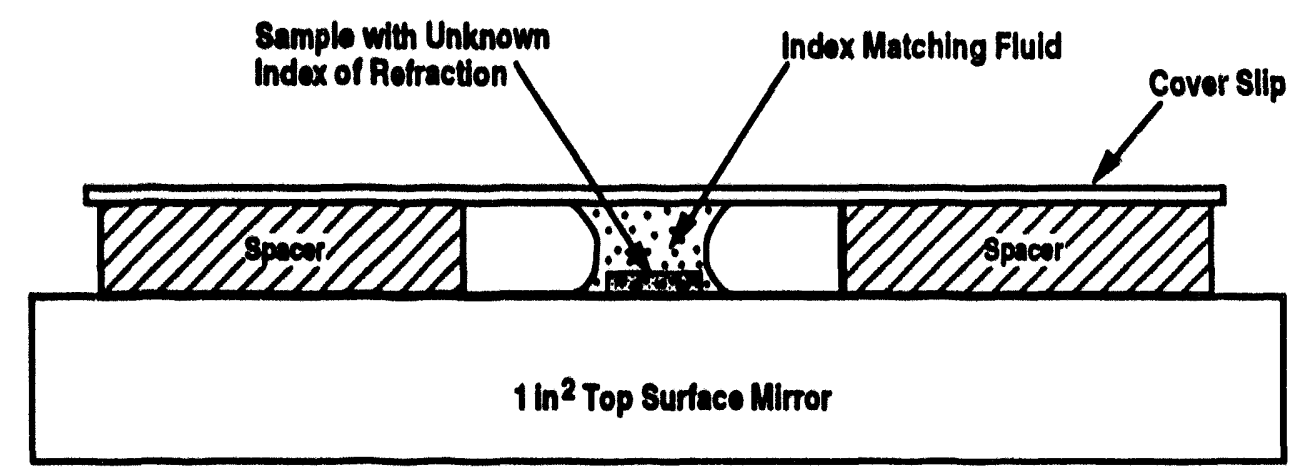

Fig. 2-15. Side viow of apparatus used for dotermining index of refraction of sample material. The cover sllp provides a flat, distortion-free window for looking into the oll drop. The substrate could be elther reflective or transparent, to sult the requirements of the interforence microscope.

constant increases with temperature. We permeate gas into glass shells at about $360^{\circ} \mathrm{C}$ to obtain equilibrium conditions in a reasonable time; at room temperature, permeation is several magnitudes slower. Polymer shells require only slightly elevated temperatures, about $50^{\circ} \mathrm{C}$, for acceptable rates of permeation.

Target shells can be destroyed by two processes occurring during the fill. When the pressure outside the shell is considerably greater than the pressure inside the shell, the shell will buckle. If the pressure inside the shell is considerably greater than the pressure outside the shell, the shell will explode. To minimize shell destruction via buckling we take two precautionary actions. First, we only fill shells that have survived pressures slightly larger than the pressure step used during the fill. Second, we move to the final fill pressure in small steps (to minimize shell breakage) held long enough for permeation to increase the pressure inside the shell. We minimize explosive shell destruction by selecting shells likely to withstand the inner pressure desired.

\subsubsection{SYSTEM DESCRIPTION}

The GA fill station is shown in Fig. 2-17. It was constructed with stainless steel tubing and fittings from Swagelock, VCR, and HIP. The permeation cell is a 1 inch diameter tube 


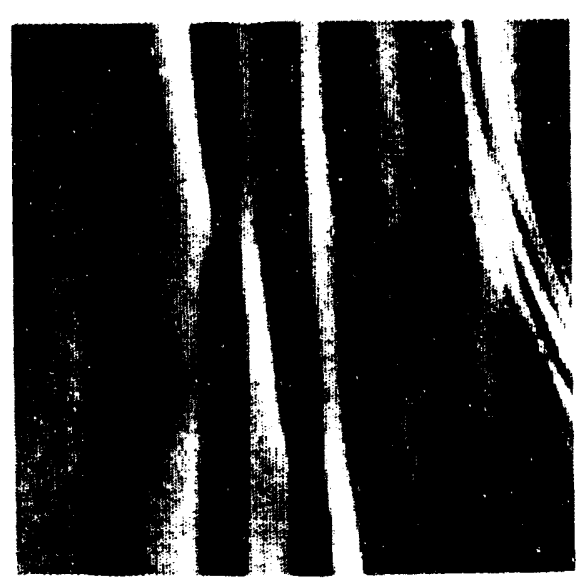

(a)

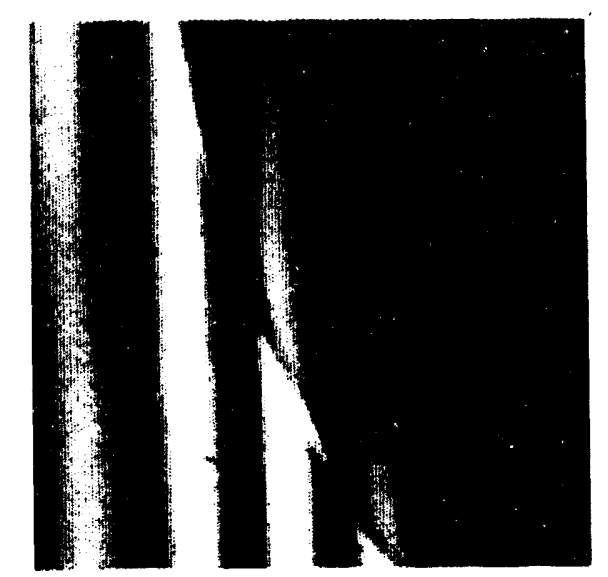

(b)

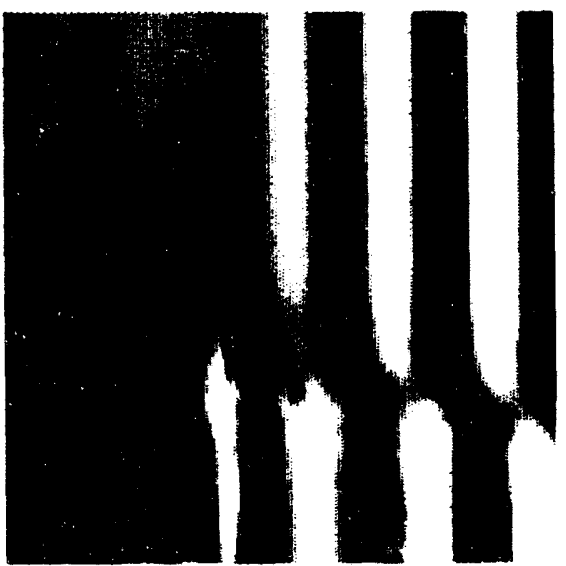

(c)

Fig. 2-16. White light Interforence micrographs of objects in Indox matching solution. (a) and (b) are of the GDP coating of a composite shell. The fluid is matched in (b) and mismatched in (a) by $\Delta n=0.002$, causing the Interference lines to shift approximatoly 1/2 fringe spacing (c) is of a PVA film deposited on glass.

that contains an aluminum "egg crate" filled with shells. A Lindberg furnace surrounds the permeation cell and a vacuum pump evacuates the system. The system is pressurized with deuterium from a gas bottle. At pressures below 1,000 psi (70 atm), the permeation cell is filled directly from the gas cylinder. For higher pressures, a Haskel booster pump is used to increase the pressure up to $6,000 \mathrm{psi}(400 \mathrm{~atm})$. The temperature and pressure of the permeation cell are continually monitored. After allowing sufficient time for permeation, the pressure in the permeation cell can be increased. When permeation is complete, the cell is cooled and the egg crate holding the shells is removed. 


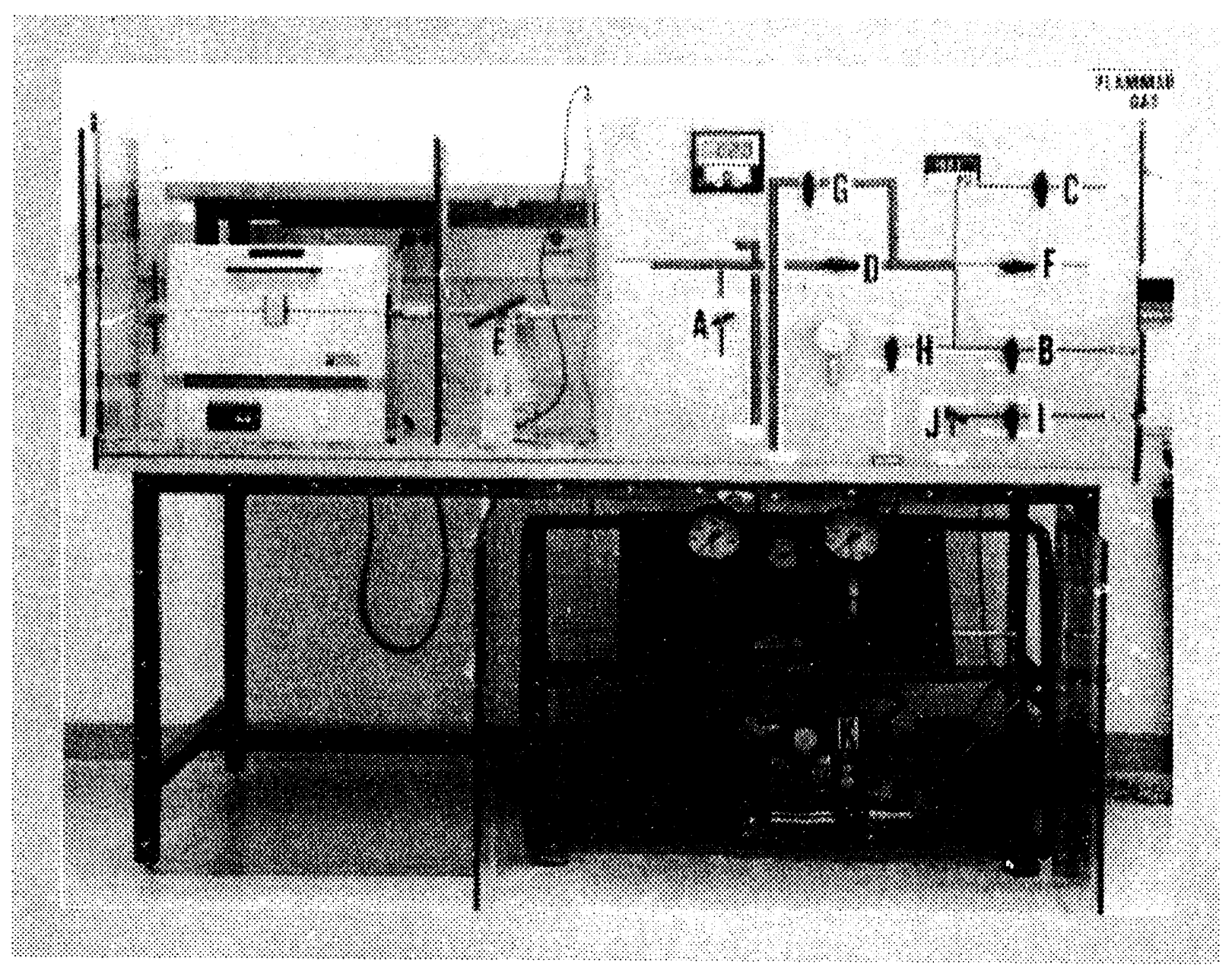

Fig. 2-17. Deuterium fill system.

\subsubsection{GLASS SHELL FILL TEST}

After assembling the system, we successfully leak checked the system at low pressure, using a helium leak detector, and at high pressure by observing the change of pressure with time. In FY93, we completed a test fill of glass shells to a nominal pressure of $100 \mathrm{~atm}$. The shells were tested for fill pressure by interferometry and by the Gas Entrapment in Viscous Fluid (GEVF) technique [Ref. 2-8]. Both pressure measurement techniques were new to GA; these glass shells were our first high pressure use of the techniques. The results from the tests indicated that the shells had filled to a pressure of 96 atmospheres, in good agreement with our expected pressure.

For further information, please contact W. Miller (GA). 


\subsection{MICROMACHINING}

An important part of ICF target fabrication is micromachining target components. Our micromachining capability became production-ready this year.

\subsubsection{MANDRELS}

The overall goal of the Micromachined Target Component Facility task was to acquire the capability to produce micromachined target components. With assistance from LANL personnel, we identified a diamond turning machine manufactured by the Precitech Company of Keen, New Hampshire, as very suitable for our application. We ordered this machine, and it was received in July, 1993. In addition, we ordered and received a laser micrometer and a machinist's microscope for measuring the dimensions of machined components. A fourth necessary piece of equipment, a precision indicator, was received on loan from LANL.

Since the room originally assigned for micromachining in the GA Target Fabrication Facility was too small to house the Precitech lathe and the diamond turning machine that we expect to receive from the DOE Rocky Flats Plant, an additional room was constructed within the coatings laboratory to house the micromachining equipment. This room has its own separate air conditioning system to help maintain the micromachining equipment at a constant temperature. In addition, the Precitech lathe has its own "air shower" which continually bathes the lathe in constant-temperature air.

GA and WJSA personnel received training in the operation of the micromachining equipment during extended visits at Precitech and at LANL.

A drawing of the mandrel suggested by LLNL for use in our capability validation tests of the micromachining facility is shown in Fig. 2-18. This mandrel has many challenging features for the machinist, including: large radii, tapers, small radii, a flat region, and very tight dimensional tolerances. After a period of learning to use the machine on our own, which no amount of training by others can replace, we produced a set of mandrels that met the specifications shown in Fig. 2-18. A scanning electron micrograph of one of these mandrels is shown in Fig. 2-19. 


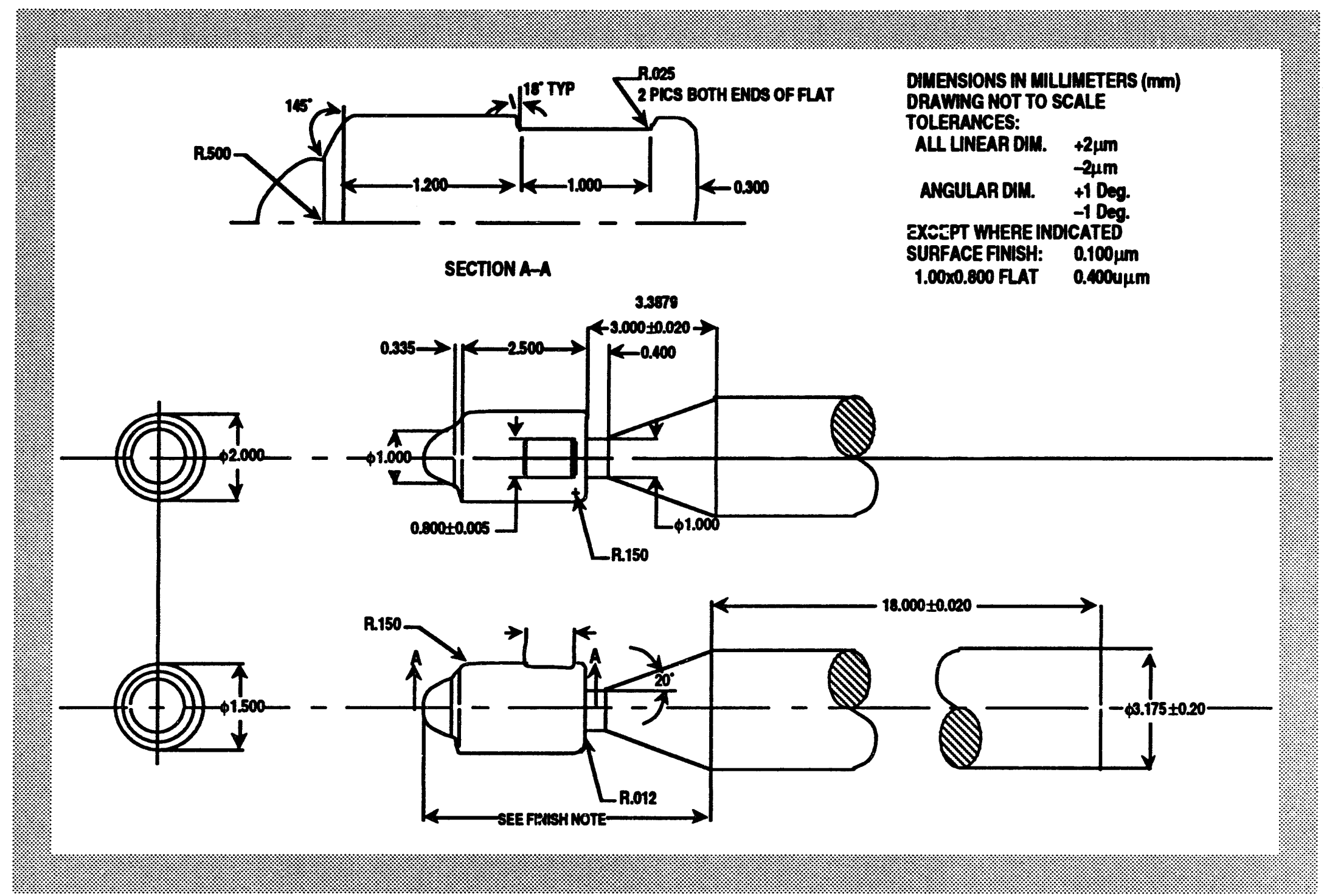

Fig. 2-18 A drawing of the test mandrel suggested by LLNL for capability validation testing of the micromachining facility. 


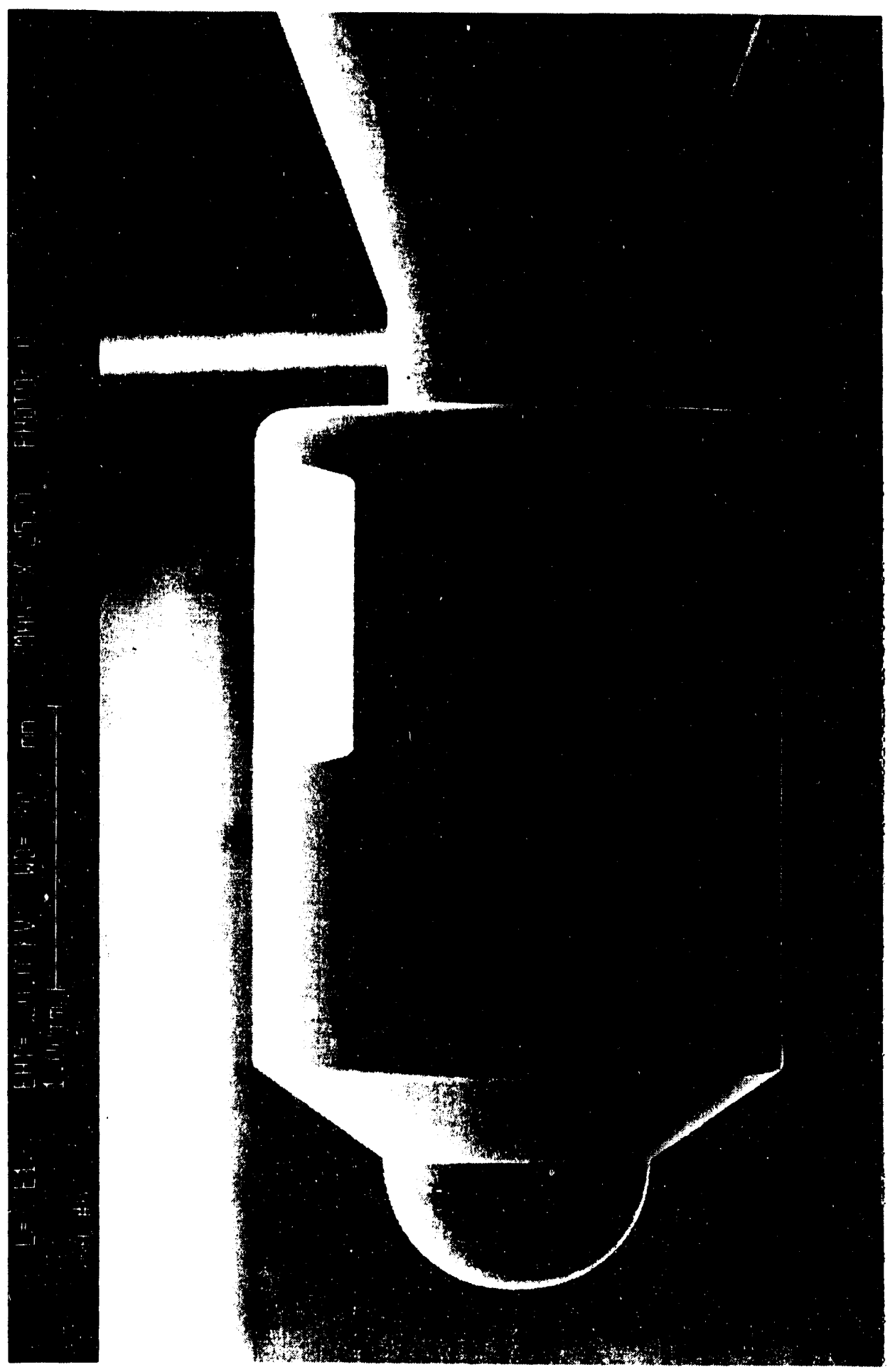

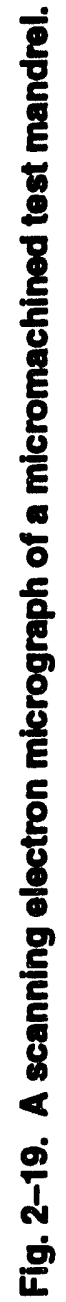


As indicated in Fig. 2-18, the smoothness of the machined surfaced is an important quality in micromachined target components. A typical roughness measured by a surface profilometer on the barrel of one of the test mandrels manufactured on the Precitech lathe is shown in Fig. 2-20. This surface has an average roughness of $215 \AA$ and far exceeds that required on the test mandrels.

The set of test mandrels produced on the Precitech lathe was shipped to LLNL for capability validation in September of this year.

For further information, please contact Dr. J. Kaae (GA).

\subsubsection{COATINGS}

In setting up a target component electroplating facility, we decided the best way to proceed would be to copy the successful target component electroplating facility at the

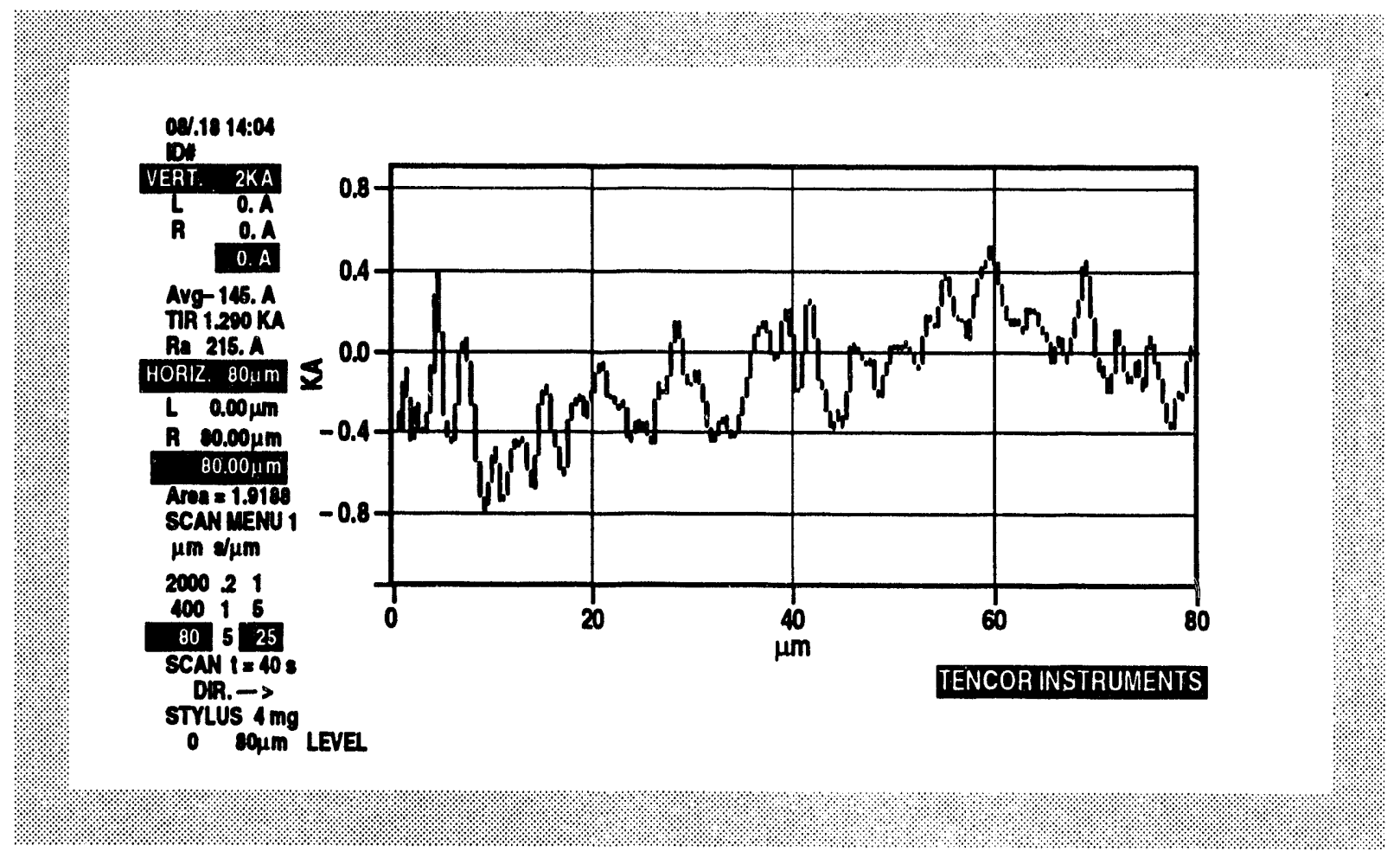

Fig. 2-20. A profilometer trace of the barrel surface of a test mandrel. The average peak-tovalley roughness is $214 \mathrm{~A}$. 
Rocky Flats Plant as closely as possible. To this end, several visits were made to observe the equipment and procedures used at Rocky Flats.

The room previously set aside for micromachining in the GA ICF Target Fabrication Facility was selected for the Target Component Electroplating Facility.

Necessary equipment identified in the visits to Rocky Flats was purchased or fabricated. Included was a copy of the twelve-specimen rotator used to plate twelve specimens at a time at Rocky Flats as shown in Fig. 2-21.

A set of rough-machined copper mandrels was used for tests of our plating techniques during training of our operators. Once we had worked out the techniques, a set of reject mandrels machined at Rocky Flats was used for plating tests. Plated specimens were examined with the SEM and thicknesses were accurately measured with a laser micrometer. In the course of developing techniques for plating, several types of defects were found and eliminated. These defects were of the following types:

1. Circular defects produced by residue of cleaning solutions on the surface.

2. Non-uniform plating produced by current flow from the wire leading to the circular anode.

3. White stains produced by incorrect post-plating cleaning.

4. Organic contamination produced by contamination of the plating solution by unknown sources.

5. Large grains produced by a low level of brightener in the plating solution.

Several Rocky Flats-machined, reject mandrels were plated and sent to LLNL for validation of our plating process. Shortly thereafter, we were given our first production order for plating of specimens. 


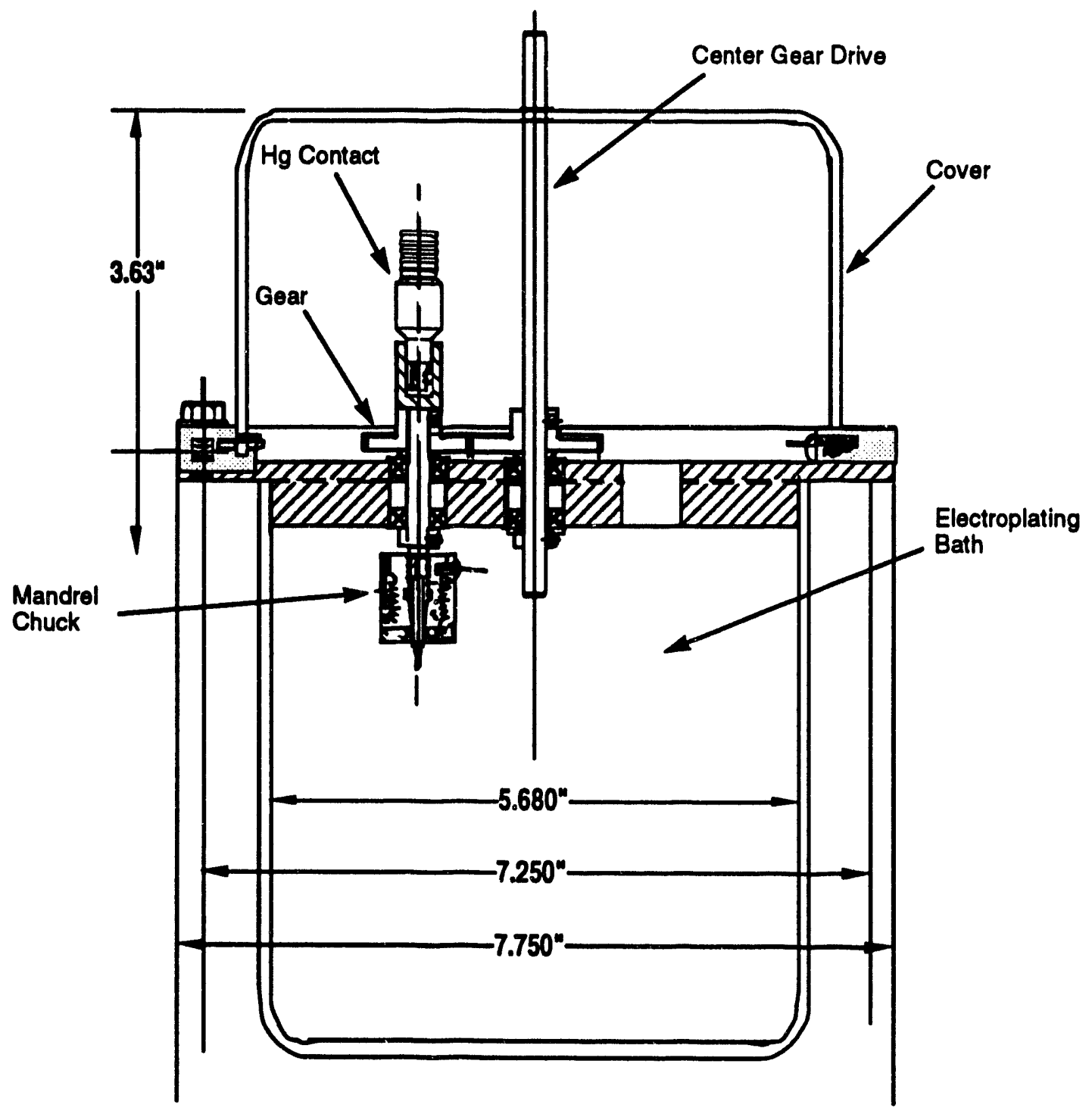

Fig. 2-21. A cross-sectional drawing of the specimen rotator. Only one of the 12 rotators is shown in place. 


\subsection{REFERENCES}

[2-1] ICF Target Fabrication Monthly Report for January 1993, General Atomics, San Diego, CA, ICFT93/033, (February 2, 1993) 4.

[2-2] Brusasco, R.M. and Ferguson, S.W., "Fundamental Studies of Helical Resonators Used in Plasma Polymerization for Target Fabrication," 1992 ICF Annual Report, Lawrence Livermore National Laboratory, Report UCRL-LR-105820-92, (June, 1993) 99.

[2-3] Brusasco, R.M., Status Update on Helical Resonator Coater Studies, Lawrence Livermore National Laboratory, Report TST 92-029, (February 7, 1992).

[2-4] McClellan, M.R., "Parylene Coatings," Inertial Confinement Fusion Target Component Fabrication and Technology Development Support, 1992 Annual Report GA-A21203, General Atomics, (1992) 2-17.

[2-5] Gram, R.Q., Kim, H., Mason, J.F., and Wittman, M., J. Vac. Sci. Technol. A4 (1986) 1145.

[2-6] Gram, R.Q., Immesoete, C.K., Kim, H., and Forsley, L., J. Vac. Sci. Technol. A6 (1988) 2998.

[2-7] Kim, H., Powers, T., and Mason, J., J. Vac. Sci. Technol., 21 (1982), 900.

[2-8] Rensel, W.B., Henderson, T.M., and Solomon, D.E., Rev. Sci. Instrum. 46(6), (1975) 787. 


\section{LABORATORY RESEARCH SUPPORT}




\section{LABORATORY RESEARCH SUPPORT}

An important part of our activities is to provide technical and scientific support at the ICF laboratories.

\subsection{CRYOGENIC TRITIUM RESEARCH AT LANL}

John Simpson of GA performed onsite cryogenic tritium research activities at LANL.

\subsubsection{INTRODUCTION}

Cryogenics experiments at LANL in FY93 have focused on the examination of DT solid layers inside a copper cylinder having a $2 \mathrm{~mm}$ inner-diameter, and the characterization of these layers for surface roughness. The DT solid layers are formed by a process known as Beta Layering, which has been described extensively elsewhere [Refs. 3-1 through 3-3] and has been shown highly effective at producing uniform and smooth solid layers [Refs. 3-4, 3-5]. Such layers are required for future ICF targets to achieve conditions necessary for ignition. The surface quality that we have been able to produce to date is still tco rough for the proposed National Ignition Facility (NIF) target [Ref. 3-6]. We have therefore continued to explore techniques for improving layer image quality, as well as the quality of the actual DT solid layer. This has included improving image analysis software [Ref. 3-7], image quality with flash illumination and cryostat/image synchronizing, and layer quality by warming the DT solid to temperatures near the triple point.

During previous experiments [Refs. 3-5, 3-8], we produced a $73 \mu \mathrm{m}$ DT solid layer with a surface roughness of just over $3 \mu \mathrm{m}$ ms, which included a sizable $(0.8 \mu \mathrm{m}) \mathrm{P} 1$ defect. The equilibration time constant for this experiment was unexpectedly slow (e.g., several hours), possibly showing the effect of the cylinder re-entrant geometry and/or a possible deuterium rich fuel mix. These results indicated that the process had not yet come to equilibrium when we terminated the experiment. Thus, our next task was to extend the experimental run far enough for equilibrium to be reached. We were also interested in improving the CCD image quality by using a pulsed illumination system and by synchronizing image acquisition to 
cryostat motion cycle. Furthermore, we attempted to improve layer quality by raising the DT solid temperature to near the triple point.

\subsubsection{EXPERIMENTAL SET-UP}

The configuration of the experimental apparatus, imaging system and synchronizing electronics, is shown schematically in Fig. 3-1. All the components for this experiment, except the Macintosh computer and electronics for the Photometrics CCD camera and synchronizing electronics, are contained in a specially designed glove box that functions as a hood. This allows us reasonable access to the internal apparatus when needed, and provides a fourth level of tritium containment in case of failure of the primary containment of the DT cell.

The DT cell has an inner diameter of $2 \mathrm{~mm}$, an inner bore $2 \mathrm{~mm}$ in length, and a counterbore $3 \mathrm{~mm}$ in diameter by $1 \mathrm{~mm}$ deep at each end of the primary cylinder. This re-entrant design gives us a clear view of the DT solid edge, unobscured by the DT solid wrapped around from the cylinder to the sapphire window. The cell is made of copper and is sealed with sapphire windows and indium O-rings, providing a tightly sealed isothermal container for the DT charge.

The optical system for this experiment uses a Questar (Model QM100) long range microscope coupled to a Photometrics (Model CE200A) CCD camera system, interfaced to a Mac Ifx. This combination provides the equivalent of about an $\mathrm{f} / 4$ optical system with the cell image nearly filling the $1024 \times 1024$ pixel CCD array, giving a spatial resolution of $2.5 \mu \mathrm{m}$ per pixel. Using analysis software provide by Mapoles of LLNL, we are able to measure solid layer uniformity to sub-pixel resolution. The latest version includes a non-linear fitting algorithm that improves our image resolution (reduces noise floor) by almost an order of magnitude. With this new software we are able to see DT surface changes approaching 100 angstroms in size.

Illumination for the cell is provided by a White Lightning Ultra (Model 1200) flash unit sold by Paul C. Buff, Inc., Nashville, TN. The pulsed illumination system is synchronized to the CCD camera shutter opening, and allows us an effective exposure time of $2 \mathrm{msec}$. This high intensity (26,000 lumen-sec) short duration light source, produces images with high contrast and less edge smearing from cryostat motion than the images produced previously with the $\mathbf{C W}$ incandescent source. This feature was only recently integrated into our system. It was in place for the $139 \mu \mathrm{m}$ layer experiment just completed. 


\section{DT Layering Experimental Set-Up at LANL}

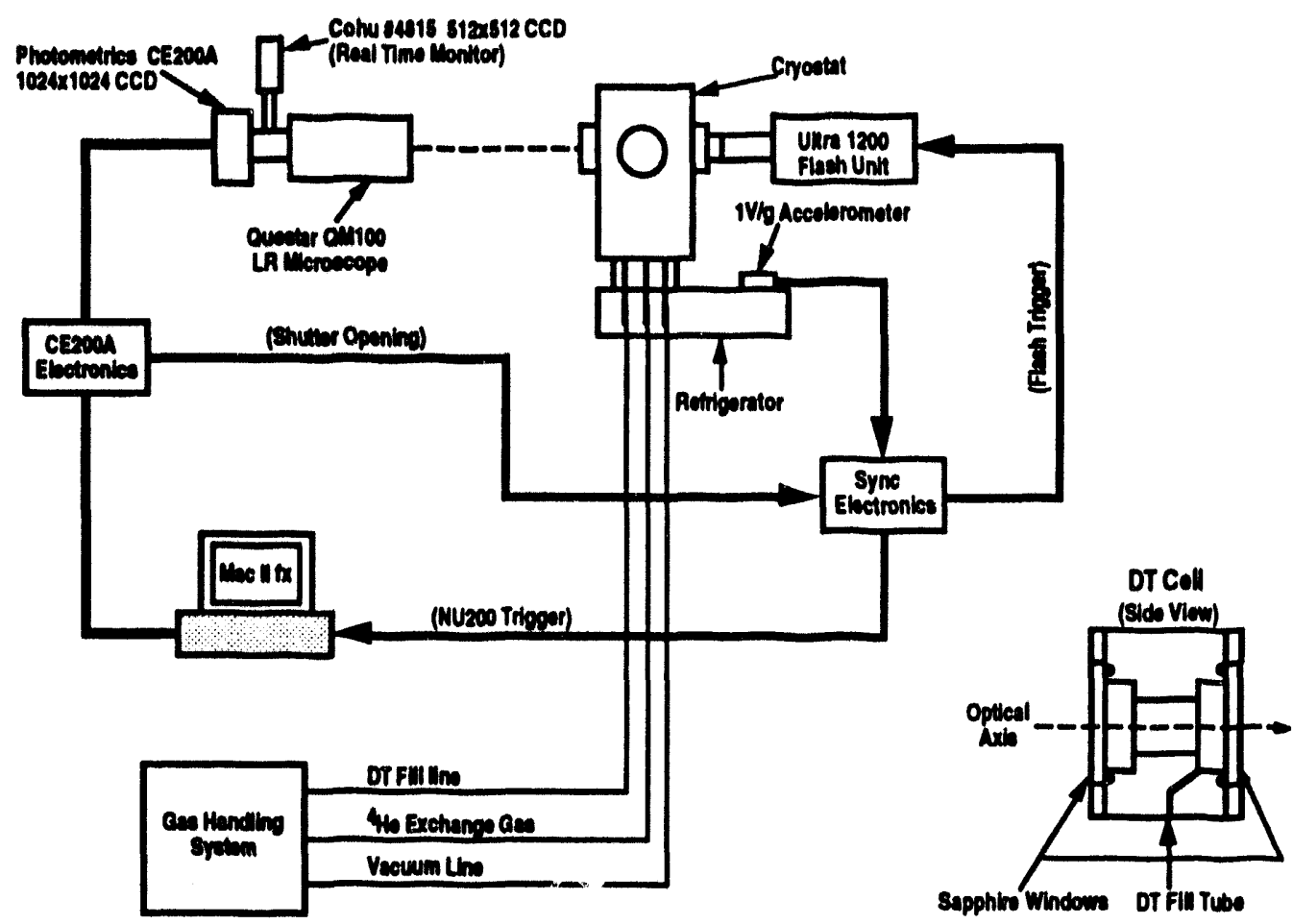

Fig 3-1. Schematic of high resolution optical DT layering experiment at LANL.

The final addition to our experimental set-up was the system used to synchronize the CCD image acquisition to cryostat motion using the output signal from a $1 \mathrm{v} / \mathrm{g}$ accelerometer mounted on the refrigerator, as shown in Fig. 3-1. This system consists of an accelerometer, signal processing and triggering electronics, and timing electronics to establish the image acquisition cycles. We designed the electronics to enable us to select a point in the cryostat cycle for which we would trigger our CCD camera exposure, then trigger the camera at the same point in the motion cycle each time. Using this system we were able to improve image quality by reducing image to image focal shifts, reducing scatter in the DT surface power spectra modes, and dramatically reducing shift in the image centroid (the center of the cell image). Figures 3-2 and 3-3 show the improvement in image acquisition obtained by synchronizing image acquisition to cryostat motion. 

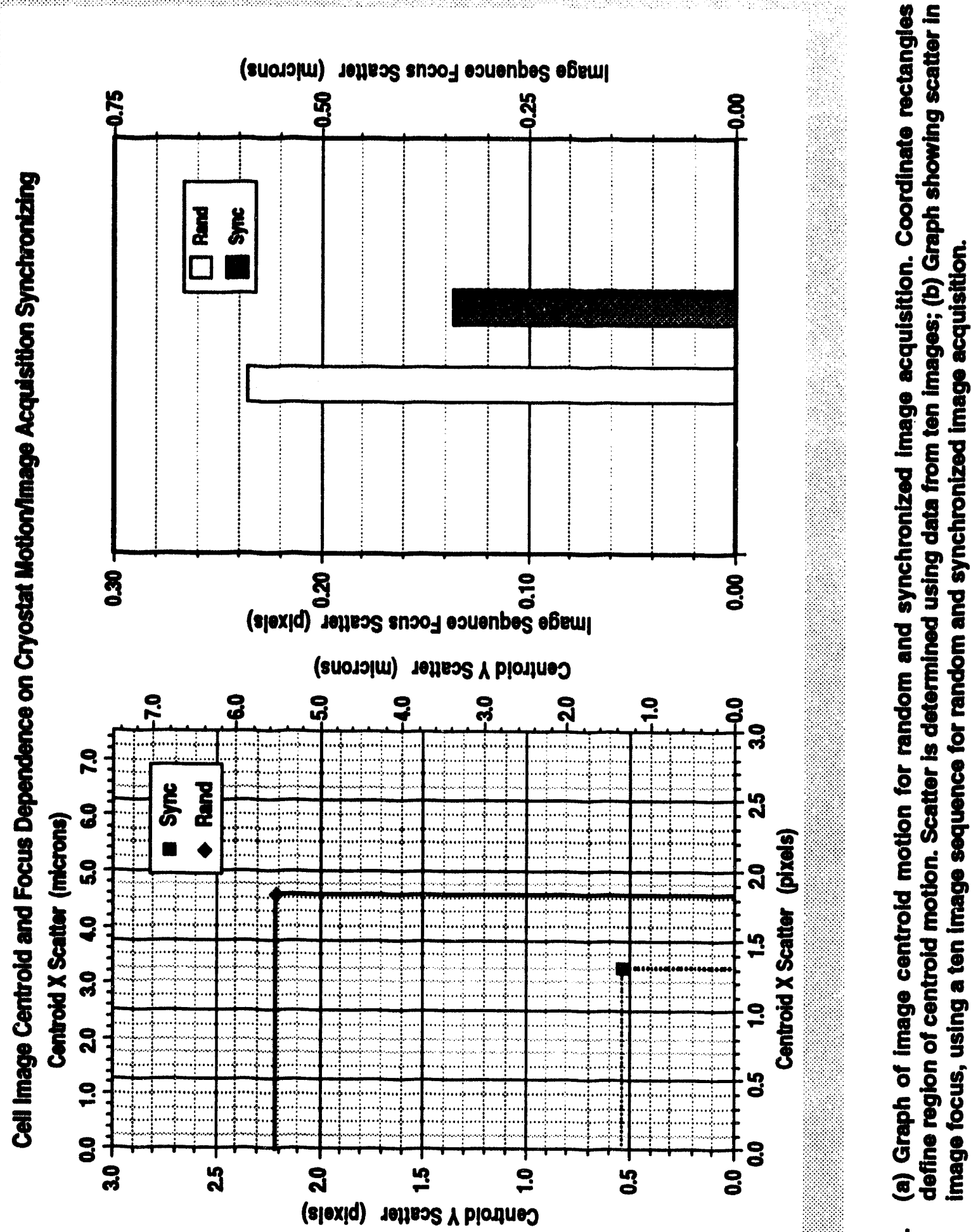

సे 


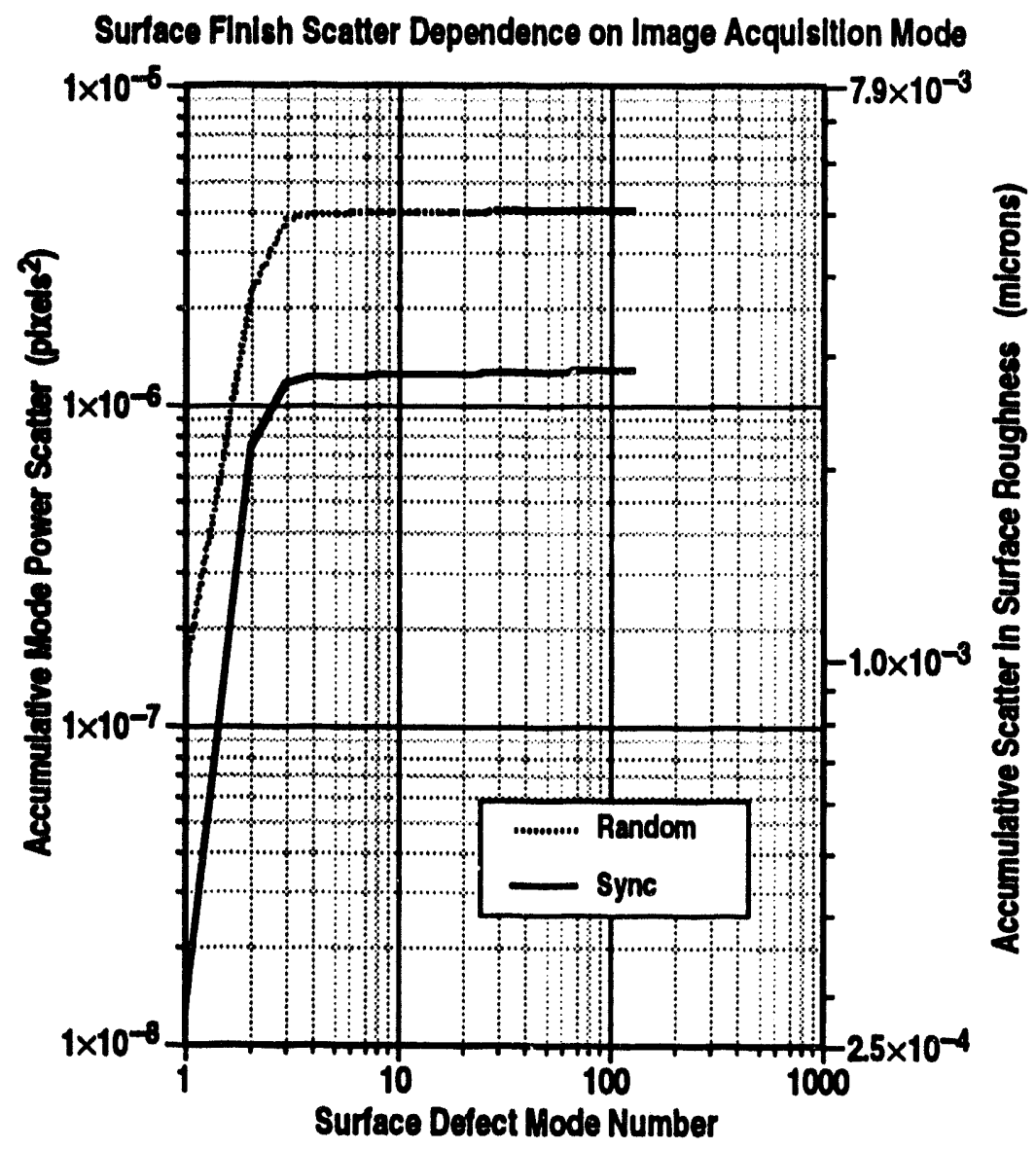

Fig. 3-3. Total cumulative (RMS) scatter in surface roughness for random and synchronized Image acquisition. Scatter is over ton images, and graph shows modal contribution to the scatter in surface roughness. Data is taken from empty cell images.

\subsubsection{DT SOLID LAYER EXPERIMENTS}

\subsubsection{1. $125 \mu \mathrm{m}$ DT SOLID LAYER}

The first DT layering experiment that we performed gave us a solid layer thickness of about $125 \mu \mathrm{m}$. For this experiment we were using our old incandescent $\mathrm{CW}$ illumination system, and were not synchronizing image acquisition to cryostat motion. We were, however, using the latest version of image analysis software with the nonlinear fitting algorithm. The 
goal of this experiment was to run long enough to allow the equilibration process to complete, then measure the DT layer surface finish.

The total running time for this experiment was nearly 46 hours. We set our cell temperature at $17.5 \mathrm{~K}$, although the actual cell temperature could have been a full degree warmer because of thermal effects of the $\mathrm{CW}$ incandescent illumination. During the first half of this experiment the illuminator remained on and we observed structural details in the solid layer that did not change over time. We then began turning the illuminator off between image acquisitions, and very quickly the layer structures began to disappear. We assumed our illumination system was supplying thermal energy to the cell which was somehow affecting the layering process. To minimize thermal perturbations of the solid layer, and minimize image smear due to cryostat motion during image exposure, we decided to replace our $\mathrm{CW}$ illuminator with a pulsed flash system.

The layer quality data for this experiment were reduced from the latest CCD images. Figure 3-4 shows the $125 \mu \mathrm{m}$ DT solid layer inside the copper cell, and Fig. 3-5 shows the

Fig. 3-4. CCD image of $125 \mu \mathrm{m}$ DT solid layer inside a $2 \mathrm{~mm}$ dlameter copper cell.

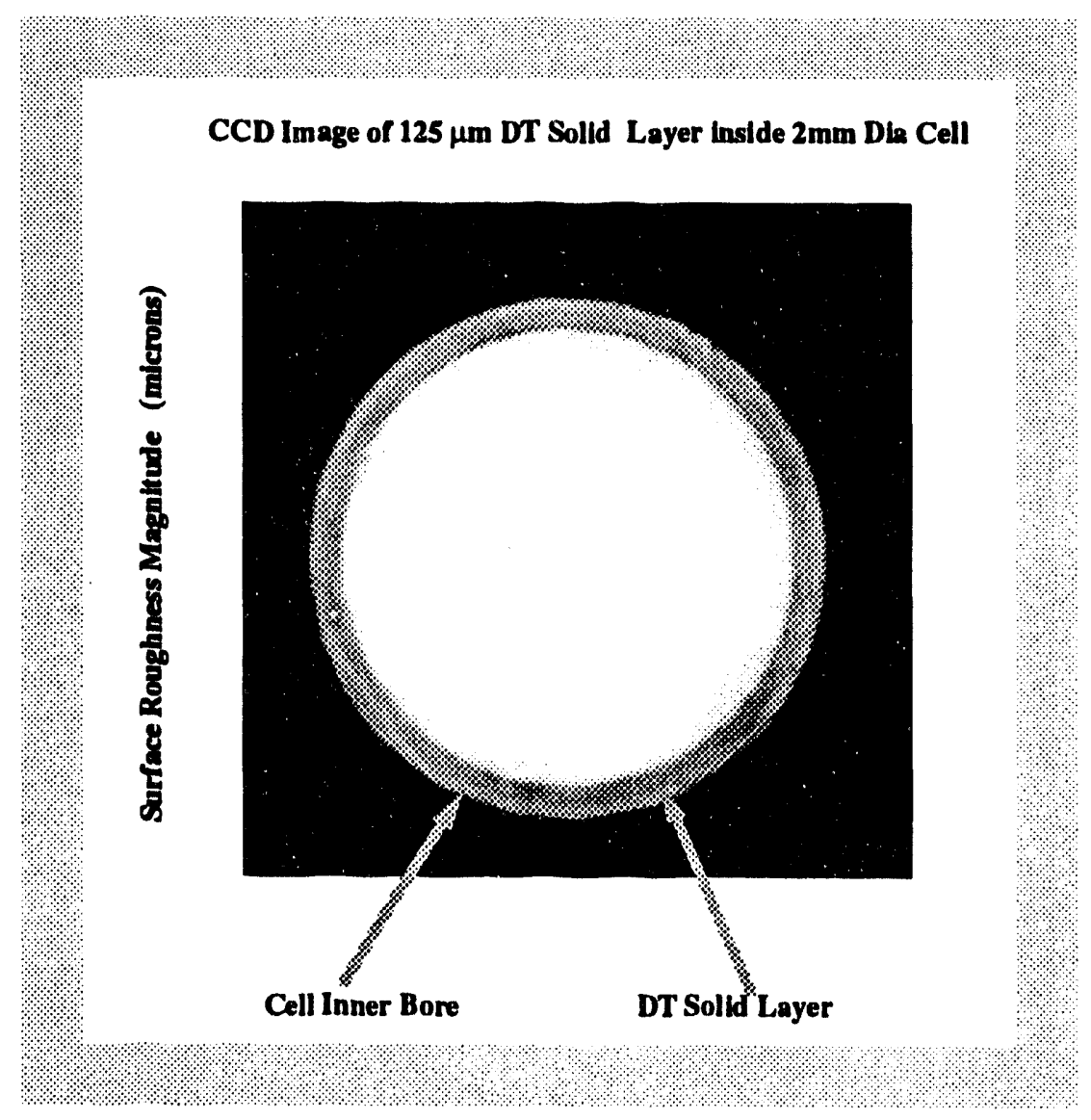




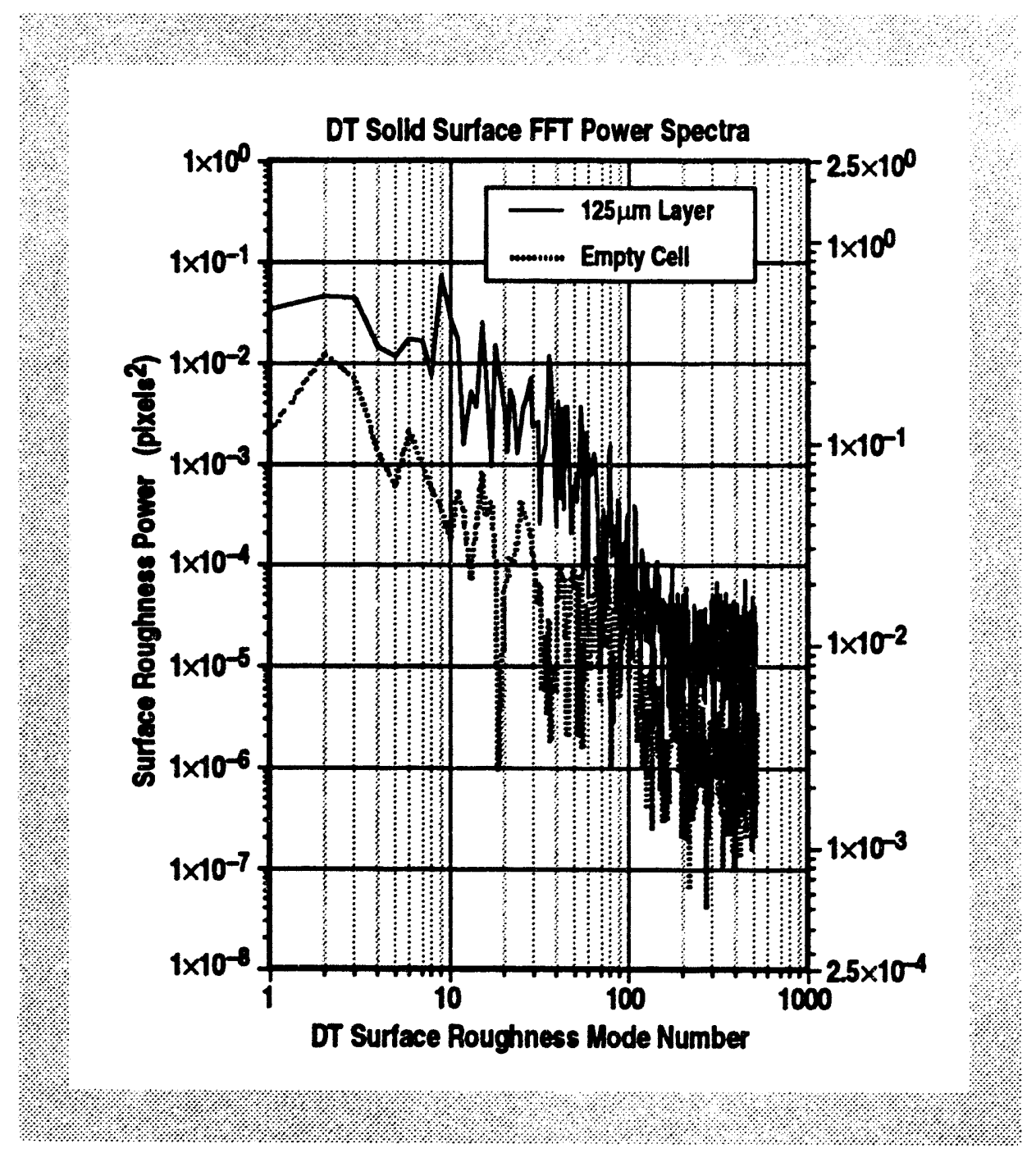

Fig. 3-5. FFT power spectra for $125 \mu \mathrm{m}$ DT solid layer and empty copper cell.

Fast Fourier Transform (FFT) power spectra for this DT surface and the empty cell surface. This graph shows the layer to be of very good quality, with all modal surface defects being less than $1 \mu \mathrm{m}$. The graphs in Fig. 3-6 show a surface roughness comparison between the $73 \mu \mathrm{m}$ and $125 \mu \mathrm{m}$ DT layers, and the time evolution of the $125 \mu \mathrm{m}$ surface finish [Refs. 3-5, 3-8]. Comparison of the data for the best layers obtained from both these experiments shows the $73 \mu \mathrm{m}$ layer had a surface roughness of just over $3 \mu \mathrm{m}$ rms at 19 hours, whereas the $125 \mu \mathrm{m}$ layer had a surface roughness of $1.8 \mu \mathrm{m}$ rms after 46 hours. This figure also shows the evolution of DT surface roughness for the $125 \mu \mathrm{m}$ layer, which is still improving after 46 hours. 


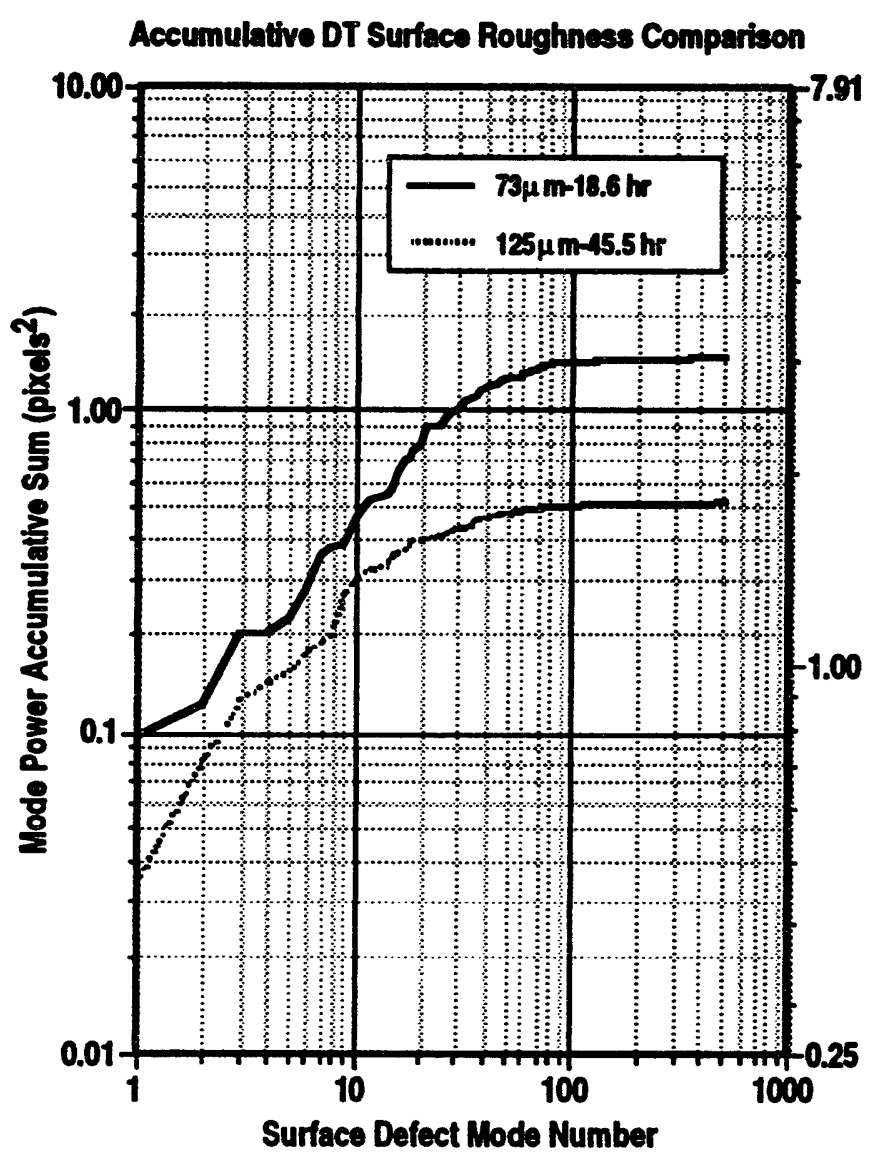

(a)

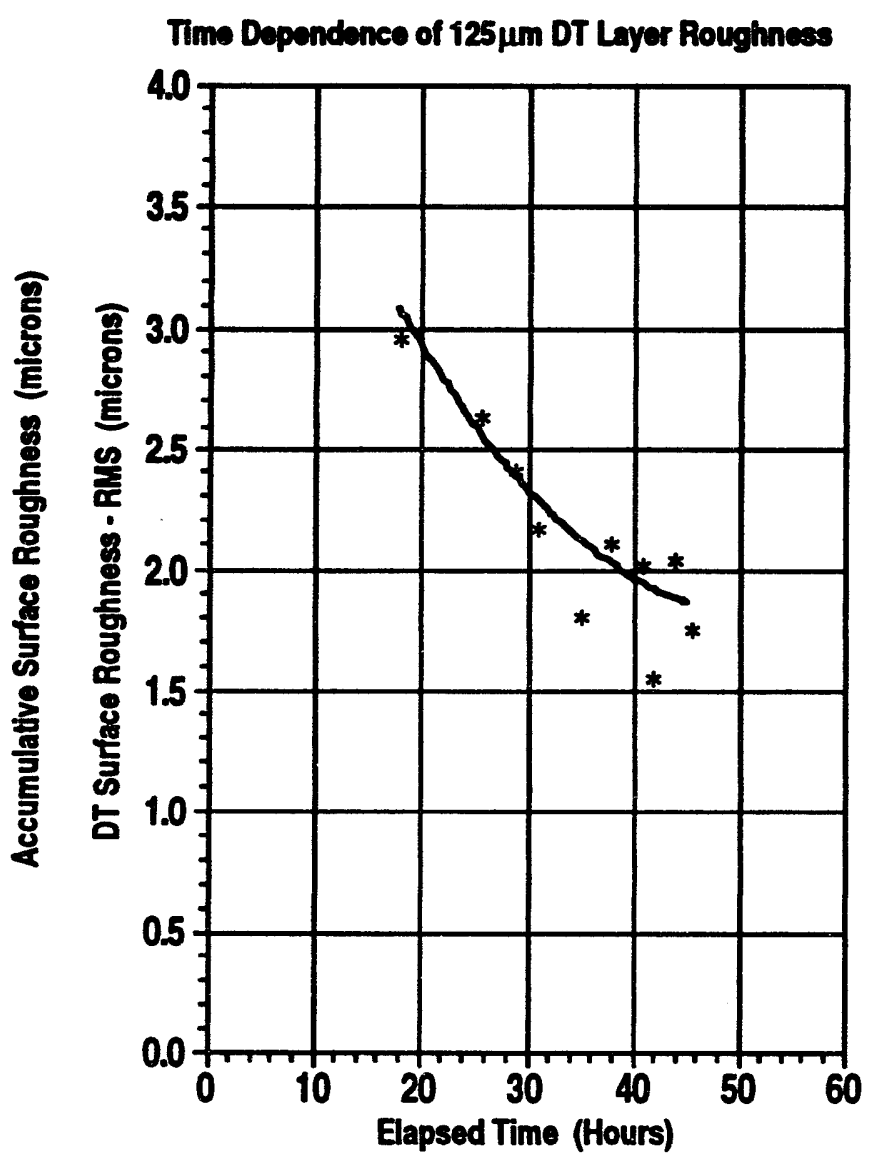

(b) 


\subsubsection{2. $\quad 139 \mu \mathrm{m}$ DT SOLID LAYER}

The next DT layering experiment we performed gave us a solid layer $139 \mu \mathrm{m}$ thick. This unusually thick layer was the result of replacing the incandescent illuminator with the pulsed flash illuminator system, and not operating CW at all. Normally we observed the DT fill in real time with a CCD camera mounted orthogonal to the main CCD array and the illuminator on $\mathrm{CW}$. The illuminator light thus warmed the cell slightly, producing a back pressure for the fill to work against. Consequently, a higher fill pressure was required to produce a given layer thickness. This time, without the $\mathrm{CW}$ illumination, less back pressure was produced and a $139 \mu \mathrm{m}$ layer resulted rather than the expected $125 \mu \mathrm{m}$ layer. During this experiment, we synchronized image acquisition to cryostat motion, using the new accelerometer and synchronized electronics to help improve solid layer image quality.

We also attempted to improve layer quality using an annealing process, whereby the solid layer temperature is increased in small increments and layer quality is measured as the temperature approaches the DT triple point. This process has been demonstrated in experiments performed using solid hydrogen films [Ref. 3-6]. Our results also looked promising, but we observed a low melting point for the DT layer that forced us to reexamine our experiment for clues about what may be happening.

We began this experiment at a layer temperature of $17.35 \mathrm{~K}$ and ran for 160 hours. We then increased the temperature to $18.32 \mathrm{~K}$ and ran for 23 hours, followed by another increase to $18.82 \mathrm{~K}$. Figures 3-7 through 3-10 show the results of this process. In Fig. 3-7 we show the image of the $139 \mu \mathrm{m}$ DT solid layer inside the copper cell at its best surface finish.Figure 3-8 is the FFT power spectrum of this DT surface, and the empty cell power spectrum. The best surface finish was produced at $18.82 \mathrm{~K}$, but after about 1.5 hours at this temperature, the DT surface began to degrade unexpectedly, never again to improve. After 56 hours at $18.81 \mathrm{~K}$, we increased the solid layer temperature to $19.01 \mathrm{~K}$, where the layer promptly melted.

Figure 3-10 shows the results of the entire process, including the time evolution of surface roughness at each of the selected layer temperatures, and the final optimum surface finish at each temperature. These data show the solid layer improving steadily with each temperature increase, until 1.6 hours after the temperature was set at $18.81 \mathrm{~K}$. Thereafter, the layer degraded (softened) steadily, and promptly melted once the temperature was set at $19.01 \mathrm{~K}$. 
Fig. 3-7. CCD Image of $139 \mu \mathrm{m}$ DT solid layer inside a $2 \mathrm{~mm}$ diameter coppor cell.

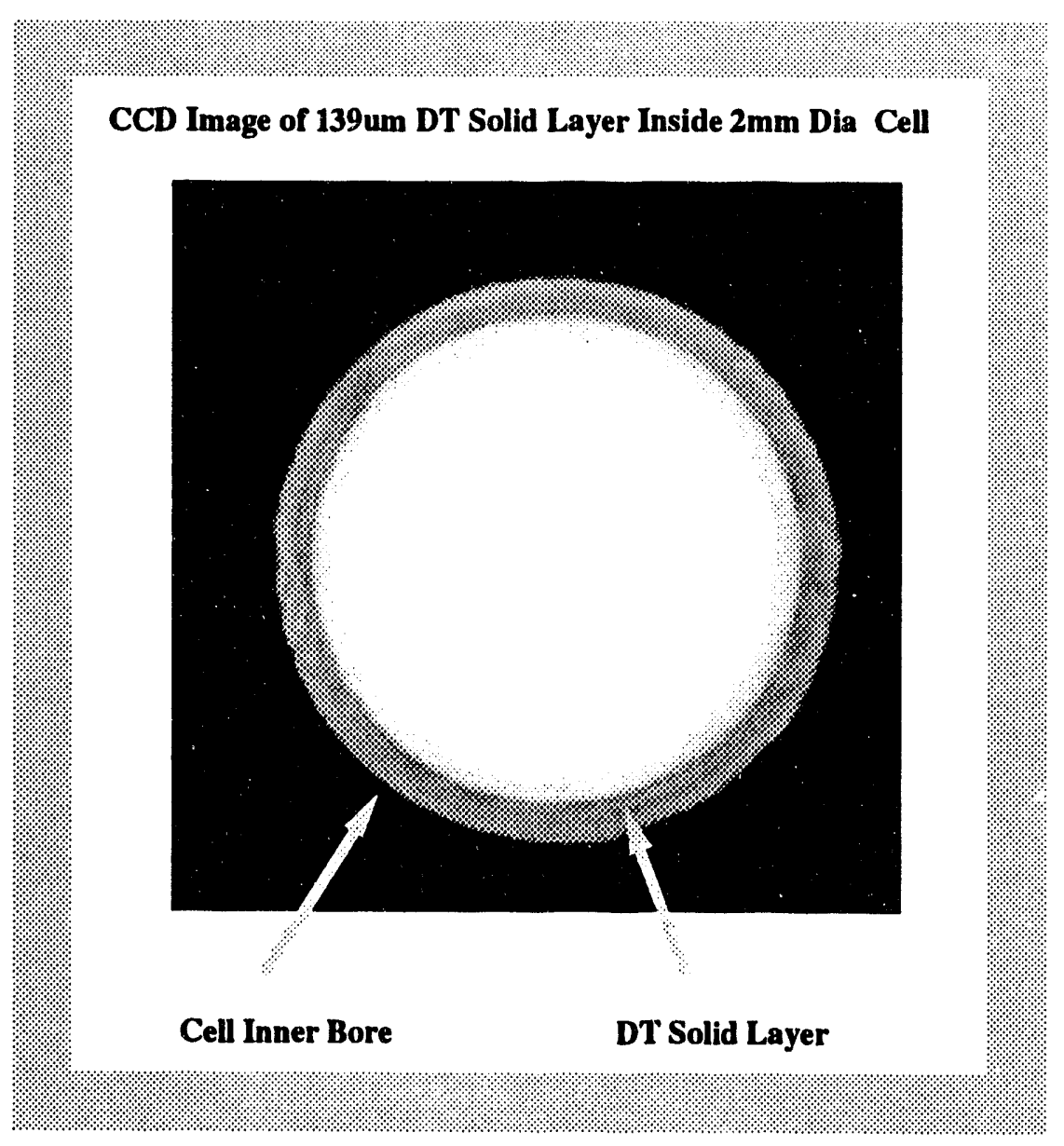

This DT solid layer appeared to be softening at $18.81 \mathrm{~K}$, which is coincidentally the melting point of pure $D_{2}$. With this in mind, we performed another experiment using pure $D_{2}$, and measured the melting point at $18.79 \mathrm{~K}$, showing that our thermometry is precise. The data from this $D_{2}$ experiment along with that from the $139 \mu \mathrm{m}$ DT layer experiment implies that we may be running a $D_{2}$ rich fuel mixture. If so, our equilibration time constant, and the DT layer surface finish, should improve once we run the correct 50-50 DT fuel mix.

The possible $\mathrm{D}_{2}$ rich mix could be the result of how we do our DT fills. Once we have modified our fill technique to produce a balanced DT mix, we should see further improvements in DT solid layer surface quality. For our next experiment, we will attempt to run a tritium rich fuel mix, and look for a decrease in the equilibration time constant and an improvement in layer surface finish. This will be followed by another experiment with the proper 50-50 DT fuel mix. If our suspicion of a $D_{2}$ rich mix is correct, we will see more improvement in DT solid layer quality. 


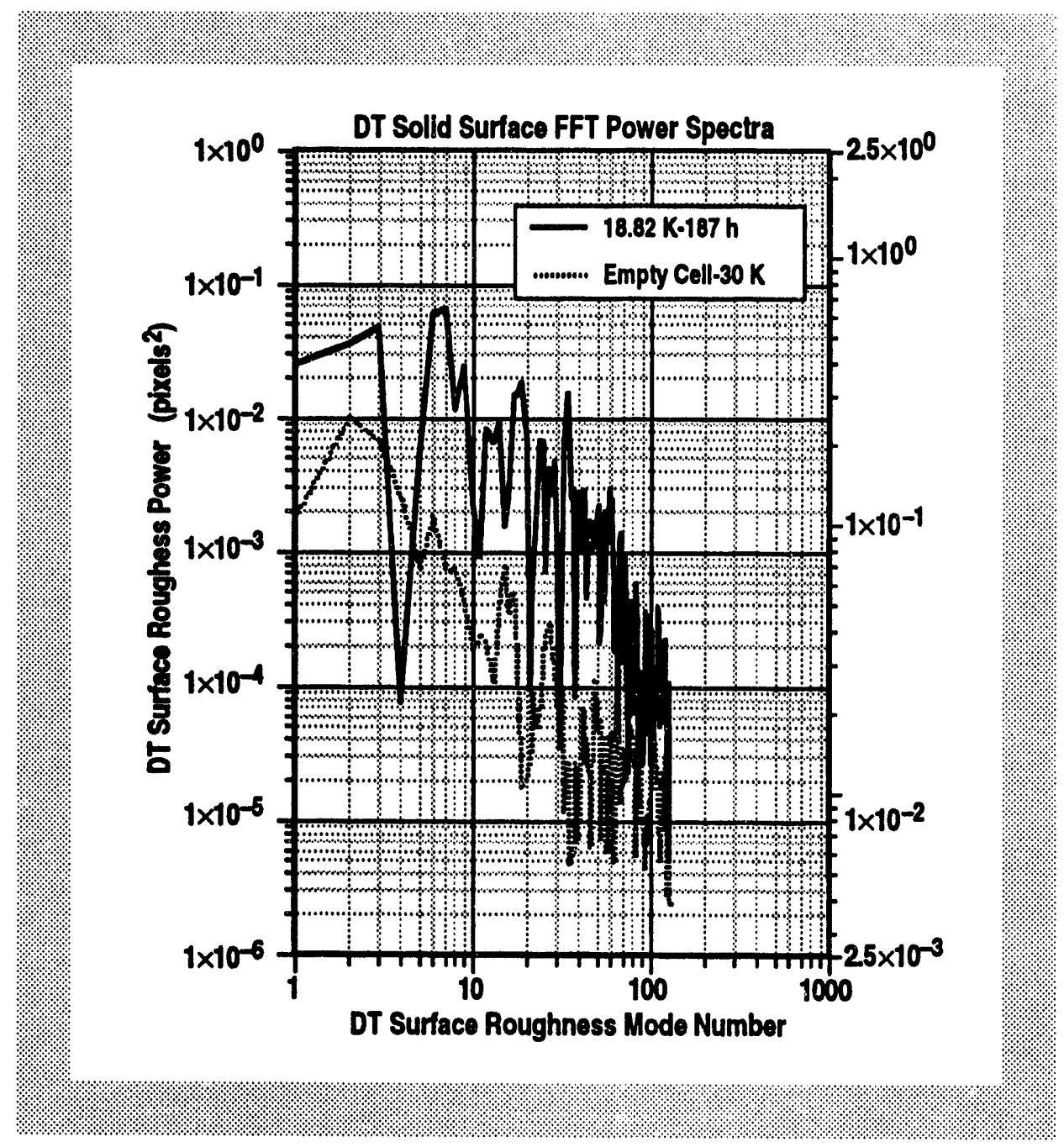

Fig. 3-8. Solid surface power spectra for $139 \mu \mathrm{m}$ DT solid layer and empty copper cell. DT layer spectrum is for best layer obtained at 18.8K.

\subsubsection{CONCLUSIONS}

The results of these experiments show that DT solid layers can achieve surface finishes (roughness) of at least $1.7 \mu \mathrm{m}$ RMS, and that it is possible to improve the surface finish through the process of annealing. Although these DT surface finishes are still a factor of three rougher than that required by the proposed NIF target [Ref. 3-6], we have reason to believe that we can improve layer surface quality even further.

For further information, please contact J. Simpson (GA). 


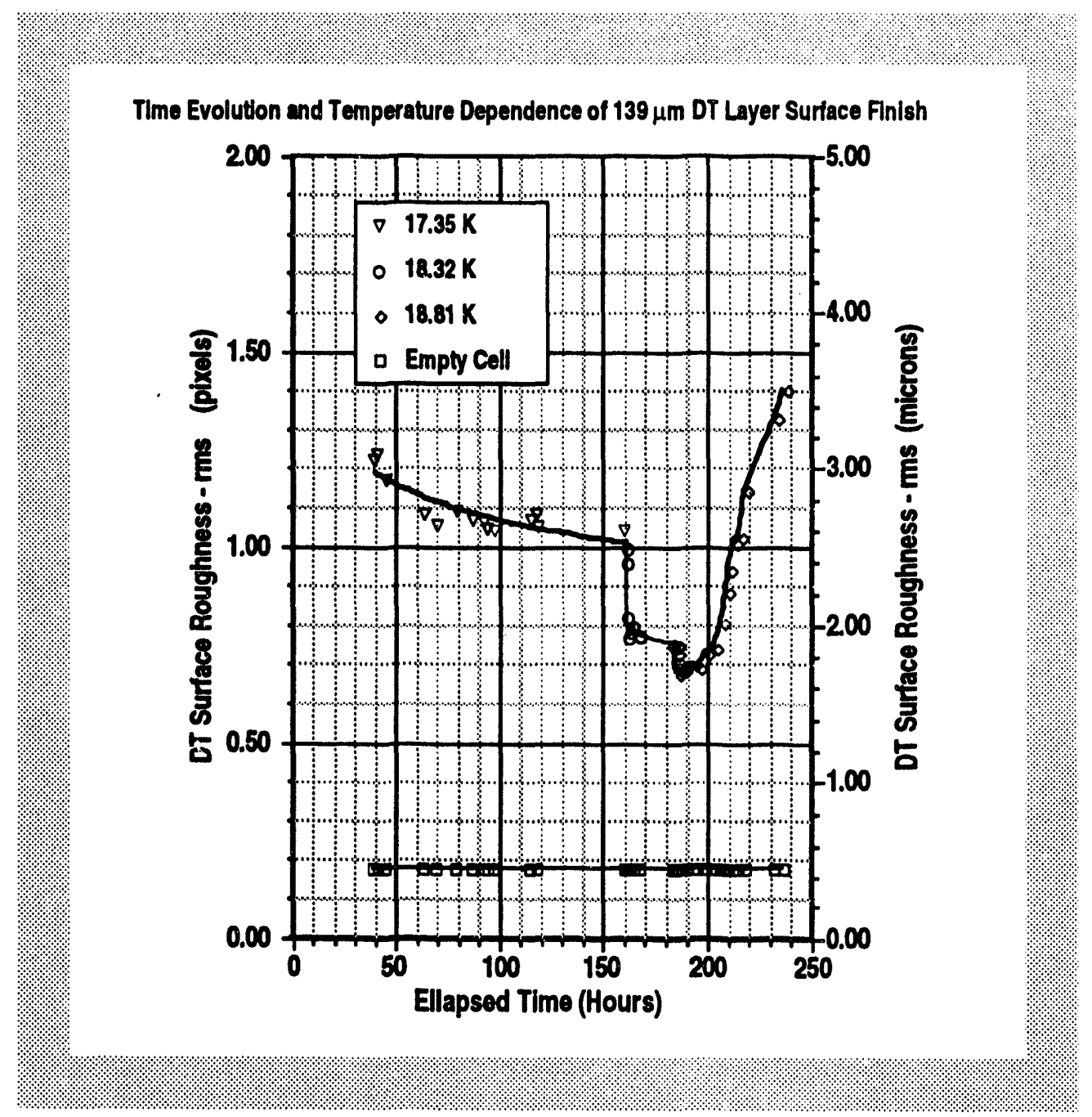

Fig. 3-9. Time evolution of $139 \mu \mathrm{m}$ DT solid layer surface finish, at three temperatures. Also shown is the constant surface finish of the empty copper cell.

\subsection{CHARACTERIZATION DEVELOPMENT AT LANL}

Characterization development at LANL is centered on two projects: Resonant Ultrasound Spectroscopy (RUS) and 1-Dimensional spatially-resolved Nuclear Magnetic Resonance spectroscopy (1-D NMR). John Simpson and Jane Gibson of GA performed onsite activities in support of the RUS research. Neil Alexander of GA performed onsite activities in support of the 1-D NMR experiments. 


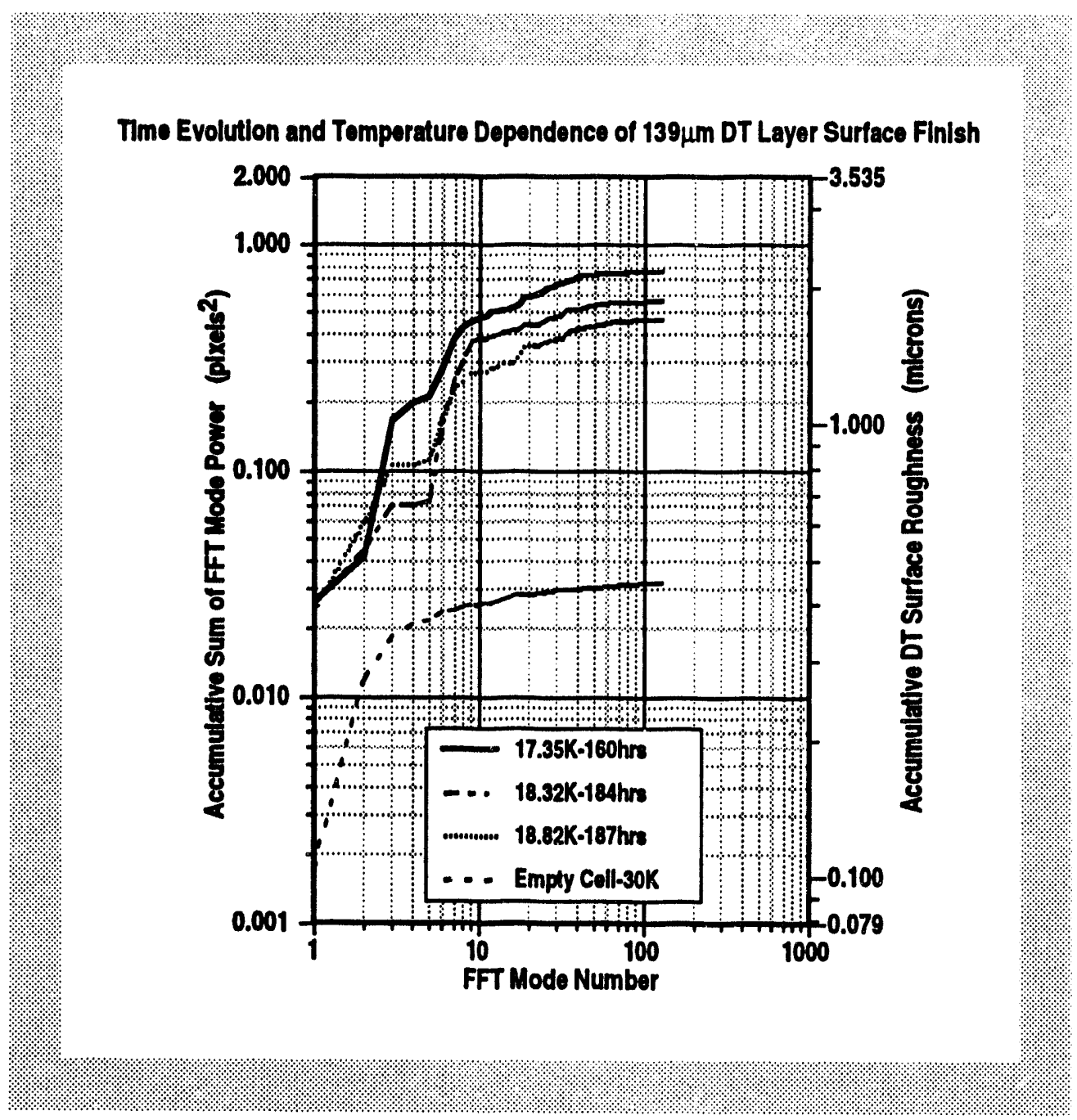

Fig. 3-10. Cumulative (RMS) surface finish of the $139 \mu \mathrm{m}$ DT solid layer at three temperatures. Also shown is cumulative (RMS) surface finish of the empty copper cell.

\subsubsection{RESONANT ULTRASOUND SPECTROSCOPY}

Our task was to assist LANL in measuring $\mathrm{D}_{2}$ and DT phase transitions inside opaque cryogenic targets using RUS.

Initial experiments were designed to test RUS for its ability to sense gross changes in target geometry. Can it distinguish a solid sphere from a sphere with a hole drilled through it? Can it observe a distinction between a spherical aluminum shell filled with high pressure $D_{2}$ gas and the same shell cooled sufficiently to condense the $D_{2}$ ? 
The RUS uses a set of transducers contacting the target sphere to induce and detect resonant ultrasonic vibrational modes of the target. One transducer is used to force the target through a range of ultrasonic frequencies. The other transducers detect the amplitudes of vibration at these frequencies at specific locations on the target. The highly symmetric nature of the spherical target makes it very difficult to predict or establish the location of the target's vibrational nodes with respect to the small number of receiving transducer locations. The magnitude of vibration induced in a receiving transducer will vary depending on how close to a node that receiver is. Therefore, the frequencies at which resonances occur are the only ones that can be unambiguously determined.

We improved the RUS in a number of ways this year. We replaced the old pair of transducers with a set of four miniature transducers, denoted as pinducers. They are arranged in a tetrahedral configuration with the top pinducer used to drive the target and the bottom three used as receivers, as shown in Fig. 3-11.

We mounted the pinducers in a cryostat, cooled with a CR Cryogenics (Model 22) closed-cycle refrigerator. To reduce noise induced from the electrical and mechanical systems of the refrigerator, we electrically isolated the receiver electronics and added a low frequency noise reduction circuit. We completed the data acquisition hardware and software and wrote spectrum analysis software.

Fig. 3-11. The RUS pinducers are arranged around the targot in a totrahodral configuration.

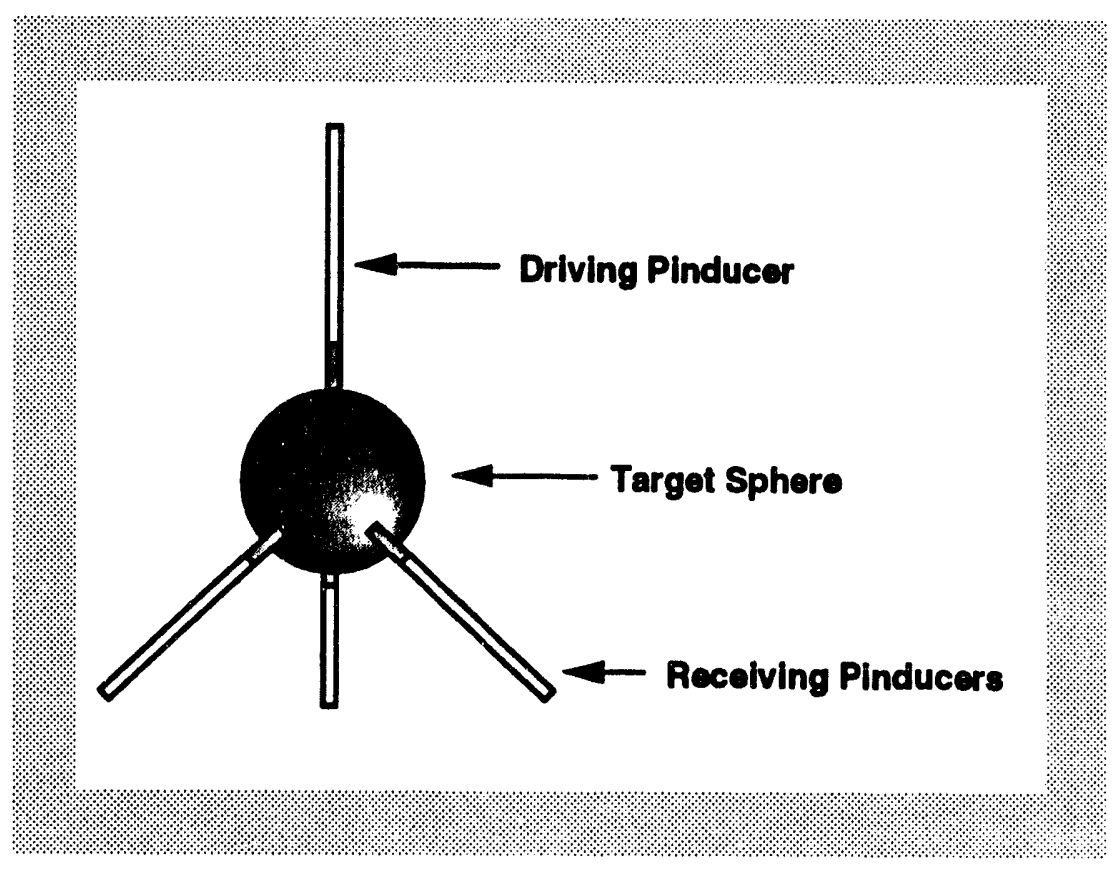




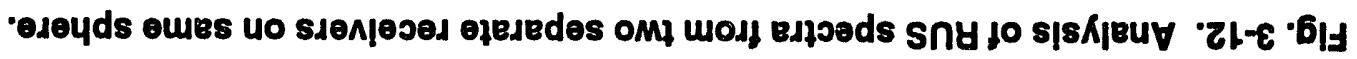

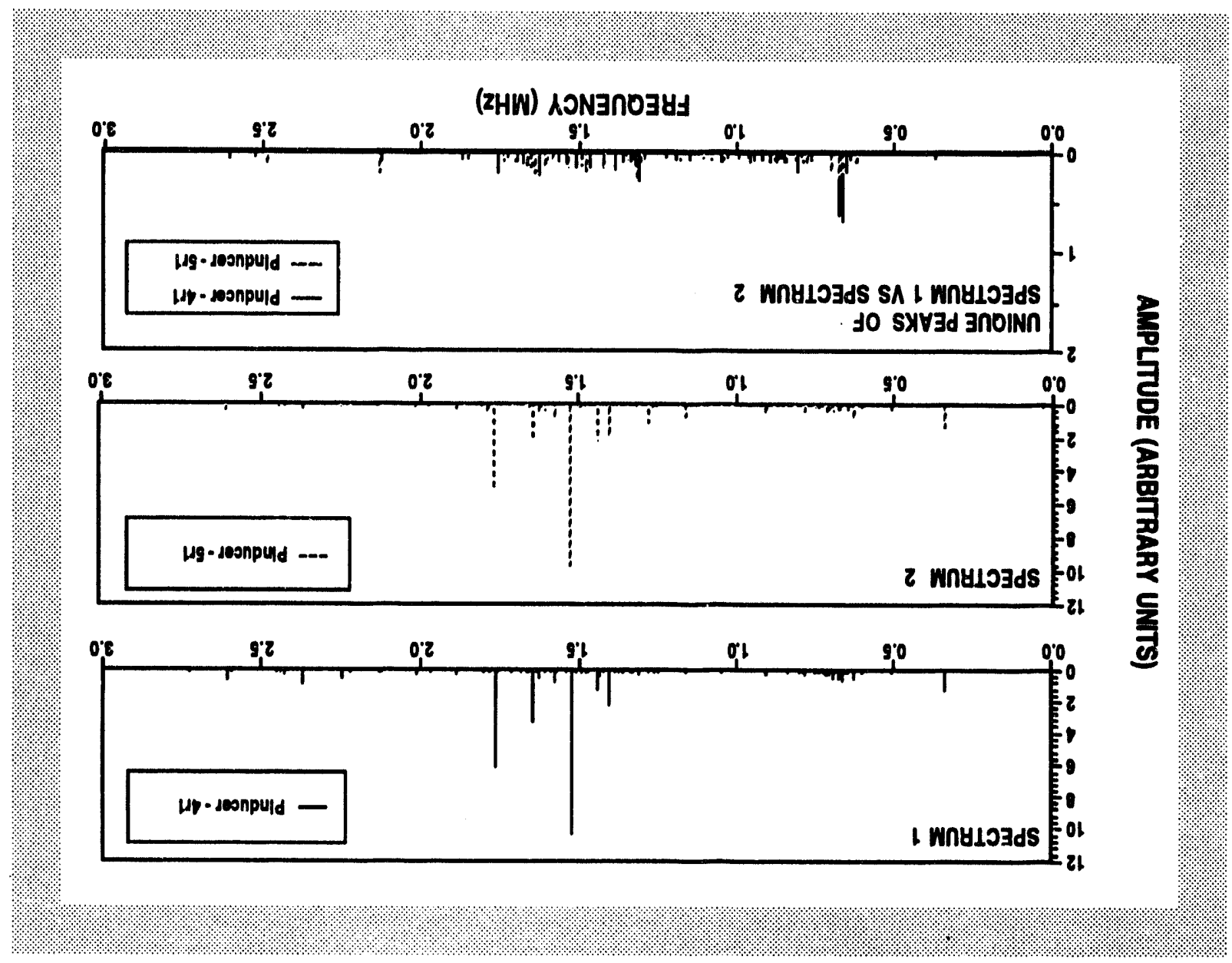

-(วoвjuns วul uo

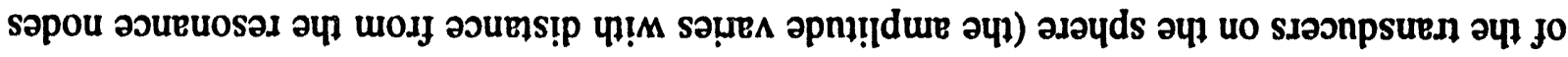

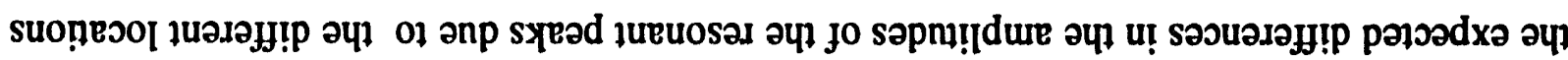

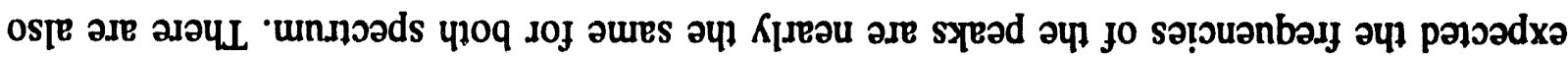

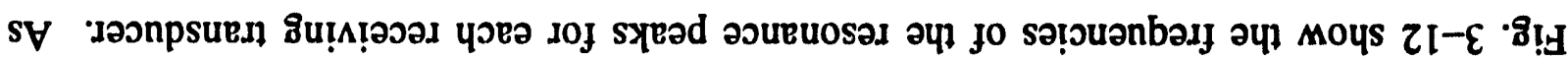

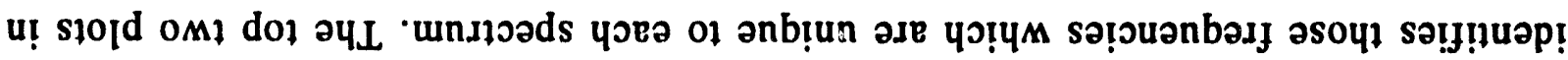

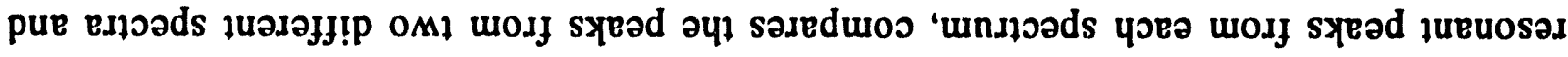

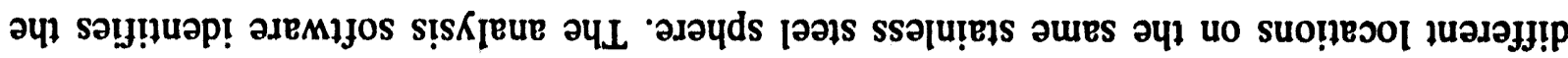

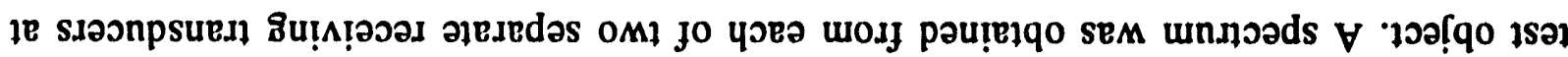

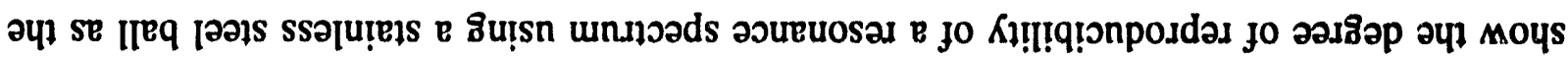

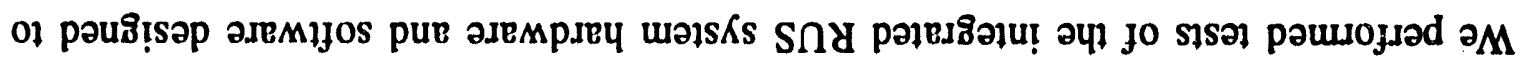


In later target characterization studies it will be very useful to be able to compare target spectra with "reference" spectra. For the bottom plot in Fig. 3-12, the analysis software compared the top two spectra and identified the peaks that are unique to each (note the difference in the vertical scale). All frequencies which were unique to either spectrum have an amplitude of less than "one". All resonances with amplitude greater than "one" are seen by both pinducers.

The RUS system is almost ready to be put to use in looking for the phase transition that occurs upon cooling a deuterium-filled aluminum sphere. The major modification left to be implemented is attaching sapphire micro-tips on the pinducers. This will improve the coupling of the pinducers with the target. With the completion of this modification, the RUS system will be ready for cryogenic tests.

For further information, please contact J. Simpson or J. Gibson (GA).

\subsubsection{SPATIALLY-RESOLVED NUCLEAR MAGNETIC RESONANCE}

Our task was to assist LANL to implement NMR experiments on DT-filled, beta-layered targets.

Our initial NMR experiment was designed to test if the beta-layering process [Ref. 3-9] produces a DT layer that has $D$ and $T$ uniformly distributed throughout the layer. If the ratio of $D$ to $T$ varies, the density in the target's fuel layer will be non-uniform. This asymmetry would impede target performance during an implosion.

There are several pathways to produce fractionation in DT during beta-layering. The manner in which the initial puddle of slumped DT is frozen in the target can produce an initial fractionation in the starting solid before beta-layering begins [Ref. 3-10]. The thermal velocities of the DT species $\left(D_{2}, D T, T_{2}\right)$ and the partial pressures of the species in the vapor above the solid are substantially different. Deuterium tends to be preferentially deposited at the beginning of the beta-layering process.

We will use 1D-NMR to determine the number of tritons and deuterons at each elevation within a beta-layered DT sample. If the ratio of $D$ to $T$ varies with elevation, then fractionation has occurred and beta-layering will have produced a fuel layer with asymmetric density. Such a result is illustrated in Fig. 3-13 along with the conceptual design of 1-D NMR 
D-T sample: $8 \mathrm{~mm}$ tall $\times 5 \mathrm{~mm} \varnothing \times 500 \mu \mathrm{m}$ thick, $113 \mathrm{Cl}$ in cell, 150 - $250 \mathrm{Cl}$ including fill system.

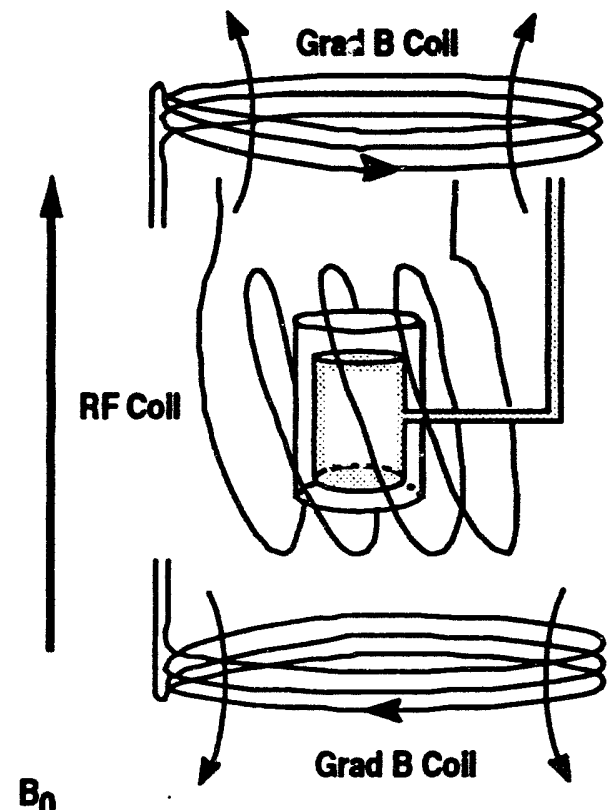

4.7 tesla

Field Gradient Encodes Spatial Information as

$$
\Delta \mathbf{z}=\frac{\Delta \mathbf{v}}{7 \mathrm{G}_{2}}
$$

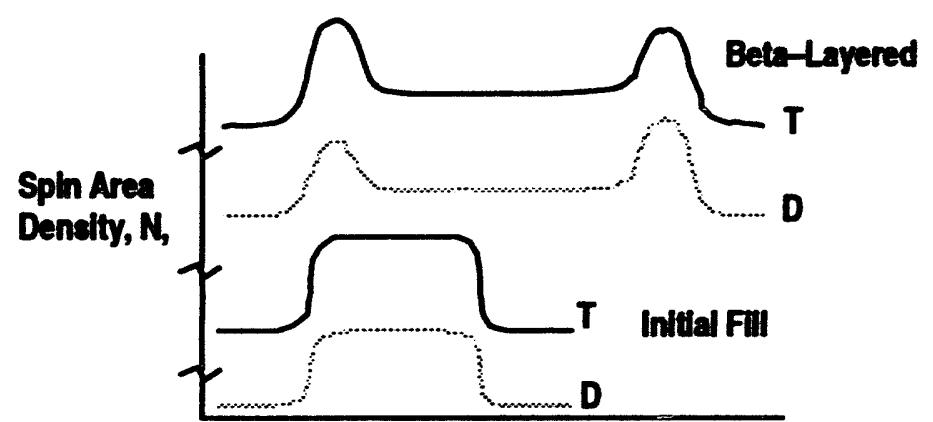

$G_{2}=d B / d z$

For Resolution

of $-0.5 \mathrm{~mm}$

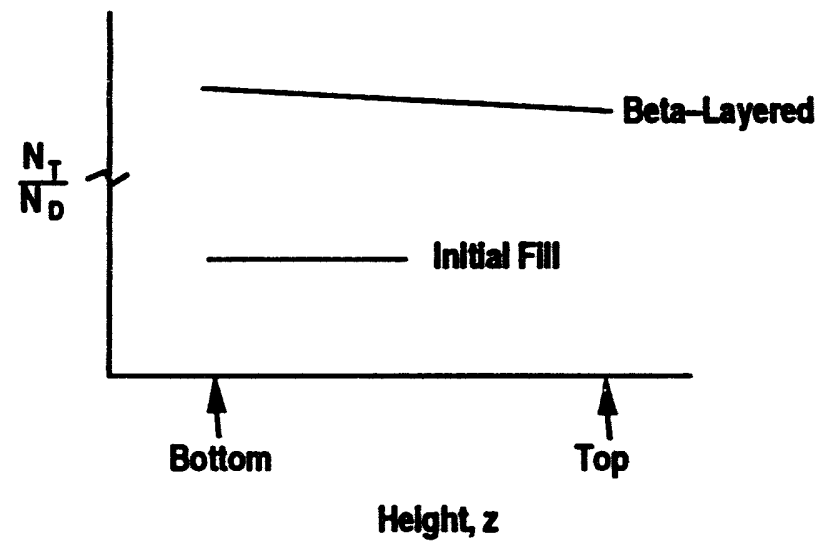

Relative Frequency, $n$

- Will beta-layering give spatially uniform $T$ to $D$ density ratio?

Fig. 3-13. Experimental concepts for spatially resolved NMR of beta-layered DT. 
equipment. To produce a spatially resolved NMR signal in the vertical direction, we added a set of coils to the usual RF coil to make the magnetic field gradient vertical and uniform. With the field gradient added, each RF frequency corresponds to a separate elevation within the sample. Elevation is given by the frequency divided by the field gradient divided by the magneto-gyric ratio of the nuclei under observation. The magneto-gyric ratio of $D$ and $T$ are substantially different, which makes them easily distinguished with NMR. The spatial resolution is given by dividing the NMR line-width by the field gradient in appropriate units. In solid DT at a temperature near the triple point, a field gradient of about $9 \mathrm{G} / \mathrm{cm}$ will provide a resolution of $0.5 \mathrm{~mm}$. The sapphire cell that will be used in this experiment has an inside diameter of $5 \mathrm{~mm}$ and a height of $8 \mathrm{~mm}$. The field gradient will easily be able to distinguish the DT at the top and bottom of the cell.

We used a Varian Unity 400 NMR spectrometer [Ref. 3-11] with a 9.4 tesla warm-bore magnet to gain experience with imaging protons in liquids and solids, including molecular hydrogen, and to test field gradient coil designs. The disassembled major components are shown in Fig. 3-14. On the far left is the warm-bore superconducting magnet. To its

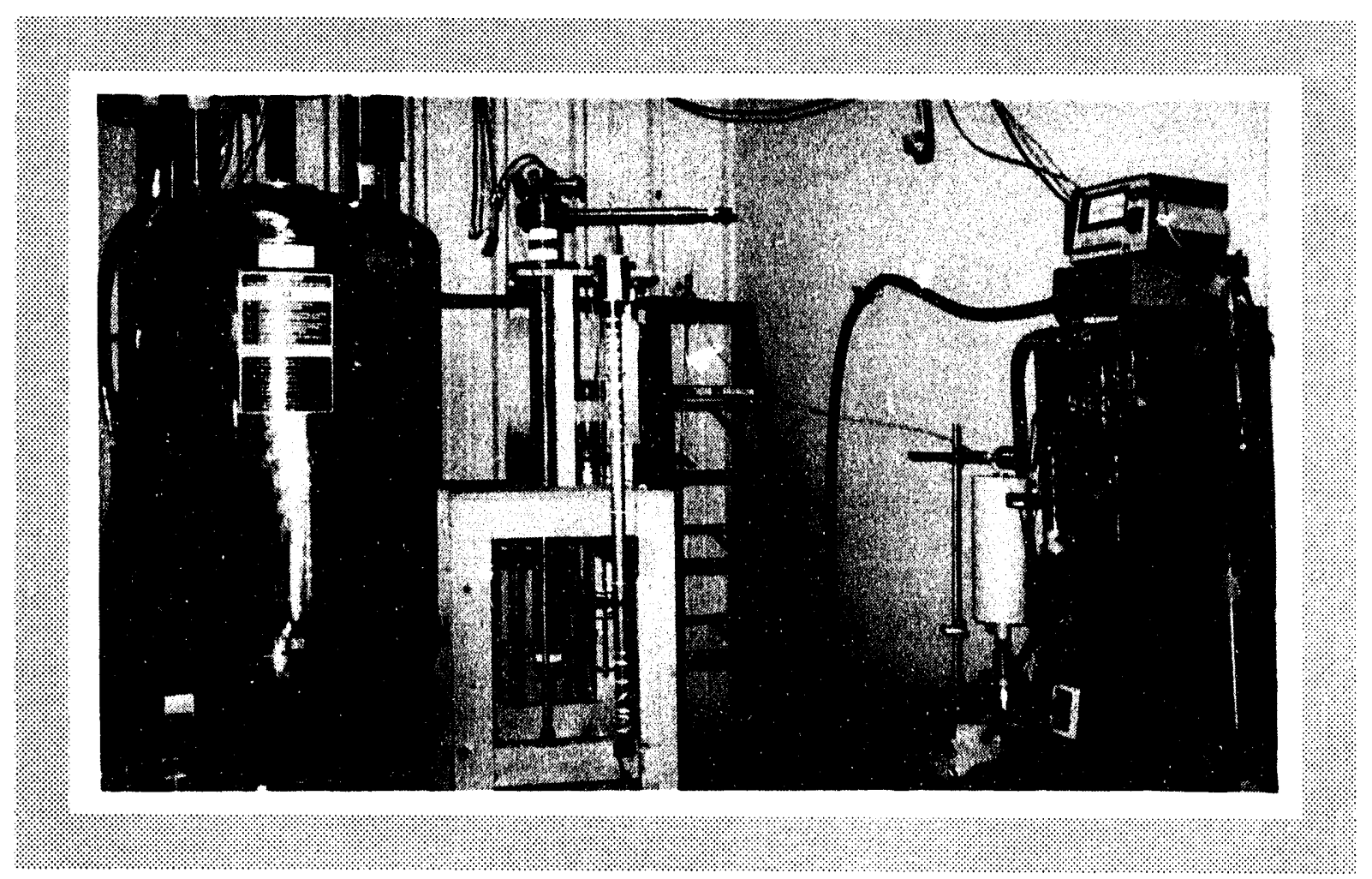

Fig. 3-14. NMR probe and cryostat are shown removed from the magnet. 
immediate right is the Oxford continuous flow liquid helium cryostat used to cool the NMR probe. The probe is to the right of the cryostat and is shown without a sample in the RF coil and with the gradient coil removed. The probe is normally inserted in the cryostat, which is inserted into the magnet. The probe body consists entirely of a 1.25 inch diameter, $75 \Omega$ impedance, plated stainless steel transmission line. The RF and gradient coils are located at the bottom of the transmission line. The transmission line is topped by an RF tuning and matching network. The sample cell is filled via a non-conducting tube fed through the inside of the inner conductor of the transmission line. Another spectrometer is available in a facility cleared for tritium work. It is a Bruker CXP $213 \mathrm{MHz}$ spectrometer with a 4.7 tesla warm-bore superconducting magnet. We purchased an identical cryostat for this magnet and built a prototype of a doubly tuned NMR probe for this spectrometer. It successfully resonated at $213 \mathrm{MHz}$ and $30.7 \mathrm{MHz}$ for use with $\mathrm{T}$ and $\mathrm{D}$ respectively. Interchannel isolation was $36 \mathrm{~dB}$. The prototype used a similar design to that described above, but we added another frequency channel by tapping the transmission line at a node near the top of the line. We attached the lower frequency matching network to the line's inner conductor at this node.

We used two pulse sequences to collect 1-D NMR images. One sequence was the field gradient Free Induction Decay (FID). The other was the field gradient Hahn echo with a four-cycle phase rotation to cancel asymmetries in the quadrature detection of the signal. The latter gives more accurate results and uses less RF power but requires faster decay of the field gradient coils. Using the probe without the cryostat, 1-D images were made using the field gradient Hahn echo. A typical result is shown in Fig. 3-15. This is a vertical 1-D projection of the protons in water contained in five horizontally oriented tubes. Each tube is $1.0 \mathrm{~mm}$ in diameter with its axis $2.8 \mathrm{~mm}$ above the adjacent tube's axis. When the probe was placed into the cryostat, the field gradient coil decay slowed, forcing us to use the field gradient FID to image low temperature specimens. A typical result is shown in Fig. 3-16. This is a vertical 1-D projection of the protons in a solidified puddle of normal hydrogen and in the gaseous hydrogen from the vapor pressure above the solid. The sample cell is a cylinder with its axis lying horizontal. The sharp peak at the bottom of the cell is the projection image of the solid. The broad low lying component is the projection of gas. Note that the broad component is at its maximum at the center of the cell, reflecting the fact that a cylinder lying on its side has a maximum cross-sectional area (and hence maximum number of hydrogen molecules) at its midpoint elevation. 


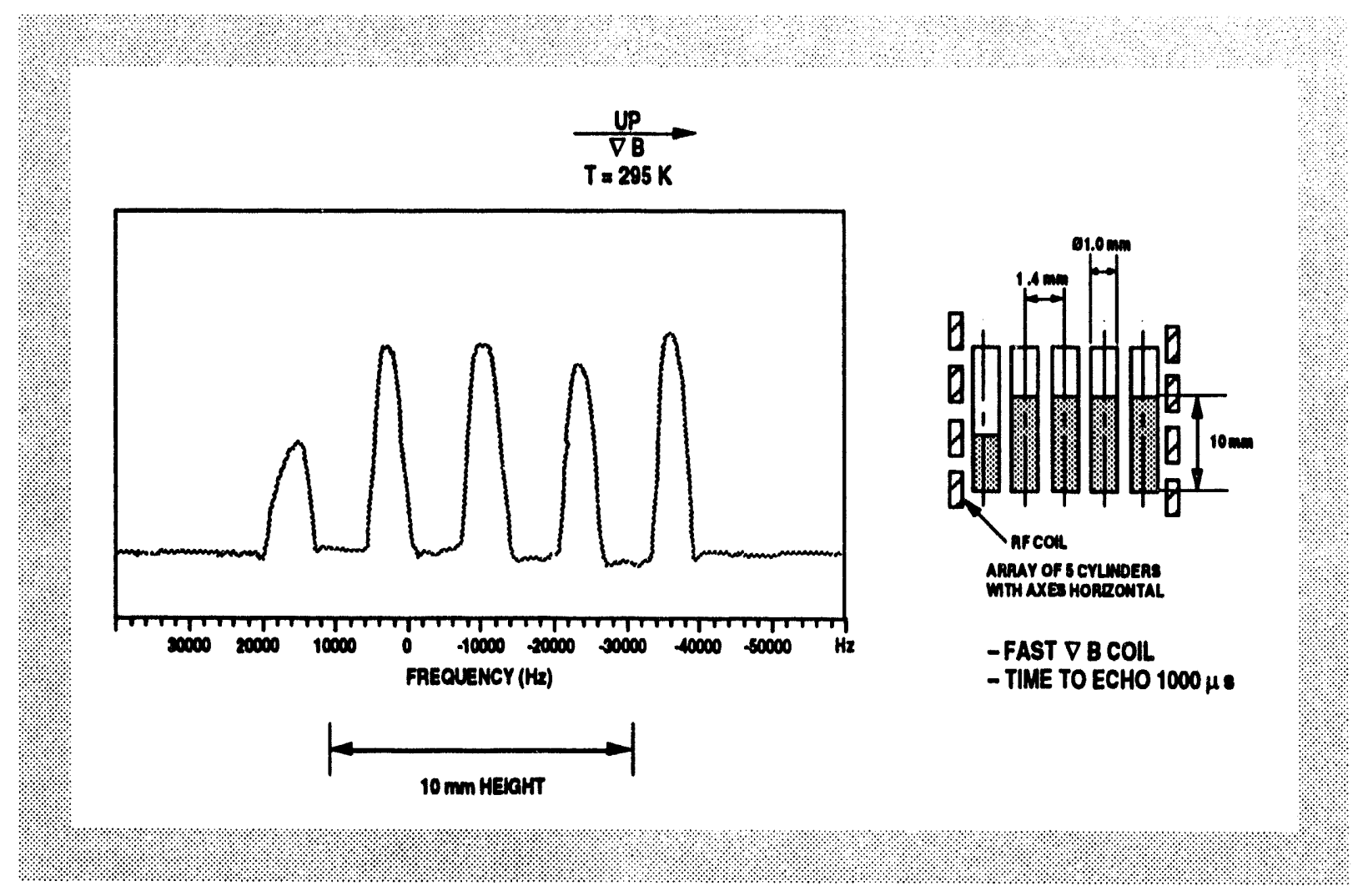

Fig. 3-15. Proton 1-D projection Image of water contained in five horizontally oriented tubes using the gradient Hahn echo.

We will have to increase the speed of the field gradient coil to enable the use of the more accurate field gradient Hahn echo in the cryostat. This can be done in two ways. Since the speed loss of the coil is probably due to the induction of eddy currents in the walls of the cryostat, the use of a coil of self-shielded design should improve speed. The coil speed can also be improved by adding a "pre-emphasis" circuit to the coil driver. This over-drives the coil until it produces the desired field and then levels off the driving potential.

For further information, please contact Dr. Neil Alexander (GA).

\subsection{CHARACTERIZATION DEVELOPMENT AT LLNL}

Jim Sater and Don Bittner of W. J. Schafer Associates together with Jon Larsen of Cascade Applied Sciences, Inc. supported characterization development at LLNL. 


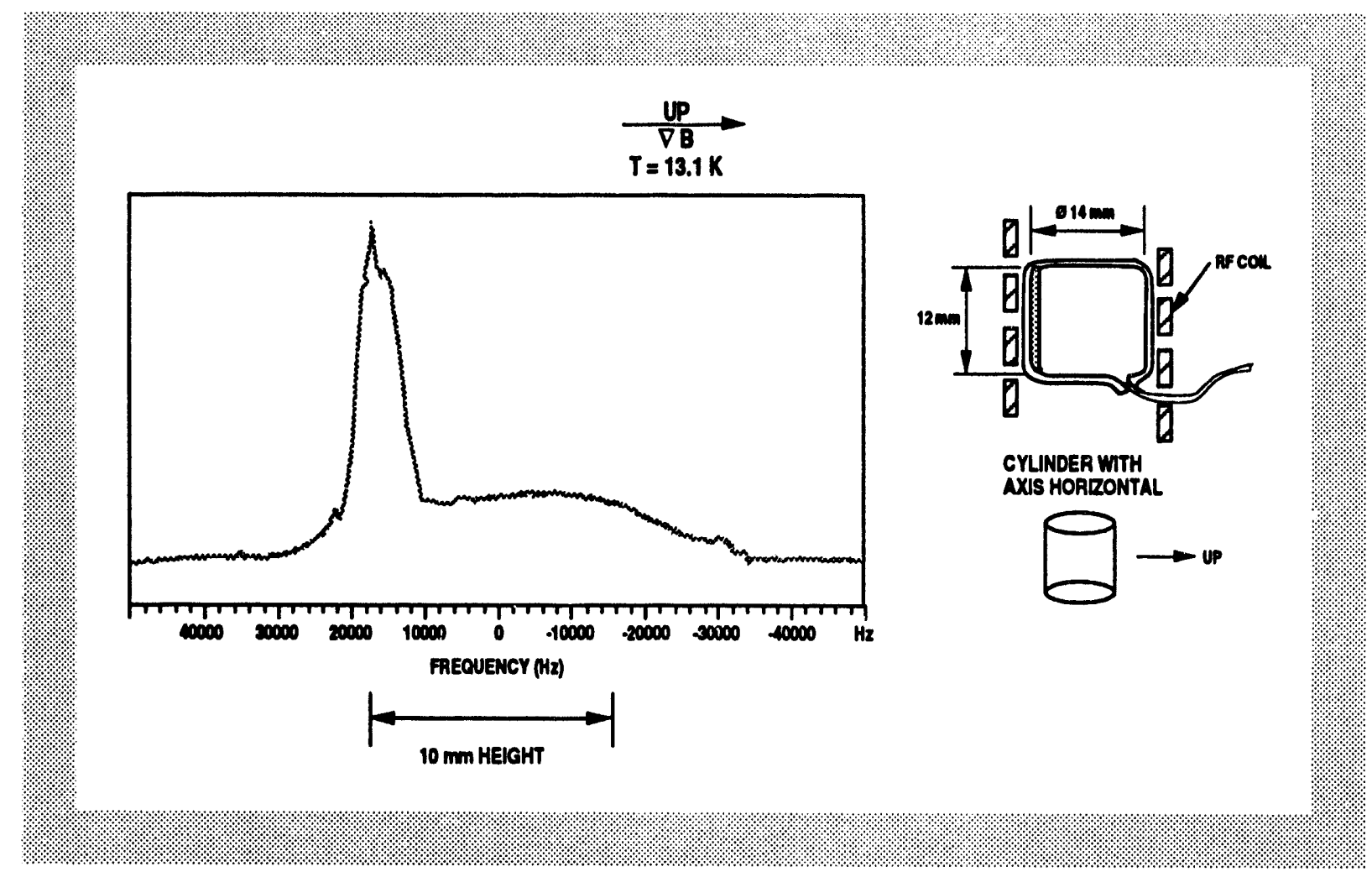

Fig. 3-16. Proton 1-D projection image of solidified- $\mathrm{H}_{2}$ and gaseous- $\mathrm{H}_{2}$ above the solid using the gradient Free Induction Decay (FID).

\subsubsection{CALORIMETRIC MEASUREMENT OF FUEL FILL}

Calorimetric measurement of fuel fill in ICF capsules is being pursued as an alternative to current optical or destructive measurement techniques [Ref. 3-12]. The advantages of measuring fill by observing a thermal signature are that the measurement becomes independent of the operator's visual acuity and, more importantly, it offers the means to make measurements on opaque capsules.

The calorimetric fuel measurement technique is an adaptation of the principle used in LLNL's dew point machine [Ref. 3-13]. As hydrogen gas inside a capsule is cooled it condenses at a temperature between the triple point and the critical point of the gas (18.7 K and $38.3 \mathrm{~K}$ respectively for $\mathrm{D}_{2}$ ). The temperature at which the gas condenses to liquid is dependent on the isotopic composition of the gas and on the fill pressure. If we can measure this "dew point" temperature and we know the isotopic mixture of the hydrogen fuel, we can determine the fill pressure. 
The heat capacity of the gas being cooled through the dew point undergoes a large discontinuous change. The heat capacity of the gas at temperatures above the dew point is much smaller than that of the gas/liquid mixture below the dew point. We use a calorimetric technique to look for this change in heat capacity.

The method we chose to measure capsule heat capacity is known as "ac" calorimetry [Ref. 3-14]. We apply a sinusoidal heat input to the sample. We then measure, using phase sensitive detection, the ac component of the sample temperature $\left(\mathrm{T}_{\mathrm{ac}}\right)$. The magnitude of $T_{a c}$ is inversely proportional to the heat capacity of the sample. The advantage of this technique is that it can produce a very good signal-to-noise ratio and therefore is sensitive to small changes in heat capacity. We can also relax requirements for thermal isolation between the calorimeter and the surroundings as compared to more classical techniques like adiabatic calorimetry [Ref. 3-15].

We measured the fuel fill of an $800 \mu \mathrm{m}$ glass shell. This shell was listed as having been originally filled with 51.1 atmospheres of $D_{2}$ gas. Before beginning our experiment, we measured the dew point using the LLNL dew point machine. The transition temperature was found to be $29.21 \mathrm{~K}$. This corresponds to a room temperature fill pressure of 49.3 atmospheres. The results of the calorimetric measurement are shown in Fig. 3-17. The transition is readily visible. A fit by eye on an expanded view of the data gave a transition temperature of $29.02 \mathrm{~K}$. We calculated the fill pressure to be 47.4 atmospheres. This agrees with the dew point measurement to within $4 \%$.

We are attempting to get better agreement between theory and data. The qualitative shape of the data curve is exactly what we expected but the absolute magnitudes of the heat capacity data appear to be a factor of ten too low. We believe that this is due to our assumption that the sample has an infinitely small thermal relaxation time. Another area that warrants further investigation is the behavior of the sample as we approach the critical point. We have taken data on a capsule filled with $\mathrm{H}_{2}$ near the critical pressure but were unable to measure its dew point. It may be important to know how close to the critical point the calorimetric technique can be used. Finally, work needs to be done to build an apparatus capable of doing calorimetry on a production basis.

For further information, please contact Dr. J. Sater (WJSA). 


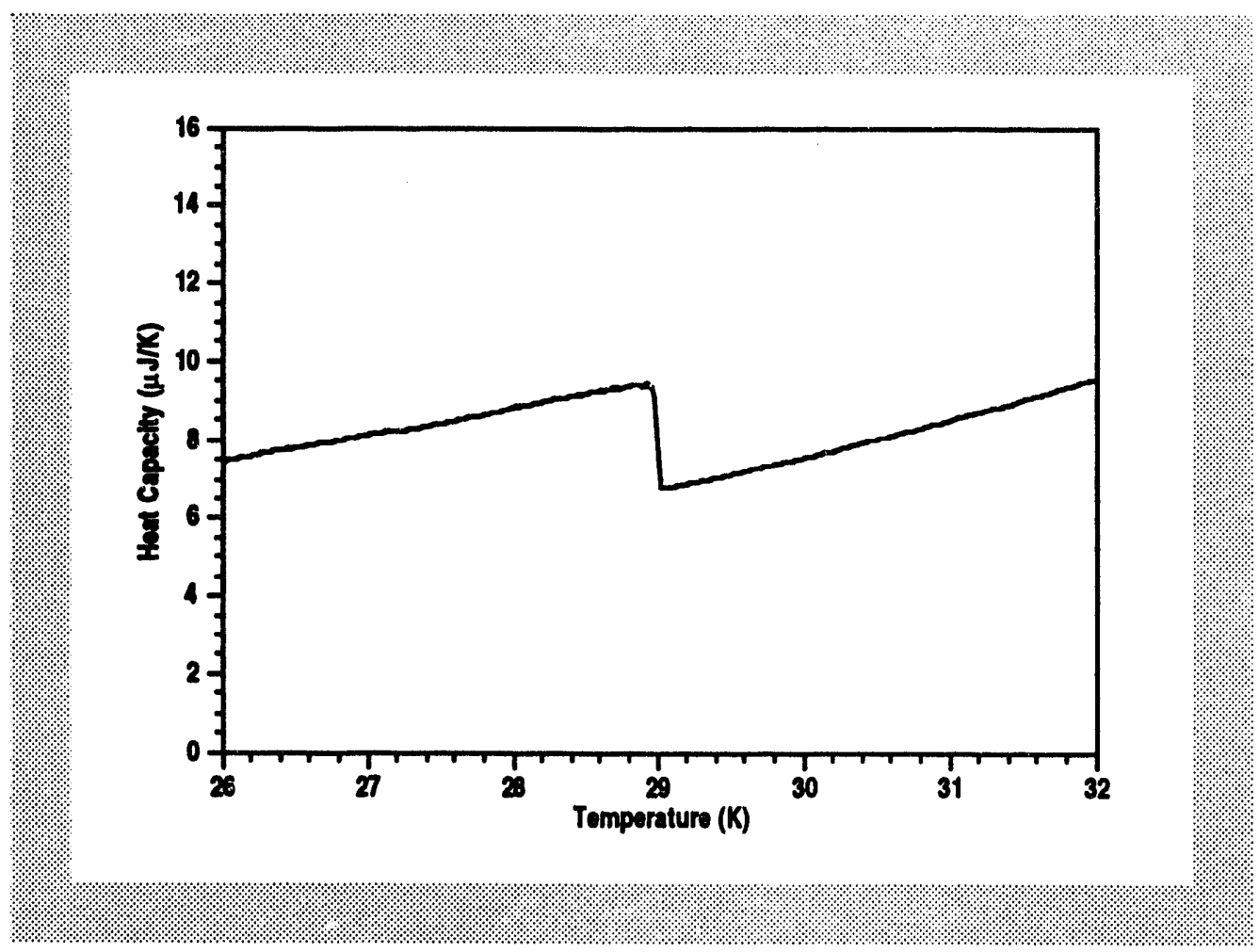

Fig. 3-17. Measured heat capacity of shell and calorimeter.

\subsubsection{SPHERICAL INTERFEROMETRY}

Atomic Force Microscopy (AFM) and phase shift interferometry are two metrology methods possessing the angstrom level resolution capability we need to characterize ICF capsule surfaces. AFM studies have been conducted at LLNL where a technique has been developed in which a series of traces around the circumference of a capsule are used to measure surface roughness. Since the AFM does a series of traces, its field of view is restricted. In contrast, phase shift interferometry provides a means of doing areal measurements on sections of a capsule. Significantly more information can be collected, and, in principle, a $4 \pi$ mapping of a capsule can be produced by patching the individual sections together.

Interferometry has been used to characterize spherical objects. Subaperture optical testing techniques have been applied to centimeter-size balls to determine their sphericity [Ref. 3-16]. This involved piecing together a set of phase maps, taken with an interferometer, to generate the phase map of the complete surface. This complete phase map was fit to a series of spherical harmonics, $Y_{\ell, \mathrm{m}}(\theta, \phi)$, for $\ell \leq 6$. Features corresponding to higher order 
$\ell$-modes were not used. Our study looked into using a phase shift WYKO interferometer at LANL to perform surface roughness measurements. A test capsule was measured using both this interferometer and the AFM at LLNL, and then the results were compared. Surface roughness information from the higher order surface features was extracted in terms of the Power Spectral Density (PSD).

Figure 3-18 shows a schematic of the experimental setup. Different sections of a postmounted capsule were measured by rotating the stalk in its mount. With the $f / 1.5$ lens the angle subtended on the capsule was about $37^{\circ}$. A complete $360^{\circ}$ mapping around the circumference of the capsule required a minimum of ten sections $\left(37^{\circ} \times 10=370^{\circ}\right)$. Therefore, the lowest $\ell$-mode we can expect to see in a patch is $\ell=10$. The resolution of the system was difficult to determine and we did not quantify it. However, after studying the data from repeated measurements on the same section and from overlapping sections, we believe we can see features with full-widths at half-maximum of three to four pixels

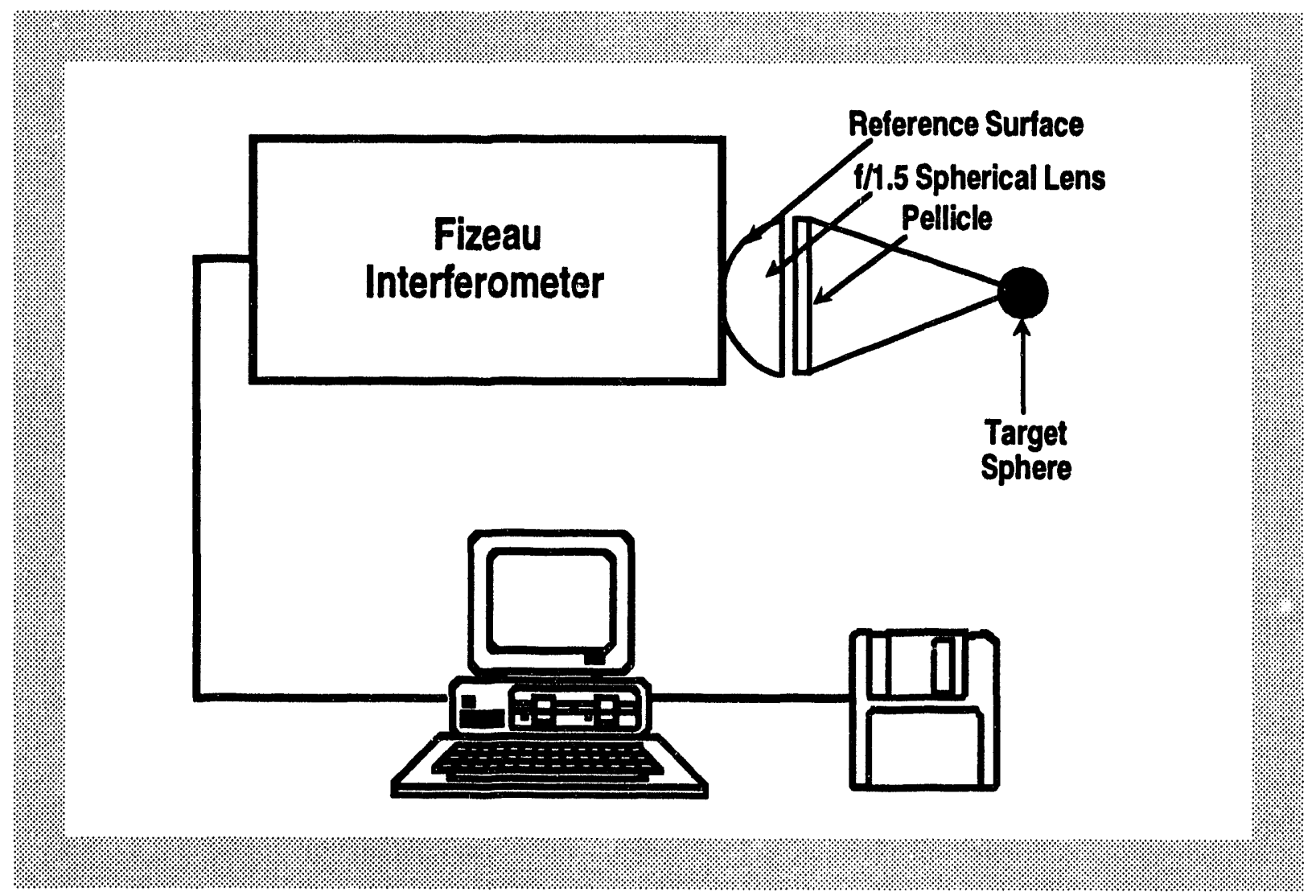

Fig. 3-18. Schematic of the interferometric setup. 
(10 milliradians) and heights of $20 \AA$. Repeated measurements on the same sphere section were repeatable to an $\mathrm{rms}$ of $\lambda / 1600$, where $\lambda=633 \mathrm{~nm}$. The corresponding instrument specifications were listed as: rms repeatability of $\lambda / 2000$ and peak-to-valley repeatability of $\lambda / 300$.

We used the following variation on the basic sub-aperture testing procedure to generate the PSD:

1. Measure the optical path differences (phase maps) on small areas (sub-apertures) at known locations on the large surface (full aperture).

2. Fit the data to a set of spherical harmonics to describe each sub-aperture's shape.

3. Subtract the alignment terms (tip, tilt, piston, and focus) from each sub-aperture set of spherical harmonics so that only those terms relating to surface shape remain.

4. Fit together line-outs from the corrected phase maps and calculate the PSD using a Fast Fourier Transform (FFT).

Both AFM and interferometer measurements were performed on an aluminum coated $506 \mu \mathrm{m}$ capsule. The procedure to calculate the PSD from the interferometer data was analogous to the procedure used for the AFM. The PSD $\ell$-mode information generated from both methods are shown in Figs. 3-19 and 3-20. A direct comparison of the figures shows very similar results between modes ten and a few hundred. The only noticeable differences between the figures are the high- and low-order mode cutoffs. The cutoffs for the interferometer measurements are determined by the positioning of the capsule for the measurements and by the number of data points used in the FFT. Since the capsule was not held in a precision mount, there is an ambiguity in positioning needed to calculate $\ell$-modes less than ten. The AFM measurements used 4096 data points while there were 2048 data points from the interferometer measurements.

The key to using this interferometry technique is having a suitably designed interferometer. The WYKO interferometer demonstrated the suitability of this technique but significant hurdles must be crossed before spherical interferometry can be implemented as a standard characterization procedure. The WYKO interferometer was not designed to do measurements on small objects. With its six inch aperture, it was designed to measure large 


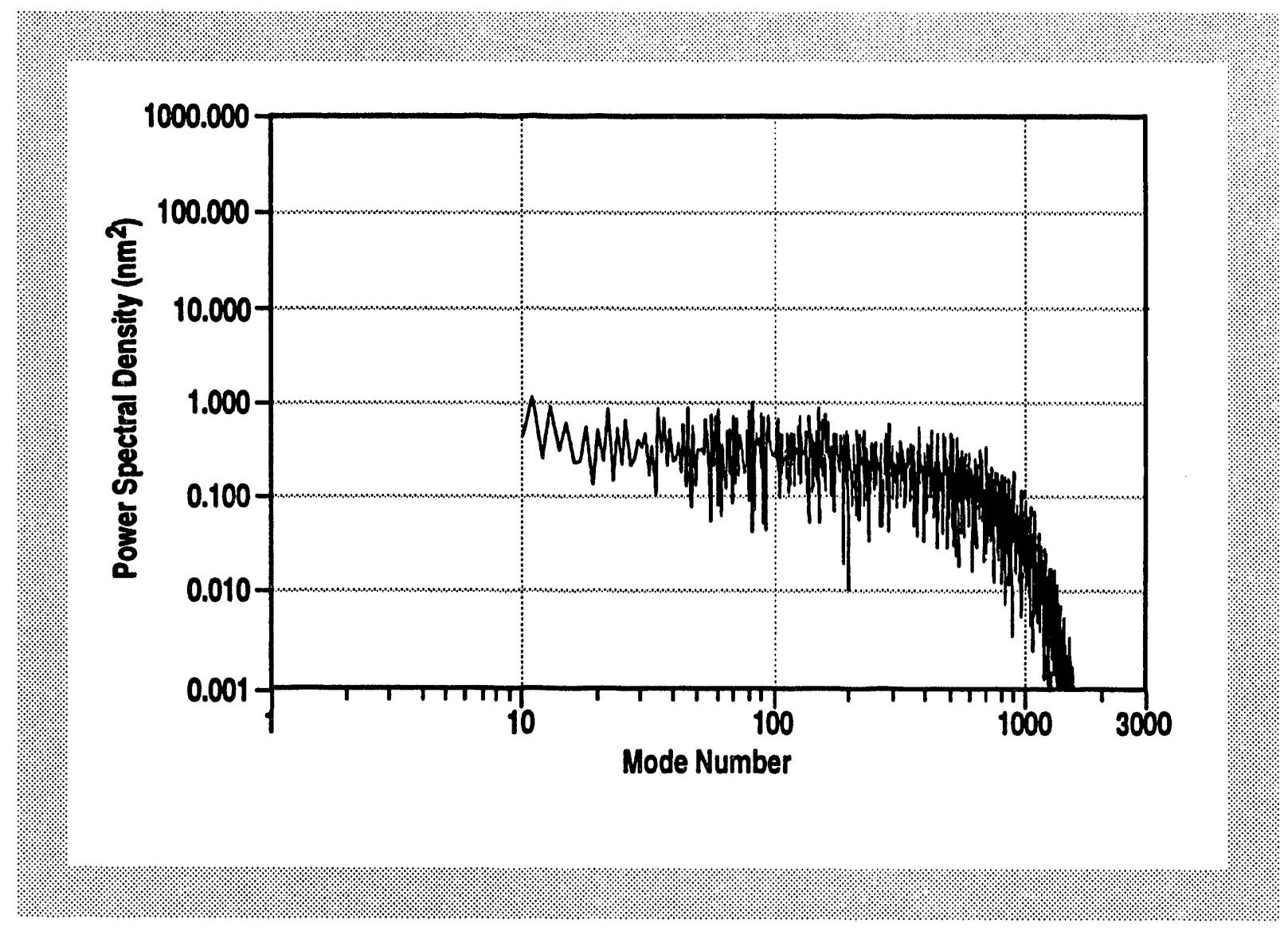

Fig. 3-19. Power spectral density from the interferometer measurements.

optics. The major disadvantage in the use of this instrument is that measurements were restricted to opaque capsules. Transparent capsules were tested initially, but reflections from multiple surfaces generated complicated fringe patterns that the interferometer either could not handle or resulted in residual fringe patterns remaining in the phase maps. A procedure would need to be developed for removing reflections not coming from the surface of interest. On subsequent experiments, only opaque objects were used. Also, a rotation protocol will be required so that the capsule can be rotated in an indexed fashion, especially if a $4 \pi$ map is required. We matched overlapping sections by hand. This was a labor intensive way of connecting data sections and would be particularly cumbersome to align for sections off a circumferential trace. A report was written describing this work in more detail [Ref. 3-17].

For further information, please contact Dr. J. Larsen (CAS) or Dr. D. Bittner (WJSA). 


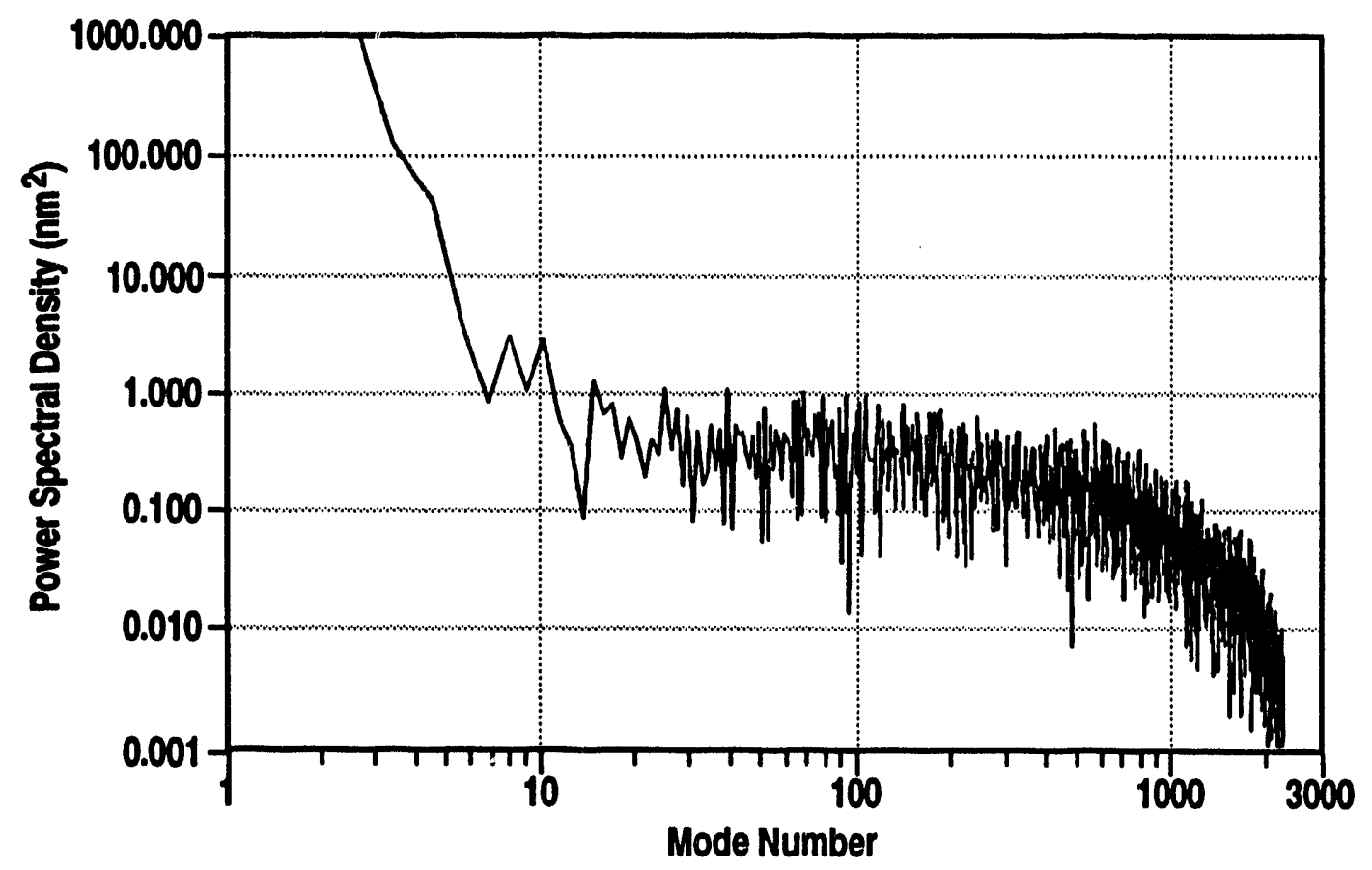

Fig. 3-20. Power spectral density from the AFM measurements.

\subsection{REFERENCES}

[3-1] Martin, A.J., Simms, R.J., and Jacobs, R.B., J. Vac. Sci. Technol. A6, (1988) 1885.

[3-2] Hoffer, J.K., and Foreman, L.R., Phys. Rev. Lett. 60, (1988) 1310.

[3-3] Hoffer, J.K., and Foreman, L.R., Simpson, J.D., Pattinson, T.R., Physica B 165 \& 166, (1990) 163.

[3-4] Simpson, J.D., Hoffer, J.K., and Foreman, L.R., Fusion Technology 21, Part 2, (March 1992), p. 330.

[3-5] Simpson, J.D., "1992 ICF Annual Report," General Atomics, p. 3.1.

[3-6] Collins, G.W., et al., "Solid Hydrogen Surfaces," ICF Quarterly Report, Vol. 3 (2) Lawrence Livermore National Laboratory (UCRL-LR-105821-93-2), p. 81. 
[3-7] Image Acquisition and Analysis software provided by E.R. Mapoles of Lawrence Livermore National Laboratory.

[3-8] Hoffer, J.K., and Foreman, L.R., Mapoles, E.R., and Simpson, J.D., "Forming a 'Perfectly' Uniform Shell of Solid DT Fusion Fuel by the Beta-Layering Process," presented at the IAEA $14^{\text {th }}$ International Conference on Plasma Physics and Controlled Nuclear Fusion, Wurzburg, Germany, September 30, 1992, paper No. IAEA-CN-56/G-3-4, to be published in Nuclear Fusion.

[3-9] Hoffer, J.K., and Foreman, L.R., Phys. Rev. Lett. 60 (1988) 1310.

[3-10] Souers, P. C., "Hydrogen Properties for Fusion Energy," 1st ed., University of California Press, Berkeley, California (1986).

[3-11] We wish to thank W. Earl (LANL) for the use of his spectrometers and Y.W. Kim (Univ. of New Mexico) for sharing his NMR and NMR probe design expertise.

[3-12] Annual Report to the U. S. DOE (GA-A21203, UC-712), p. 3-8, (1993).

[3-13] Sanchez, J.J. and Upadhye, R.S., "A Non-Destructive Method for Measuring the Fill Pressure and Permeability of Plastic Shells," Lawrence Livermore National Laboratory ICF Quarterly Report, Vol. 1, 1 (1990).

[3-14] Sullivan, P.F. and Seidel, G., Physical Review 173, (1968) 679; Kenny, T.W. and Richards, P.L., Rev. Sci. Instrum. 61, (1990) 822.

[3-15] White, G.K., "Experimental Techniques in Low-Temperature Physics," Oxford University Press, New York, (1987) p. 174.

[3-16] Day, R.D., and Lawrence, G.N., Precision Engineering 11, (1989) 3.

[3-17] Bittner, D.B. and Larsen, J., "Surface Characterization by Interferometry," Report to DOE under contract No. DE-AC03-91SF18601, November 1993. 


\section{SECTION 4}

\section{MICROENCAPSULATED SHELLS}




\section{MICROENCAPSULATED SHELLS}

An important goal of our work is to develop improved ICF target fabrication techniques that will facilitate more efficient production of targets. Production of polymer shells is an area targeted for improvement.

\subsection{INTRODUCTION}

Microencapsulation is one of the two common techniques (the other being drop tower) used to produce polymer shells for ICF target fabrication. Microencapsulated shells have the advantage of being more concentric than shells produced in drop towers. It also appears that the size of microencapsulated shells may not be as limited as the size of drop tower shells. However, the surface finish and wall integrity (presence of vacuoles) of microencapsulated shells are not as good as drop tower shells.

Controlled-mass microencapsulation is one of the most promising approaches for polymer shell production. Controlled-mass microencapsulation uses a triple-orifice droplet generator to form polymer shells with a predictable and controllable size and wall thickness, potentially eliminating tedious sorting necessary with either the drop tower or the complex emulsion technique.

The objective of this task was to improve the quality of shells produced by the controlled-mass microencapsulation technique. This was accomplished by gaining an understanding the fundamental shell formation process, conducting appropriate experiments, and engineering improved equipment. Modeling of the shell solidification process in the form of a finite element computer model gave us a fundamental understanding of how physical phenomena affect processing. We used statistical methods to optimize the organic solution composition and improved the design of the controlled-mass system to enhance the quality of product shells. 


\subsection{POLYMER SCIENCE}

Polymer science issues are critical in the production of polymer shells using the microencapsulation technique.

\subsubsection{INTRODUCTION}

The production of microencapsulated polymer shells is a very complicated process. Without a fundamental understanding of the underlying physics of microencapsulated shell production, it is impossible to produce multi-millimeter, target quality shells without performing a vast number of time consuming experiments. Polymer science, mass transfer, and thermodynamics are all important areas of knowledge which will contribute to the production of polymer shells by allowing the appropriate experimental phase space to be intelligently identified. Our area of emphasis during FY93 has been the building of a mass transfer model which predicts the solidification behavior of shells formed by the tripleorifice droplet generator. We also completed phase diagram measurements of the mixture of polystyrene $(\mathrm{MW}=152,000)$, methylethyl ketone $(\mathrm{MEK})$, and water.

\subsubsection{MASS TRANSFER MODELING}

The mass transfer process during solidification is the most important aspect of shell formation. With this in mind, we chose to concentrate our efforts in this area during FY93. The goal of the model is to predict shell size and wall thickness as a function of time, the total time for solidification, and most importantly, the composition profile within the wall as a function of time. The composition of the shell wall is used in conjunction with phase equilibrium data to predict the formation of vacuoles (voids within the shell wall).

As a first step toward producing the model, we derived the governing equations through unsteady-state mass balances and the proper choice of constitutive relationships. We assumed Fickian Diffusion with transport-induced convection as the mechanism of composition evolution within the shell wall. Therefore, the flux of solvent $A\left(N_{A}\right)$ at any point $r$ within the wall is described by:

$$
N_{A}=-D_{A} \rho \nabla w_{A}+V_{I} \rho w_{A}
$$

where $D_{A}$ is the diffusivity of component $A, \rho$ is the mixture density, $w_{A}$ is its weight fraction, and $V_{r}$ is the volume average velocity within the wall, all evaluated at $r$. Applying 
a mass balance on the shell and assuming radial symmetry, we arrived at the following governing equation, valid for each component in the shell wall.

$$
\rho\left(\dot{w}_{A}+v_{r} \frac{\partial w_{A}}{\partial r}\right)=\frac{1}{r^{2}} \frac{\partial}{\partial r}\left(r^{2} \rho D_{A} \frac{\partial w_{A}}{\partial r}\right)
$$

$\left.\dot{\mathrm{w}}_{\mathrm{A}} \mathrm{j}\right\}$ the time derivative of the weight fraction of component $\mathrm{A}$. The constitutive equation used for the diffusivity is the Fujita-Doolittle equation given by:

$$
\log \frac{D}{D_{0}}=\frac{v_{s}}{A+B v_{s}}
$$

where $D$ is the concentration dependent diffusivity, $D_{0}$ is the diffusivity at infinite dilution, $v_{s}$ is the volume fraction of solvent, and $A$ and $B$ are parameters dependent on temperature and chemical structure.

To properly solve this problem, two boundary conditions and an initial condition are required. The first boundary condition is that no mass transfer takes place at the inner interface. This implies that the inside diameter of the shell is fixed throughout the solidification process. This assumption is accurate as long as the components in each of the walls are relatively immiscible, which is generally the case in microencapsulation. In the future, this boundary condition will be relaxed to confirm its validity. At the outer shell boundary, mass transfer takes place due to the concentration difference between the boundary layer at the outside of the shell wall and the bulk fluid surrounding the shell, assumed to be zero. The concentration at the boundary layer is given by Henry's Law. A mass transfer coefficient is used to determine the magnitude of the flux generated. The initial condition is a uniform composition throughout the shell wall.

Using the finite element method, we solved the problem for a polymer shell with one solvent being removed via diffusion. Our results have already provided much insight into the solidification process and have allowed us to visualize it graphically. Figure 4-1 is a graph of percent solvent versus time for three simulations with different solvents. Notice that benzene is predicted to require six days for solidification, while methyl ethyl ketone (MEK) needs only 1.78 hours. Figure 4-2 plots the solvent concentration at the outer edge of the shell as a function of radius. Benzene is shown to have a much higher boundary solvent concentration as the shell solidifies, so the profile through the wall is much more flat than the other two solvents throughout the process. Finally, Fig. 4-3 ties together the 
modeling and the polymer solution thermodynamics (see Section 4.2.3). It shows the concentration profile in the wall, the outer radius and wall thickness, the percent of initial solvent remaining, and a phase diagram with the current wall composition cross-plotted. If the shell wall composition is within the two phase region of the phase diagram, vacuoles are predicted to form. Using the model to simulate microencapsulation runs, including composition and processing variables, vacuole-free processing methods may be found.

\subsubsection{POLYMER SOLUTION THERMODYNAMICS}

Polymer solution thermodynamics is important because polymer phase separation is believed to be the mechanism of vacuole formation. Vacuoles are defects in the shell wall which can disqualify the shell. Phase equilibrium measurements of 152,000 molecular weight polystyrene dissolved in MEK and water were completed. Figure 4-4 shows cloud point data for 152,000 as well as 106,000 molecular weight polystyrene. We used this data

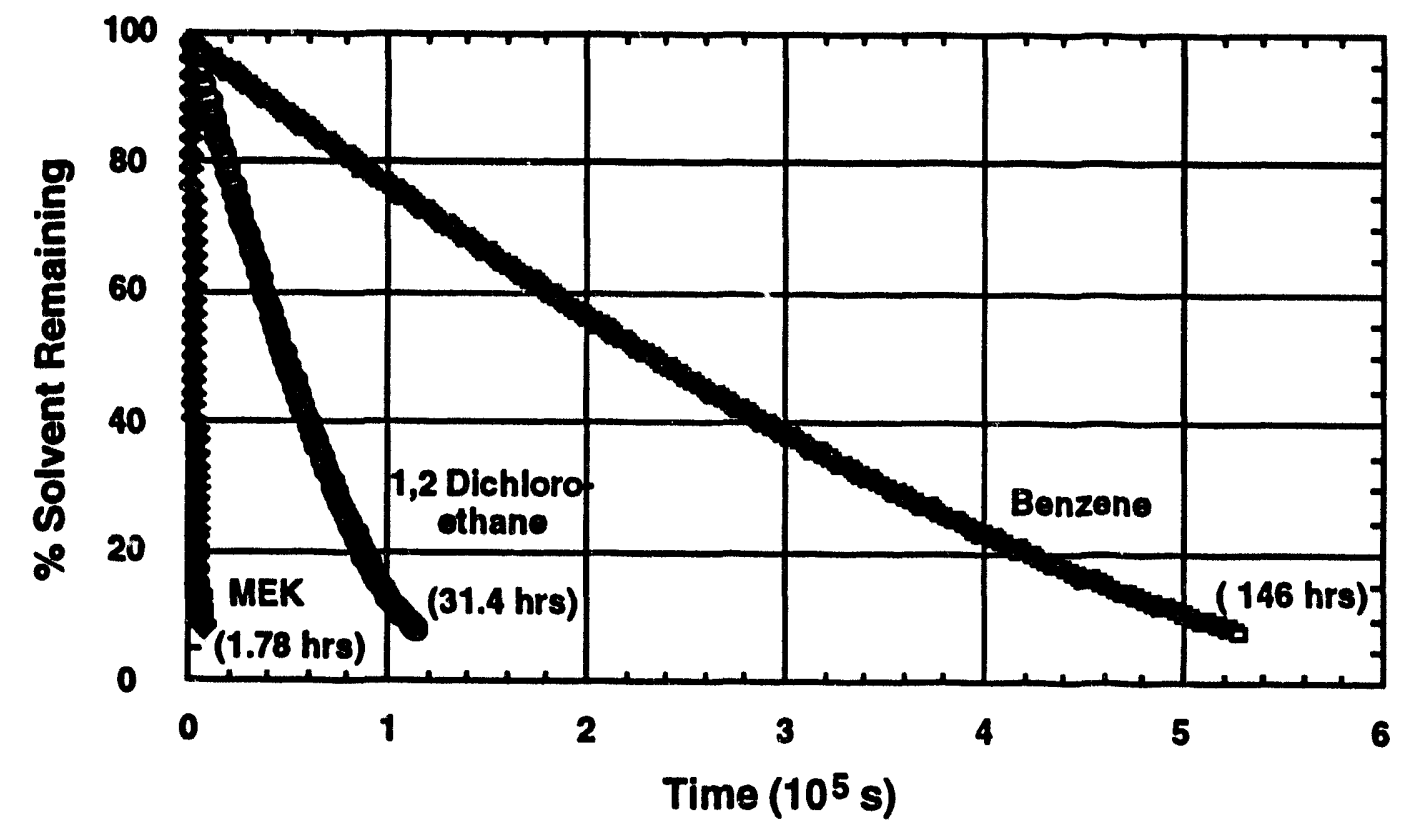

Fig. 4-1. Percentage of Initial solvent remaining versus time. The simulation initial outside diameter was $1 \mathrm{~mm}$ and the wall thickness was $100 \mu \mathrm{m}$. The simulation was stopped when only 10 percent of initial solvent remained. 


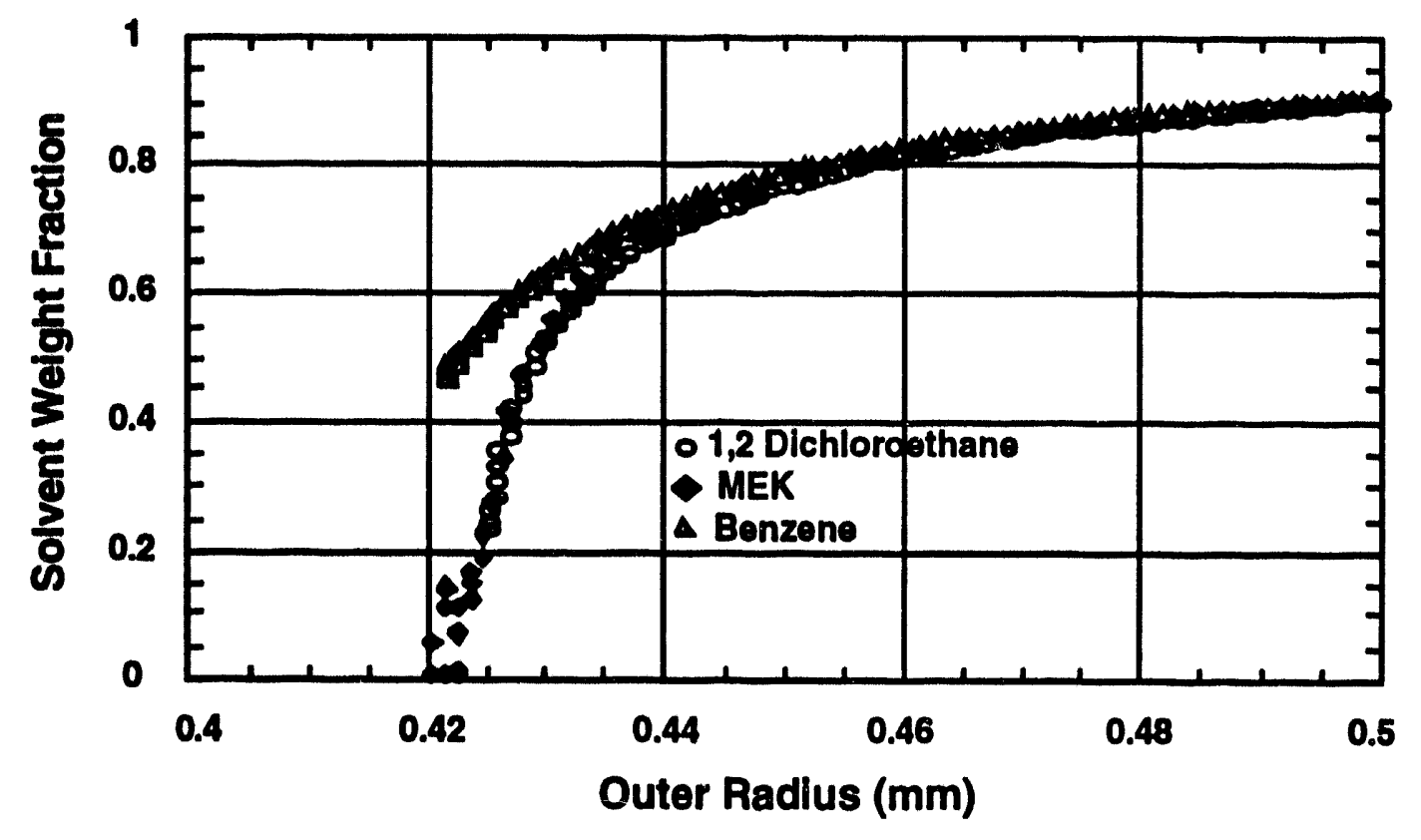

Fig. 4-2. Solvent concentration at the outer edge of the shell as a function of outer radius.

to generate equilibrium phase diagrams. At temperatures above the curve, the polymer solution is clear. At temperatures below the curve, the mixture is phase separated, becomes cloudy, and the resulting shells contain vacuoles.

For additional information, please contact Dr. D. Nelson or Dr. D. Soane (STI).

\subsection{STATISTICAL PROCESS ANALYSIS}

The statistical process analysis was a purely empirical study of the solvent-polymer system used for microencapsulation. The analysis had two purposes. First, it complimented the theoretical modeling. It supplied actual results for a variety of conditions that may be used as reference points for the theoretical model. Second, it simplified the controlled-mass microencapsulation development by separating the chemical processes from the mechanical ones (see Section 4.4). We used the "complex emulsion" microencapsulation technique to ensure that we would see the full range of effects of the solvent system. 


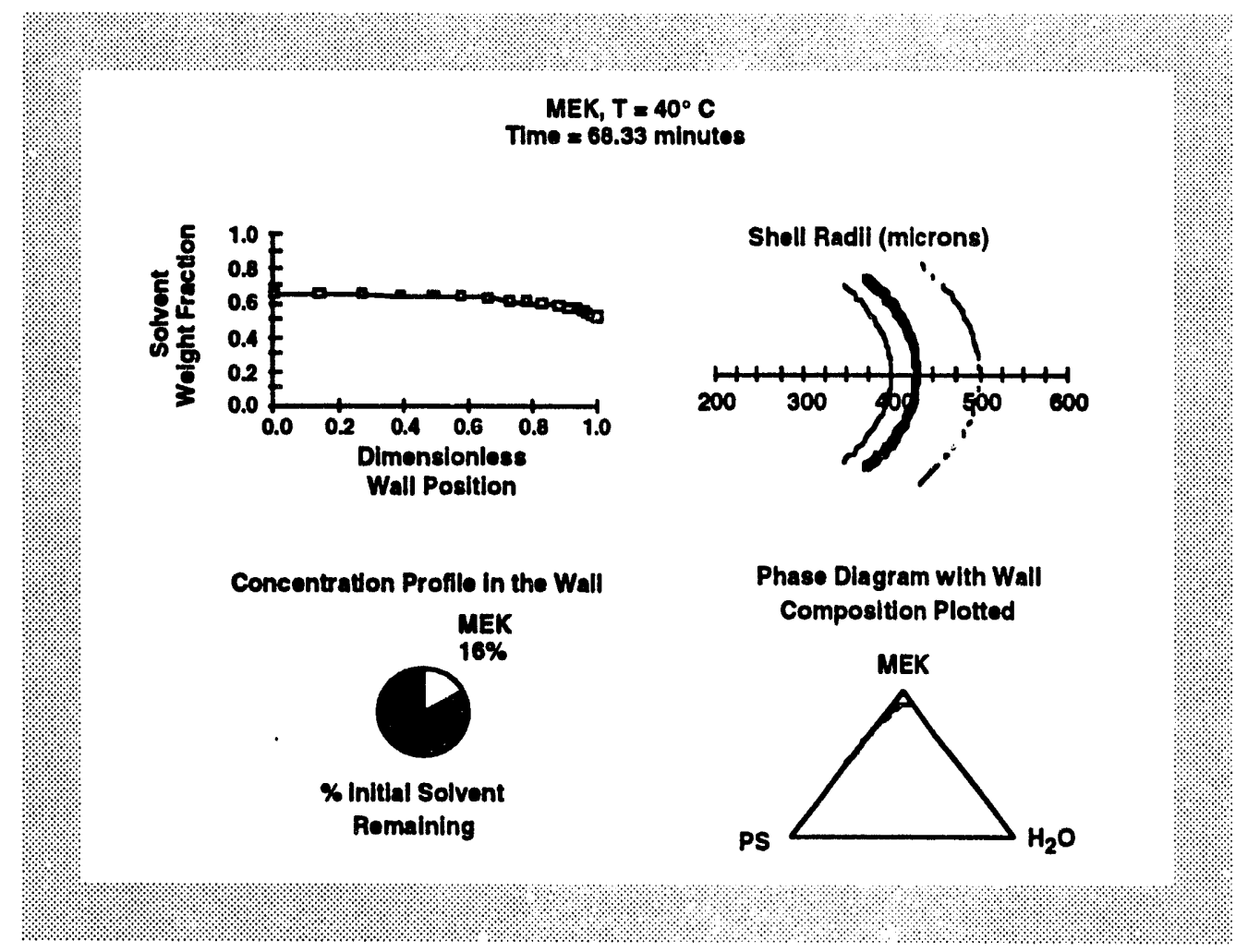

Fig. 4-3. Summary of all results of the modeling and how it is incorporated with phase equilibrium data. The phase diagram in the lower right hand corner has the composition profile in the shell wall crossplotted. If the wall composition is in the two-phase region,

This analysis was a direct result of the screening experiments done last year. The screening experiments demonstrated that multi-millimeter microencapsulated spheres were possible if a water soluble solvent was added to the typical solvent system. This discovery lead to the search for a blend of solvents capable of producing vacuole-free, multimillimeter spheres.

Many of the basic statistical techniques are unchanged from last year. The trials were randomized and the shell batches were characterized as a set. Empirical models were generated, checked for appropriate fit, and confirmed by running new trials.

\subsubsection{DESIGN}

The first step was to identify the number of process components and any restrictions. Obviously, the typical solvents, benzene and dichloroethane, would be included. We also 


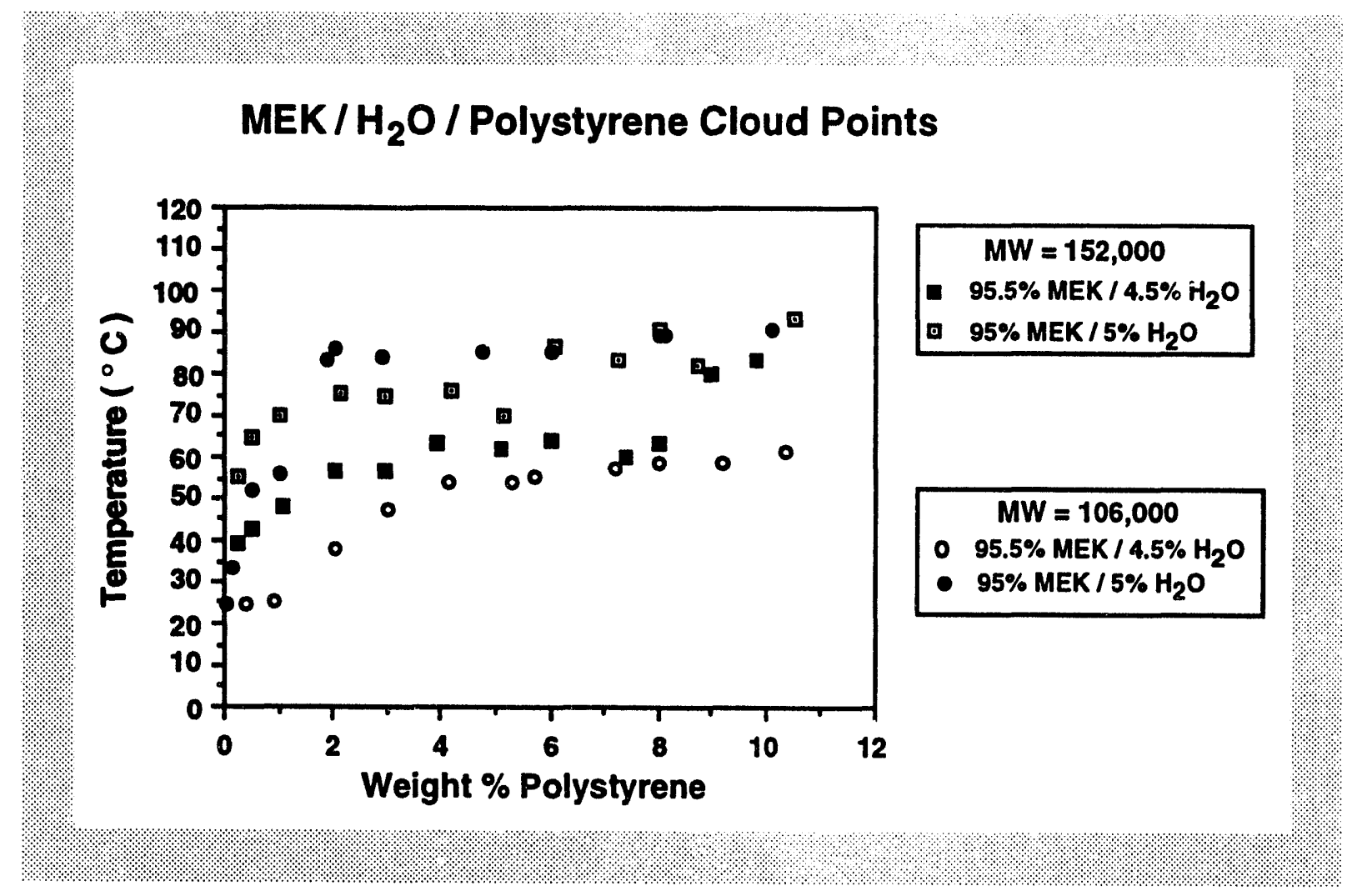

Fig. 4-4. Data from Thermal Optical Analysis experiments comparing the effect of molecular weight on phase behavior. The cloud point is the temperature where the polymer solution becomes cloudy.

chose MEK as it produced larger spheres. Unfortunately, MEK also produced spheres of lower uniformity and increased vacuole concentration. We added styrene in an attempt to overcome these defects. Styrene represents the antithesis of MEK in this system. MEK increases water solubility, styrene lowers it. MEK leaves the system quickly, styrene leaves very slowly.

Our hope was to create a solvent system that would change dramatically over time. Initially, the solvent system is MEK rich. MEK stabilizes the water-solvent interface just as a surfactant would, but with the advantage of leaving no chemical traces. Thus, it promotes large sphere formation. The MEK leaves quickly, but the large size is still stable as the viscosity of the solvent system increases dramatically as the polymer becomes more concentrated. Unfortunately, this higher viscosity also stabilizes wall non-uniformities and entrapment of water within the solvent phase. Styrene, however, is still in the solvent system. Styrene's long removal time allows for a long period of soft polymer for the 
removal of the small defects without losing the large sphere size. For this model to work, the styrene concentration has to be small compared to the MEK concentration.

Having selected four solvents, we identified the process constraints. We conducted ranging experiments and demonstrated that spheres could be produced with solvent systems of 0 to 90 volume \% benzene, 0 to 75 volume \% MEK, and 0 to 90 volume \% dichloroethane. We added polystyrene and styrene to the solvent blends by weight percent, $10 \%$ being the upper bound for both.

We could now identify an appropriate design. A mixture design was used, as it is designed to sum the fractions of the components to one. Data points were selected for their ability to cover the parameter space and to confirm the assumption of reproducibility throughout the design. (We used the computer program ECHIP for data point selection and result analysis.) We further simplified the parameter space by setting benzene, 1,2dicholorethane, and MEK as the three primary components and treated styrene and polystyrene as additives. Thus, the primary components form a three-axis system while the additives are treated as off-axis variables. This three-axis system can be easily visualized and graphed as shown in Fig. 4-5.

\subsubsection{RESULTS}

The primary goal of this analysis was to produce millimeter-size, vacuole-free, polymer spheres, but we actually studied six results: mean diameter, mean wall thickness, mean aspect ratio, uniformity, vacuole concentration, and stability of the microencapsulated preform. (In our efforts to produce large vacuole-free shells, we did not wish to amplify other defects.) We tested the results for correlation, then plotted them on the z-axis above the three solvents (see Fig. 4-6). In this manner, we could study the effects versus solvents. We had three critical findings. First, the largest diameters were predicted for the $100 \%$ dichloroethane solvent system, and the lowest vacuole concentrations were predicted for the $100 \%$ methyl ethyl ketone solutions. Obviously, these two solvent systems are mutually exclusive. Second, the graphs were sloped as they approached the maxima. This indicates that both properties could be enhanced further, if we could extend the axis. Third, benzene is not contributing to either maxima. It is not an ideal solvent.

This left us with the problem of extending the analysis beyond the given solvents. We chose not to repeat the experiments with other solvents, but to re-analyze the results by 


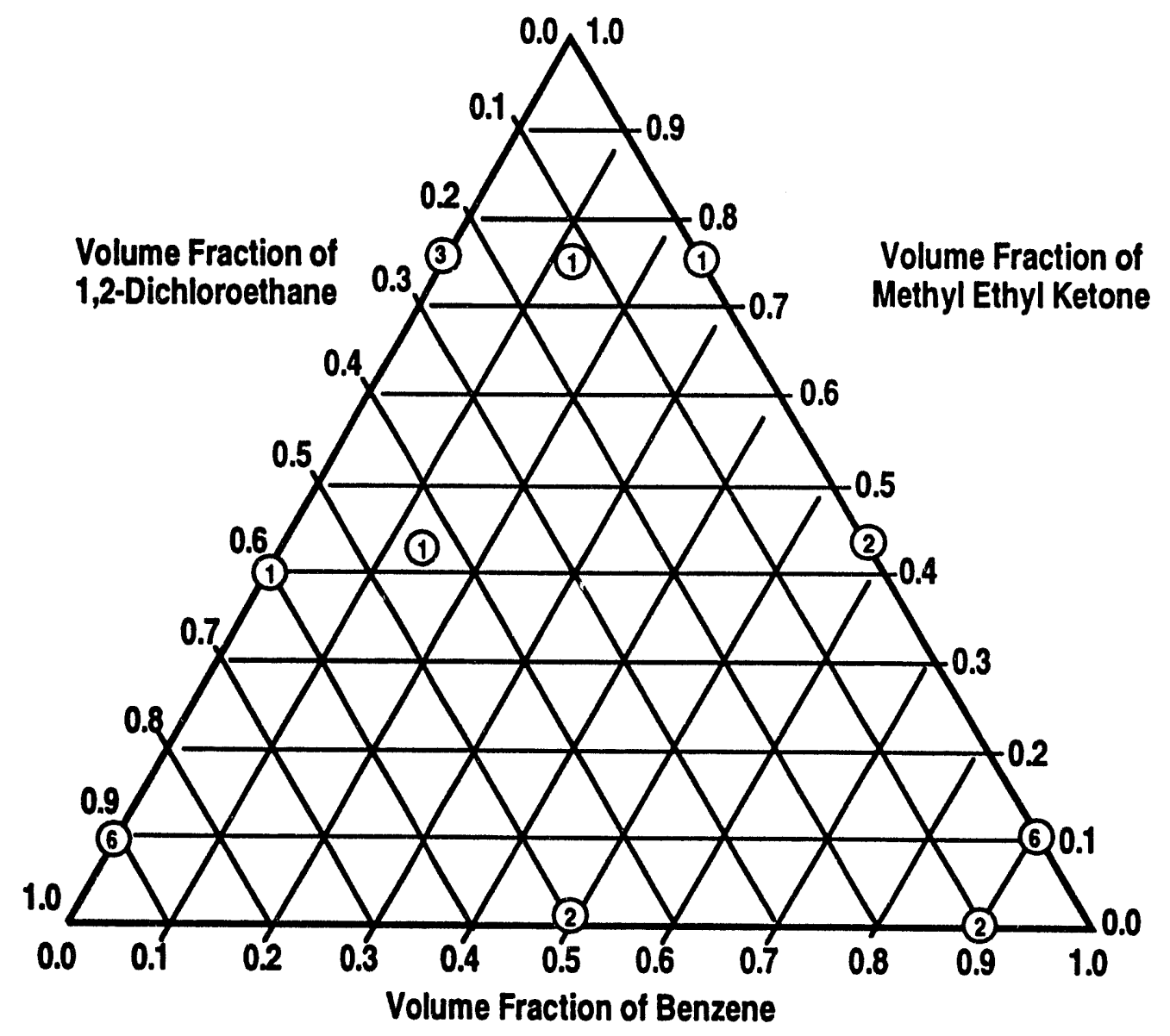

Fig. 4-5. Graph showing the three-axis solvent system. Each side of the triangle is a label axis for a solvent. The values run at a 60 degree angle from the label axis. At any point within the triangle, the fractions will sum to one. Data points are indicated by ovals. The number within the oval indicates the number of trials at that point.

using the solubility interactions of the solvents involved. Solubility parameters (represented by $\delta$ ) are estimates of three types of interactions that a solvent molecule exhibits: polar, dispersive, and hydrogen bonding. We chose the parameter system as determined by Hansen [Ref. 4-1] for its thoroughness of derivation. The polar component, $\delta_{p}$, is calculated from the dipole moment of the molecule. The dispersion component, $\delta_{d}$, is calculated by comparison of interactions between similar molecules with and without polar components. 


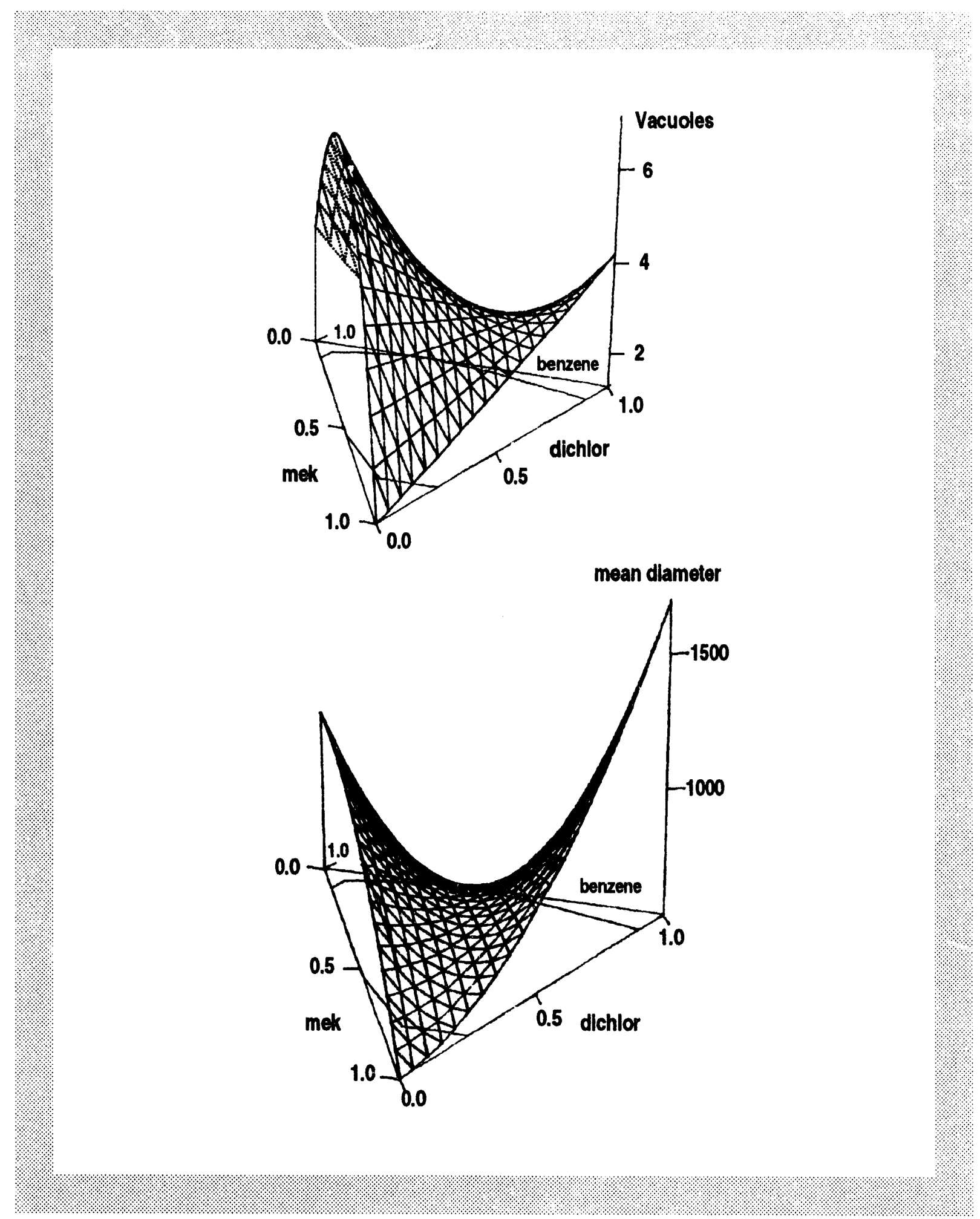

Fig. 4-6. Two graphs indicating the results for mean diameter and vacuole concentration. The solvents vary along the sides of the triangles, the responses vary along the z-axis. 
The hydrogen bonding component, $\delta_{\mathrm{h}}$, is calculated from the IR analysis of the $\mathrm{O}-\mathrm{H}$ stretch of the molecule. These components are usually summed to a total solubility parameter, $\delta_{t}$, using the formula:

$$
\delta_{\mathrm{t}}^{2}=\delta_{\mathrm{p}}^{2}+\delta_{\mathrm{d}}^{2}+\delta_{\mathrm{h}}^{2}
$$

Solubility parameters are derived from analysis of individual solvents, but they are just as appropriate for mixtures. The parameters for mixtures are calculated by multiplying the individual solvent parameter by that solvent's volume percent in the mixture. For our application, the constraints of these parameters are quite different than the constraints of the solvents themselves. Microencapsulation will occur only if the solvents maintain water immiscibility and polystyrene miscibility. This allows only a narrow range of $\delta_{\mathrm{t}}$. To emphasize the individual interactions, we divided $\delta_{\mathrm{p}}^{2}, \delta_{\mathrm{d}}^{2}$ and $\delta_{\mathrm{h}}^{2}$, by $\delta_{\mathrm{t}}^{2}$. This also had the advantage of yielding a three component system with the sum of the components equal to one, as we had with the solvents. Thus, this transformation was also analyzed as a mixture design as shown in Fig. 4-7. This transformation is analogous to one done by Teas, [Ref. 4-2], but maintains the greater numerical precision of the Hansen derivation.

This analysis confirms that large spheres and vacuole-free spheres are produced by two different systems, and the most important factor is the $\delta_{\mathrm{h}}^{2} / \delta_{\mathrm{t}}^{2}$ ratio. The maximum diameter was predicted for spheres with a ratio of 0.218 . The minimum vacuole density was for a ratio of 0.264 . We ran trials with a solvent system of this ratio, and produced vacuole-free spheres, but with diameters of only 250 to $350 \mu \mathrm{m}$.

We do not feel that this has to be the final answer. One solvent system can be made to behave like two because it is exposed to two totally separate water phases. These water phases can be individually modified to change the way the solvent system interacts with them. For example, one water phase could be made more "organic" by adding alcohol; this would make the solvent system act as if its $\delta_{h}$ were lower. Alternatively, a water phase could be made more viscous, thus physically stabilizing the interface but not affecting the solubility interactions. Surfactants can also be used, and they have very specific effects. We can quickly sort between these options because we already have a strong selection guide; we know how we want the solvent system to interact. 


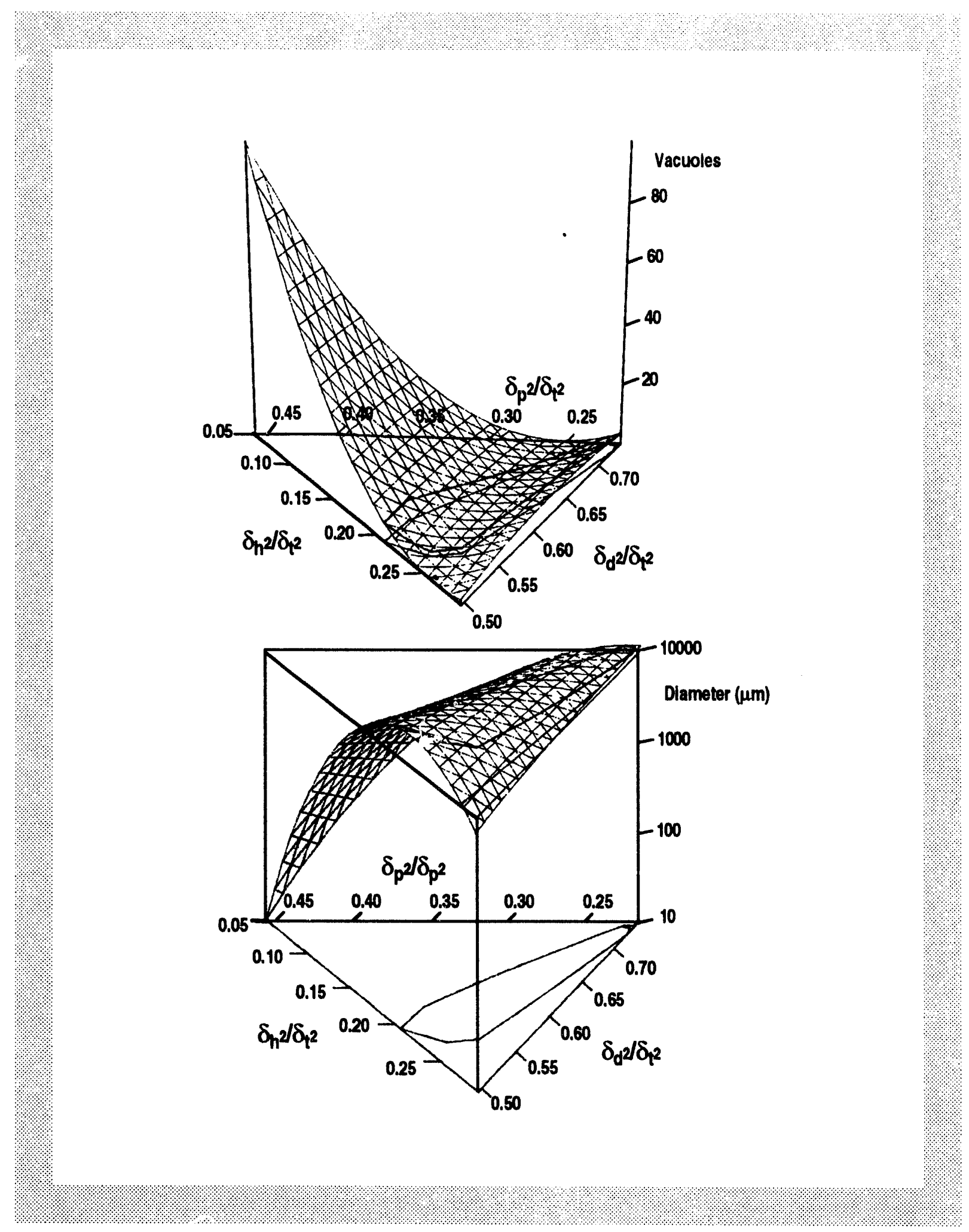

Fig. 4-7. Graphs of mean diameter and vacuole density using the solubility parameter ratios. Negative values of mean diameter indicate that microencapsulation does not occur. 


\subsubsection{CONCLUSION}

Historically, shells for ICF targets had been made by drop tower technology. The shells could be made from either glass or polymer and to specific dimensions. However, the uniformity of the shells became poor as they approached $1 \mathrm{~mm}$ in size, and multi-millimeter shells could not be produced. Microencapsulation offered a simple way to produce high uniformity polymer spheres, but there were vacuoles in the walls. Our research last year demonstrated that microencapsulation is a viable alternative technique, it can produce multi-millimeter shells. This year, we demonstrated that it can also produce vacuole-free shells. Now our goal must be to combine these results and produce the multi-millimeter. vacuole-free, uniform spheres needed for the future.

For additional information, please contact D. Schroen-Carey (WJSA).

\subsection{CONTROLLED-MASS SYSTEM DEVELOPMENT}

Many improvements were made to the controlled-mass system during FY93. These ranged from redesign of the triple-orifice and solidification column to improving the collection of shells from the column and increasing the capacity of the overall system.

\subsubsection{TRIPLE-ORIFICE DROPLET GENERATOR}

At the end of FY92, we had developed a crude, but effective triple-orifice droplet generator. Its major drawback was that it was very difficult to assemble. Axial and radial positioning was a tedious and time consuming process. Our goal early in FY93 was to design a droplet generator that could be easily and quickly assembled by someone with no special knowledge of how the orifice was constructed. The optimal design was to have the whole assembly readily screw together. Simply threading the parts was not adequate, as the tolerances required to achieve concentricity are very difficult to meet simply by using threads. To overcome this, we precisely machined tapered mating surfaces which align each of the orifices to achieve concentricity. Threading of the parts is used only to hold the orifice assembly together. Later in the year, we changed the configuration of the outer orifice to allow more adjustment. Now, we have two orifices that are operable, one with smaller orifices than the other. Figure 4-8 shows the design of the triple orifice we developed in FY93. 


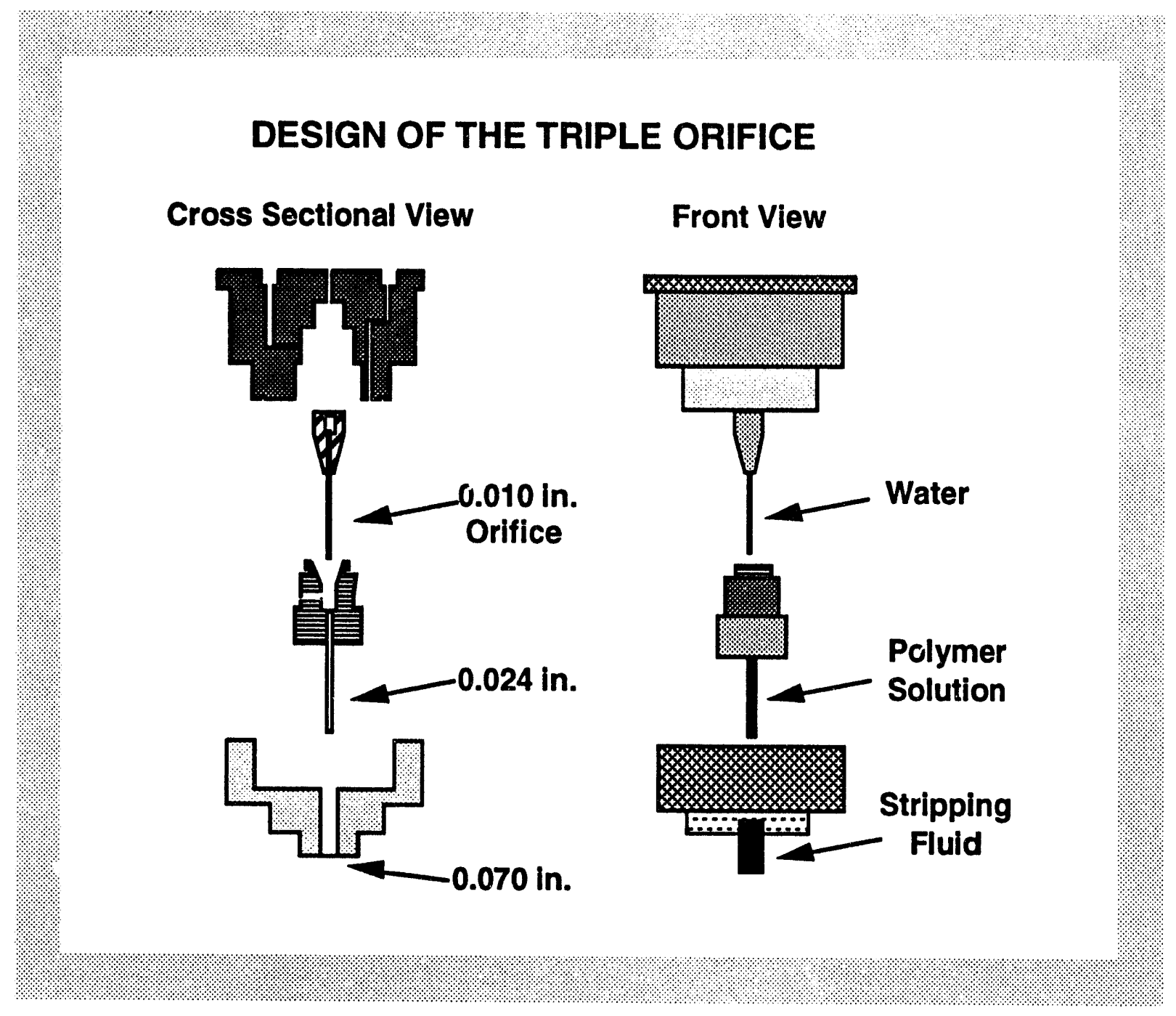

Fig. 4-8. Design of the triple-orifice droplet generator. The three solutions are introduced through fittings in the top of the body.

\subsubsection{SOLIDIFICATION COLUMN DESIGN}

As shells become larger, great difficulties appear during the solidification process. Two problems dominate; suspension and agitation. For much of the first half of FY93, we tested many techniques for column agitation and designs for column configuration. Methods of agitation included: low shear blades, swirling by shaking the entire column, and cones in the bottom of the column with vanes that were rotated to induce mixing. We were not completely satisfied with any of these systems. Finally, we used water jets to agitate the shells, to prevent breakage caused by collisions with mixing blades and shafts. 
Large shells are more prone to settling than smaller shells, so it is important that they not be allowed to fall to the bottom of the solidification vessel. To overcome this tendency, we installed a conical glass column and assembled a recirculating system to provide an upward flow of liquid. Because it is conical in shape, the liquid flow velocity is not zonstant over the length of the column. The shells, which become heavier than water, fall until they reach the point where the upward velocity of liquid is equal to the terminal velocity of the falling shell. The terminal velocity for a falling sphere at low Reynold's Number is given by:

$$
V_{p}=\frac{g D_{p}^{2}\left(\rho_{p}-\rho\right)}{18 \eta}
$$

where $g$ is the acceleration of gravity, $D_{p}$ is the diameter of the sphere, $\eta$ is the viscosity of the fluid, and $\rho_{\mathrm{p}}$ and $\rho$ are the density of the sphere and the fluid respectively.

The shell collection process was also significantly improved during the year. After first installing the conical column, we used a sieve at the entrance of an external tank at the top to collect the shells. The problem with this approach is that the shells were not completely solidified by the time they reached the top of the column. The shells were then damaged by the mesh of the sieve as well as by interparticle collisi uns. We resolved this by inventing the "anti-sieve" and using agitation in the collection vessel. The anti-sieve concept uses a wide-mouth sieve which keeps shells from being carried into the PVA reservoir. The large size keeps the velocity near the exit low, so shells are not dragged into the anti-sieve. In this way, the shells are kept from the mesh, are less likely to collide with one another, and are agitated to maintain their sphericity.

To improve the capacity of the system, which was limited to one run every one to two days, a parallel fluid handling network was assembled. The new arrangement can increase output to as many as three runs per day while only using one conical column. Figure 4-9 shows a schematic diagram of the new arrangement.

\subsubsection{CHARACTERIZATION}

In support of the national ICF program, Soane Technologies, Inc. purchased a Nikon Optiphot 2 microscope with its own funds to perform shell characterization. This system 


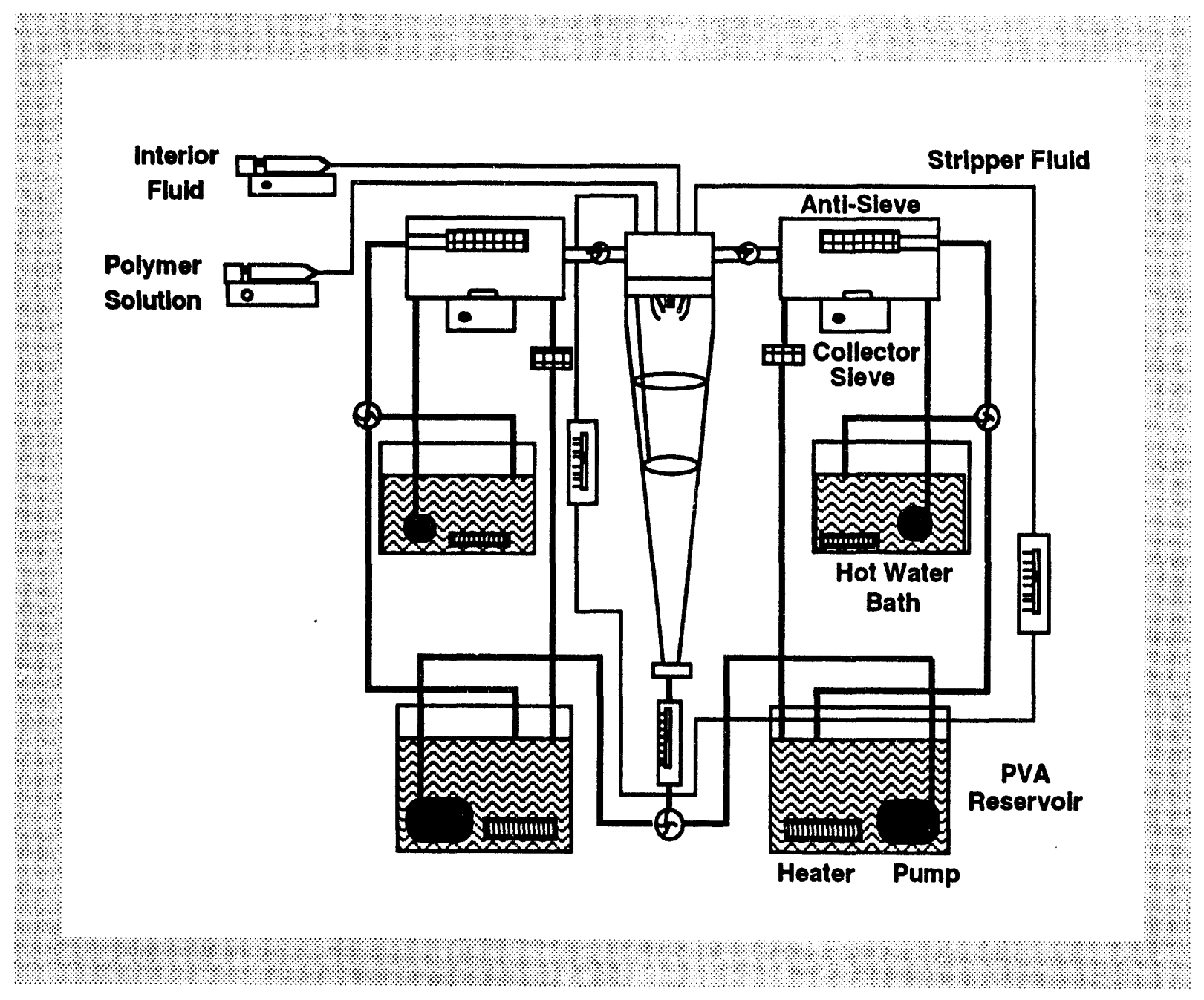

Fig. 4-9. Arrangement of the controlled-mass system which improves output. The new system consists of two parallel fluid handling mechanisms which allow a new run to be performed while other shells are completing solidification.

includes an interference objective to measure shell wall thickness and uniformity as well as a high-power, phase-contrast objective to view defects such as vacuoles. Late in the year, D. Schroen-Carey, of WJSA, assembled a dust shield, an $x-y-z$ translation stage, and a shell rotator for the microscope. This gave us the capability to rapidly acquire feedback on the quality of shells produced with the controlled-mass microencapsulation system.

One of the FY93 achievements we are most proud of is the confirmation of the controlled-mass nature of the triple orifice. Using the Nikon microscope, we found the 


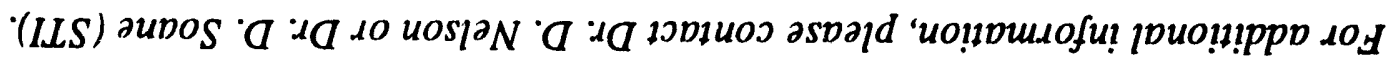

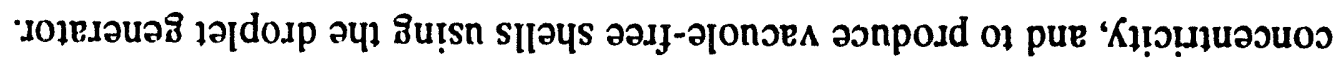

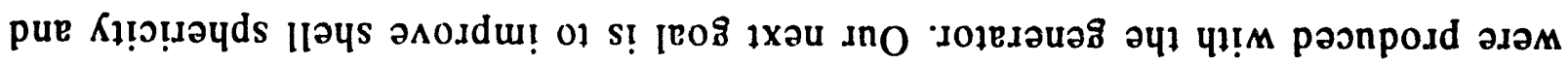

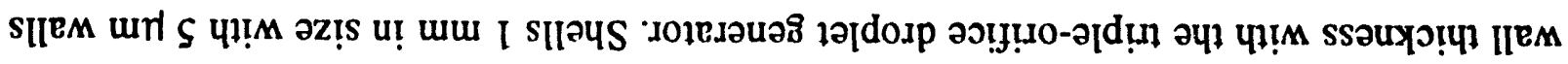

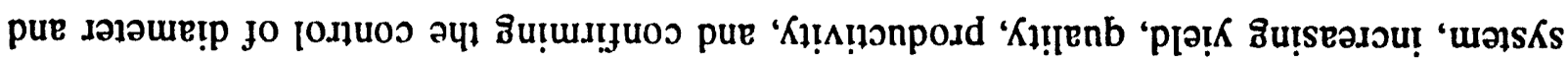

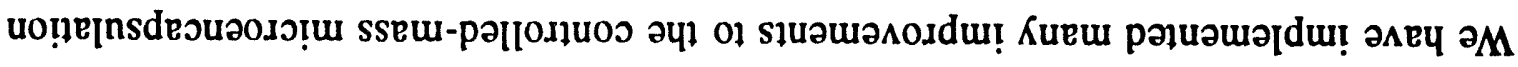

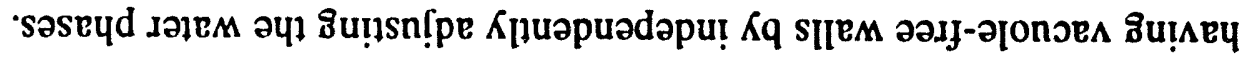

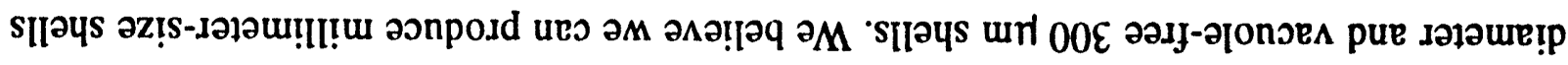

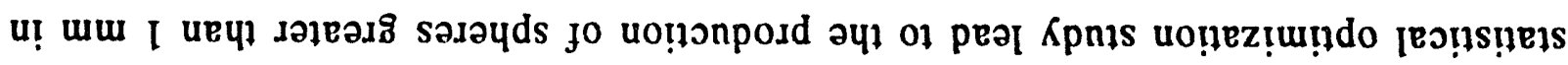

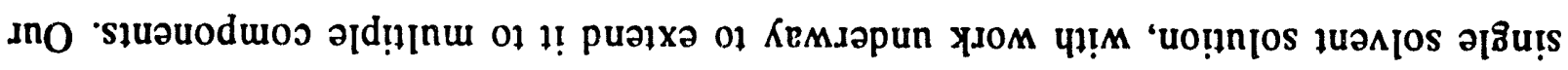

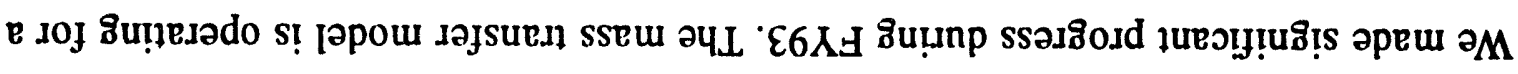

NOISก7ONOJ 's't

\begin{tabular}{|c|c|c|}
\hline \multicolumn{3}{|c|}{ 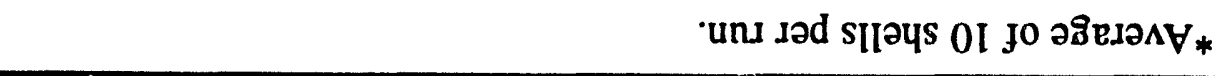 } \\
\hline $9 \circ$ & $t^{\prime} \varepsilon$ & $19^{\circ} 0$ \\
\hline$L \cdot \mathcal{E}$ & $6 \cdot \varepsilon$ & $\varepsilon Z^{*} I$ \\
\hline $\mathcal{\varepsilon} 8$ & $\varepsilon 6$ & $600^{\circ}$ \\
\hline$L^{\prime} \mathcal{E}$ & $9 \cdot \varepsilon$ & $\mathcal{E} I^{*} \mathrm{I}$ \\
\hline $8^{*} \mathcal{E}$ & $L^{\circ} \mathcal{\varepsilon}$ & SI'I \\
\hline 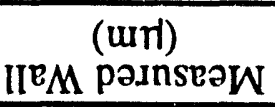 & 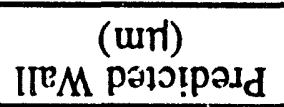 & $\begin{array}{c}\text { (umu) } \\
\text { دəłวure! }\end{array}$ \\
\hline
\end{tabular}

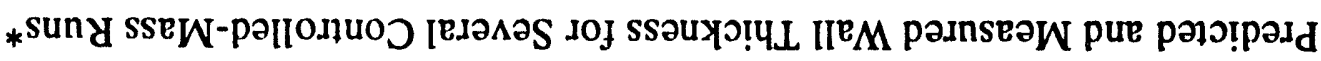

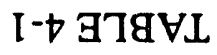

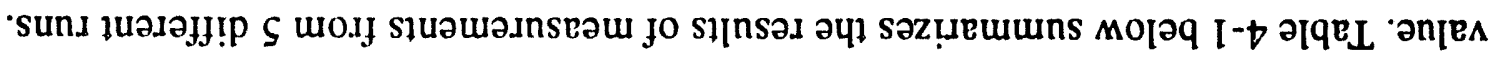

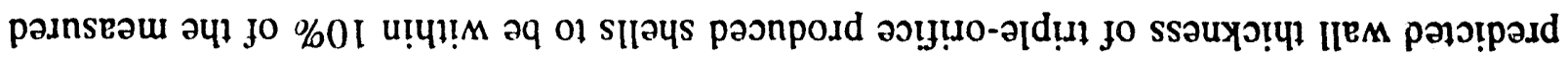




\subsection{REFERENCES}

[4-1] Hansen, C.M., J. Paint Technology, 39, (1967) 104. See also "Handbook of Solubility Parameters and other Cohesion Parameters," CRC Press, Boca Raton, FL 33431.

[4-2] Teas, J.P., J. Paint Technology, 40, (1968) 19. 
SECTION 5

NRL TARGET DEVELOPMENT 


\section{NRL TARGET DEVELOPMENT}

The Naval Research Laboratory (NRL) has been commissioned to build the NIKE laser. In FY93, the task of developing NIKE targets was added to our contract.

\subsection{NRL TARGET FABRICATION GOALS}

Our efforts in FY93 have been directed to five major goals:

1. Production of high quality $\mathrm{CH}$ films.

2. Design and fabrication of mounts for NRL CH-film targets.

3. Development of characterization techniques for $\mathrm{CH}$ films.

4. Assembly of prototype NIKE CH-film targets.

5. Design and implementation of apparatus for cryogenic layer targets.

\subsection{CH-FILMS}

NIKE target specifications call for $\mathrm{CH}$ films whose surfaces (front and back) must be smooth to $10 *$ a averaged over a $2 \mu \mathrm{m}$ square. The specifications also call for a $1.2 \mathrm{~mm}$ square target area. However, a larger area will be advantageous and we have mutually agreed (NRL and WJSA/GA) that the target area will be $2.0 \mathrm{~mm}$ square. The mass densities of the targets are to be in the range of 4 to $8 \mathrm{mg} / \mathrm{cm}^{2}$ which, for the polymers we are currently using (density of about $1.1 \mathrm{~g} / \mathrm{cc}$ ), translates to a thickness range of 35 to $75 \mu \mathrm{m}$. Variations in the mass integral through the film are to be less than or equal to an equivalent thickness variation of $10 \mathrm{~nm}$ averaged over any $2 \mu \mathrm{m}$ square area on the surface of the target film. Any voids in the films should have a scale length less than $1 \mu \mathrm{m}$. These specifications have provided us with interesting challenges in developing techniques by which the high quality films can be produced in the necessary quantities for the NIKE targets needed (250-500 per year). 


\subsubsection{CH-FILM PRODUCTION}

At the beginning of FY93, we selected three methods for producing thin, uniform films of $\mathrm{CH}$ polymer for study and evaluation. These were: parylene coating of flat glass substrates, coating of a glass substrate pulled from a solution of a polymer at a uniform rate, and polystyrene film casting from a solution on a still water surface.

Parylene Coatings. We produced thin $\mathrm{CH}$-films by using a parylene coater. The film quality produced in the coater was better than the $10 \mathrm{~nm}$ roughness maximum specified for the NRL CH-film targets. Further work on the parylene system would be required to demonstrate the capability of producing high quality films in the 35 to $75 \mu \mathrm{m}$ thicknesses required for the NRL NIKE targets. See Section 2.2.2 for a description of the parylene system and its demonstrated capabilities.

Pulled Films. A system was assembled by which glass microscope slides can be pulled at a uniform rate from a solution of polystyrene in suitable solvents. This technique has been used in the past to produce very uniform films of various polymers but with thicknesses in the range of tens of nanometers.

A precision hydraulic cylinder was mounted on a rigid heavy structure to hold, without vibration, at a preset angle, a clean glass microscope slide. The slide was held as shown in Fig. 5-1 so that a film of polymer solution would remain on the flat face of the slide as it was drawn from the beaker. Both the rate of withdrawal from the beaker and the angle at which the slide was held were adjustable. The hydraulic cylinder operation was controlled by gas flow through a micro-control needle valve. The gas pressure was controlled by a regulator attached to a compressed nitrogen gas storage cylinder. It was critical to the operation of the system that the gas flow to the cylinder and the cylinder operation be well controlled, smooth and vibration free.

We produced films of polystyrene on glass slides to demonstrate the operation of the system. The initial films were not high enough quality to be used as target material but did show some promise. However, because of the success of the cast film and parylene film deposition methods, the pulled film technique was not pursued beyond the demonstration phase. The pulled film technique may be useful in the future to produce very thin films for diagnostic use or for other purposes. 


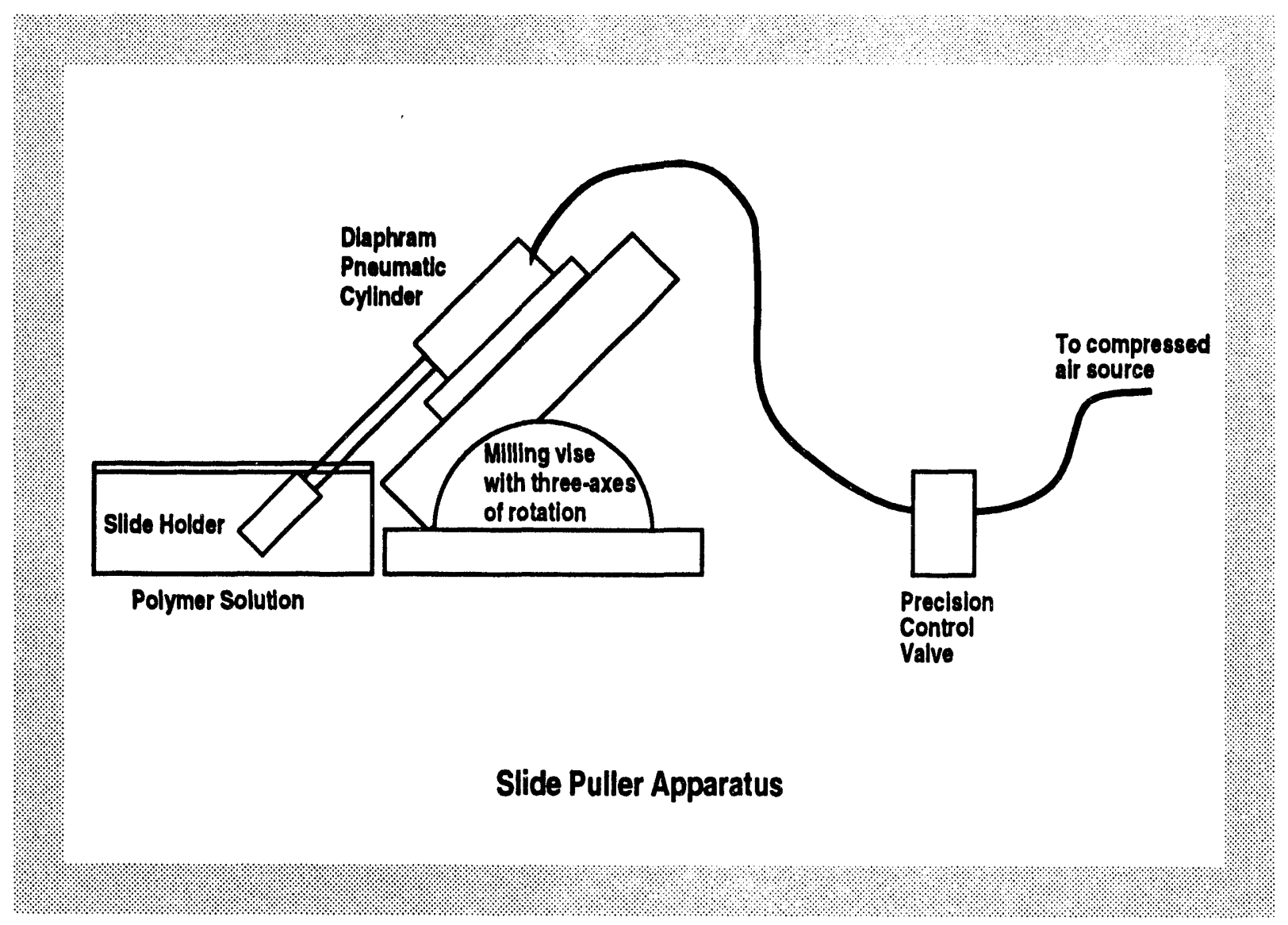

Fig. 5-1. Slide holding fixture for producing thin $\mathrm{CH}$-films.

Cast Polystyrene Films. Polystyrene was chosen as a candidate polymer due to its optical clarity, good manufacturing characteristics, and carbon-hydrogen ratio (1:1). Films of polystyrene had been used previously by the ICF community, but the tolerances required did not approach those required for the NIKE laser experiments. Typically the films had been cast by placing the polymer between two glass optical flats, heating, slowly cooling, and separating. We initially used this technique to produce very flat films, but the surface finish was not sufficient for our needs. Dust, other undesirable particles, and poor wetting by the polymer degraded the surface. As we improved our cleanliness and enhanced the polymer flow, the properties improved, but it became exceedingly difficult to separate the film from the optically flat substrate. We did repeated immersions in hot water followed by freezing. We also tried a release agent, but far too often the film would catastrophically craze before it released from the substrate. 
While we were pursuing the optical flat technique, we were also investigating a float film technique. We dissolved polystyrene in low density solvents, floated the solution on ultra-pure water, and allowed the solvents to evaporate. The first serious problem we encountered was, again, particles, i.e., dust. The particles caused thickened areas of film somewhat larger in lateral extent than the particle itself. The use of a cleanroom aided in the production of films with surface finishes better than required by excluding ambient dust. The initial films made in the clean room were still very thick, $200 \mu \mathrm{m}$ or more. The surface tension differences between the solvents and the water encouraged the solution to puddle. We were able to defeat this phenomenon by adding MEK to the solvents and by altering the casting technique. We now wet the sides of the container with solvent as we apply the solution. This encourages the solution to adhere to the sides, thus forcing it to cover the entire surface of the water. Film thickness is now a function of the weight of solution floated and the percentage of polystyrene in the solution. We have recently produced films as thin as $20 \mu \mathrm{m}$.

We have also overcome non-uniformity flaws in the float films. The flaws had two sources. First, the meniscus at the sides of the container produced a localized thickening at the three point interface. Second, curvature resulted from the water evaporating from beneath the film. (As the water level dropped, the film would bow downward.) The meniscus effect could not be easily eliminated, but it could be made unimportant. By increasing the diameter of the castings, the thickening was totally removed from the area of interest. Our current castings are fifteen centimeters in diameter. The curvature was eliminated by casting the films in a high humidity environment. This reduced the water evaporation without affecting the solvent diffusion. Thus, the films dry before than can bow.

With the production of usable films, we began looking toward production of targets. Polystyrene is known for brittle behavior and crazing. We needed a film strong enough not to break or craze when it is handled, yet not so tough that it would not cut cleanly. The molecular weight of the polystyrene had to be optimized. We studied molecular weights from 50,000 to 200,000 . We have found the higher molecular weights to be the most manageable to date, and will explore even higher molecular weights in the coming year.

To provide adequate records of the parameters associated with every target which is produced, we have instituted a series of procedures designed to make film and target production a fully documented process. We have also initiated a quality control protocol for 
film casting. As soon as a polymer solution is made, a traveler is begun. The solution and its traveler are taken into the cleanroom, and films are cast. The identification number of the casting is based upon the date of casting. After the films are dried, they are given an overall estimation of thickness and uniformity. With our recent acquisition of a $2.5 \mathrm{X}$ interference objective, we have a viewing area $7.8 \mathrm{~mm}$ in diameter. A photograph is taken showing the uniformity over at least a $3 \times 4 \mathrm{~mm}$ area. The photograph is stored in a documentation notebook and the film is stored in a cleanroom container. The characterization data is entered onto the traveler, the container, and entered into a computerized data base. From this database, castings can be chosen as possible target candidates. The casting number later becomes part of the target identification number, thus assuring complete traceability.

\subsubsection{CH-FILM TARGET MOUNT DESIGN AND PRODUCTION}

In a strongly interactive effort with NRL staff members, we designed a CH-film target mount which will satisfy the many requirements associated with the NIKE laser system. A schematic drawing of the resulting target mount is shown in Fig. 5-2. Fig. 5-3 is a photograph of a completely assembled target. Once the design for the mount was completed, drawings were made and several injection molding companies were asked to bid on the fabrication of the necessary molds and production of the mounts. The mounts were made from a polycarbonate material by injection molding techniques and 500 mounts were made for a cost of $\$ 6600$. Using the existing molds, subsequent mounts can be produced for a setup charge of $\$ 450$ plus $\$ 0.30$ per mount. Dimensional tolerances of about 0.001 inch were maintained in the injection molding process. A critical geometrical aspect of the mount is the parallelism of the two edges (not the faces) which determine the "twist" in the film when it is attached to the mount. The two edges must be co-planar so the film is not twisted when it is attached to the mount. We have measured a typical mount and determined that the edges of the attachment faces are co-planar to within 5 milli-radian. The target film mounted between the two faces terminated by these two edges will be flat well within the NRL target specifications.

Mounting techniques and practices have been developed and also learned from other target assembly groups, notably the group at LLNL, so that we are now assembling single film targets which meet the NRL specifications for these relatively uncomplicated targets. In anticipation of the NIKE laser program needs during the next year, we are assembling an 


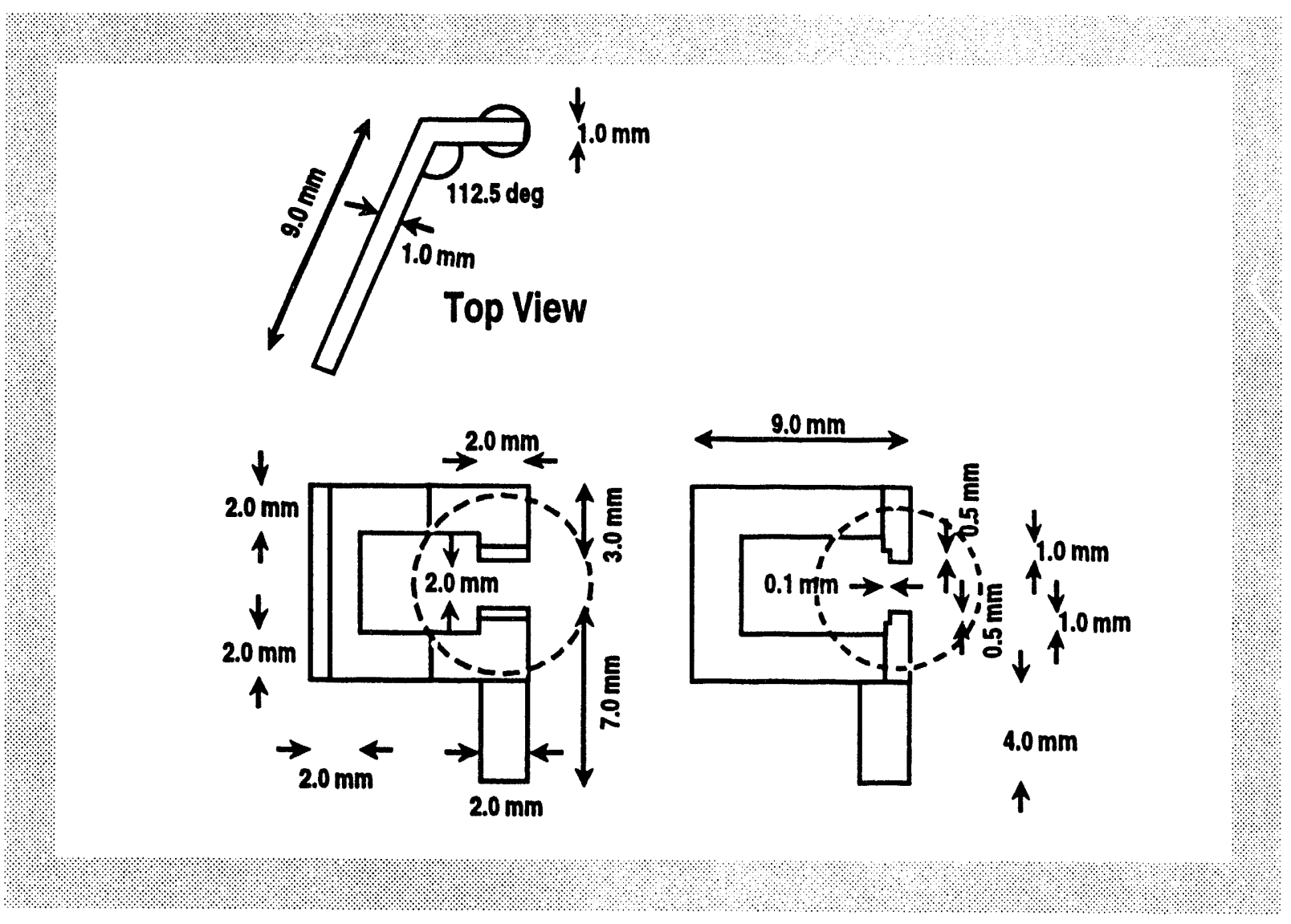

Fig. 5-2. Schematic drawing of the CH-film target mount.

inventory of suitable targets for the program complete with associated paperwork "travelers" listing dimensions, characterization data, and other physical and chemical characteristics of importance to the NIKE program team.

\subsubsection{CHARACTERIZATION OF CH-FILMS}

Optical microscopy, interferometry, and photon tunneling microscopy are the primary techniques used for characterization of the polymer films for NIKE targets. Optical microscopy is used for general physical characterization of film surfaces and overall characteristics and for general dimensional measurements of the films. Interferometry allows us to determine any variations in film thickness and film volume irregularities which transform into equivalent thickness variations or index of refraction irregularities. The Photon Tunneling Microscope (PTM) allows us to quantitatively measure surface irregularities as small as a few nanometers. 


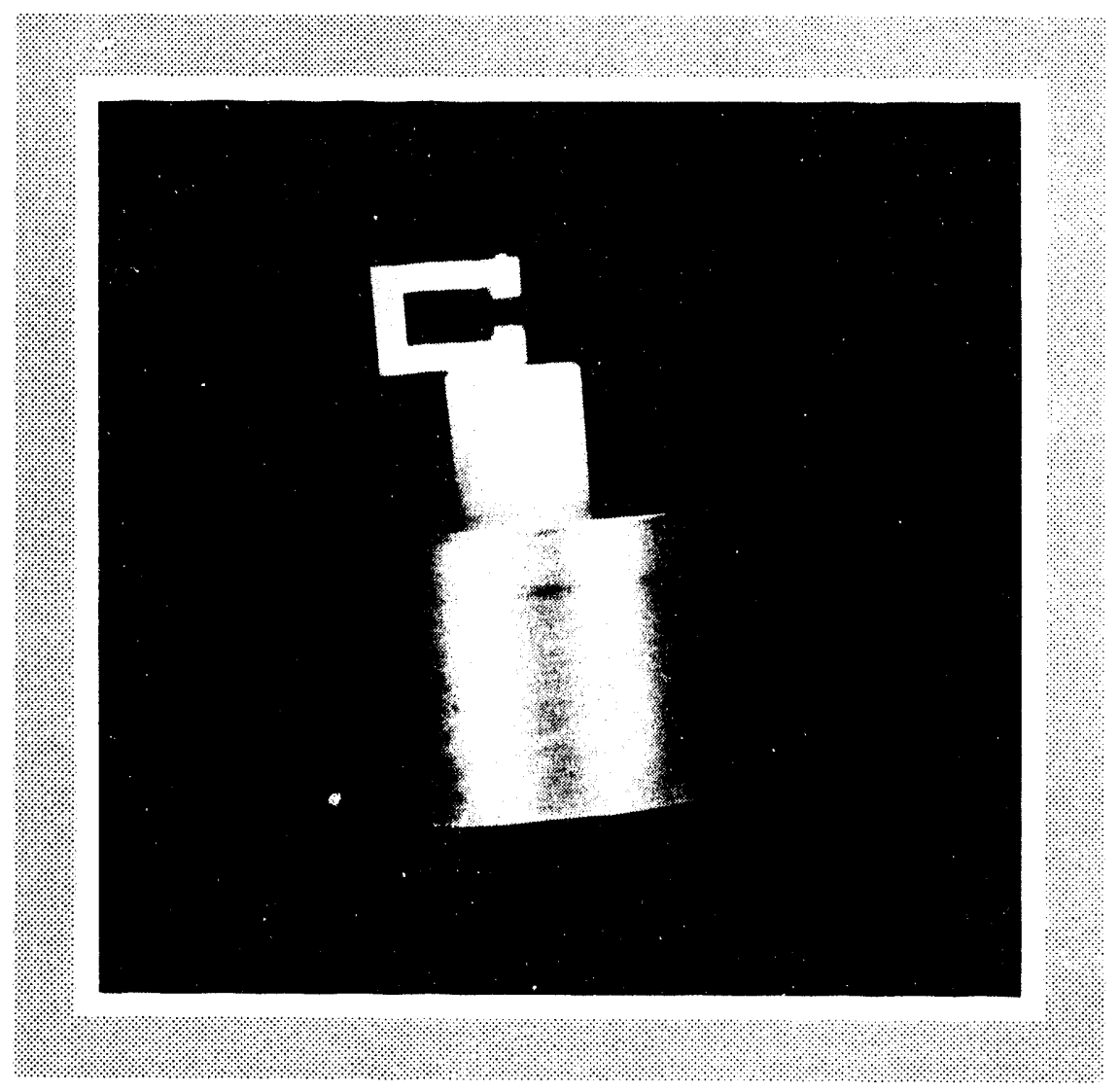

Fig. 5-3. Photograph of an assembled CH-film target.

The optical microscopes we use are a standard Nikon OptiPhot-2 with an additional interferometer attachment and a Unitron with an attached $x-y$ micrometer stage having digital readouts with least significant digits of $1 \mu \mathrm{m}$.

The Nikon microscope and a Zeiss Twyman-Green interference microscope are our primary instruments for high resolution characterization of film thickness and volume regularity. As well as being characterization instruments, these microscopes are invaluable to us in process development efforts providing information on intermediate steps of film casting and various other laboratory tasks.

Optical interferometry provides us with quantitative data on any thickness variations which may be present in the films. For a double pass interferometer such as the TwymanGreen using monochromatic light, thickness variations of 0.1 wavelength are easily to detect and measure by eye and smaller irregularities can be seen by eye and quantified by using more sophisticated data reduction and analysis techniques. 
Interference microscopy also provides a means to observe and measure volume effects if they are present in the films. For example, if we should have a film whose thickness is constant over the entire film but has compositional variations within the film, we can detect and measure these variations insofar as they change the optical properties of the film. Density variations equivalent to thickness variations of $10 \mathrm{~nm}$ will be measurable in the target films. Figure 5-4 is a photograph of an interference pattern of a film which has a thickness variation of less than $10 \mathrm{~nm}$ over a $2 \mathrm{~mm}$ region in the center of the film. The characteristics of the film are well within the specifications for the NIKE targets, i.e., that the film be uniform to $10 \mathrm{~nm}$ averaged over a $2 \mu \mathrm{m}$ square area. For our films, the variation in thickness and surface smoothness can be seen to be less than $10 \mathrm{~nm}$ over a surface more than $1 \mathrm{~mm}$ square.

The newest characterization tool we have is the Photon Tunneling Microscope (PTM) which is proving to be an excellent tool for surface characterization. The operation of this instrument is based on the "frustration" of total internal reflection by a sample surface. The

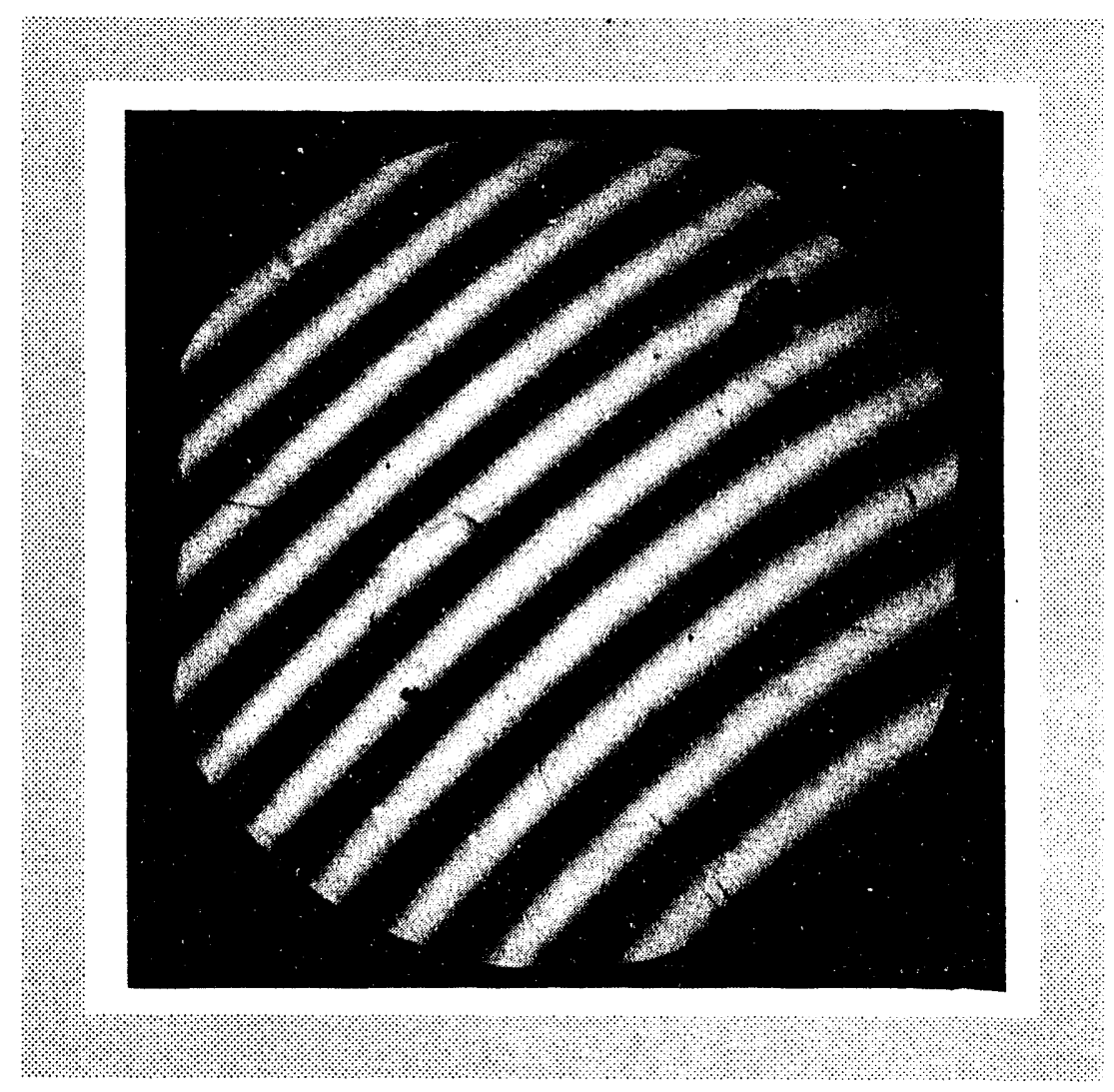

Fig. 5-4. Photograph of an interference pattern of a film which has a thickness variation of less than $10 \mathrm{~nm}$ over a $2 \mathrm{~mm}$ region in the center of the film. 
PTM permits us to quantitatively measure surface defects with a resolution of about $1 \mathrm{~nm}$. Figure 5-5 shows an example of surface morphology obtained from the PTM. The phenomenon of photon tunneling has been known since the time of Newton when he observed the frustration of total internal reflection at the $45^{\circ}$ face of a right angle prism by the near approach of a convex lens surface to the totally reflecting face of the prism. More recently, a photon tunneling microscope was developed and used by John Guerra at Polaroid Corporation in Cambridge, Massachusetts. The PTM system we are currently using is marketed commercially as the Pegasus 1010, by Dyer Energy Systems, Inc., 141 Middlesex Road, Tyngsboro, Mass. With this instrument, we are able to examine a 93 x 119 $\mu \mathrm{m}$ region of a surface with a height resolution of about $1 \mathrm{~nm}$ and a lateral resolution of

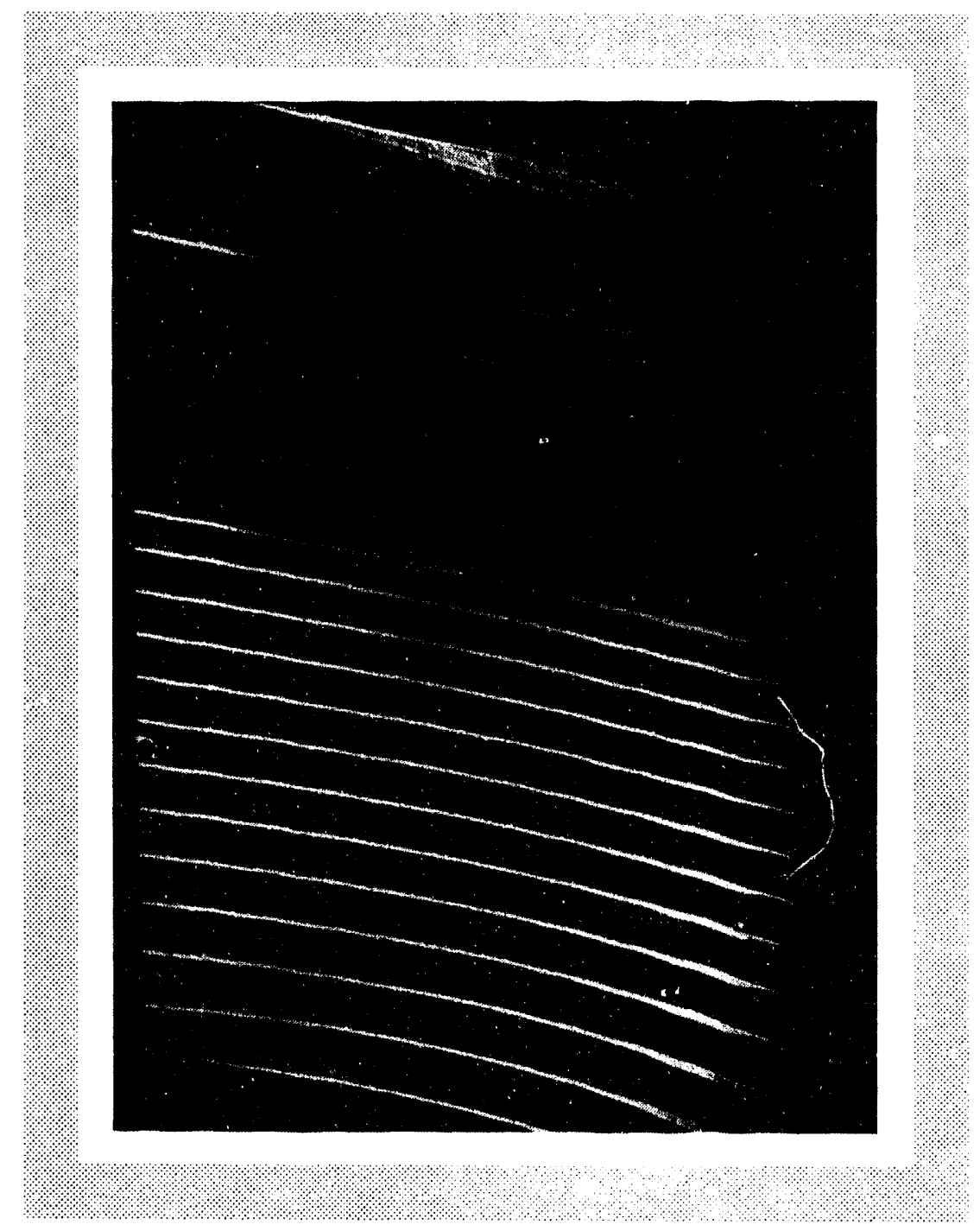

Fig. 5-5. Example of surface morphology obtained from the PTM. 
about $0.18 \mu \mathrm{m}$ when a red filter (600 $\mathrm{nm}$ wavelength) is used in the illumination path. Figure 5-6 shows a $\mathrm{CH}$ film surface on which a scratch about $6 \mathrm{~nm}$ deep has been deliberately incised. Figure 5-7 shows a PTM image of a commercially available Surface Roughness Standard (SRS) in which a series of grooves have been etched in a silicon chip. We use the surface roughness standards for calibration of the PTM. For example, the grooves in the silicon surface of the SRS are $18 \mathrm{~nm}$ deep and $6 \mu \mathrm{m}$ pitch and are virtually rectangular, having flat bottoms and vertical sides. The surface of the silicon wafer in which the grooves have been etched is flat and, therefore, the profile of the roughness standard is a square wave with an amplitude of $9.0 \mathrm{~nm}$ (18.0 nm peak-to-peak) and a choice of several pitches from 6 to $200 \mu \mathrm{m}$.

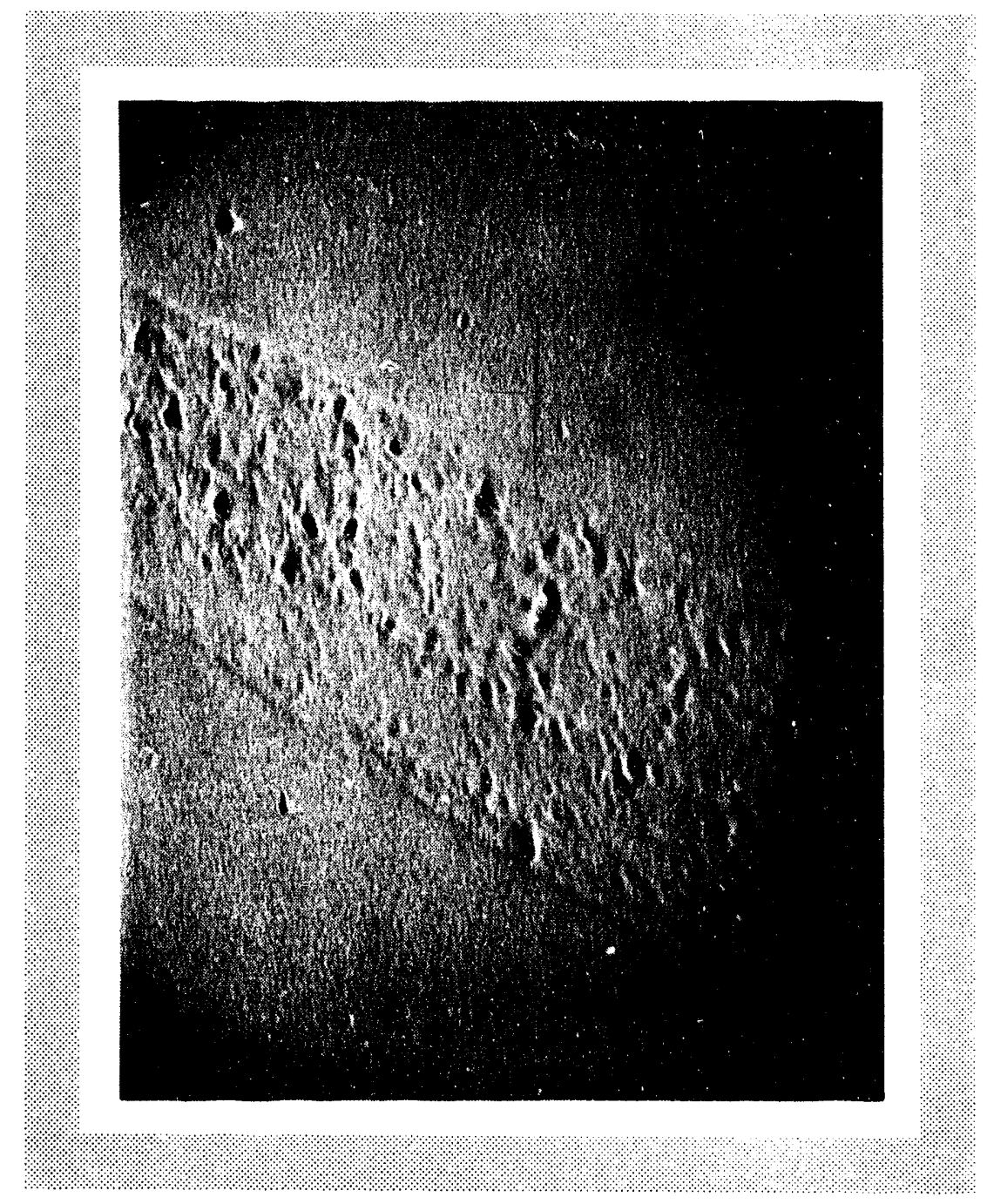

Fig. 5-6. A $6 \mathrm{~nm}$ deep scratch on the surface of a $\mathrm{CH}-$ film surface. 


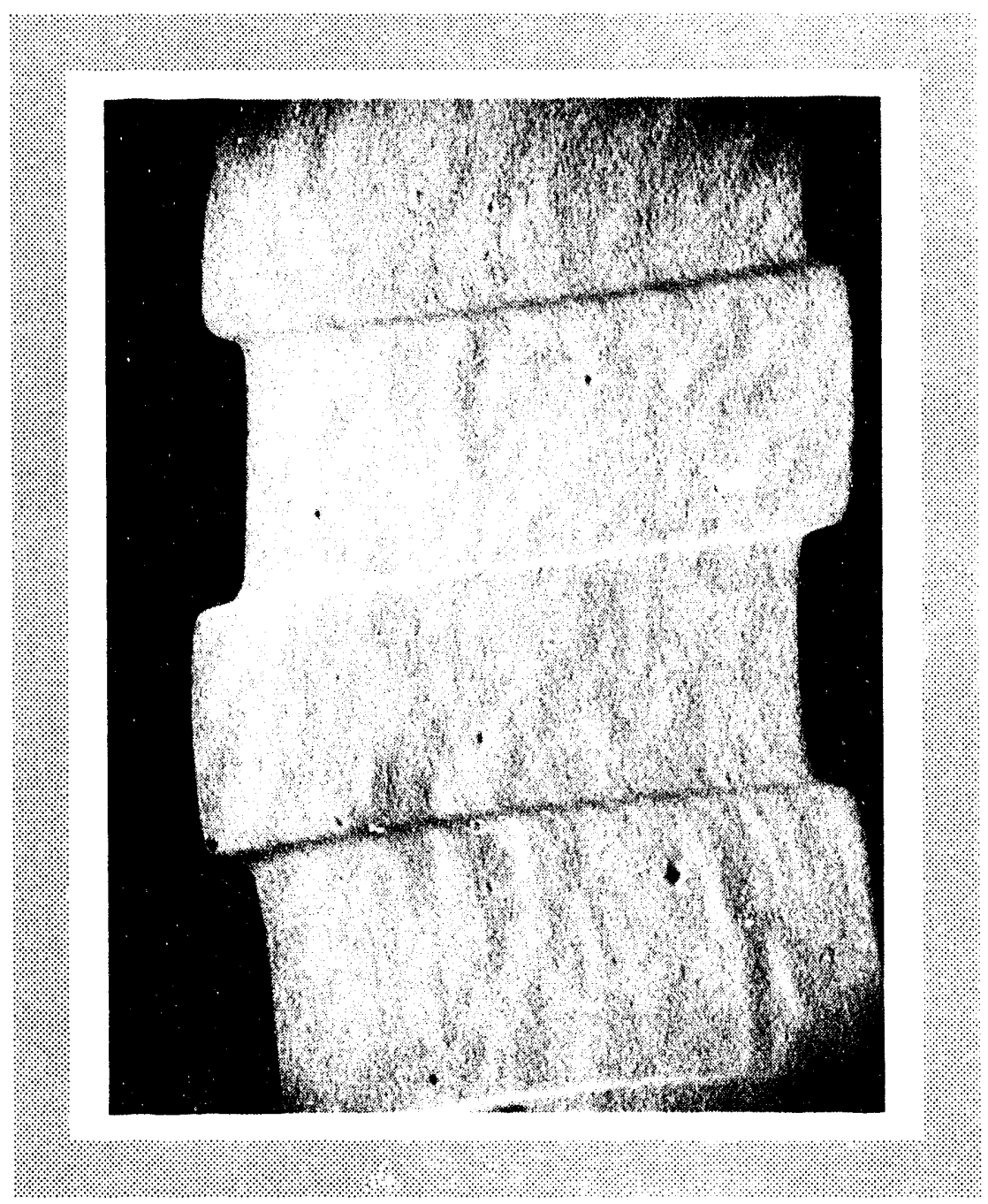

Fig. 5-7. PTM image of sillicon chip roughness standard in which a series of grooves have been otched.

\subsubsection{ASSEMBLY OF PROTOTYPE NIKE CH-FILM TARGETS}

Prototype CH-film targets were assembled and delivered to NRL for evaluation by the NIKE program staff. $\mathrm{CH}$-film targets are being assembled on a routine basis and stored to be used as needed by the NIKE program.

Assembly of the NIKE targets with the accuracies specified by NRL has presented some interesting and often difficult challenges. The problems of producing the individual precursor 
parts of the targets, e.g., the polystyrene cast films and the target mounts, have been solved to a first approximation. As the targets are used in the NIKE laser program, there will no doubt be requests for modifications which may necessitate changes in the mount configuration. Changes are always expected in a research program and provide us with further interesting challenges.

The sections of polystyrene film required for the targets are $2 \times 4 \mathrm{~mm}$ rectangular pieces cut from the $15 \mathrm{~cm}$ diameter circular film disks, fabricated and characterized as discussed in previous sections. The edges of the mounting faces on the target mount are spaced $2 \mathrm{~mm}$ apart so that a clear $2 \times 2 \mathrm{~mm}$ area is accessible to the primary NIKE laser beam (see Fig. 5-2 for target mount dimensions and configuration). The additional $1 \times 2 \mathrm{~mm}$ areas at the ends of the film section are sufficient attachment sections to hold the film on the mount. The sections are cut from the large circles of film by using a heated $25 \mu \mathrm{m}$ diameter tungsten wire as a knife. The large film and the hot wire are mounted on micromanipulators which allow an operator to rapidly cut several film sections in a rectangular grid pattern from the large films. The hot wire arrangement and the cutting technique are illustrated in Fig. 5-8. The wire is maintained at a temperature high enough to cut the film without cracking but low enough that melting and

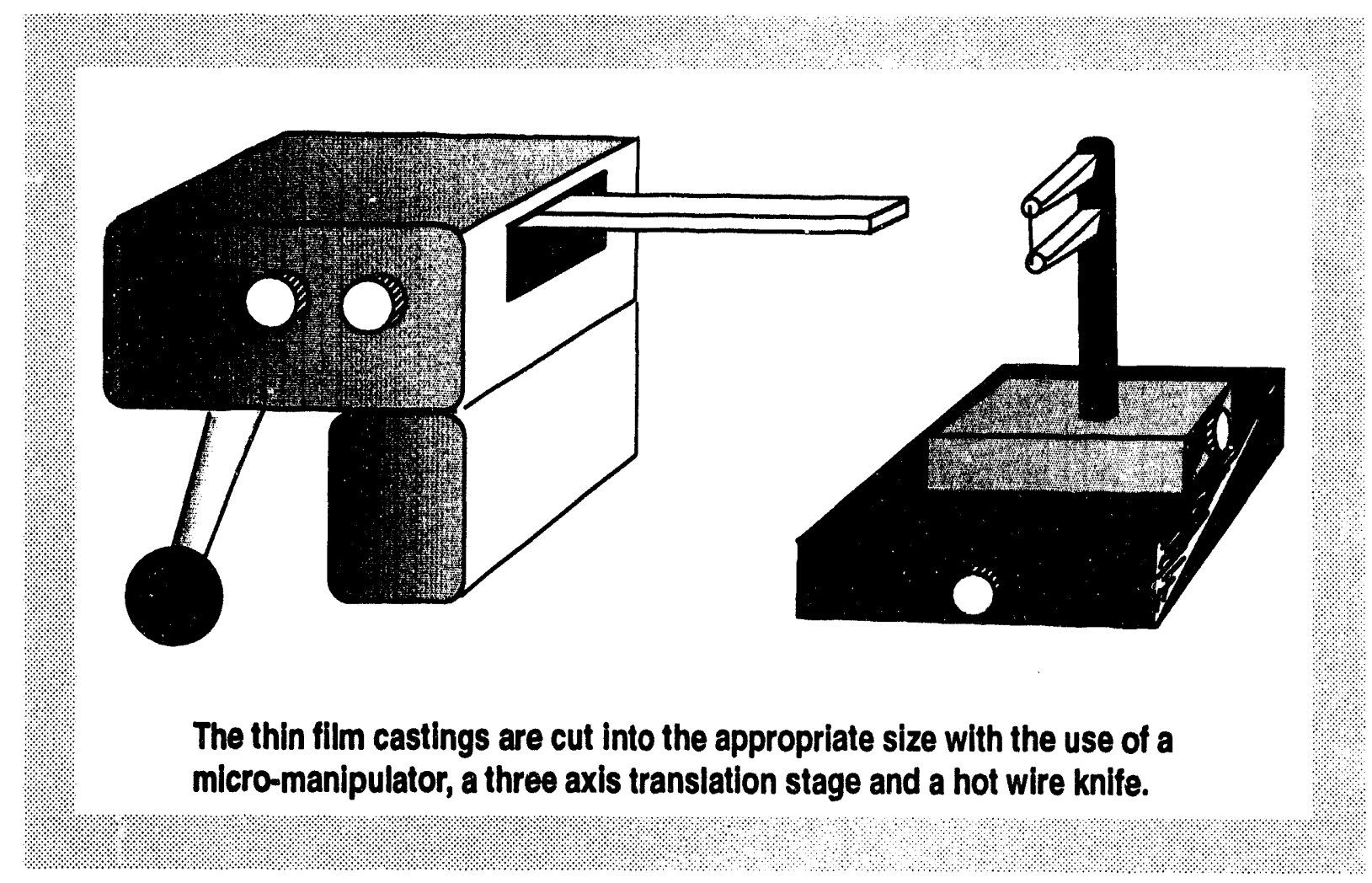

Fig. 5-8. Hot wire arrangement for cutting $\mathrm{CH}$-films. 
beading of the film along the cut edges does not occur. The cut sections remain very loosely attached after the cutting process and can be easily picked up at the non-target end tabs by using a vacuum chuck attached to a micro-manipulator.

The target mount is held in a special fixture and the target film is brought into position by the vacuum chuck. Epoxy (Norland Optical Adhesive, UV-curable) is applied to the appropriate faces of the target mount and the micro-manipulator is used to move the film into contact with the epoxy. The film is held in position and an intense ultraviolet light is focused on the epoxy to cause it to cure. The mounted target film is re-characterized to determine that the film has not been damaged during the mounting process and meets the flatness specifications.

The completed target is stored in a dust free container and placed in inventory for subsequent transport to NRL. The storage container and a completed target are shown in Fig. 5-9. The polyethylene containers are obtained commercially and a special delrin insert is attached inside the container to hold the target to prevent damage during transport.

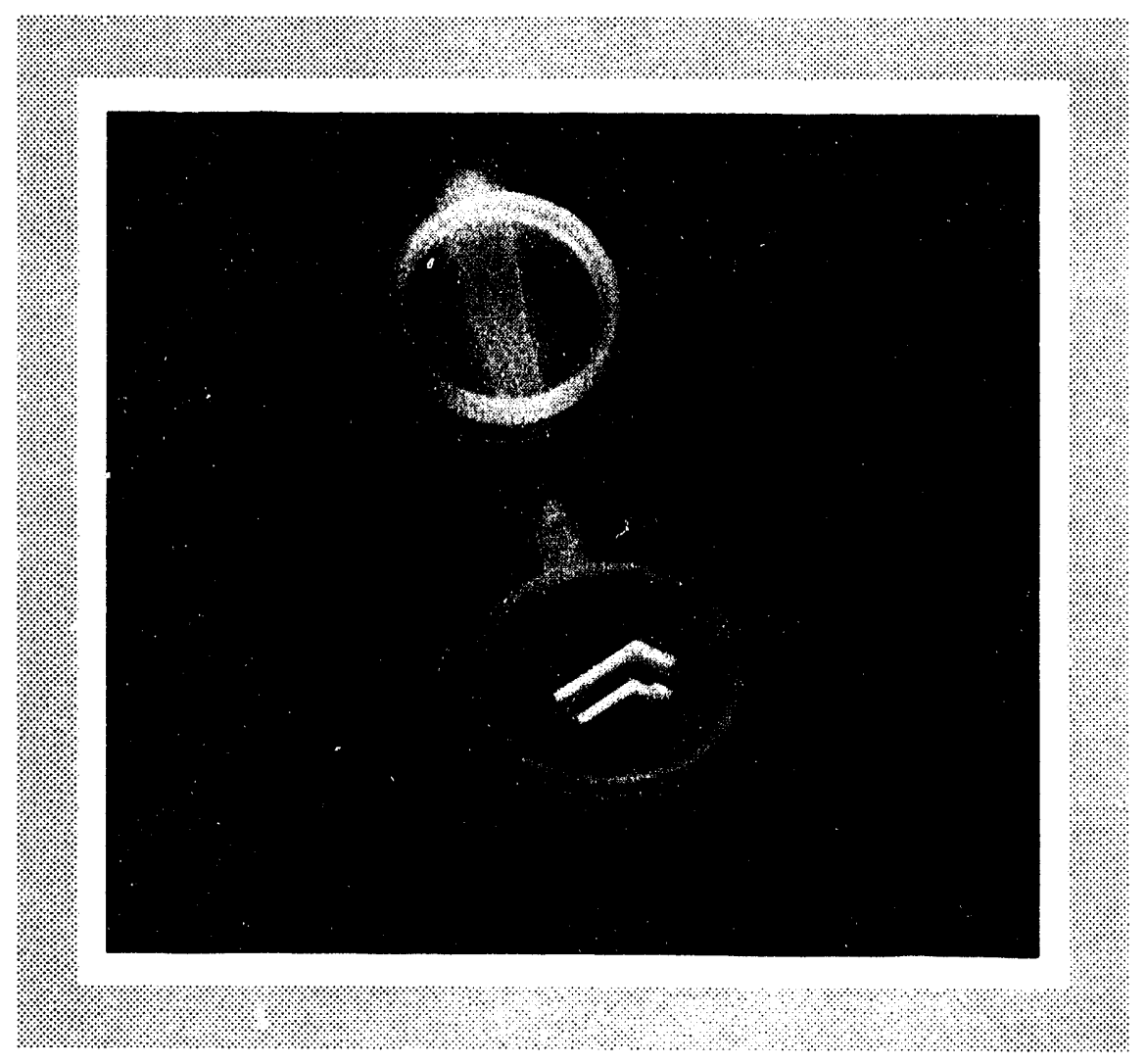

Fig. 5-9. Completed $\mathrm{CH}$-film target in its storage container. 


\subsection{DESIGN AND IMPLEMENTATION OF APPARATUS FOR CRYOGENIC LAYER TARGETS}

We have designed and fabricated much of the apparatus necessary to begin studies on the production of planar layer cryogenic targets for the NIKE laser experiments. Temperature measurement and control systems and a high vacuum pumping station have been purchased and a liquid helium dewar has been ordered. The cryogenic subtask was removed from the NR01 statement of work in FY93, and no cryogenic effort on this task is anticipated during FY94.

For additional information, please contact Dr. C. Hendricks (WJSA). 


\section{FABRICATION OF FILMS AND FILTERS FOR SNL}

An important task in FY93 was the fabrication of flat films and filters for Sandia National Laboratory (SNL).

\subsection{FABRICATION OF FOILS AND FILTERS}

The purpose of this task was to fabricate flat foils and filters supported on various frames for diagnostic measurements in PBFA II and other ICF machines at SNL. The types of foils and filters fabricated were dictated by requests from SNL. Generally, initial deliveries were made soon after the request was received. In the case of orders for 50 to 100 foils, however, completion of a delivery often required several months.

In FY93, SNL requested foils of the following four types:

1. Thin layers of gold on thin Mylar films

2. Free-standing foils of gold

3. Free-standing foils of aluminum

4. Duplex free-standing foils of gold and aluminum

The frames used to support the foils were fabricated to meet specifications supplied by SNL. Examples of the various frames used are shown in Fig. 6-1. They included large titanium oval frames, small brass oval frames, and small circular brass and steel frames.

Fiducials were often required on the foils. For gold-on-Mylar films, these fiducials could be areas where there was no gold coating, but most often were holes in the film. For the freestanding foils, the fiducials were always holes in the foils.

For the gold-on-Mylar films, fabrication of the film was straightforward. The Mylar was cemented to the frame; the frame was placed in a holder; and gold was deposited on the Mylar in the cathodic sputtering device shown in Fig. 6-2. When uncoated regions were specified or 


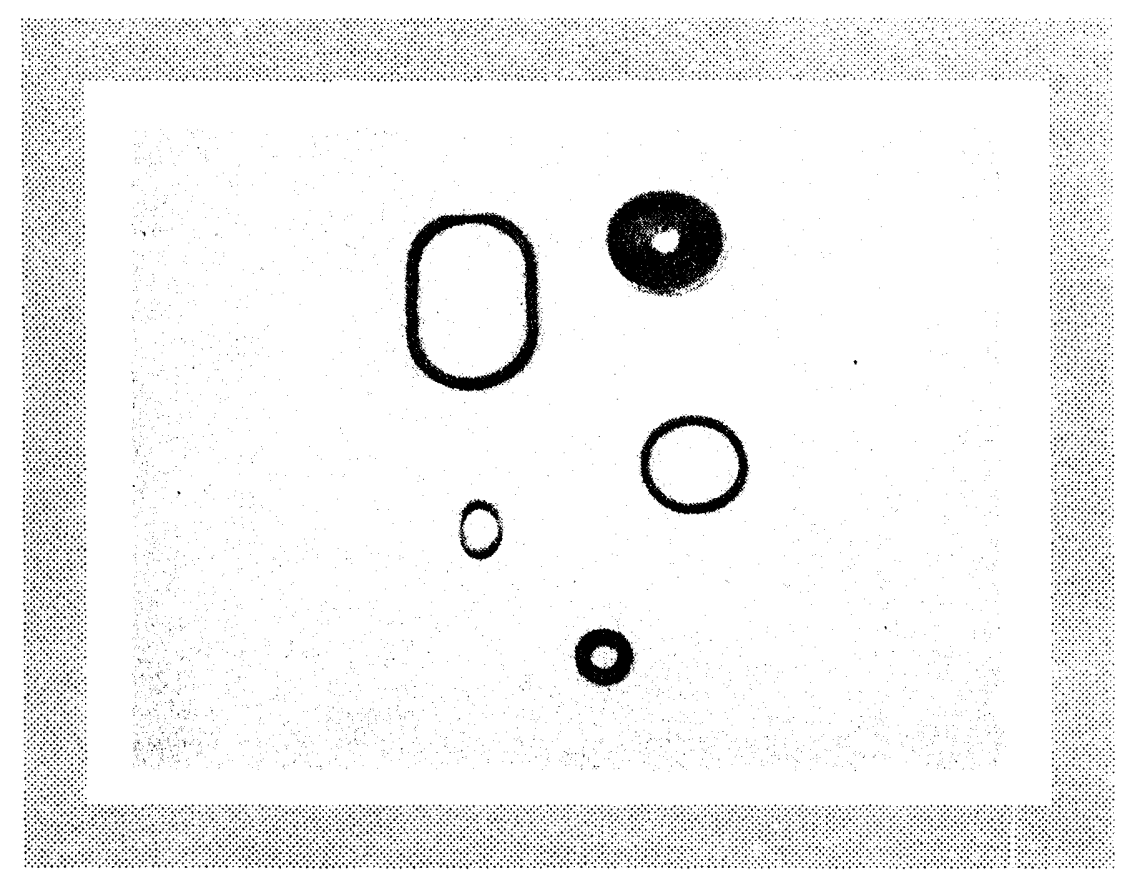

Fig. 6-1. Examples of frames used to support folls requested by SNL.

when different gold thicknesses were desired on different regions, masks were used to shield the regions from gold deposition. Fiducial holes were punched in the films using a special hollow punch fabricated at GA.

Fabrication of the free-standing gold and aluminum foils was somewhat more complex than fabrication of the gold-on-Mylar films. First, a cellulose acetate film, about $1 \mu \mathrm{m}$ thick was cemented to a large ring. Then, either aluminum or gold of the desired thickness was deposited on the cellulose acetate. Next, the specified frame was cemented to the metal, and the frame was cut from the larger ring. If fiducial holes were specified in the foil, they were punched in the foil at this time, while it was still supported by the acetate film. Finally, the cellulose acetate film was dissolved away in methyl acetate leaving the metal film free-standing on the frame.

Foil thicknesses were determined for each film and filter fabricated. This was done using alpha particle spectrometry to measure the energy shift of $5.48 \mathrm{MeV}$ alpha particles on transmission through the foil. Standard expressions were used to calculate the film thickness form the energy shift. Additionally, when fiducial holes were present, we measured their spacing using an optical comparator. 


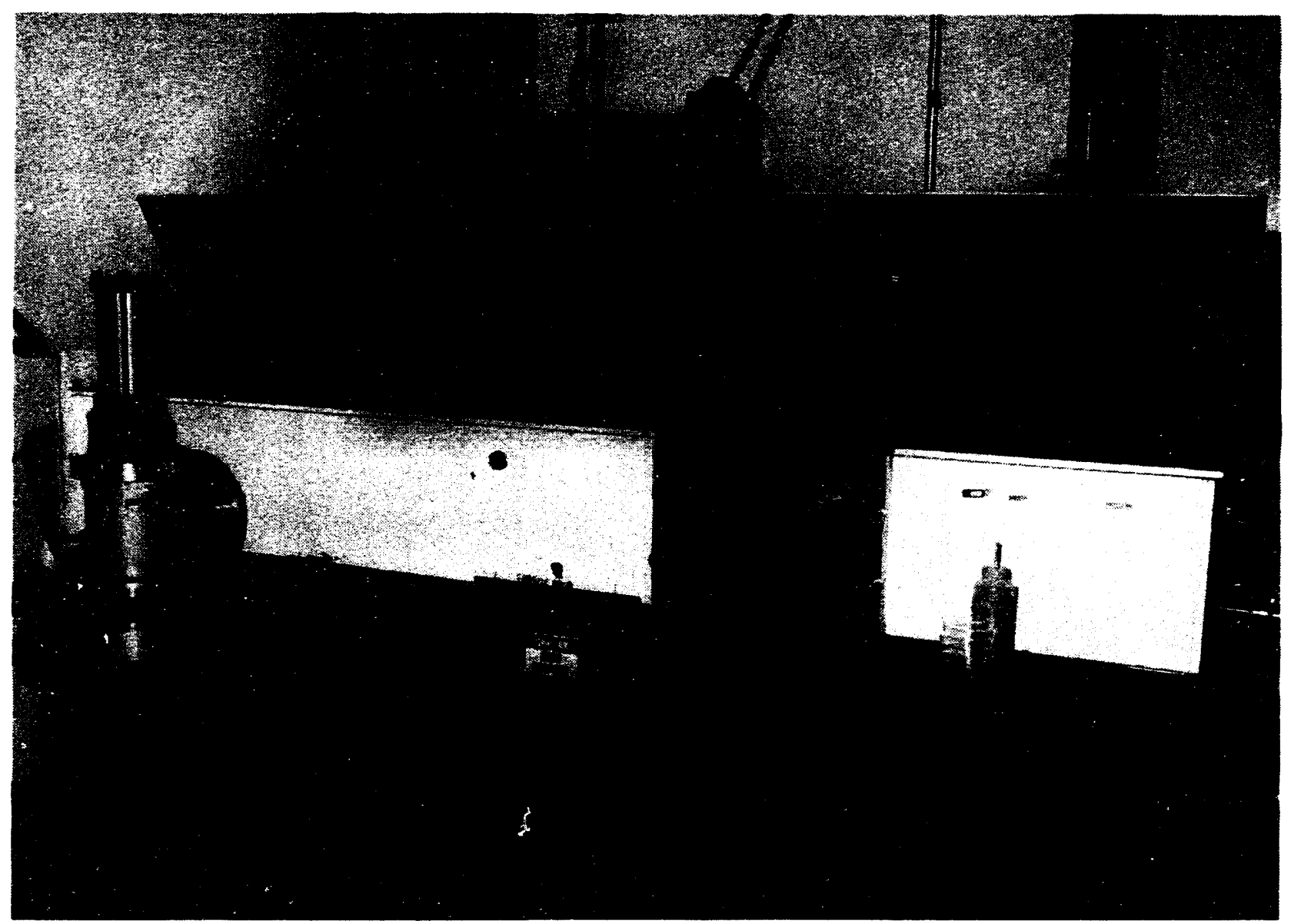

Fig. 6-2. Cathodic sputtering device used to deposit gold on mylar. 
A photograph of the different types of foils and filters fabricated this year is shown in Fig. 6-3. The numbers of the different type of foils and filters fabricated at GA in FY93 are listed in Table 6-1.

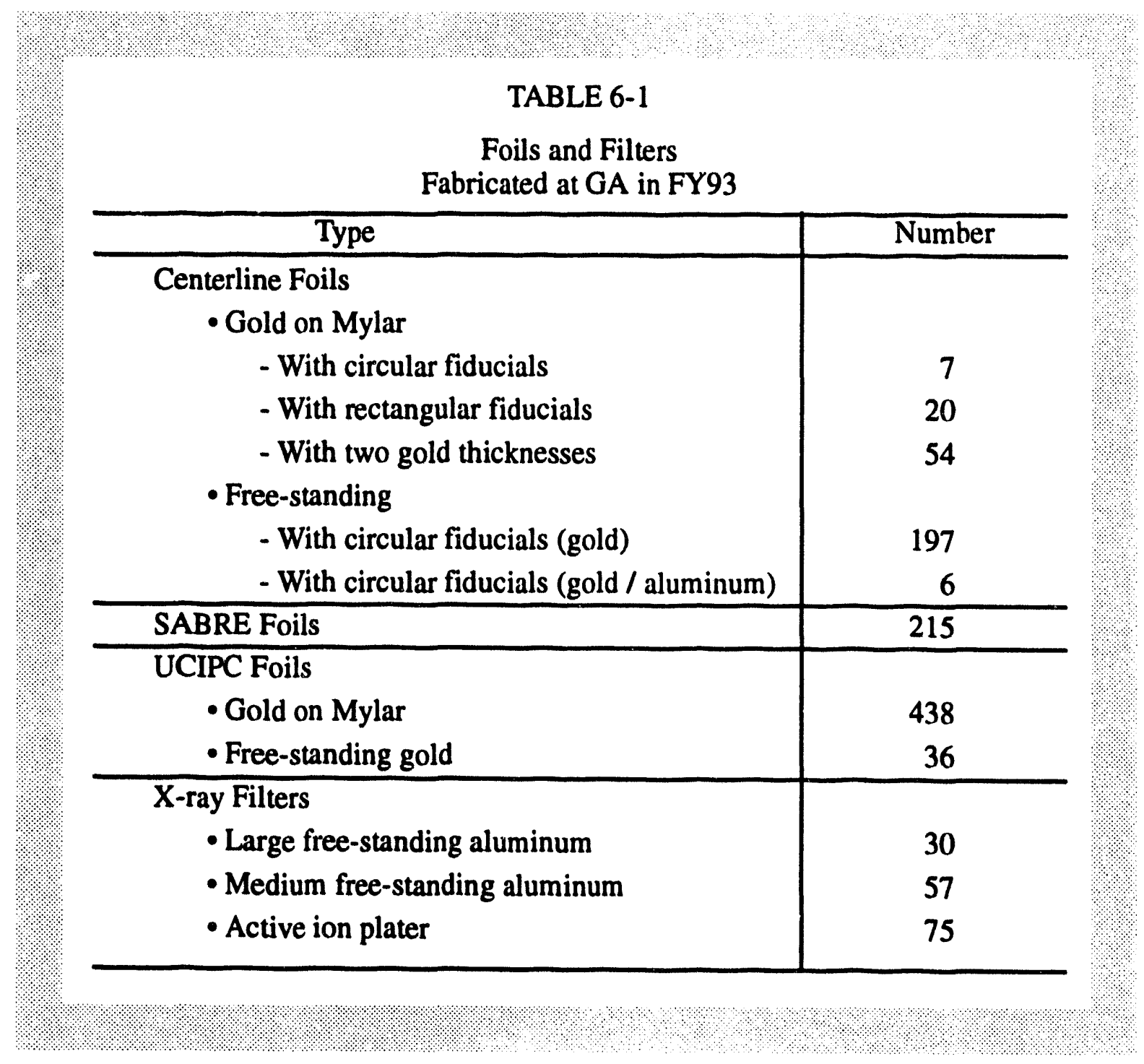

\subsection{SUPPORT TO SNL}

An engineer, Tom Alberts, is assigned to Sandia National Laboratory in Albuquerque, New Mexico. His major tasks are to act as a liaison between GA and SNL, fabricate foils and filters with SNL facilities, and develop xray characterization of filters. 


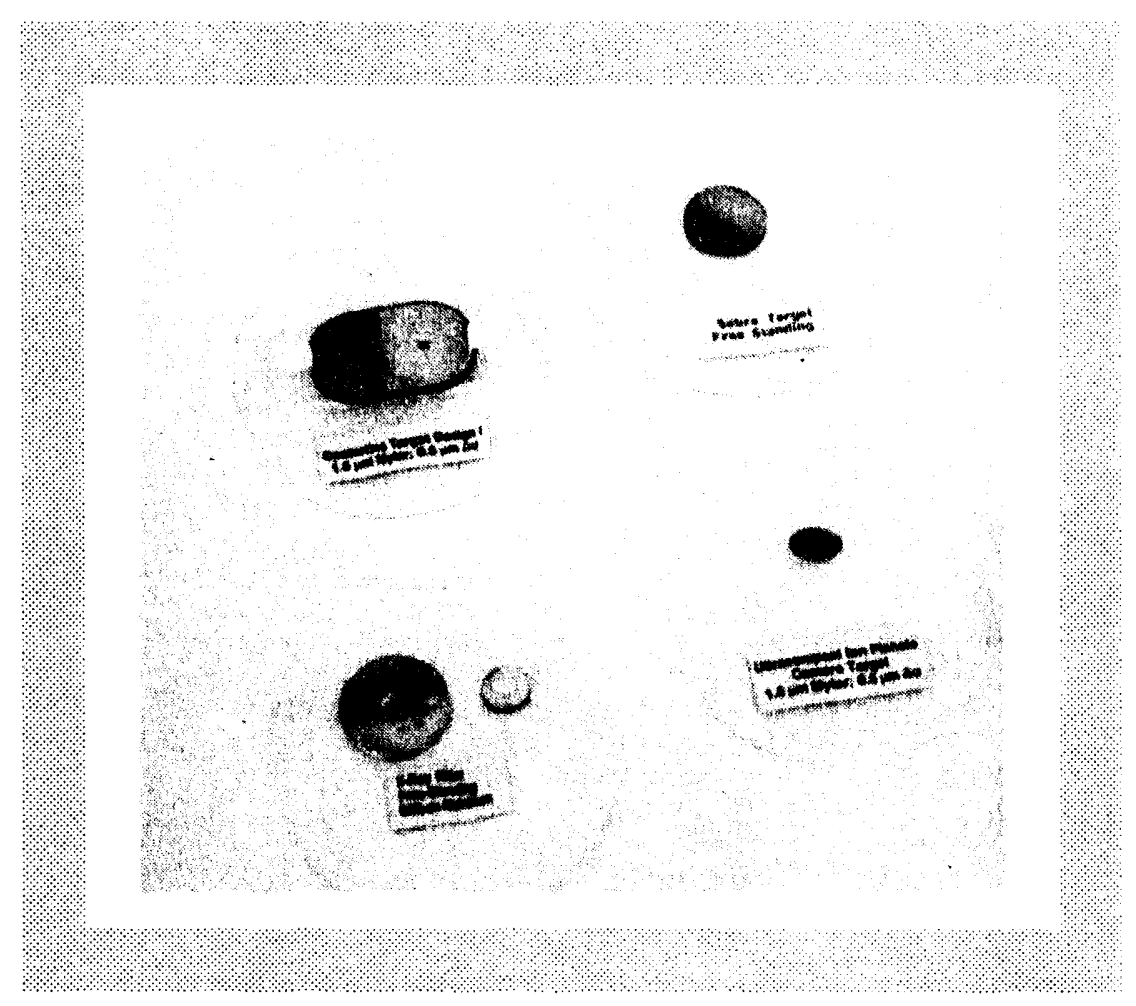

Fig. 6-3. Different types of foils and filters fabricated for SNL in FY93.

As liaison, the engineer accepts deliveries from GA, documents characterization with SNL format, and places them into SNL inventory. He also works with SNL personnel in coordinating and transferring specifications for deliveries.

Various flat foils were fabricated for use as targets and filters in support of PBFA II, SABRE, and Two-Stage facilities. The support at SNL addresses quality of fabrication and characterization of these foils as well as meeting short notice delivery schedules. The following list describes foils fabricated during FY93:

- Three centerline targets were fabricated for PBFA II facility. These foils were a prototype design made of parylene-N/AV/Au.

- 113 targets were fabricated for the SABRE facility. These foils were made of Au. Production of these targets has been transferred to GA.

- $530 \mathrm{x}$-ray filters were fabricated for use during the February 1993 target series. The materials used for fabrication of these filters included: $\mathrm{Al}, \mathrm{Ag}, \mathrm{Au}, \mathrm{Cu}, \mathrm{Fe}, \mathrm{In}, \mathrm{Ni}$, $\mathrm{Si}, \mathrm{Sn}, \mathrm{Ti}, \mathrm{V}$, Mylar, parylene-D, parylene-N, and Saran. 
At SNL free-standing foils are fabricated by vacuum depositing the electron-beam evaporated foil material onto a glass slide that has been coated with a water soluble releasing agent of either $\mathrm{BaCl}$ or detergent. The foil can then be removed when the releasing agent is dissolved in water. Handling of the foil at this stage is critical to prevent tearing of the foil and to control formation of wrinkles.

The effort to develop $x$-ray characterization of filters is ongoing. This task utilizes equipment available at SNL. Presently, a single-anode 5000 Volt Manson $x$-ray source, a Manson gas proportional counter, and a Canberra Multi-Channel Analyzer have been tested and operated. A simple paddle stage operated with a linear vacuum actuator holds filter samples for measurement of $\mathrm{x}$-ray transmission.

For further information, please contact Dr. J. Kaae (GA) or T. Alberts (WJSA). 
SECTION 7

\section{OMEGA UPGRADE CRYOGENIC TARGET}

DELIVERY SYSTEM 


\section{OMEGA UPGRADE CRYOGENIC TARGET DELIVERY SYSTEM}

An important task in FY93 was the development and design of a cryogenic target delivery system for the OMEGA Upgrade facility at the University of Rochester.

\subsection{INTRODUCTION}

The upgrade of the OMEGA laser at UR/LLE to $30 \mathrm{~kJ}$ will result in a need for millimeter-size ICF capsule targets filled with $\mathrm{D}_{2}$ or DT and maintained at cryogenic temperatures. This mandates a cryogenic target delivery system capable of filling, layering, characterizing and delivering cryogenic targets to the OMEGA Upgrade target chamber. GA has been assigned the task of designing the cryogenic target delivery system. The program goal is to $d$ ign, construct, and test the entire target delivery system by 1998 . We have taken a concurrent engineering approach to the design of the target delivery system, breaking it down into modules: Target Support, Cryogenic Fill Station, Transport, Layering, Characterization and Target Insertion. During FY92, we completed both the Design Requirements Report [Ref. 7-1] and the Conceptual Design Report [Ref. 7-2]. During FY93, we were tasked with the following activities:

(A) Layering R\&D to devise and demonstrate a viable way to produce uniform $D_{2}$ and DT fuel layers on a time scale appropriate for making OMEGA Upgrade decisions. Thermal gradient layering was to be evaluated for its suitability for production of OMEGA Upgrade cryogenic targets. As part of the layering subtask, we were requested to develop an R\&D program plan for determining acceptable cryogenic target layering techniques.

(B) Characterization R\&D to devise and demonstrate characterization techniques capable of characterizing millimeter diameter transparent shells with hydrogenic fuel layers up to $100 \mu \mathrm{m}$ thick. A Mach-Zehnder interferometry system was to be constructed and evaluated with respect to the layering techniques described in Subtask (A). 
(C) System engineering to continue engineering activities on target insertion system modules that were not directly impacted by the choice of layering and characterization methods (Subtasks A and B). Engineering activities included: designing and demonstrating proof-of-principle of the high pressure (1500 atmosphere) cryogenic booster system for the LLE DT fill station, design for the method for handling (removal and transport from the DT fill station) of cryogenically cooled DT-filled targets, and a design for cryogenic storage of DT and $D_{2}$ filled targets. We were also requested to develop a program plan for performing the design and construction of the OMEGA Upgrade Cryogenic Target Handling System. This was to include a Work Breakdown Structure (WBS), a project schedule, and a project cost estimate based on the WBS.

\subsection{LAYERING}

As part of the layering task, a layering plan was delivered to the University of Rochester that outlined a strategy for acquiring the information necessary to make a decision on plausible layering techniques for the cryogenic target delivery system. The plan addressed issues that need to be resolved for both solid and liquid layering as well as the development of characterization techniques. The document listed the target requirements relating to layering, defined and ranked the layering concepts, and provided flow diagrams ("decision trees") of the logic involved in performing the progression of experiments. An example of a flow diagram is shown in Fig. 7-1 for liquid layering. This report will soon be superseded by a national cryogenics layering program which incorporates issues that need to be addressed for both OMEGA Upgrade and the National Ignition Facility.

Additional activities included support of the experimental layering activities at LLNL and phenomenological modeling of the thermal gradient layering of $D_{2}$. Millimeter-size polystyrene capsules have been made for use in liquid layering experiments. Some of the $1 \mathrm{~mm}$ capsules had plastic fill tubes chemically welded to them. Others were coated with GDP for strength enhancement for use in a "clamped shell" experiment. We also provided technical support during the assembly of the cryostat which will hold the clamp shell experiment.

A modeling effort developed a simple model to increase our understanding of the physics of the liquid layering process. The calculations were initially performed to compare with the work of V. Varadarajan, et al. [Ref. 7-3], on a small shell and then scaled to a $1 \mathrm{~mm}$ capsule with a $100 \mu \mathrm{m}$ thick $D_{2}$ layer. The model predicts that an impractically 
Is there a credible conceptual design for

layering and characterization of liquid

layers at the center of the chamber?

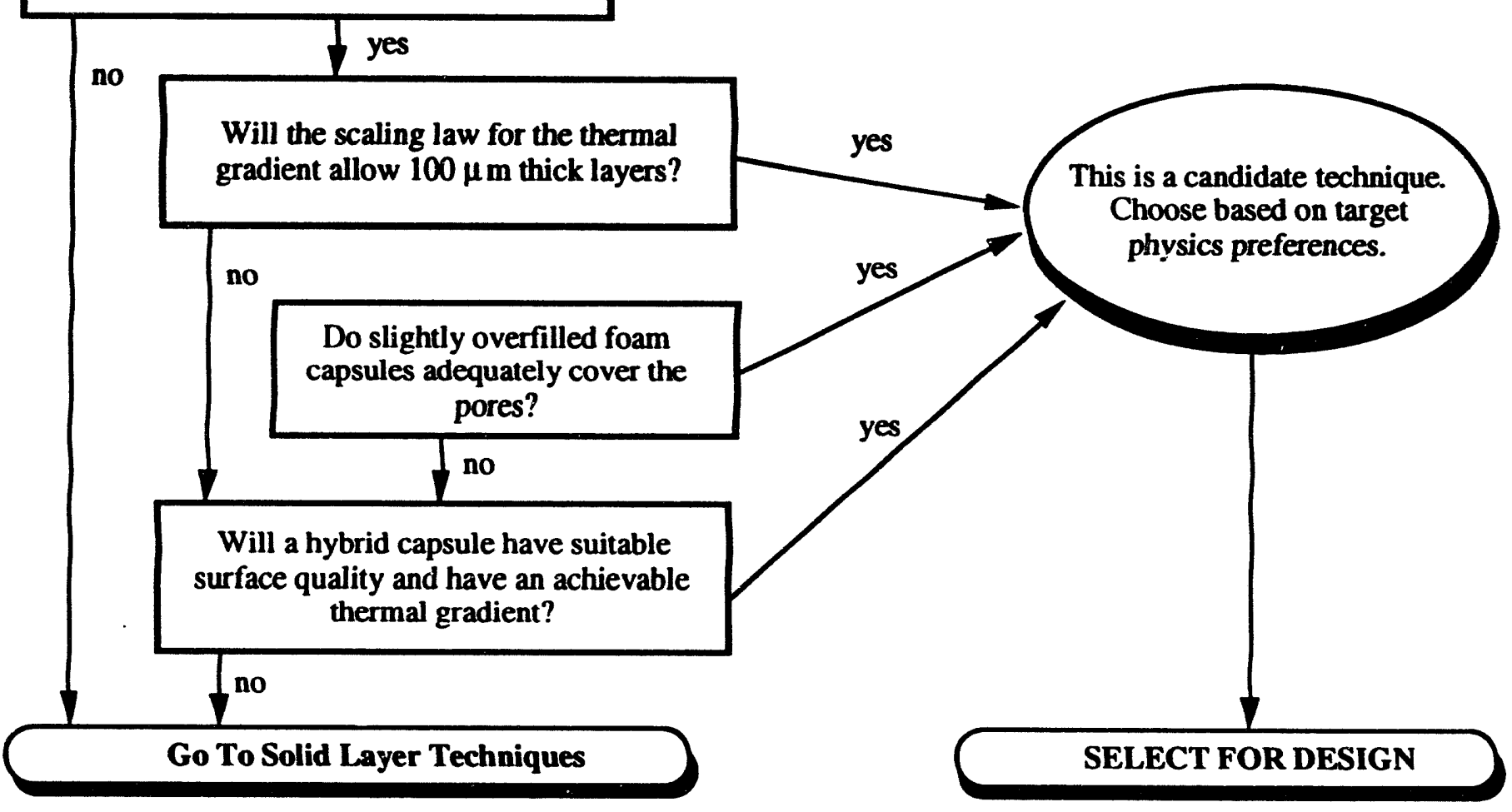

Fig. 7-1. Liquid layering flow diagram ("decision tree"). 
large temperature gradient is required. This implies that assistance is needed to support a $100 \mu \mathrm{m}$ thick liquid layer.

For further information, please contact Dr. D. Bittner (WJSA).

\subsection{CHARACTERIZATION}

Two Mach-Zehnder interferometers were borrowed from the University of Rochester. These two systems were used on the OMEGA tank for in-situ target characterization. Both instruments were set up and tested at WJSA with the help of Mark Wittman of LLE. Subsequently, one of the interferometers was taken to LLNL for use on the experiments in the cryogenics laboratory.

The second interferometer is in the process of being modified under the guidance of the University of Rochester. These modifications are necessitated by the increase in wall thickness associated with the larger capsules which will be used on OMEGA Upgrade experiments. The modification involves changing from a planar reference wavefront to a curved wavefront. In the current configuration of the interferometer, a planar wavefront is interfered with the curved wavefront of the capsule. The fringes in the observed interferogram indicate the differences between the capsule image and the planar wavefront. A perfect capsule will have a "bull's-eye" fringe pattern concentric with the center of the capsule. As the capsule walls become thicker, the fringe density in the interferometer image becomes more dense. Deviations from a perfect "bull's-eye" pattern become difficult to detect. In the current configuration both beam paths contain the capsule image in a planar background and the interference is accomplished by rotating one of the mirrors, shearing the image. By using a curved reference wavefront, the interference occurs between two curved wavefronts. In this configuration, the interferogram shows differences between two curved wavefronts in the region of the capsule. In the background there will be fringes due to the interference between the planar and curved wavefronts. This modification will reduce the fringe density problem one has with a planar wavefront. For example, if the curved reference wavefront is identical to that from the capsule, there will be a constant phase across the capsule image. In our case, the modification was implemented by first removing the shearing mirror, coupling into the incident laser beam with one end of a fiber optic arrangement, and finally aligning the other end of the fiber optic arrangement colinear with the reference mirror's reflected beam path.

For further information, please contact Dr. D. Bittner (WJSA). 


\subsection{SYSTEM ENGINEERING}

Several support systems must be engineered for the cryogenic target delivery system for the OMEGA Upgrade laser. These systems include: target support, fuel layering and characterization, and cryogenic transport and storage.

\subsubsection{TARGET SUPPORT}

The purpose of the target support is to hold a filled target at the focus of the OMEGA Upgrade laser beams and not interfere with any of the sixty laser beams during the laser shot. Additionally, the target support must maintain a mechanical rigidity to better than $\pm 5 \mu \mathrm{m}$ and not cause a major perturbation on the target surface finish as this could initiate RayleighTaylor instabilities. A tripod mounting technique (Fig. 7-2) was selected as the preferred design based on these considerations.

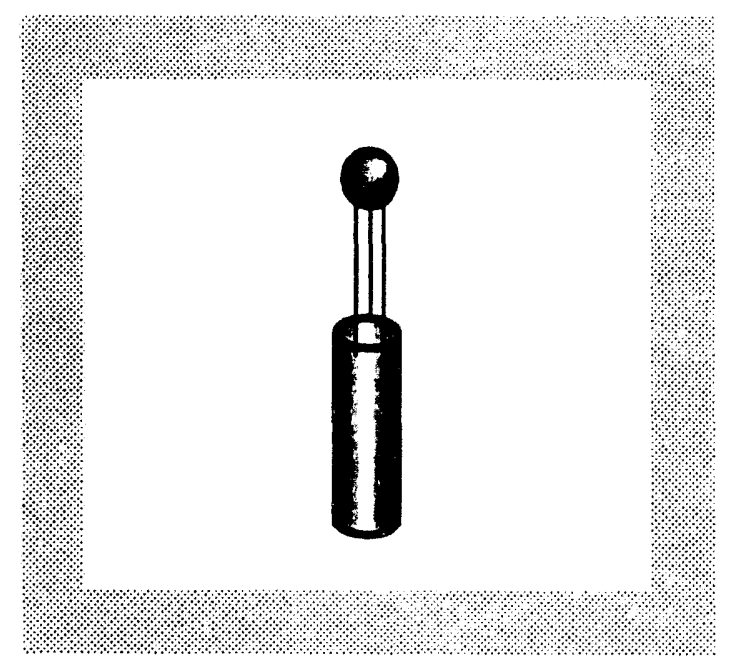

Fig. 7-2. The tripod mounting tochnique for supporting ICF targets.

In this configuration, the target is supported on three P-100 carbon fiber legs that are tapered from $1 \mu \mathrm{m}$ at the tips to $8 \mu \mathrm{m}$ at the base. The legs are parallel to each other, buttmounted to the target (at the tips) on a $300 \mu \mathrm{m}$ diameter. This configuration was analyzed to determine the natural frequencies of the bending modes. Three cases were examined, for carbon fiber lengths of 3,4 , and $5 \mathrm{~mm}$. The finite element code COSMOS was used to determine the natural frequencies. The target was assumed to be a point mass with loading distributed evenly to the three fibers through rigid, zero mass elements. The results of the 
analysis are as follows: for a $5 \mathrm{~mm}$ long fiber length, the first two modes of bending occur along the cross-sectional axis ( $\mathrm{x}$ and $\mathrm{z}$ ) and have a frequency of $21.4 \mathrm{~Hz}$. The third mode is rotational and is in the $\mathrm{kHz}$ range, and is therefore most likely outside the range of amplification. For $4 \mathrm{~mm}$ long fibers, the frequency is $34.8 \mathrm{~Hz}$, and for the $3 \mathrm{~mm}$ long fibers, the frequency is $53.4 \mathrm{~Hz}$.

Based on experimental findings by Roger Gram at LLE, the target support was analyzed to determine the natural frequencies of the bending modes. The legs were $8 \mu \mathrm{m}$ in diameter and mm long, butt-mounted to the target (at the tips) on a $300 \mu \mathrm{m}$ diameter. The experiments indicate that the natural frequency of the target support is $52.5 \mathrm{~Hz}$. The experiments also showed that the natural frequencies of the first two bending modes can be increased by as much as a factor of five by tilting the fibers out at the bottom (making them non-parallel).

COSMOS was then used to compare to the experimental results. The target was assumed to be a point mass with loading distributed evenly to the three fibers through rigid, zero mass elements. Two cases were examined. One with the fibers mounted parallel. The second with the fibers tilted such that the fibers are on a $600 \mu \mathrm{m}$ diameter circle at the base $(300 \mu \mathrm{m}$ at the top). The findings indicate that for parallel fibers, the natural frequency is $135 \mathrm{~Hz}$, and for tilted fibers, the frequency is $242 \mathrm{~Hz}$. Furthermore, we find that for fibers which taper (from $8 \mu \mathrm{m}$ at the base to $1 \mu \mathrm{m}$ at the tip), their natural frequency is $53 \mathrm{~Hz}$ for both the parallel and tilted cases. The reason for the difference between experimental and finite element results is due to the modeling of the target as a point mass, and in modeling the point where the fiber touches the target as a clamped joint instead of a hinged joint.

For further information, please contact R. Mangano (GA).

\subsubsection{FILL STATION ACTIVITIES}

The design of the fill station is based upon the need for thick $(\sim 100 \mu \mathrm{m})$ liquid or solid layers of $D_{2}$ or DT fuel in large diameter $(1400 \mu \mathrm{m})$ targets. This requires that empty shells be filled to densities as high as $0.16 \mathrm{gm} / \mathrm{cc}$. Additionally, the pending Rochester site license limits the amount of tritium to one gram $(9600 \mathrm{Ci})$.

To achieve high density target fills, $D_{2}$ or DT gas (at room temperature) will be permeated through the shell wall. Pressures in excess of 1100 atm (at room temperature) are required for high density targets. Thin walled shells can only survive relatively small pressure 
differentials. Thus, the shells must be pressurized in small steps to prevent the shells from collapsing. With these guide lines, the conceptual design of the cryogenic fill station was developed. Engineering activities for FY93 included designing and demonstrating proof-ofprinciple of the high pressure (1500 atmosphere) booster system for the fill station.

It was determined that the most reliable method of achieving the required pressures, with a limited gas inventory, is to use a gas intensifier system. The intensifier is a mechanically driven piston using a stepper motor having 0.2 atmosphere increments. A Bourdon tube piezoelectric transducer $(20,000$ psia, $0.005 \%$ full scale accuracy) was used to measure pressure. LabView software was used to monitor the fill station during a fill cycle experiment.

The current $\mathrm{GA}_{2}$ fill station was used to supply gas at pressures up to 3500 psia. The gas intensifier is used to increase the pressure from 3500 psia to 20,000 psia, in one stroke. The high pressure branch is isolated from the existing system by valves, including a one-way check valve. All tubing, fittings, and valves in the high pressure section are rated to 60,000 psia. The fill station has been tested, compressing 3,000 psia He gas to 23,500 psia (1600 atmosphere), satisfying the FY93 milestone for 93UR01C, designing and demonstrating proof-of-principle of a high pressure booster system.

\section{For further information, please contact R. Mangano (GA)}

\subsubsection{CRYOGENIC TRANSPORT AND STORAGE}

The modular approach to the conceptual design of the OMEGA Upgrade Target Delivery System requires that the targets be moved cryogenically between modules. The design concept uses a cold transfer cryostat for this operation. Successful functioning of the cold transfer cryostat requires a vacuum seal that can be made and broken at cryogenic temperatures $(-20 \mathrm{~K})$ at least once. We have built an apparatus that allows us to test seals of various designs for their ability to be made and broken at cryogenic temperatures. We have had a number of seals of various designs made, as well as the associated test fixtures. They include gasket seals between both flat and blunt nose faces, and spring loaded C-rings in both radial and face configurations. Materials under consideration for gaskets include: Teflon, OFHC copper, stainless steel, silver-plated stainless steel, nickel, and silver-plated nickel. Materials under consideration for C-rings include: silver, aluminum, Teflon, Kel-F, polymerfilled Teflon, glass filled Teflon, and polyethylene. 
The cold transfer cryostat also requires a manipulator for picking up targets and racks of targets. The manipulator conceptual design, from the conceptual design report, enveloped the target in a cavity at the bottom of the cryostat cold finger and then clamped onto the target's base. This design requires a high degree of precise alignment of the cavity to the target to prevent the target from being destroyed during the retrieval process. We produced a new manipulator design concept that requires less precision alignment and offers fewer opportunities for target damage. The new concept has a manipulator mounted on a small arm at the bottom of the cryostat cold finger. This is lowered past the target to the target base level, while well away from the target. The entire cryostat is then moved horizontally until the manipulator encounters the target base. It is the motion of the cryostat as a whole that induces any required motion in the manipulator. This obviates the need for a cryogenic $x-y$ translation stage at the bottom of the storage/transport dewar. With a mechanical actuator no longer running through the entire length of the cold transfer cryostat, its design has also been simplified. Translation stages and a bellows stage are added to the top of modules using the cold transfer cryostat, to give the cryostat its required motion. Included in the original conceptual design is the design for the storage/transport dewar system.

Several prototype target manipulators were built and operated in a test stand. The stand consisted of a 3/4 meter long rod connected to a three dimensional translation stage mounted on a platform. A keyhole manipulator is shown picking up a target in Fig. 7-3. Because of its gentleness in operation and omni-directional pickup capability, we have found the keyhole design to be the preferred manipulator design.

For further information, please contact $\operatorname{Dr} . N$. Alexander (GA)

\subsubsection{PROGRAM PLANNING}

As part of the engineering task, a project management plan [Ref. 7-4] was delivered to the University of Rochester. The document presented the Management Plan for the Cryogenic Target Delivery System. This plan presented the basic Work Breakdown Structure, Management Structure, Project Work Plan, Cost and Manpower, Schedule and Project Management Procedures.

For further information, please contact Dr. R. L. Fagaly (GA). 


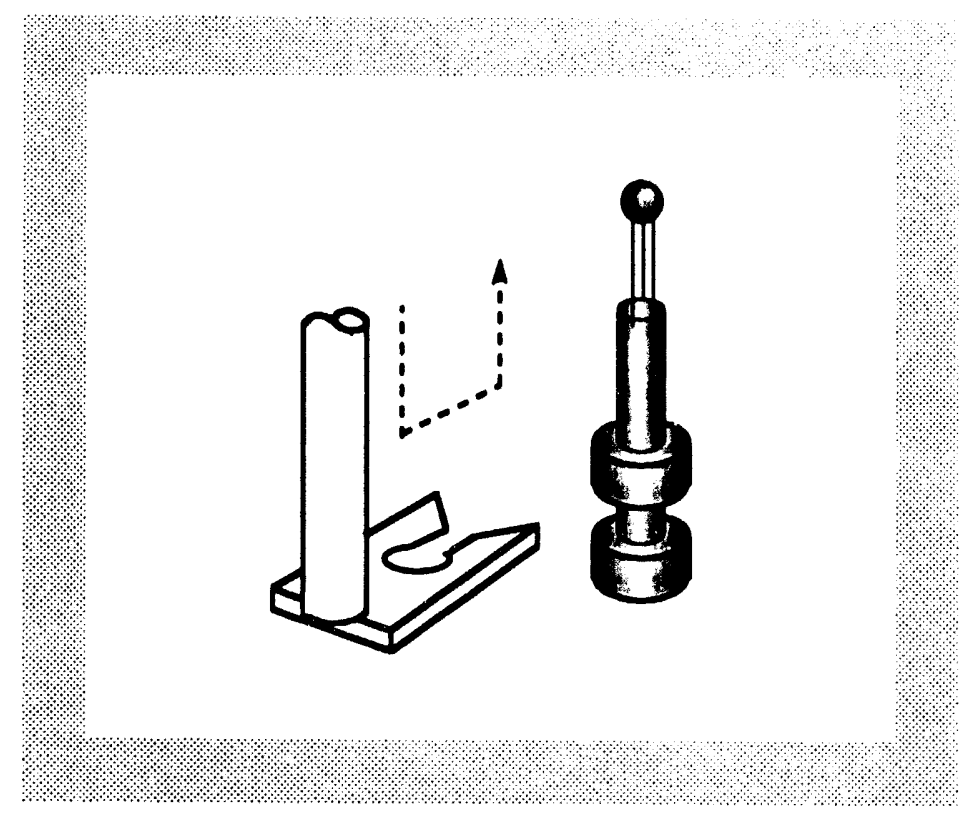

Fig. 7-3. The keyhole manipulator used for picking up targots.

\subsection{REFERENCES}

[7-1] Fagaly, R.L., and Bittner, D.N., "Design Requirements Report: University of Rochester Cryogenic Target Delivery System," General Atomics Report GA-C20937 (1992).

[7-2] Fagaly, R.L., Alexander, N.B., Bittner, D.N., Bourque, R.F., Dahms, C., Hendricks, C., Lindgren, J., and Miller, W.J., "Conceptual Design Report for the University of Rochester Cryogenic Target Delivery System," General Atomics Report GA-C21093 (1992). See also, "Conceptual Design for the OMEGA Upgrade Cryogenic Target Delivery System," Proceedings of the IEEE/NPSS Symposium on Fusion Engineering (1994) to be published.

[7-3] Varadarajan, V., Kim, K., and Bernat, T., J. Vac. Sci. Technol. A 5, (1987) 2750.

[7-4] Project Management Plan for the OMEGA Upgrade Cryogenic Target Delivery System, unpublished (1993). 
SECTION 8

\section{LLE MATERIALS DEVELOPMENT}




\section{LLE MATERIALS DEVELOPMENT}

In FY93, we developed new ICF capsule materials for use by UR/LLE.

\subsection{INTRODUCTION}

The LLE Materials Development task had two components: synthesis of poly[perdeutero(trimethylsilyl)styrene], and synthesis of a polymer which is opaque at $350 \mathrm{~nm}$ and transparent at visible wavelengths. The deuterated silylstyrene polymer is used in ICF capsules as a neutron diagnostic. The UV-opaque polymer is used to enhance the efficiency of direct-drive capsule implosions and has optical requirements which anthracene satisfies, so the task was to put anthracene into a polymer form.

\subsection{POLY[PERDEUTERO(TRIMETHYLSILYL)STYRENE]}

The synthesis of this material requires the synthesis of the monomer trimethylsilylstyrene, which is typically made from the Grignard of chlorostyrene and trimethylsilylstyrene. However, in this deuterated version, this route is problematic since the deuterated version of chlorostyrene is not commercially available. Cambridge Isotope was approached for a custom synthesis, but they required 3-4 months on a best effort basis. Since LLE required a more rapid delivery, we hought commercially available perdeuterostyrene, and sought to convert it to perdeuterobromostyrene. This synthesis required the following sequence:

(1) Conversion of $\mathrm{C}_{8} \mathrm{D}_{8}$ (perdeuterostyrene) to perdeutero-2-phenylethanol, by a hydroboration reaction of the styrene with $\mathrm{NaBD}_{4}$ and $\mathrm{BF}_{3}$-etherate, followed by hydrogen peroxide [Ref. 8-1]. This reaction seemed to proceed with near $100 \%$ yield.

(2) Bromination on the phenyl ring of 2-phenylethanol, using $\mathrm{Br}_{2}$ with $\mathrm{Fe}$, to make the 4-bromophenyl-2-ethanol [Ref. 8-2]. We anticipated a yield of near $100 \%$. 
(3) Dehydration of the 4-bromo-2-phenylethanol to form 4-bromostyrene [Ref. 8-3]. This reaction appeared to be the problem step, since several literature references all called for dripping the material onto $\mathrm{KHSO} 4$ at $220^{\circ} \mathrm{C}$, with distillation of water and the product into a receiving flask. This reaction is a non-standard reaction for dehydration to form an alkene, and would appear to risk thermal polymerization of the resulting styrene. The literature references claim some $50 \%$ yield of product. The key step (3) was done on non-deuterated material, and the desired product was found in the receiving flask.

Synthesis of perdeuterotrimethylsilylchloride was done in two steps:

(4) Phenyltrichlorosilane was reacted with $\mathrm{CD}_{3} \mathrm{MgCl}$, available commercially, to form phenyltrimethylsilane.

(5) The phenyl ring was removed with gaseous $\mathrm{HCl}$, to form the deuterated trimethylsilylchloride plus benzene.

Thus, we proceeded with the reactions, starting from 200 grams of $C_{8} D_{8}$, which was the estimated quantity required to obtain 50 grams of resulting polymer. In reaction (2), we had a resulting dark material, which we originally presumed was excess $\mathrm{Br}_{2}$ or some iron alcoxide impurity. However, after attempting to destroy the $\mathrm{Br}_{2}$ with thiosulfate, and distillation of the product, we had a large ( $>50 \%$ ) yield of black tar. The distillate had the desired bromophenylethanol, as well as other impurities (ethylbenzene, unreacted $C_{8} D_{8}$, and some dibromo-materials). Actually, the bromophenylethanol consisted of all six isomers, combinations of $\mathrm{Br}$ on three positions on the ring, times the two positions of the $\mathrm{OH}$ of ethanol. However, the low yield of $40 \%-50 \%$ was problematic. We suspect that the tar resulted from the DBr evolved from the bromination, which dehydrated the alcohol to styrene, and which then polymerized to form polystyrene-like tar.

When we performed reaction (3) with the deuterated bromophenylethanol, we did not obtain the desired product bromostyrene. However, in the distillate was some 4-bromophenylethylbromide, as determined by Gas Chromatography (GC) and Mass Spectrometry (MS). This material had been one of the impurities in the previous reaction mixture. We reacted this material with potassium isopropoxide to form 4-brom sstyrene [Ref. 8-4]. Reacting this material with $\mathrm{Mg}$, followed by perdeutero(trimethylsilylchloride), resulted in some 10-20 grams of perdeutero(trimethylsilyl)styrene, much less than the 100 grams of monomer we needed. In FY94, we recommend having perdeuterochlorostyrene custom made, so we can 
make a larger quantity of monomer. The existing monomer will be added to form the desired 50 grams of poly[perdeutero(trimethylsilyl)styrene].

\subsection{UV-ABSORBER MATERIALS}

The task was to put anthracene into a polymer form. The simplest two ways to make these two polymers are to replace the phenyl ring in styrene with anthracene to make a polymer akin to polystyrene [called poly(vinylanthracene)], and to replace the phenyl rings in parylene with anthracene to make $(2,2)(9,10)$ anthracenophane. The anthracenophane material thus produced ought to make a vapor depositable polymer, akin to the polymer made from parylene. The literature shows that the vapor deposited polymer is identical in structure to the poly(vinylanthracene) polymer [Ref. 8-5]. Thus, the two materials give the same ultimate material, but allow for different processing. The polyvinylanthracene polymer made from vinylanthracene is akin to polystyrene, and can be used in making shells via drop tower or microencapsulation techniques, while the anthracenophane material makes a vapor depositable coating akin to parylene.

\subsubsection{POLY(VINYLANTHRACENE)}

We had originally anticipated poly(vinylanthracene) would polymerize like styrene, across the vinyl units, leaving pendent anthracenes. However, the literature shows that the polymer actually polymerizes from the vinyl group across the 9,10 position of the anthracene, so that the anthracene is in the polymer backbone [Ref. 8-5].

We obtained 9-vinylanthracene from Aldrich, a chemical supply company. The literature states that polymerization is difficult [Ref. 8-6]. One route uses benzoyl peroxide and thermal heating at $120^{\circ} \mathrm{C}$ for 20 days. A more rapid route uses $\mathrm{SnCl}_{4}$. We made batches by both methods. The thermal heating route resulted in a red brick, which was broken up, dissolved in benzene, and reprecipitated to give a yellow polymer. The tin tetrachloride reaction is faster, and the polymer is precipitated by pouring into isopropanol. $\mathrm{The}_{\mathrm{SnCl}}$ route resulted in some $40 \%$ yield of yellow polymer.

We performed a couple of simple microencapsulation runs, to show the polymer could make shells. However, upon drying, many of the shells cracked, probably due to their low molecular weight ( $<8,000$ according to the literature). For its application to composite ICF 
capsules, there is little need for UV-absorption in the inner-most layer, so this line of investigation was not pursued further.

The UV-visible spectrum of poly(vinylanthracene), shown in Fig. 8-1, was obtained from solutions of the polymer. A solution route was chosen, to give a uniform path length of $1 \mathrm{~cm}$, and a well defined dilute concentration. From the solution concentration, one can calculate the

\section{UV-Visable Spectrum of Poly(vinylanthracene)}
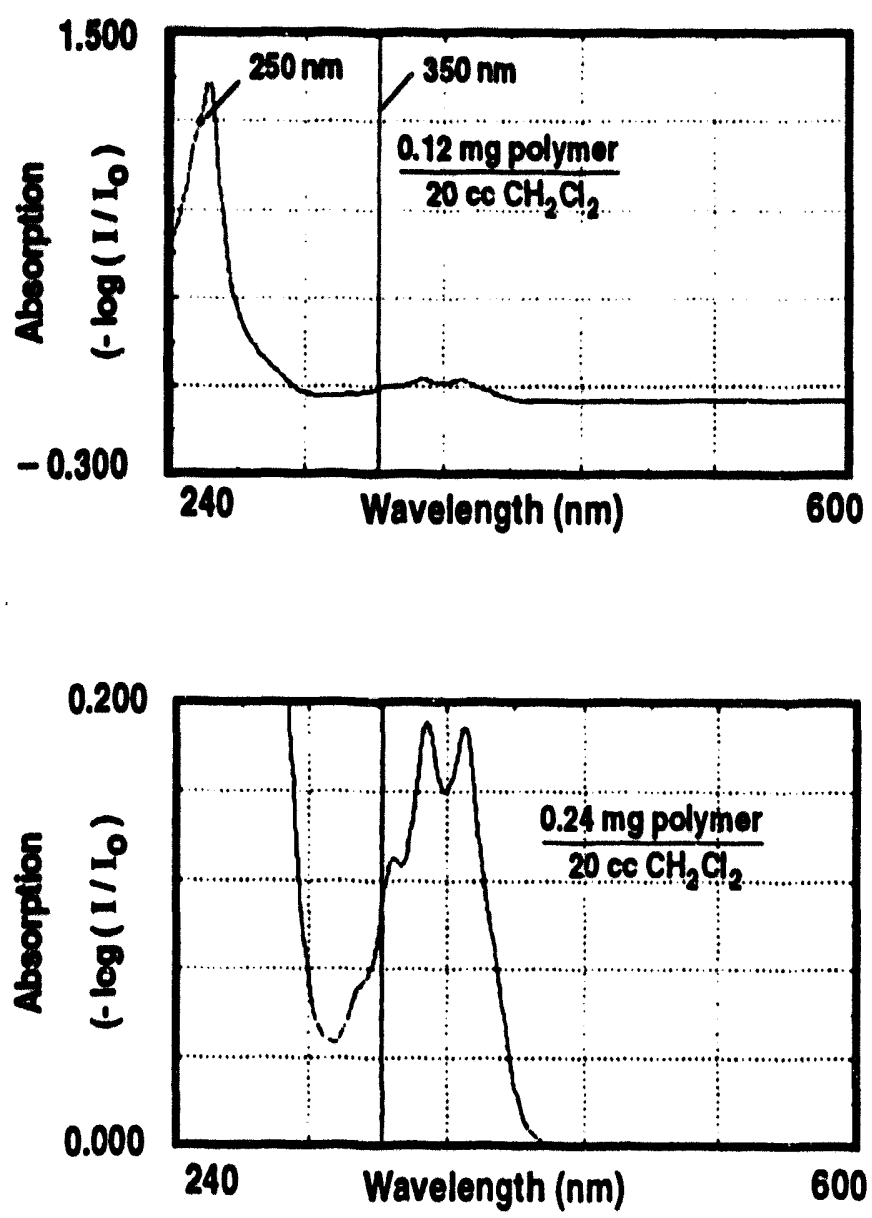

Fig. 8-1. UV-visable spectrum of poly(vinylanthraceno). 
"molar" absorption coefficient, and by estimating the density of the polymer as 1.0, calculate the transmission of $350 \mathrm{~nm}$ light through $10 \mu \mathrm{m}$ of polymer as $3 \times 10^{-9}$. This transmission is comparable to the transmission LLE achieves by depositing several hundred angstroms of aluminum onto their shells to make them opaque to UV. By contrast, the polymer is very transparent above $420 \mathrm{~nm}$. This polymer has the desired optical properties.

Table 8-1 gives the UV-absorption characteristics of poly(vinylanthracene) for various capsule coating thicknesses at wavelengths of interest to ICF experimentalists.

TABLE 8-1

\begin{tabular}{c|c|c}
\hline \multicolumn{3}{|c}{ UV-Absorption of Poly(vinylanthracene) } \\
\hline $\begin{array}{c}\text { Wall Thickness } \\
(\mu \mathrm{m})\end{array}$ & $\begin{array}{c}\text { Absorption at } 350 \mathrm{~nm} \\
\text { (Abs. = Log (I/ Io) }\end{array}$ & $\begin{array}{c}\text { Absorption at 250 nm } \\
\text { (Abs. = Log (I/ Io) }\end{array}$ \\
1 & -0.86 & -17.6 \\
5 & -4.28 & -88.1 \\
10 & -8.56 & -176.2 \\
\hline
\end{tabular}

\subsubsection{ANTHRACENOPHANE}

The synthesis of $(2,2)(9,10)$ anthracenophane $\mathrm{C}_{32} \mathrm{H}_{24}$ was done by Golden's method [Ref. 8-7]. Anthracene is reacted with paraformaldehyde and $\mathrm{HCl}$ to make the $(9,10)$ dichloromethylanthracene. A Soxhlet extraction of the dichloromethylanthracene with acetone brings small amounts of the material into a pot with $\mathrm{NaI}$, which forms $\mathrm{I}_{2}, \mathrm{NaCl}$, and the anthracenophane. This extraction synthesis is slow, making about 5 grams of material per day. The yellow-orange anthracenophane was filtered and washed to remove the other products. NMR measurements showed a very pure material (> 95\% purity). GC and MS measurements showed the anthracenophane was cleaved in the column, to form the polymerizable $\mathrm{C}_{16} \mathrm{H}_{12}$. 
We performed depositions of the anthracenophane in a tube furnace. The material sublimed at $150^{\circ}-200^{\circ} \mathrm{C}$ in a nitrogen flow, and the dimer was cleaved at $250^{\circ}-300^{\circ} \mathrm{C}$. The hot material was passed onto glass plates near room temperature. Scanning electron microscope examination showed a smooth non-crystalline deposition surface. We did not pursue optimization of the deposition process.

For additional information, please contact Dr. B. McQuillan (GA).

\subsection{REFERENCES}

[8-1] Pavia, D.L., Lampman, G.M., and Kriz, G.S., "Introduction to Organic Laboratory Techniques: A Contemporary Approach," 2nd edition, Sauners College Publishing, New York, (1982) Chapter 30.

[8-2] Modeled on the bromination of o-xylene: Wisansky, W.A., and Ansbacher, S., "Organic Syntheses," Collective Vol. 3, (1948) 138.

[8-3] Overberger, C.G., and Saunders, J.H., "Organic Syntheses," Collective Vol. 3, (1948) 204.

[8-4] Saunders, W.H., and Edison, D.H., J. Amer. Chem. Soc., 79, (1956) 3712.

[8-5] Michel, R.H., J. Polymer Science, Part A 2, (1964) 2533.

[8-6] Bergmann, E.D., and Katz, D., J. Chem. Soc., 3216, 1958; Stolka, M., Yanus, J.F., and Pearson, J.M., Macromolecules, 9(5), (1976) 715.

(8-7] Golden, J.H., J. Chem. Soc., (1961) 3741. 
SECTION 9

FY93 PUBLICATIONS 


\section{FY93 PUBLICATIONS}

Anikney, J.S., "Relating Defects in Plasma-Deposited Coatings to Irregularities on Spherical Substrates," in the Proceedings of the Ninth Target Fabrication Specialists' Meeting, Monterey, California, July 6-8, 1993.

Hoppe, M.L., Stephens, R.B., and Steinman, D.A., "PVA Layer Characterization," in the Proceedings of the Ninth Target Fabrication Specialists' Meeting, Monterey, California, July 6-8, 1993.

Steinman, D.A., Stephens, R.B., and Hoppe, M.L., "ICF Target Characterization Using Index Matching Fluids," in the Proceedings of the Ninth Target Fabrication Specialists' Meeting, Monterey, Califomia, July 6-8, 1993.

Stephens, R.B., "Computerization of Characterization," in the Proceedings of the Ninth Target Fabrication Specialists' Meeting, Monterey, California, July 6-8, 1993.

Schultz, K.R., "Capabilities of the ICF Target Fabrication Support Contractor," in the Proceedings of the Ninth Target Fabrication Specialists' Meeting, Monterey, California, July 6-8, 1993.

Monsler, M.J., "A Simple Model of the Thermal Layering of Cryogenic Deuterium Fuel in an ICF Capsule," in the Proceedings of the Ninth Target Fabrication Specialists' Meeting, Monterey, California, July 6-8, 1993.

Sater, J.D., "Micro-Calorimetry as a Method to Measure Fuel Mass in ICF Capsules," in the Proceedings of the Ninth Target Fabrication Specialists' Meeting, Monterey, California, July 6-8, 1993.

Nelson, D., Lilley, E., Cheung, L., and Soane, D., "Production of Polymer Shells by Controlled-Mass Microencapsulation," in the Proceedings of the Ninth Target Fabrication Specialists' Meeting, Monterey, California, July 6-8, 1993. 
Nelson, D., Cheung, L., Lim, F., and Soane, D., "Modeling of Microencapsulation," in the Proceedings of the Ninth Target Fabrication Specialists' Meeting, Monterey, California, July 6-8, 1993.

Schroen-Carey, D., "An Experimental Design Approach to Microencapsulation," in the Proceedings of the Ninth Target Fabrication Specialists' Meeting, Monterey, California, July 6-8, 1993.

Denton, A., and Lindsey, E., "SEM Analysis of Target Contamination Caused by Reaction Tube Etching," in the Proceedings of the Ninth Target Fabrication Specialists' Meeting, Monterey, California, July 6-8, 1993.

Lilley, E., Nelson, D., Cheung, L., and Soane, D., "Production of Microencapsulated Polymer Shells with the Triple-Orifice Controlled-Mass System," in the Proceedings of the Ninth Target Fabrication Specialists' Meeting, Monterey, California, July 6-8, 1993.

Alberts, T.E., and Kaae, J.L., "Free-Standing Foil Fabrication," in the Proceedings of the Ninth Target Fabrication Specialists' Meeting, Monterey, California, July 6-8, 1993.

Brown, L.C., Husband, D.O., and Baugh, W.A.,"Hot Box Design for a Barrier Layer Coating Tower," in the Proceedings of the Ninth Target Fabrication Specialists' Meeting, Monterey, California, July 6-8, 1993.

McQuillan, B.W., "Synthesis and Characterization of $350 \mathrm{~nm}$ UV Absorbing Barrier Materials," in the Proceedings of the Ninth Target Fabrication Specialists' Meeting, Monterey, California, July 6-8, 1993.

Parks, P.B., and Fagaly, R.L., "Layering Targets by Microgravity," in the Proceedings of the Ninth Target Fabrication Specialists' Meeting, Monterey, California, July 6-8, 1993.

Schneir, I., and McQuillan, B.W., "Production of Large Polystyrene Shells," in the Proceedings of the Ninth Target Fabrication Specialists' Meeting, Monterey, California, July 6-8, 1993.

Stephens, R.B., and Wittman, R., "Simplified Fringe Analysis for Wall Thickness Measurements," in the Proceedings of the Ninth Target Fabrication Specialists' Meeting, Monterey, Califomia, July 6-8, 1993. 
Bittner, D.N., "Surface Characterization by Interferometry," in the Proceedings of the Ninth Target Fabrication Specialists' Meeting, Monterey, California, July 6-8, 1993.

Beighley, D.H., and Steinman, D.A., "Range Extension of Interference Wall Thickness Measurements," in the Proceedings of the Ninth Target Fabrication Specialists' Meeting, Monterey, California, July 6-8, 1993.

Stephens, R.B., and Hoppe, M.L., "Rapid Shell Evaluation by Rotating Image Interference Fringe Analysis," in the Proceedings of the Ninth Target Fabrication Specialists' Meeting, Monterey, California, July 6-8, 1993.

Stephens, R., Wittman, R., "Inertial Fusion Shell Wall Thickness from Interference Fringe Shape Analysis," presented at the American Vacuum Society, November 11-15, 1993, Orlando, Florida.

Schultz, K.R., "ICF Target Fabrication and Technology Development Support," General Atomics Report GA-C20555.

Fagaly, R., et al, "Conceptual design report for the University of Rochester cryogenic target delivery system," General Atomics Report GA-A21093.

Stephens, R., "Fast-formed liquid surfaces for inertial confinement fusion target shells," IEEE/NPSS meeting, Hyannis, Massachusetts, October 1993. 

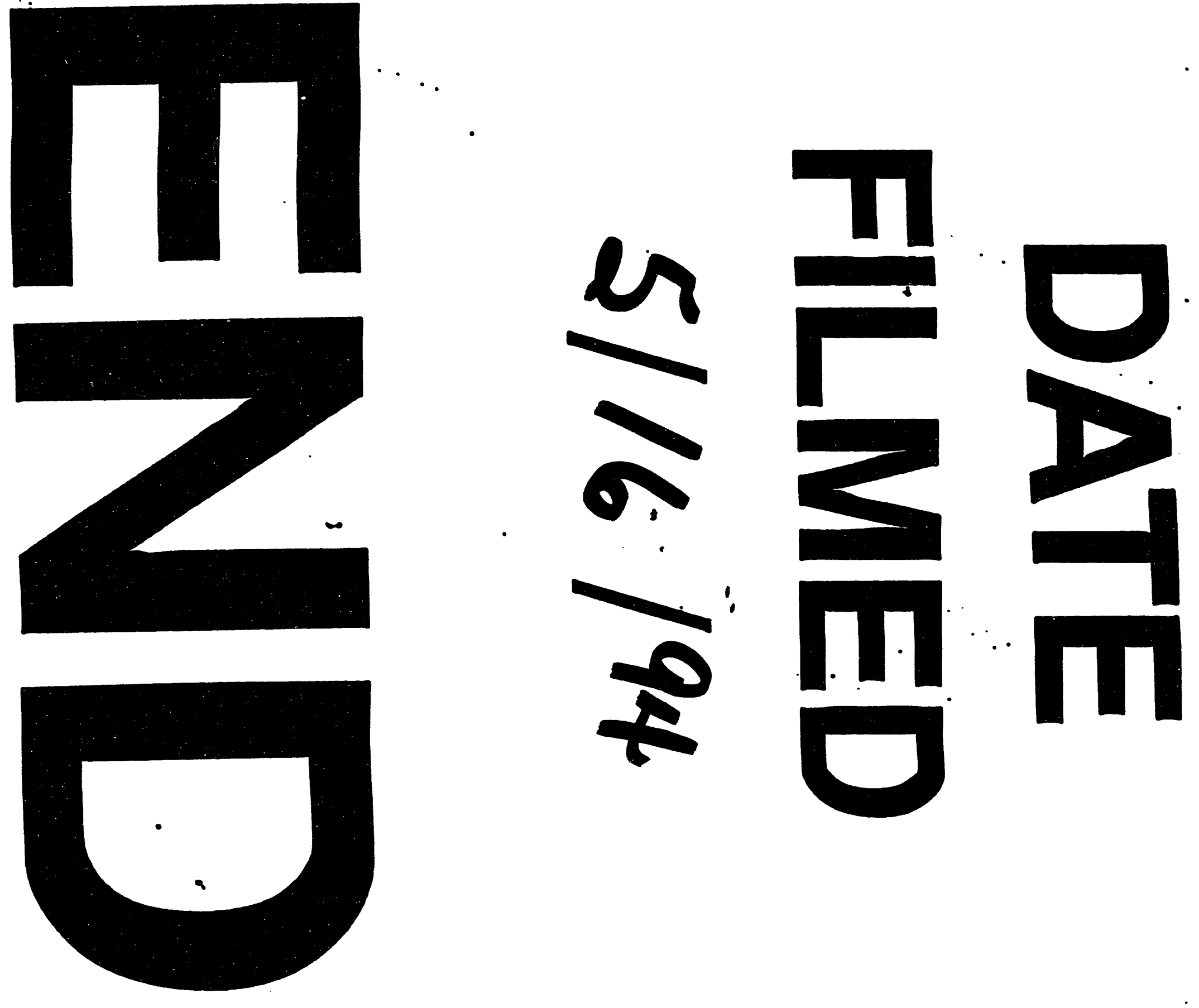
